\begin{abstract}
Title of Document:

STRUCTURAL RELIABILITY OF NOVEL 3-D INTEGRATED THERMAL PACKAGING FOR POWER ELECTRONICS.

Sumeer Khanna, M.S., 2015

Directed By:

Professor, F. Patrick McCluskey, Department of Mechanical Engineering

Thermal management has become increasingly important to ensuring the reliability of power electronics components due to the continuing increase of device power and integration levels. New approaches to provide the necessary thermal management include the development of embedded two-phase cooling systems. However, the reliability of such devices and that of their integration into the power electronics package have yet to be studied. This thesis details a Physics of Failure (PoF) based structural reliability analysis of novel 3-D integrated thermal packaging for next generation Power Electronics. The cooling technology aims to combine two-phase embedded manifold microchannel cooling in thin film evaporation mode with thermoelectric hot-spot cooling using a high conductivity Mini-contact. This study will focus on thermo-mechanical stress analysis of three different Mini-contact structures, micro-fin structure and reliability prediction of solder joint at various
\end{abstract}


levels in Power Electronics package based on Engelmaier's failure model for SAC 305. 


\title{
STRUCTURAL RELIABILITY OF NOVEL 3-D INTEGRATED THERMAL PACKAGING FOR POWER ELECTRONICS
}

\author{
By
}

Sumeer Khanna

Thesis submitted to the Faculty of the Graduate School of the University of Maryland, College Park, in partial fulfillment of the requirements for the degree of

Master of Science

2015

Advisory Committee:

Professor F. Patrick McCluskey, Chair

Professor Avram Bar-Cohen

Professor Michael Ohadi 
(C) Copyright by

Sumeer Khanna

2015 


\section{Acknowledgements}

It is my pleasure to thank everyone who helped me in the completion of my thesis work and for without their help, I would have never been successful.

It is an honor for me to thank first my advisor, Dr. F. Patrick McCluskey, whose mentorship has been invaluable in my graduate career and has taught me much about electronics packaging. His motivation, support and guidance will always be appreciated. I owe my deepest gratitude to my mentors Dr. Bar-Cohen, Dr. Ohadi and Dr. Bao Yang. Furthermore, I would like to thank DARPA and Dr. Martin for giving me a great platform to learn more on next generation Power Electronics. I am also obliged to Dr. Dasgupta for his assistance with structural modeling.

Special thanks go to Michael Manno and Raphael Mandel for their assistance whenever I asked them. Mike's vast knowledge and patience has taught me about thermal management and Finite Element modeling techniques. Raphael asked the hard questions and really pushed me to understand and explain. Additionally, I would like to thank Hannes Greve for sharing his knowledge and expertise with solder joint reliability. I'm grateful to many colleagues whose help and advice has made this thesis possible: Michael Fish, Ali Moeini, David Squiller, Caleb Serafy, Subramani Manoharan, Jennifa and many more.

I am deeply grateful to all my teachers at the University of Maryland, who have given their time and inspired me to love the field of engineering.

Last but not least, I would like to thank my parents and my siblings for their extended support and motivation. I dedicate this thesis to them. 


\section{Table of Contents}

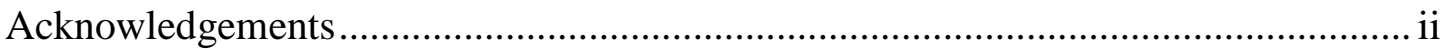

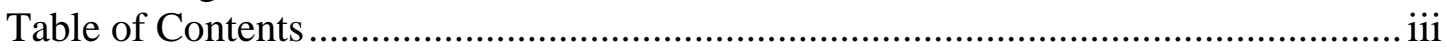

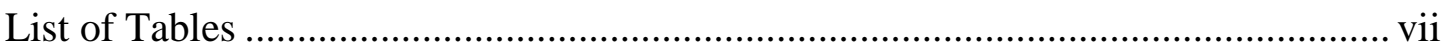

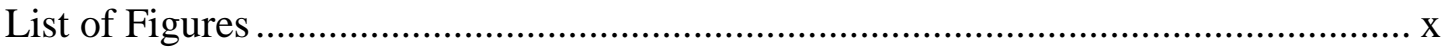

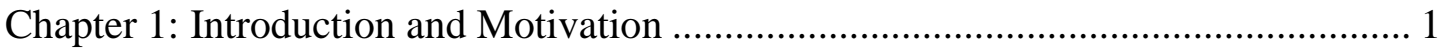

1.1 Power Electronics Materials: GaN on Si, GaN on SiC ..................................... 2

1.2 What is Thermally Integrated Power Electronics Packaging? ........................... 3

1.2.1 Design for Reliability ..................................................................... 4

1.2.2 Fundamentals of Thermal Management .................................................. 5

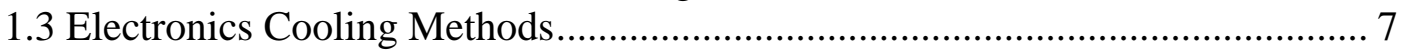

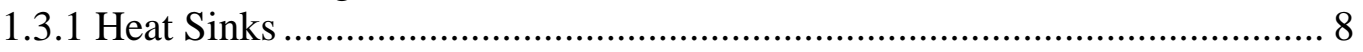

1.3.2 Thermoelectric Phenomena ..................................................................... 9

1.3.3 Hot Spot Cooling and related Reliability Issues .................................... 10

Chapter 2: Reliability Theory and Physics of Failure Models.................................. 13

2.1 Physics of Failure Perspective of Reliability .................................................. 13

2.2 Physics of Failure - POF process for Reliability Assessment........................... 14

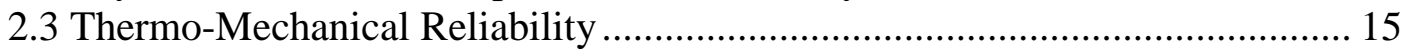

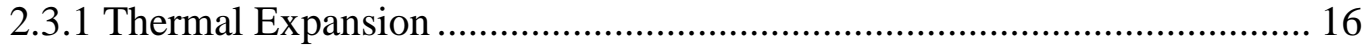

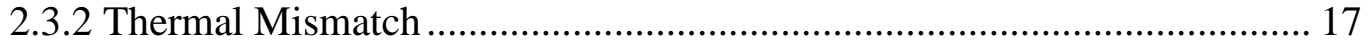

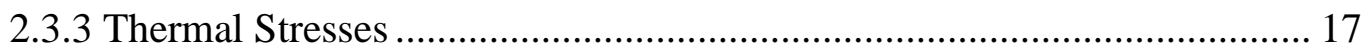

2.3.4 Stresses Generated due to Temperature Gradients .................................. 18

2.3.5 Thermo-mechanical Failure Mechanisms .................................................. 19

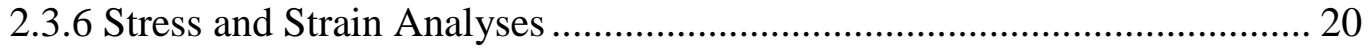

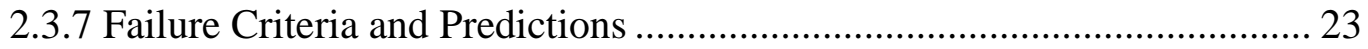

2.4 Physics of Failure - PoF based Reliability Prediction Models ....................... 24

2.4.1 Weibull Statistical Distribution........................................................... 24

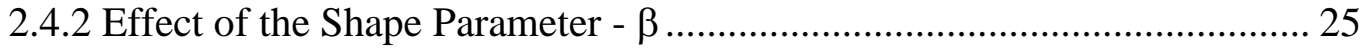

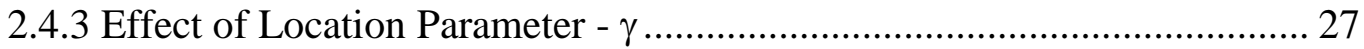

2.5 Fatigue Life Prediction Physics of Failure - PoF based Reliability Models ..... 28

2.5.1 Coffin Manson Model.............................................................................. 28

2.5.2 Relationship for Coffin-Manson Equation............................................. 29

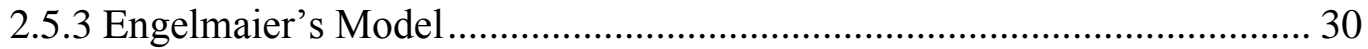

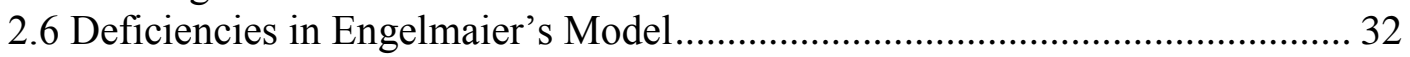

2.6.1 Shortcomings in the Fatigue Model ................................................... 32

2.6.2 Solder Joint Height and Quality ......................................................... 33

2.6.3 Local Coefficient of Thermal Expansion - CTE Mismatches .................. 33

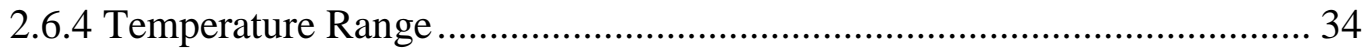

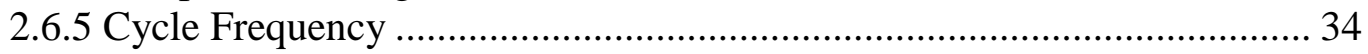

2.7 Engelmaier's Model for Lead Free Solders ............................................... 34

2.8 Yielding Phenomenon for Ductile and Brittle Materials ................................ 36

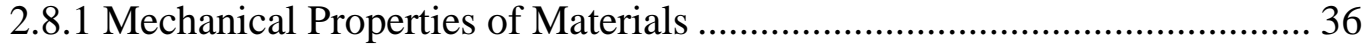

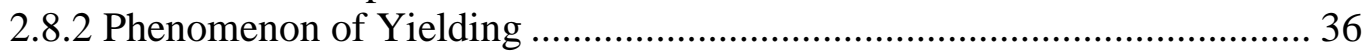

2.8.3 Cauchy Stress Tensor......................................................................... 37 


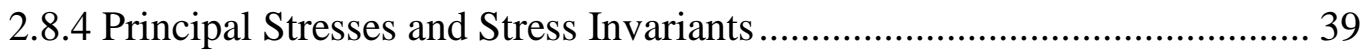

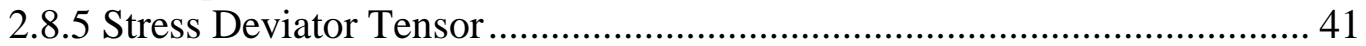

2.8.6 Von Mises Yield Criterion..................................................................... 42

2.8.7 Maximum Principal Stress Theory …………………………………..... 44

Chapter 3: TE Cooler Integration and Material Selection ......................................... 46

3.1 Thermoelectric Mini-contact Based Cooling for Hotspot Heat Flux Removal 46

3.1.1 Mini-contact based Cooling Methodology ................................................. 47

3.1.2 Integration Options .............................................................................. 48

3.1.3 Material Selection ............................................................................... 54

3.1.4 Silver-Diamond Composite as Thermal Management Material ................. 56

3.1.5 Rule of Mixtures for C.T.E. Prediction.................................................... 57

3.1.6 Other Models for C.T.E. Prediction .......................................................... 59

3.1.7 Prediction of Thermal Conductivity using FE Analysis ............................... 60

3.1.8 Functionally Graded Silver-Diamond Composite ……………………......... 64

Chapter 4: Finite Element Model and Boundary Conditions........................................ 66

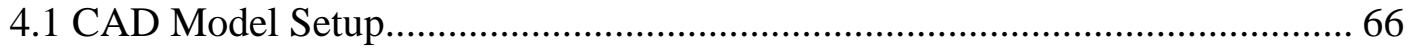

4.1.1 Thermo-electric Mini-contact based Cooling …………………………..... 67

4.1.2 Manifold-Microchannel Cooling ............................................................. 71

4.2 Material Properties.................................................................................. 72

4.2.1 Material Properties Data ....................................................................... 73

4.3 Element Selection and Mesh Generation ....................................................... 77

4.3.1 Mini-contact based Thermo-electric Cooling ............................................ 77

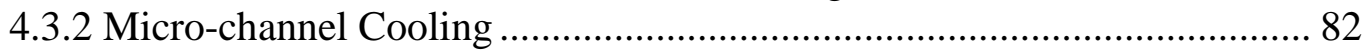

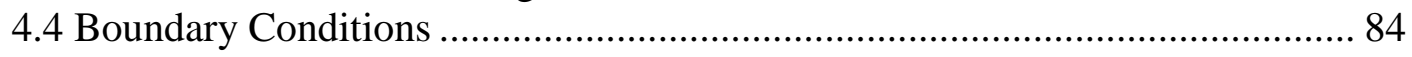

4.4.1 Mini-contact based Thermo-electric Cooling ............................................. 84

4.4.2 Manifold Microchannel Cooling.............................................................. 90

Chapter 5: Results: Thermo-mechanical Structural Analysis ....................................... 92

5.1 Integrated SiC Mini-contact based Thermoelectric Cooling ............................ 92

5.1.1 Temperature Contour Plot.................................................................... 92

5.1.2 Maximum Deformation Plot ................................................................. 93

5.1.3 Von-Mises Plastic Strain Plot .................................................................. 94

5.1.4 Critical Flaw Size - Bismuth Telluride Elements - $\mathrm{Bi}_{2} \mathrm{Te}_{3} \ldots \ldots \ldots \ldots \ldots \ldots \ldots \ldots . . . . .99$

5.1.5 Critical Flaw Size - Aluminum Nitride Headers....................................... 101

5.1.6 Critical Flaw Size - GaN on SiC ........................................................... 103

5.2 Discrete Mini-contact: Copper - T-shaped Model ............................................ 105

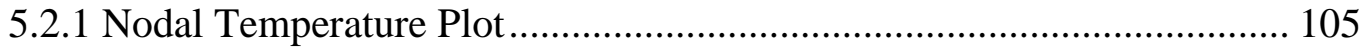

5.2.2 Displacement Contour Plot ..................................................................... 105

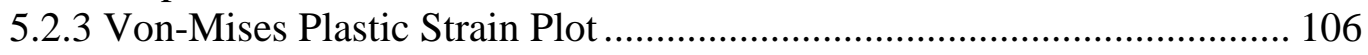

5.2.4 Critical Flaw Size - Bismuth Telluride Elements .................................... 109

5.2.5 Critical Flaw Size - AlN Headers ............................................................ 111

5.2.6 Critical Flaw Size - GaN on SiC Device …………………………........ 112

5.3 Discrete Mini-contact: Copper - Lofted Model ……………………............... 114

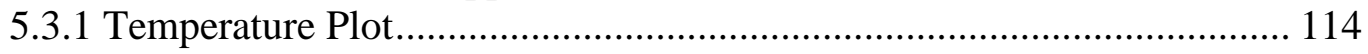

5.3.2 Displacement Contour Plot .................................................................... 114

5.3.3 Von-Mises Plastic Strain Plot ............................................................... 115

5.3.4 Critical Flaw Size - Thermo-electric Elements......................................... 118 
5.3.5 Critical Flaw Size - GaN on SiC substrate............................................... 121

5.4 Discrete Mini-contact: Copper - Taper Model................................................ 123

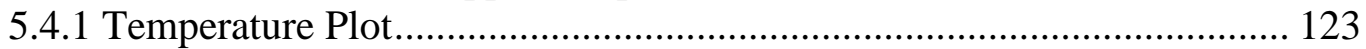

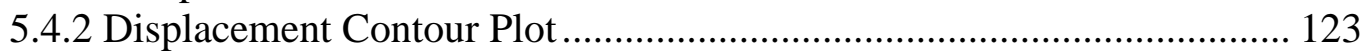

5.4.3 Von-Mises Plastic Strain Plot .............................................................. 124

5.4.4 Critical Flaw Size - Thermo-electric Elements and Headers.................... 127

5.4.5 Critical Flaw Size - GaN on SiC substrate............................................. 129

5.5 Discrete Mini-contact: Diamond - T-shaped Model...................................... 131

5.5.1 Nodal Temperature Plot ...................................................................... 131

5.5.2 Displacement Contour Plot ................................................................. 132

5.5.3 Design for Reliability: Mean Time to Failure - MTTF............................. 132

5.5.4 Maximum Principal Stress Plot - Diamond Mini-contact......................... 135

5.5.5 Critical Flaw Size - Thermo-electric Elements and Headers.................... 137

5.5.6 Critical Flaw Size - GaN on SiC substrate........................................... 139

5.6 Discrete Mini-contact: Diamond - Lofted Model ............................................ 141

5.6.1 Nodal Temperature Plot.................................................................... 141

5.6.2 Displacement Contour Plot .................................................................. 142

5.6.3 Design for Reliability: Mean Time to Failure - MTTF.............................. 143

5.6.4 Critical Flaw Size - Mini-contact and Thermo-electric Cooler ................ 145

5.6.5 Critical Flaw Size - GaN on SiC substrate............................................. 148

5.7 Discrete Mini-contact: Diamond - Taper Model ............................................ 150

5.7.1 Temperature Contour Plot................................................................. 150

5.7.2 Displacement Contour Plot ................................................................. 150

5.7.3 Design for Reliability: Mean Time to Failure - MTTF.............................. 151

5.7.4 Critical Flaw Size - Mini-contact and TE Cooler ..................................... 153

5.7.5 Critical Flaw Size - GaN on SiC substrate............................................ 157

5.8 Physics of Failure: Integrated SiC Mini-contact Model ................................ 159

5.8.1 C.T.E. Mismatch: SiC Mini-contact - AlN header .................................. 159

5.8.2 C.T.E. Mismatch: Thermo-electric Cooler …………………………...... 159

5.9 Physics of Failure: Discrete Copper Mini-contact Model ............................... 160

5.9.1 C.T.E. Mismatch: SiC - SAC305 - Copper...………………………....... 160

5.9.2 C.T.E. Mismatch: Copper - SAC305 - AlN.............................................. 160

5.10 Physics of Failure: Discrete Diamond Mini-contact Model ........................... 161

5.10.1 C.T.E. Mismatch: SiC - SAC305 - Diamond........................................... 161

5.10.2 C.T.E. Mismatch: Diamond - SAC305 - AlN ………………………........ 161

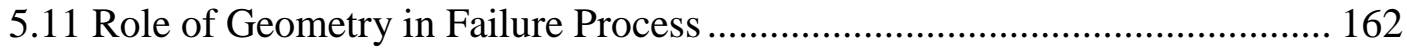

5.11.1 Geometry Effects on Reliability …………………………………....... 162

Chapter 6: Silver-Diamond Composite as Mini-contact Material ............................... 164

6.1 T-shaped Mini-contact: Silver-Diamond Composite........................................ 165

6.1.1 Temperature Contour Plot.................................................................... 165

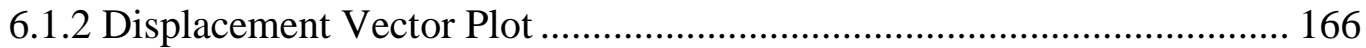

6.1.3 Thermo-electric Cooler Reliability …………………………………..... 166

6.1.4 Stress Distribution across Mini-contact .................................................. 167

6.1.5 Critical Flaw Size - Thermo-electric Headers and Elements.................... 168

6.2 Lofted Mini-contact: Silver-Diamond Composite …………............................. 169

6.2.1 Temperature Contour Plot...................................................................... 169 
6.2.2 Displacement Contour Plot

6.2.3 Thermo-electric Cooler Reliability ..................................................... 170

6.2.4 Stress Distribution across Mini-contact................................................. 171

6.2.5 Critical Flaw Size - Thermoelectric Elements and Headers .................... 172

6.3 Taper Mini-contact: Silver-Diamond Composite ........................................... 172

6.3.1 Temperature Contour Plot................................................................ 172

6.3.2 Displacement Contour Plot ................................................................. 173

6.3.3 Thermo-electric Cooler Reliability ..................................................... 173

6.3.4 Stress Distribution across Mini-contact structure .................................... 174

6.3.5 Critical Flaw Size - Thermoelectric Elements and Headers .................... 174

Chapter-7: Manifold Micro-channel Structural Reliability ...................................... 175

7.1 Manifold - Microchannel Two-Phase Cooling: Structural Modeling .............. 176

7.1.1 Temperature Contour Plot................................................................... 176

7.1.2 Critical Flaw Size - Micro-fin corner..................................................... 177

Chapter 8: Conclusions - Structural Reliability Modeling ...................................... 180

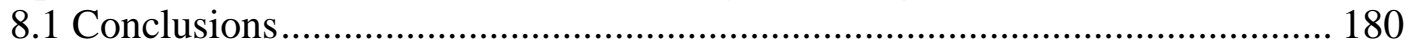

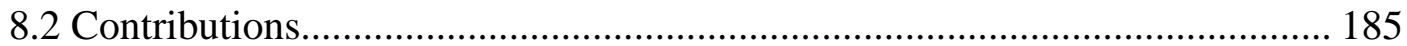

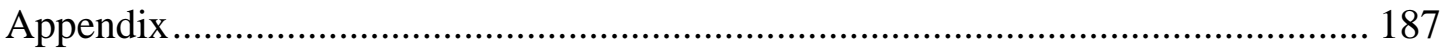

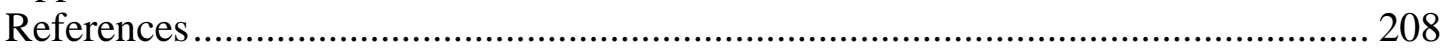




\section{List of Tables}

Table 2.1 Selection of Failure Theory Based on Type of Material............................24

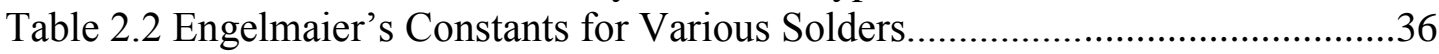

Table 3.1 Mechanical Properties of Diamond and Nano-sintered Silver.....................58

Table 3.2 Diamond Particle Spacing and Size Comparison.....................................63

Table 4.1 Material Properties Input to FEA Model................................................... 73

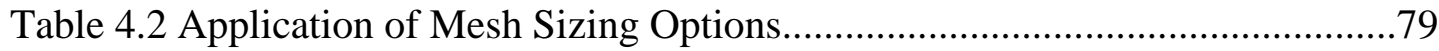

Table 4.3 Parameters set to the TE Cooler...................................................................86

Table 5.1 Reliability Predictions Solder Joint - Mini-contact/TE Cooler Header

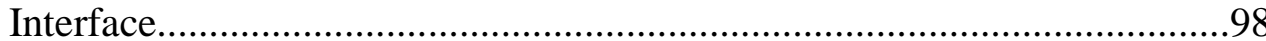

Table 5.2 Reliability Prediction, MTTF: Solder Joint - Copper Straps/Thermo-electric

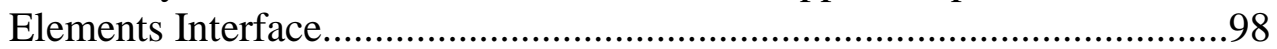

Table 5.3 Principal Stresses in Thermo-electric Element, Integrated SiC Mini-

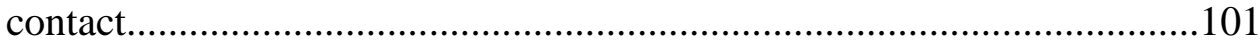

Table 5.4 Principal Stresses - TE Cooler Headers, Integrated SiC Mini-contact......102

Table 5.5 Principal Stresses - GaN on SiC substrate, Integrated SiC Minicontact

Table 5.6 Design for Reliability, MTTF: Chip Level Integration: SiC - Solder Joint -

Mini-contact, T-shaped $\mathrm{Cu}$ Mini-contact.

Table 5.7 Design for Reliability, MTTF: Solder Joint - Cooler/Contact Level Integration, T-shaped Cu Mini-contact.....................................................108

Table 5.8 Design for Reliability, MTTF Solder Joint - Copper Straps/Thermo-electric Elements Interface.

Table 5.9 Principal Stresses - Thermo-electric Elements, T-shaped Cu Minicontact.

Table 5.10 Principal Stresses - TE Cooler Header, T-shaped Cu Mini-contact........112

Table 5.11 Principal Stresses - GaN on SiC substrate, T-shaped Cu Minicontact.

Table 5.12 Design for Reliability, MTTF - Chip Level Integration, Lofted Cu Mini-

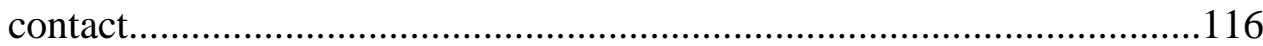

Table 5.13 Design for Reliability, MTTF - Contact/Cooler Integration....................117

Table 5.14 Design for Reliability, MTTF - Solder Joint inside TE Cooler................118

Table 5.15 Principal Stresses - Thermoelectric Elements, Lofted Cu Minicontact.

Table 5.16 Principal Stresses - Thermoelectric Cooler Headers, Lofted Cu Minicontact

Table 5.17 Principal Stresses - GaN on SiC, Lofted Cu Mini-contact......................122

Table 5.18 Design for Reliability, MTTF - Chip Level Integration.........................125

Table 5.19 Design for Reliability, MTTF - Mini-contact/Cooler Integration............125

Table 5.20 Design for Reliability, MTTF - Solder inside TE Cooler.........................126

Table 5.21 Principal Stresses - Thermoelectric Elements, Taper Cu Minicontact.

Table 5.22 Principal Stresses - TE Cooler Headers, Taper Cu Mini-contact............128

Table 5.23 Principal Stresses - GaN on SiC substrate, Taper Cu Mini-contact........129 
Table 5.24 Reliability Prediction, Solder Joint - Chip Level Integration Interface, Tshaped Diamond Mini-contact.

Table 5.25 Reliability Prediction, Solder Joint - Mini-contact/Thermoelectric Cooler Header Interface, T-shaped Diamond Mini-contact...............................134

Table 5.26 Reliability Prediction, MTTF - Solder Joint: TE Cooler.........................134

Table 5.27 Principal Stresses - Thermoelectric Elements, T-shaped Diamond Mini-

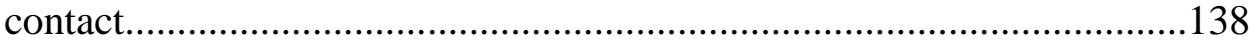

Table 5.28 Principal Stresses - Thermoelectric Cooler Headers, T-shaped Diamond

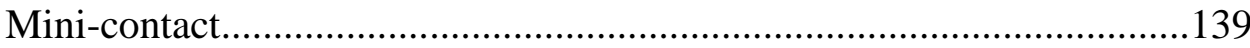

Table 5.29 Principal Stresses - GaN on SiC substrate, T-shaped Diamond Mini-

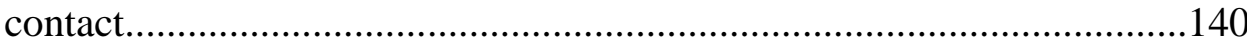

Table 5.30 Reliability Prediction, Solder Joint - SiC Substrate/Mini-contact Interface Lofted Diamond Mini-contact.

Table 5.31 Reliability Prediction, Solder Joint - Mini-contact/Thermoelectric Cooler Header Interface, Lofted Diamond Mini-contact..................................144

Table 5.32 Reliability Prediction, Solder Joint - TE Cooler, Lofted Diamond Mini-

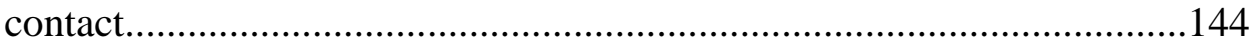

Table 5.33 Principal Stresses - Thermoelectric Elements, Lofted Diamond Mini-

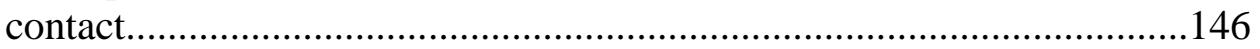

Table 5.34 Principal Stresses - Thermoelectric Headers, Lofted Diamond Mini-

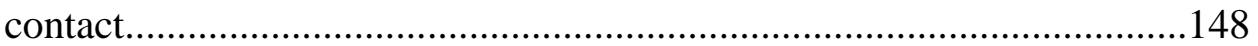

Table 5.35 Principal Stresses - GaN on SiC substrate, Lofted Diamond Minicontact.

Table 5.36 Reliability Prediction, Solder Joint - SiC Substrate/Mini-contact Interface, Taper Diamond Mini-contact.

Table 5.37 Reliability Prediction, Solder Joint - Mini-contact/Thermoelectric Cooler Header Interface, Taper Diamond Mini-contact.

Table 5.38 Reliability Prediction, Solder Joint - Copper Straps/Thermoelectric Cooler Elements Interface, Taper Diamond Mini-contact................................152

Table 5.39 Principal Stresses - Thermoelectric Elements, Taper Diamond Mini-

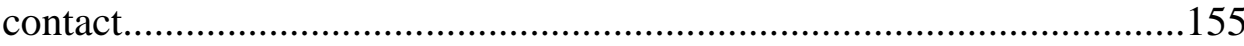

Table 5.40 Principal Stresses - GaN on SiC substrate, Taper Diamond Minicontact..................................................................................158

Table 6.1 60\% Diamond - 40\% Silver Composite Mechanical Properties.................165

Table 6.2 Reliability Prediction, MTTF: Solder Joint - Thermoelectric Cooler, Tshaped, Silver-Diamond Composite.

Table 6.3 Principal Stresses - Thermoelectric Header, T-shaped, Silver-Diamond Composite 168

Table 6.4 Principal Stresses - Thermoelectric Elements, T-shaped, Silver-Diamond

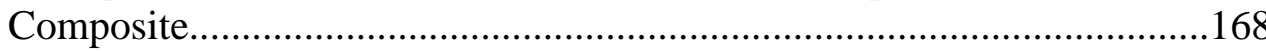

Table 6.5 Reliability Prediction, Solder Joint - Thermoelectric Cooler, Lofted, SilverDiamond Composite......................................................................171

Table 6.6 Principal Stresses - Thermoelectric Elements - Lofted, Silver - Diamond Composite 172

Table 6.7 Principal Stresses - Thermoelectric Headers - Lofted, Silver-Diamond Composite 
Table 6.8 Reliability Prediction, Solder Joint - Thermoelectric Cooler - Taper, Silver Diamond Composite

Table 6.9 Principal Stresses - Thermoelectric Headers - Taper, Silver-Diamond Composite.

Table 6.10 Principal Stresses - Thermoelectric Elements - Taper, Silver - Diamond

Composite. 174

Table 8.1 Reliability Comparison Integrated SiC Mini-contact and Discrete Copper Mini-contact.

Table 8.2 Reliability Comparison - Discrete Diamond Mini-contact........................181

Table 8.3 Reliability Comparison Silver-Diamond Composite as Mini-contact Material. 


\section{List of Figures}

Figure 1.1 Heat Removal through use of Heat Sink..............................................

Figure 1.2 Schematic Diagram of a Thermoelectric Cooler.........................................9

Figure 1.3 Explanation of Thermoelectric Phenomena.............................................9

Figure 1.4 Flip Chip Package with eTEC mounted on Heat Spreader.......................11

Figure 2.1 Effect of Cooling and Heating on Thermo-mechanical Stress

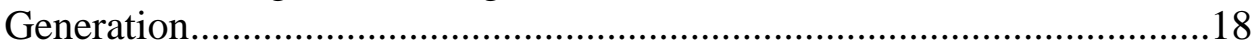

Figure 2.2 Schematic view of Flip Chip Ball Grid Array, FC PBGA.......................19

Figure 2.3 FEM Mesh Generation Example - 1um x 1um x 1um............................22

Figure 2.4 Effect of Shape Parameter on Weibull Failure Rate...............................26

Figure 2.5 Effect of location parameter on Weibull pdf........................................27

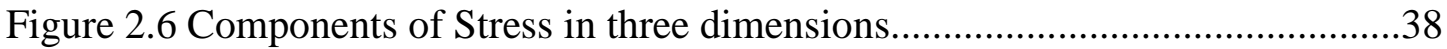

Figure 2.7 Von-Mises Yield Surface in Principal Stress Coordinates.........................43

Figure 2.8 Transformation to Principal Directions......................................................44

Figure 2.9 Yielding Phenomenon in Ductile and Brittle Materials...........................45

Figure 3.1 Schematic of TE Mini-contact and Conventional hot-spot cooling...........46

Figure 3.2 Integrated SiC Mini-contact Model - Quarter Symmetric.........................49

Figure 3.3 Approximate Package Dimensions - Side View.......................................50

Figure 3.4 Discrete T-shaped Mini-contact Quarter Symmetric Model.......................51

Figure 3.5 Lofted Mini-contact Quarter Symmetric Model......................................52

Figure 3.6 Side View - Lofted Mini-contact Model..............................................52

Figure 3.7 Taper Mini-contact Quarter Symmetric Model.........................................53

Figure 3.8 Side View - Taper Mini-contact Model...................................................54

Figure 3.9 Unit Model Representation of Particle - Matrix Phases.............................59

Figure 3.10 FE Model Setup for Prediction of Mechanical Properties........................61

Figure 3.11 Illustration of Application of Boundary Conditions

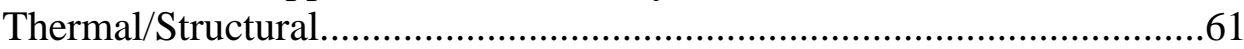

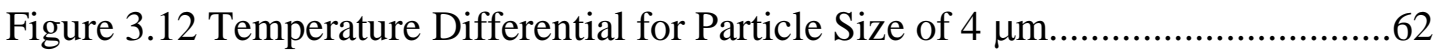

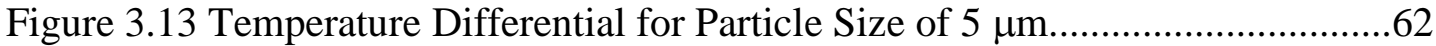

Figure 3.14 Illustration of Stress/Strain Plot at Diamond Particle - Silver Matrix

Interface for various Size and Spacing..............................................63

Figure 3.15 Functionally Graded Metal-Diamond Composite Concept......................64

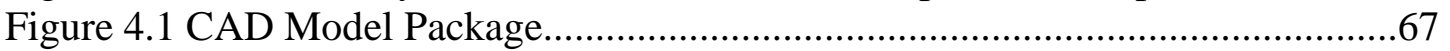

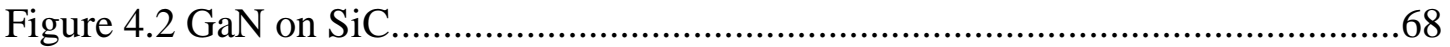

Figure 4.3 SiC die Quarter Symmetric Dimensions..............................................68

Figure 4.4 T-shaped, Lofted and Taper Mini-contact Dimensions.............................68

Figure 4.5 Figure 4.5 T-shaped Mini-contact, Quarter Symmetric CAD model.........69

Figure 4.6 Solder on Mini-contact for TE Cooler Integration..................................69

Figure 4.7 HV 37 Laird Thin Film Device............................................................ 70

Figure 4.8 Thermo-electric Cooler CAD model: HV 37 model................................70

Figure 4.9 Manifold-Microchannel Model - Forced Fed Cooling Technology............71

Figure 4.10 Unit Cell Model - Manifold Microchannel Cooling.................................71

Figure 4.11 Microchannel Base and Fin Dimensions..............................................72

Figure 4.12 Temperature Dependent Thermal Conductivity - Silicon Carbide............74 
Figure 4.13 True Stress vs True Strain for SAC 305 solder......................................74

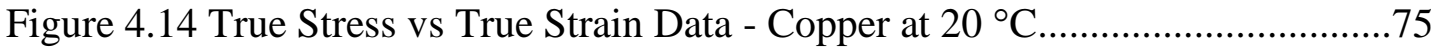

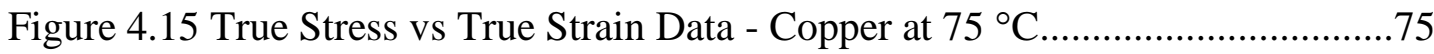

Figure 4.16 True Stress vs True Strain Data - Copper at $125^{\circ} \mathrm{C}$.................................76

Figure 4.17 True Stress vs True Strain - Copper at $175^{\circ} \mathrm{C}$.........................................76

Figure 4.18 Mesh Density Study: Hot-spot Temperature........................................79

Figure 4.19 Mesh Sizing across the Package ....................................................... 80

Figure 4.20 Hot-spot Region Element Sizing..................................................... 81

Figure 4.21 SiC Substrate Region - Mini-contact domain Sizing............................81

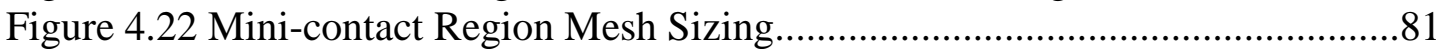

Figure 4.23 Solder Joint between TE Cooler and Mini-contact...................................82

Figure 4.24 TE Cooler Header Element Edge Length............................................82

Figure 4.25 Element Edge Length - TE Elements, Solder Joint and Copper Straps...82

Figure 4.26 Unit Cell Model - Microfluidics Cooling............................................83

Figure 4.27 CAD Model - Unit Cell Model - Microfluidics.......................................83

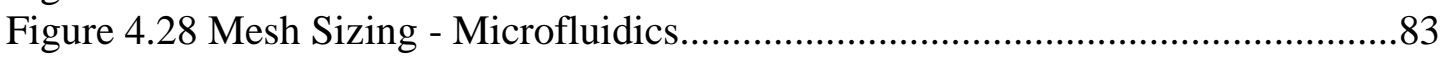

Figure 4.29 Application of Thermal Boundary Conditions.........................................84

Figure 4.30 Hot-spot Heat Flux of 5k W/cm ${ }^{2}$ - Quarter Symmetric Model................85

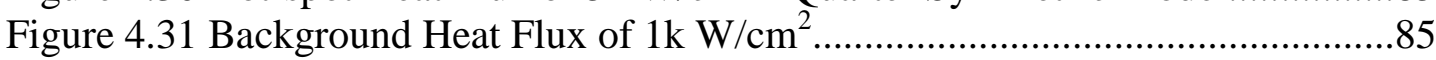

Figure 4.32 Application of Global Heat Transfer Convection Coefficient..................86

Figure 4.33 Flip Chip Package Assembly................................................................8

Figure 4.34 Package Constrained at bottom of GaN on SiC substrate........................84

Figure 4.35 Quarter Symmetric Boundary Condition on X face...............................89

Figure 4.36 Quarter Symmetric Boundary Condition on Y face................................89

Figure 4.37 Illustration of Thermal and Mechanical Boundary Conditions................90

Figure 4.38 Illustration of Mechanical Boundary Conditions..................................91

Figure 5.1 Temperature Contour Plot, Integrated SiC Mini-contact.........................92

Figure 5.2 Location of Maximum Displacement Plot, Integrated SiC Mini-

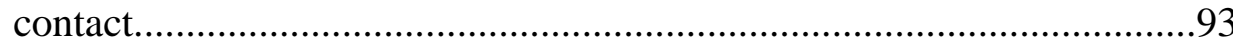

Figure 5.3 Location of Maximum Displacement Plot, Integrated SiC Mini-

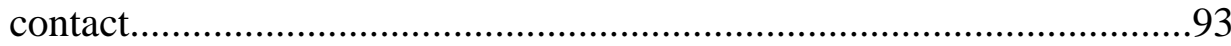

Figure 5.4 Illustration of Thermal Expansion Effect..............................................94

Figure 5.5 Von-Mises Plastic Strain Plot - a, Solder Joint at Mini-contact/TE Cooler Header Interface and b, Solder Joint at TE elements/Copper Straps

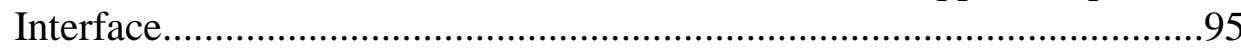

Figure 5.6 $1^{\text {st }}$ Pr. Stress Contour Plot - TE Elements, Integrated SiC Mini-

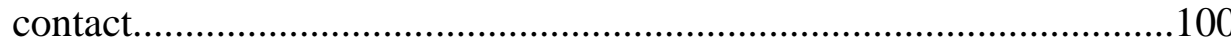

Figure 5.7 $2^{\text {nd }}$ Pr. Stress Contour Plot - TE Elements, Integrated SiC Mini-

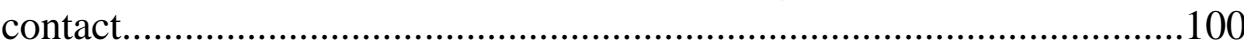

Figure $5.83^{\text {rd }}$ Pr. Stress Contour Plot - TE Elements, Integrated SiC Minicontact. .100

Figure 5.9 $1^{\text {st }}$ Pr. Stress Plot - TE Cooler Headers, Integrated SiC Mini-contact......101

Figure 5.10 $2^{\text {nd }}$ Pr. Stress Plot - TE Cooler Headers, Integrated SiC Mini-

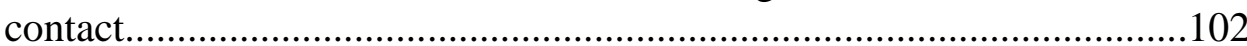

Figure 5.11 $3^{\text {rd }}$ Pr. Stress Plot - TE Cooler Headers, Integrated SiC Mini-contact...102 
Figure 5.12 $1^{\text {st }}$ Pr. Stress Plot - GaN on SiC substrate, Integrated SiC Minicontact.

Figure $5.132^{\text {nd }}$ Pr. Stress Plot - GaN on SiC substrate, Integrated SiC Minicontact.

Figure 5.14 $3^{\text {rd }}$ Pr. Stress Plot - GaN on SiC substrate, Integrated SiC Minicontact... 104

Figure 5.15 Temperature Contour Plot, T-shaped Copper Mini-contact. .105

Figure 5.16 Displacement Contour Plot, T-shaped Copper Mini-contact. 105

Figure 5.17 Pl. Strain - Solder Joint: SiC/Mini-contact, T-shaped Cu contact...........106

Figure 5.18 Pl. Strain - Solder Joint: TE Cooler/Mini-contact, T-shaped Cu contact.

Figure 5.19 Pl. Strain - Solder Joint in TE Cooler.

Figure 5.20 $1^{\text {st }}$ Principal Stress Plot - TE Elements, T-shaped Cu contact.

Figure 5.21 $2^{\text {nd }}$ Principal Stress Plot - TE Elements, T-shaped Cu contact. 110

Figure 5.22 $3^{\text {rd }}$ Principal Stress Plot - TE Elements, T-shaped Cu contact. 110

Figure 5.23 $1^{\text {st }}$ Principal Stress Plot - TE Cooler Headers, T-shaped Cu contact.......111

Figure 5.24 $2^{\text {nd }}$ Principal Stress Plot - TE Cooler Headers, T-shaped Cu contact.....111 Figure 5.25 $3^{\text {rd }}$ Principal Stress Plot - TE Cooler Headers, T-shaped Cu contact.....111

Figure 5.26 $1^{\text {st }}$ Principal Stress Plot - GaN on SiC Device, T-shaped Cu contact.....112 Figure 5.27 $2^{\text {nd }}$ Principal Stress Plot - GaN on SiC Device, T-shaped Cu contact....113 Figure 5.28 $3^{\text {rd }}$ Principal Stress Pot - GaN on SiC Device, T-shaped Cu contact.....113 Figure 5.29 Temperature Contour Plot, Lofted Cu Mini-contact.............................114

Figure 5.30 Displacement Contour Plot, Lofted Cu Mini-contact............................115

Figure 5.31 Pl. Strain - Solder Joint: Mini-contact/TE Cooler, Lofted Cu Minicontact.

Figure 5.32 Pl. Strain - Solder Joint inside TE Cooler, Lofted Cu Mini-contact......117

Figure 5.33 $1^{\text {st }}$ Principal Stress Plot - TE Elements, Lofted Cu contact....................118

Figure 5.34 $2^{\text {nd }}$ Principal Stress Plot - TE Elements, Lofted Cu contact....................118

Figure 5.35 $3^{\text {rd }}$ Principal Stress Plot - TE Elements, Lofted Cu contact....................119

Figure 5.36 $1^{\text {st }}$ Principal Stress Plot - TE Cooler Headers, Lofted Cu contact...........120

Figure 5.37 $2^{\text {nd }}$ Principal Stress Plot - TE Cooler Headers, Lofted $\mathrm{Cu}$ contact..........120

Figure $5.383^{\text {rd }}$ Principal Stress Plot - TE Cooler Headers, Lofted Cu contact..........120

Figure 5.39 $1^{\text {st }}$ Principal Stress Plot - GaN on SiC substrate, Lofted Cu contact......121

Figure $5.402^{\text {nd }}$ Principal Stress Plot - GaN on SiC substrate, Lofted Cu contact.....121

Figure 5.41 $3^{\text {rd }}$ Principal Stress Pot - GaN on SiC substrate, Lofted Cu contact........122

Figure 5.42 Temperature Contour Plot, Taper Cu Mini-contact.

Figure 5.43 Displacement Contour Plot, Taper Cu Mini-contact.

Figure 5.44 Pl. Strain - Solder Joint: Mini-contact/TE Cooler, Taper Cu Minicontact......

Figure 5.45 Pl. Strain - Solder Joint inside TE Cooler, Taper Cu Mini-contact........126

Figure 5.46 $1^{\text {st }}$ Principal Stress Plot - TE Elements/Headers, Taper Cu Minicontact......

Figure 5.47 $2^{\text {nd }}$ Principal Stress Plot - TE Elements/Headers, Taper Cu Minicontact.

Figure $5.483^{\text {rd }}$ Principal Stress Plot - TE Elements/Headers, Taper $\mathrm{Cu}$ Minicontact. 
Figure 5.49 $1^{\text {st }}$ Principal Stress Plot - SiC/GaN Device, Taper Cu Minicontact..

Figure 5.50 $2^{\text {nd }}$ Principal Stress Plot - SiC/GaN Device, Taper Cu Minicontact.

Figure 5.51 $3^{\text {rd }}$ Principal Stress Pot - SiC/GaN Device, Taper Cu Mini-contact.......130

Figure 5.52 Temperature Contour Plot, T-shaped Diamond Mini-contact................131

Figure 5.53 Displacement Contour Plot 1, T-shaped Diamond Mini-contact...........132

Figure 5.54 Pl. Strain - Solder Joint: Mini-contact/SiC Substrate Interface, T-shaped

Diamond Mini-contact

Figure 5.55 Pl. Strain - Solder Joint: Cooler/Mini-contact Integration, T-shaped

Diamond Mini-contact

Figure 5.56 Plastic Strain Plot - Solder Joint: Thermo-electric Cooler, T-shaped Diamond Mini-contact

Figure $5.571^{\text {st }}$ Principal Stress Plot 1 - Mini-contact, T-shaped Diamond contact.

Figure $5.581^{\text {st }}$ Principal Stress Plot 2 - Mini-contact, T-shaped Diamond contact.

Figure $5.591^{\text {st }}$ Principal Stress Plot 3 - Mini-contact, T-shaped Diamond contact..

Figure $5.602^{\text {nd }}$ Principal Stress Plot - Mini-contact, T-shaped Diamond contact......136

Figure 5.61 $3^{\text {rd }}$ Principal Stress Plot 1 - Mini-contact, T-shaped Diamond contact.

Figure $5.623^{\text {rd }}$ Principal Stress Plot 2 - Mini-contact, T-shaped Diamond contact.

Figure $5.631^{\text {st }}$ Principal Stress Plot - TE Elements, T-shaped Diamond contact.....137

Figure 5.64 $2^{\text {nd }}$ Principal Stress Plot - TE Elements, T-shaped Diamond contact.....137

Figure 5.65 $3^{\text {rd }}$ Principal Stress Plot - TE Elements, T-shaped Diamond contact.....137

Figure 5.66 $1^{\text {st }}$ Principal Stress Plot - TE Cooler Headers, T-shaped Diamond

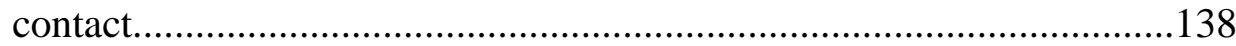

Figure 5.67 $2^{\text {nd }}$ Principal Stress Plot - TE Cooler Headers, T-shaped Diamond

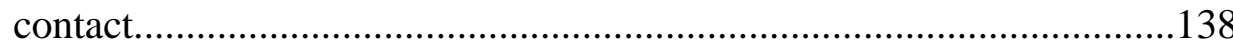

Figure $5.683^{\text {rd }}$ Principal Stress Plot - TE Cooler Headers, T-shaped Diamond

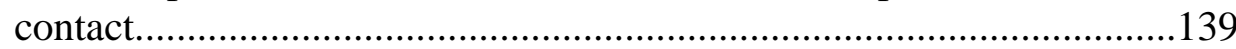

Figure 5.69 $1^{\text {st }}$ Principal Stress Plot - SiC/GaN Device, T-shaped Diamond contact.

Figure 5.70 $2^{\text {nd }}$ Principal Stress Plot - GaN on SiC substrate, T-shaped Diamond contact.

Figure 5.71 $3^{\text {rd }}$ Principal Stress Pot - GaN on SiC substrate, T-shaped Diamond contact.

Figure 5.72 Temperature Contour Plot, Lofted Diamond Mini-contact

Figure 5.73 Displacement Contour Plot, Lofted Diamond Mini-contact. 142

Figure 5.74 Pl. Strain in Solder: Chip Level Integration, Lofted Diamond Minicontact.

Figure 5.75 Pl. Strain in Solder: Mini-contact/Cooler Integration Level, Lofted Diamond Mini-contact.

Figure 5.76 Pl. Strain in Solder - TE Cooler, Lofted Diamond Mini-contact. 
Figure 5.77 $1^{\text {st }}$ Principal Stress Plot 1 - Mini-contact, Lofted Diamond Minicontact.....

Figure $5.781^{\text {st }}$ Principal Stress Plot 2 - Mini-contact, Lofted Diamond Minicontact.

Figure 5.79 $1^{\text {st }}$ Principal Stress Plot - TE Elements, Lofted Diamond Minicontact......

Figure $5.802^{\text {nd }}$ Principal Stress Plot - TE Elements, Lofted Diamond Minicontact......

Figure 5.81 $3^{\text {rd }}$ Principal Stress Plot - TE Elements, Lofted Diamond Minicontact...

Figure 5.82 $1^{\text {st }}$ Principal Stress Plot - TE Cooler Headers, Lofted Diamond Minicontact......

Figure $5.832^{\text {nd }}$ Principal Stress Plot - TE Cooler Headers, Lofted Diamond Minicontact.....

Figure 5.84 $3^{\text {rd }}$ Principal Stress Plot - TE Cooler Headers, Lofted Diamond Minicontact.

Figure 5.85 $1^{\text {st }}$ Principal Stress Plot - GaN on SiC substrate, Lofted Diamond Minicontact......

Figure 5.86 $2^{\text {nd }}$ Principal Stress Plot - GaN on SiC substrate, Lofted Diamond Minicontact.

Figure $5.873^{\text {rd }}$ Principal Stress Pot - GaN on SiC substrate, Lofted Diamond Mini-

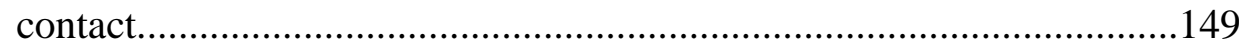

Figure 5.88 Temperature Contour Plot, Taper Diamond Mini-contact.....................150

Figure 5.89 Displacement Contour Plot, Taper Diamond Mini-contact....................150

Figure 5.90 Pl. Strain - Solder Joint: Chip Level Integration, Taper Diamond Minicontact.

Figure 5.91 Pl. Strain - Solder Joint - Cooler/Mini-contact Integration, Taper Diamond Mini-contact.

Figure 5.92 $1^{\text {st }}$ Principal Stress Plot 1 - Mini-contact, Taper Diamond Minicontact.

Figure $5.931^{\text {st }}$ Principal Stress Plot 2 - Mini-contact, Taper Diamond Minicontact..

Figure $5.942^{\text {nd }}$ Principal Stress Plot - TE Elements, Taper Diamond Minicontact..

Figure $5.953^{\text {rd }}$ Principal Stress Plot - TE Elements, Taper Diamond Minicontact..

Figure 5.96 $1^{\text {st }}$ Principal Stress Plot - TE Elements, Taper Diamond Minicontact..

Figure 5.97 $2^{\text {nd }}$ Principal Stress Plot - TE Elements, Taper Diamond Minicontact.

Figure $5.983^{\text {rd }}$ Principal Stress Plot - TE Elements, Taper Diamond Minicontact.

Figure 5.99 $1^{\text {st }}$ Principal Stress Plot - TE Headers, Taper Diamond Mini-contact....156 Figure 5.100 $2^{\text {nd }}$ Principal Stress Plot - TE Headers, Taper Diamond Minicontact. 
Figure 5.101 $3^{\text {rd }}$ Principal Stress Plot - TE Headers, Taper Diamond Mini-

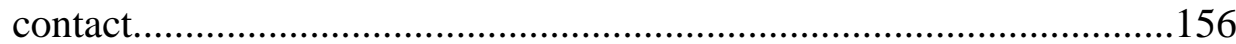

Figure 5.102 $1^{\text {st }}$ Principal Stress Plot - SiC/GaN Device, Taper Diamond Mini-

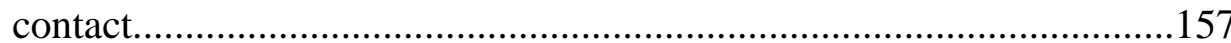

Figure 5.103 $2^{\text {nd }}$ Principal Stress Plot - SiC/GaN Device, Taper Diamond Mini-

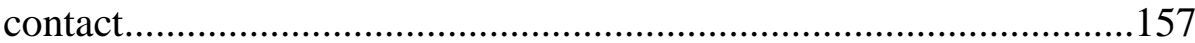

Figure 5.104 $3^{\text {rd }}$ Principal Stress Pot - SiC/GaN Device, Taper Diamond Mini-

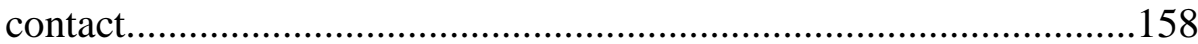

Figure 5.105 C.T.E. Mismatch: SiC - SAC305 - AlN...........................................159

Figure 5.106 C.T.E. Mismatch across TE Cooler..................................................159

Figure 5.107 C.T.E. Mismatch: SiC - SAC305 - Copper......................................... 160

Figure 5.108 C.T.E. Mismatch: Copper - SAC305 - AlN....................................... 160

Figure 5.109 C.T.E. Mismatch: SiC - SAC305 - Diamond.......................................161

Figure 5.110 C.T.E. Mismatch: Diamond - SAC305 - AlN.....................................161

Figure 5.111 T-shaped, Lofted and Taper Mini-contact Model...............................162

Figure 6.1 Temperature Contour Plot, T-shaped, Silver-Diamond Composite..........165

Figure 6.2 Displacement Contour Plot, T-shaped, Silver-Diamond Composite........166

Figure 6.3 Pl. Strain - Solder Joint: Thermo-electric Cooler.....................................167

Figure 6.4 Von-Mises Stress Distribution: Mini-contact Region...............................168

Figure 6.5 Nodal Temperature Contour Plot, Lofted, Silver-Diamond

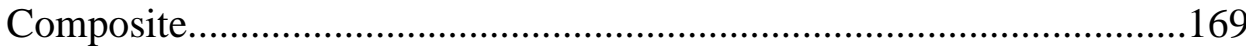

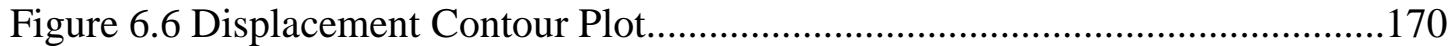

Figure 6.7 Pl. Strain - Solder Joint Thermo-electric Cooler....................................170

Figure 6.8 Von-Mises Stress Distribution: Mini-contact Region.............................171

Figure 6.9 Temperature Contour Plot across Model............................................172

Figure 6.10 Displacement Contour Plot.................................................................... 173

Figure 6.11 Von-Mises Stress Contour Plot............................................................... 174

Figure 7.1 Manifold Micro-channel Cooling Schematic.........................................175

Figure 7.2 Unit Cell Model................................................................................176

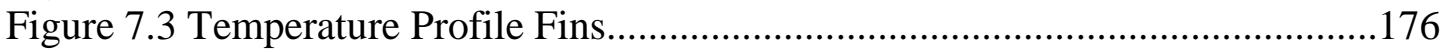

Figure 7.4 Maximum Principal Stress Plot - Micro-fin Corner................................177

Figure 7.5 Stress Concentration Factor............................................................ 178 


\section{Chapter 1: Introduction and Motivation}

There is an ever-present drive in the electronics industry to develop smaller products with enhanced functionalities. This is true not only in consumer electronics but in power electronics as well. In the case of power electronics, this translates into greater power throughput in ever smaller devices and packages. An example of this is the GaN on SiC (High Electron Mobility Transistor) HEMT devices used in aerospace electronics - (Silicon Carbide/Gallium Nitride electronics). Even with increases in device efficiency, this leads to higher densities of power loss and heat generation. This necessitates development of new cooling technologies to dissipate the high levels of heat generated through the electronics. One of these new technologies involves integrating a two-phase manifold-microchannel cooler combined with a thermoelectric spot cooler directly on the backside of a high power device. This technology aims to use the two-phase manifold microchannel cooler to dissipate $1 \mathrm{~kW} / \mathrm{cm}^{2}$ of heat flux across the device while also removing up to $5 \mathrm{~kW} / \mathrm{cm}^{2}$ of heat flux generated at the device hot-spot using the thermoelectric cooler. To enhance the cooling power of the thermoelectric device, a Mini-contact will be used to spread the heat from the hot spot to the larger thermoelectric. The objective of this study is to evaluate the structural reliability of this integrated cooler, including the two-phase manifold microchannel cooler, the thermoelectric device, the Mini-contact, and the integration on the backside of the wide bandgap power semiconductor device. Specifically, the study focuses on thermo-mechanical failure of the attachment (adhesive solder layer) between the thermoelectric cooler and Mini-contact, Minicontact and die, and manifold and microchannel for three types of Mini-contact 
geometries (viz. T-shaped, lofted, and tapered). Failure of attachment within the thermoelectric device has also been performed in this study. Suggestions for material selection for the Mini-contact have also been made based on relative structural reliability, where the driving force for thermo-mechanical failure is the difference in coefficients of thermal expansion of various materials used and the temperature gradient due to the heat generation of $5 \mathrm{k} \mathrm{W} / \mathrm{cm}^{2}$ at the device hot-spot.

\subsection{Power Electronics Materials: GaN on Si, GaN on SiC}

"Power electronics is the application of solid-state electronics for the control and conversion of electrical power." [1] These devices convert electricity in the form (i.e., voltage, current, and frequency) at which it is generated and convert it to the form (i.e., voltage, current, and frequency) that is optimized for transmission or for use in an application. Examples of modern power electronics include semiconductor switching devices such as diodes, thyristors and transistors. In consumer electronics (e.g. television sets, personal computers, battery chargers), the AC/DC converter (rectifier), which is composed of switching devices, passive components (e.g., capacitors) and control circuitry, is the most common power electronics system one can find.

Today, power electronics use is widespread across many application sectors, of which defense and automotive electronics are two of the leading users. These applications have pushed power electronics to the temperature limit of silicon devices, which is found to be around $150^{\circ} \mathrm{C}-175^{\circ} \mathrm{C}$. Hence, there is need for alternative materials, which can operate reliably at high temperatures, without significant degradation. [2] Gallium Nitride (GaN) and Silicon Carbide ( $\mathrm{SiC})$ wide 
band-gap semiconductor devices are being researched for their ability to operate at high temperature. Some of the other advantages resulting from the use of these materials instead of silicon include: increased power density, faster switching speed, and higher reverse breakdown voltage. Devices which have been fabricated out of SiC include MOSFETS, JFETs, thyristors, and diodes. In a recent study [1], a SiC schottky diode was shown to operate at temperatures up to $700^{\circ} \mathrm{C}$ and a MOSFET up to $650^{\circ} \mathrm{C}$. On the other hand, temperature degradation in silicon devices occurs at temperatures greater than $175^{\circ} \mathrm{C}$.

\subsection{What is Thermally Integrated Power Electronics Packaging?}

The invention of transistor is considered as the beginning of the information revolution, as it ushered in the widespread usage of electronic products by shrinking product size considerably over that for earlier vacuum tube electronics. [3] Later, in the 1960s, integrated circuit technology extended system functionality while reducing the product size even more by integrating hundreds of transistors on a single semiconductor chip. Integrated circuits (ICs), based on microelectronic devices, form the basis of all modern electronic products. Increasing product functionality along with performance has been the driving force for the further development of electronics, which aims to integrate billions and trillions of transistors on a single semiconductor chip. [4] However, one needs to understand that in order to fulfill these demands; packaging materials and thermal management solutions are needed to handle the large quantities of power dissipated by these devices during operation.

Nowhere is this combination of increasing power loss and decreasing package size more true than for power electronics. Here coolers are being designed into the 
package in order to provide direct chip cooling. These thermally integrated packages provide mechanical protection, supply of power, and cooling of power electronics switch or components along with provision of electrical and mechanical connection between the power electronic part and outside world. The main challenge for the package is to provide all crucial functions required by the microelectronic part without limiting the performance of the part and while withstanding the large thermomechanical stresses that such a package necessarily incurs.

\subsubsection{Design for Reliability}

A product is said to be designed with high reliability, when it performs its intended function under the given loading and environmental conditions for the desired lifespan. $[3,5]$ In order to ensure that the electronic systems package will be reliable over an extended period of time, two steps need to be followed. First, design the systems package up-front for reliability. Second, after the system is designed, fabricated and assembled, conduct accelerated qualification testing on the systems to confirm their reliability.

Designing for reliability includes determining the potential failure mechanisms, and then designing the product and selecting materials and processes that minimize or eliminate the susceptibility to failure. In general, the methodology for design for reliability can be achieved by either reducing stresses that will lead to failure in the device via change in the package geometry and dimensions, selection of an alternative material, appropriate protection or encapsulation, or by combination of all of these or by increasing the strength of the component typically by selection of an appropriate material which can withstand the stresses due to loading. Hence, the 
underlying principle of design for reliability is that reliability is designed into an electronics system/product upfront before the system/product is built, thus eliminating costly design-test-fix iterative cycles.

After the system is built and assembled, it is subjected subsequently to accelerated testing under conditions of thermal cycling, temperature and humidity cycling or power cycling for short periods of time by applying higher load levels (e.g. temperature, humidity, voltage, pressure) than it would experience in the field to accelerate the failure process.

\subsubsection{Fundamentals of Thermal Management}

It is essential to cool the electronics and remove the heat generated by electronic devices, because if the heat is not removed, the temperature of the electronic device will rise until it reaches a value at which the electronic component stops operating and ultimately, is destroyed. In order to remove the heat, the component can be placed in contact with a lower temperature solid or fluid. As the heat is removed, the temperature rise in the solid body is moderated and it asymptotically approaches a steady-state value.

When a solid body is at steady-state temperature, all the heat generated inside the component is dissipated to the surrounding structure and/or fluid. In order to achieve a smaller temperature rise above the ambient temperature in steady state, a higher heat transfer coefficient method must be used, such as high velocity air jets rather natural convection or boiling rather than low velocity liquid flow. Last, but not least, for a successful thermal packaging solution, we should focus on careful 
combination of materials and heat transfer mechanisms to stabilize the component temperature at a tolerable level. [3-7]

Reliability of an electronic product is defined as the probability that the product will perform its intended function under the intended operational and environmental load conditions for a desired application-specific period of time. An electronic product can work reliably for many years, especially, when they operate at or near room temperature. However, integrated circuits often become more susceptible to failure after prolonged exposure to elevated temperatures. This accelerated failure rate results from mechanical creep in materials used for bonding (solder), chemical reactions, inter-diffusion, etc. Hence, it has traditionally been postulated that there is an exponential relationship between operating temperature and decreased product reliability. Therefore, thermal management is essential in order to ensure better reliability.

As per the commonly accepted nomenclature for representing different packaging levels used in the industry today, the packaging levels can be defined as follows. A package which houses and protects chip (chip package) is referred to as first level package. The second level package constitutes the printed wiring board (PWB) for chip-to-chip communication. The third level package constitutes the motherboard (backplane) and full assembly.

The mechanism and methods of heat transfer vary substantially from one packaging level to another. At package level 1, with the main concern is how heat transfers or conducts from chip to the package surface and then into the printed wiring board. In order to lower the chip temperature, reduction of thermal resistance 
between the silicon die and the outer surface of package should be considered. A variety of passive cooling techniques are available, which can be used for reduction of thermal resistance. These include: high-conductivity adhesive, greases, phase change materials, high conductivity molding compound, heat spreader, and heat slug, Thermal performance can be improved using die-attach adhesives with diamond, silver, high conductivity fill material, thermal greases and phase-change materials, which soften at operating temperature to conform to the surface of chip. Heat sinks can be attached to the surface of package to create additional surface area for heat removal by convection. Convection can be achieved by circulation of air on the surface of heat sink with the help of a fan. Very high power electronics can be cooled by using a heat pipe attached directly to the surface of chip, impingement of high velocity air jets or by immersion in a dielectric liquid. [3-7]

At package level 2 , heat removal occurs mainly by conduction in printed wiring board followed by convection to the ambient air. Printed wiring boards with thick, high conductivity power and ground planes and/or embedded heat pipes can provide improved thermal spreading at this packaging level. Use of metal substrates with insulation could also be considered for better thermal management.

\subsection{Electronics Cooling Methods}

This section highlights fundamental cooling mechanisms that can be applied to dissipate heat from an electronics device and consequently keep the temperature of the device below its maximum allowable temperature. 


\subsubsection{Heat Sinks}

Thermal resistance due to convection is inversely proportional to the product of heat transfer coefficient and the heat transfer surface area. [9] Hence, it can be inferred that in order to reduce the thermal resistance, one can either increase the effective cooling area or increase the heat transfer coefficient of the coolant. For most common thermal management problems, heat transfer coefficient is function of the flow conditions, which are fixed. Alternatively, increase in the heat transfer area seems to be the most feasible or practical way to reduce thermal resistance in the cooling network. The same can be achieved with use of extended surfaces or fins, as shown in the figure below.

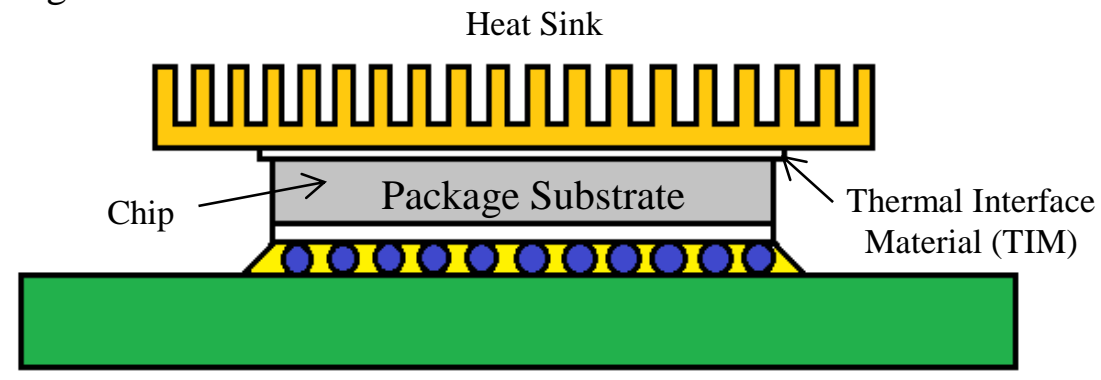

Figure 1.1: Heat Removal through use of Heat Sink, [9]

As per Fourier's law of cooling and considering when the base of fin is above ambient temperature, as away from the base of the fin, there will be a decrease in temperature with the associated heat flow in the fin. [7]

As displayed in the figure 1.1 above, arrays of fins or extended surfaces in the form of heat sinks or coolers are frequently used for thermal management of electronic equipment. Heat sink thermal resistance value is generally provided by the manufacturer for a range of flow rates. A large variety of heat sinks are available for cooling of electronics, with most common being extruded heat sinks. 


\subsubsection{Thermoelectric Phenomena}

Thermoelectric coolers (TEC) are based on the Peltier Effect, and essentially act as solid-state heat pumps. When a potential is applied across the two junctions, heat is absorbed at one junction, and expelled at the other, in proportion to the electric current.

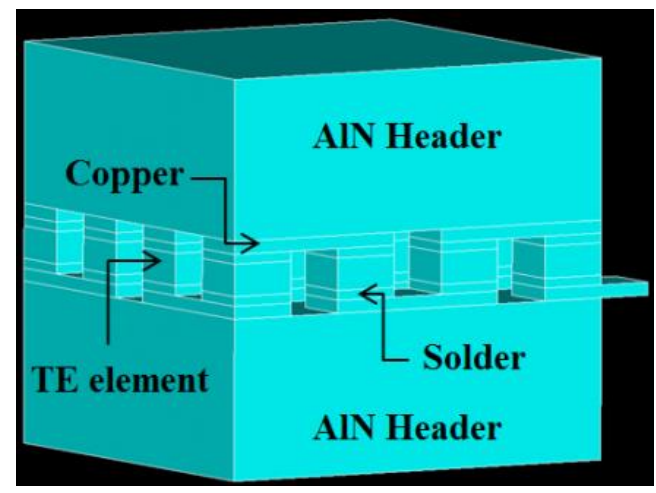

Figure 1.2: Schematic Diagram of a Thermoelectric Cooler

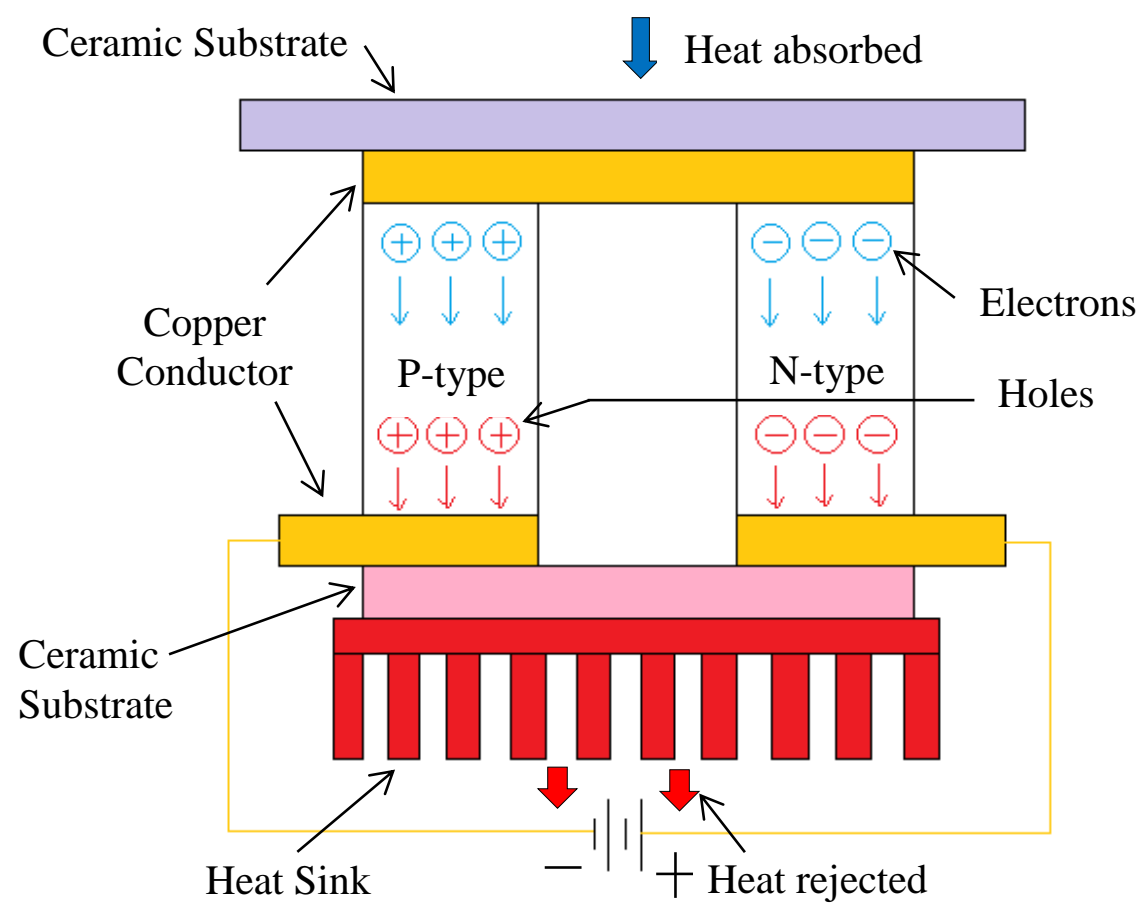

Figure 1.3: Explanation of Thermoelectric Phenomena, $[10,11]$ 
The Peltier effect is observed with most material combinations. It describes the process by which one junction of a bi-material couple is cooled and the other is heated, when electric current is maintained in a circuit of material consisting two dissimilar conductors. The thermoelectric effect becomes even more significant when a circuit consists of dissimilar semiconductors. $[10,11]$

When a current is supplied, electrons are transported from the p-side of the junction to the n-side, where they are elevated to a higher energy state. This causes heat to be absorbed and consequently results in cooling the surrounding area. When the electrons are transported from the p-side back to the n-side, they release heat. Semiconductor materials used to make TEC include bismuth telluride $\left(\mathrm{Bi}_{2} \mathrm{Te}_{3}\right)$, lead telluride $(\mathrm{PbTe})$ and silicon germanium ( $\mathrm{SiGe})$. A TEC device is constructed by placing several hundred thermocouples electrically in series, and thermally in parallel, between two pieces of metallized, thermally conductive ceramic acting as an electrical insulator. The thermocouples are inter-connected to the metallized ceramic via solder. The ceramic substrates and thermocouples are brittle in nature. However, failure is typically observed at the interface between the metallization and the ceramic or at the solder interconnection. In order to achieve continuous cooling at the low temperature side of the TEC, heat absorbed at the cold side and subsequently pumped to the hot side should be removed from the hot side using a secondary heat transport mechanism, such as a heat sink. $[10,11]$

\subsubsection{Hot Spot Cooling and related Reliability Issues}

A hot spot may be defined as a localized region of high heat flux on a semiconductor device (e.g., IGBT, microprocessor). In general, a temperature 
differential across a microprocessor die can be observed to vary from 5 to $30 \mathrm{~K}$ due to large variations in heat flux density. These regions of high heat flux often limit the reliability and performance of the device. Traditional chip-scale thermal management solutions designed to keep hot spots below a critical temperature can needlessly overcool the rest of device and increase the heat-sink load. One of the promising techniques to reduce the temperature of highly localized, high heat flux hot spots is through the use of embedded thermoelectric cooling (eTEC). However, with mitigation of high heat flux in localized concentrated regions, associated thermomechanical stress and strain effects will need to be addressed.

Hot spots can be cooled by either spot cooling or heat spreading by any number of available passive or active cooling methods. [12] Examples of cooling methods include: high thermal conductivity heat spreading materials (diamond), microchannel fluidic coolers, or two-phase cooling techniques such as jet/spray cooling or heat pipes. One of the drawbacks of using high thermal conductivity heat spreaders is their high cost and difficulty with integration to silicon. Two phase cooling systems have a limitation of difficulty in manufacturing and modeling.

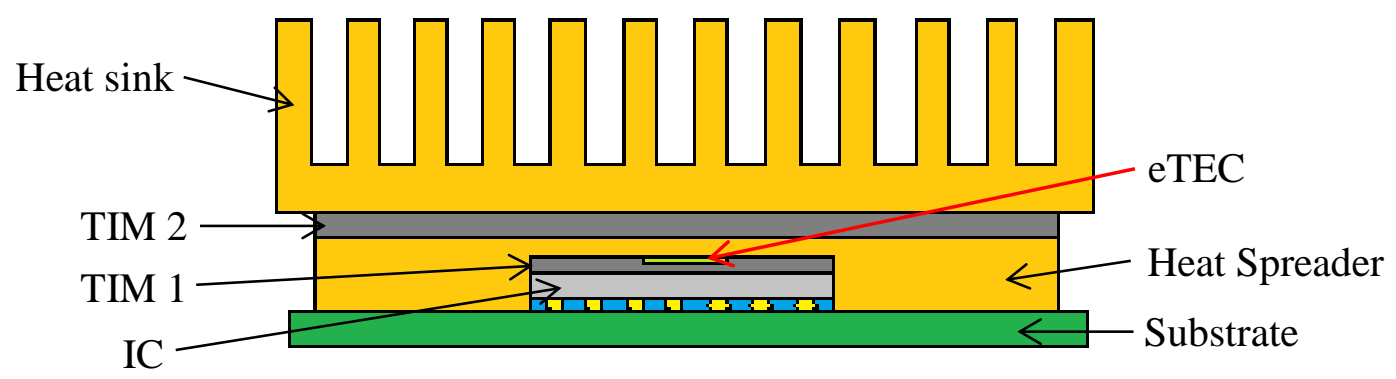

Figure 1.4: Flip Chip Package with eTEC mounted on Heat Spreader, [12]

It is important to realize that the reliability of a thermal management solution is an important consideration. Thermoelectric coolers have an advantage of no 
moving parts. However, they should be able to withstand large heat flux densities and thermal gradients.

In the research presented by Alley et. al. [13] on the reliability testing of thinfilm super lattice thermoelectric devices, thin-film super lattice films were subjected to high power and temperature on/off cycle testing, which resulted in thermomechanical stress generation in the film. Under the above stressed conditions, they were found to have little change in thermoelectric properties after over 50,000 cycles.

From a reliability standpoint, it is important to consider the thermomechanical properties of the various materials present in a TEC device stack. Essentially, a TE cooler consists of thermoelectric elements placed between two metallized ceramic substrates. [14] These elements are interconnected to the metallized ceramic substrates via solder attach. The attachment of different material layers, which differ in their Coefficient of Thermal Expansion (C.T.E.) values, will result in generation of thermo-mechanical stresses. Another driving force for thermomechanical stresses will be the presence of a thermal gradient across the device. As stated in the research $[13,14]$, one can overcome the thermo-mechanical stress effects by using a compliant interface material between the TE and target device. Usually, there are thermal interface materials available for interconnection between the substrate and heat sink or thermal spreader, which can accommodate the stress effect. This will ultimately lead to reduction of thermal stress generation at both TE device and substrate level. 


\section{Chapter 2: Reliability Theory and Physics of Failure Models}

\subsection{Physics of Failure Perspective of Reliability}

"Reliability is the ability of a product to properly function, within specified performance limits, for a specified period of time, under the life cycle application conditions." [15]

Stated alternatively, in order for the product to be reliable (product doesn't fail); it must function under the desired operational and environmental life cycle conditions (including mechanical, thermal and electrical conditions) in a manner to meet the user requirements or specified performance limits for a useful life (time can be expressed in terms of time, miles, cycles, or any sequence or sequencing index). [15]

Component reliability involves the study of both reliability physics and reliability statistics. Both provide an important contribution to better understanding the ways in which components fail, and how the failures are developing in time. They also provide a background for understanding and assessing the real-world failure patterns of component reliability that come to us from field failure studies.

Traditionally, the effort of the reliability engineers has been concentrated on establishing lifetime patterns for individual component types (or for individual failure mechanisms). Reliability is a collective name for those measures of quality that reflect the effect of time in the storage or the use of product, as distinct from those measures that describe the state of the product at the time of delivery (i.e. manufacturing quality). $[4,6,15]$ 
For random failures, the reliability of individual parts is typically characterized by a failure rate $(\lambda)$, which defines the fraction of parts that fail over a fixed time unit. For a constant failure rate, the reliability of an isolated constructive element is $\exp (-\lambda t)$, and consequently, the reliability of $n$ elements is $\exp (-n \lambda t)$.

\subsection{Physics of Failure - POF process for Reliability Assessment}

For failures which are not random but for which the root cause can be determined, it is more accurate to use a physics-of-failure (PoF) approach. The basic premise of PoF is that failures can be traced to a fundamental degradation mechanism (e.g. chemical, electrical, mechanical, thermo-mechanical) that is operative for the design used and the environment in which it is expected to operate. Physics-offailure models contain the following two key elements: 1) a load transformation model that converts the operational or environmental loads to stress or strain levels based on the component or system design or hardware configuration, and 2) an empirical damage model that determines the time to failure for the component under that level of stress or strain.

Physics of Failure (PoF) reliability engineers are interested in understanding and controlling the root causes underlying individual failures at all phases of the lifecycle. $[18,19]$ This is done through systematic and detailed assessment of influence of hardware configuration and life-cycle stresses on root-cause failure mechanisms in the materials at potential failure sites.

The PoF process for reliability assessment can be divided into three main steps, which basically include (1) Inputs, (2) Analysis and (3) Outputs. The inputs to PoF reliability analysis include: hardware configuration (e.g., materials, geometry), 
life cycle load condition (e.g. transportation, storage, handling and application), operational loads (e.g., power dissipation, voltage, current, frequency, duty cycle), and environmental loads (e.g. temperature, relative humidity, shock). [18]

The reliability analysis process involves both virtual analysis or simulation and actual physical testing i.e., accelerated life testing). These are used to determine stresses and damage at failure sites under life-cycle loading. The reliability analysis provides design margins for each relevant failure mechanism at each failure site, stress margins for overstress mechanisms, and life margins for wear out mechanisms. For aggregation to the system level; we can use reliability block diagrams, Monte Carlo simulations, or Bayesian updates with field/test data, if any. Reliability analysis may also include sensitivity analysis, where we evaluate the sensitivity of the product durability to changes in application, design, and manufacturing process windows.. The final output of the reliability analysis process is a ranking of potential failure mechanisms and sites that can be used to make design tradeoffs, provide risk mitigation solutions, or employ for prognostic health monitoring. [18, 19]

\subsection{Thermo-Mechanical Reliability}

Two of the most common failure mechanisms for power electronic devices are thermo-mechanical fatigue of the attachment materials under thermal cycling stresses and thermo-mechanical fracture of the die or substrate. To understand these failures it is important to look at how these thermal stresses arise. 


\subsubsection{Thermal Expansion}

Most solid materials expand on heating and contract when cooled. [3] At an atomic level, thermal expansion can be understood as an increase in the average distance between the atoms with increasing temperature as can be derived from the Lenard-Jones potential energy versus interatomic spacing model for a solid material. Considering a single dimension, the engineering strain resulting from this change in length with temperature for a solid material may be expressed as follows:

$$
\begin{gathered}
\frac{l_{f}-l_{0}}{l_{0}}=\alpha_{l}\left(T_{f}-T_{0}\right) \\
\frac{\Delta l}{l_{0}}=\alpha_{l} \Delta T
\end{gathered}
$$

where, $l_{0}$ and $l_{f}$ represent the initial and final lengths with the temperature change from $T_{0}$ to $T_{f}$. The parameter $\alpha_{l}$ is called the linear coefficient of thermal expansion. The coefficient of thermal expansion is a material property that is indicative of the extent to which a material expands upon heating and has units of reciprocal temperature (i.e. ${ }^{\circ} \mathrm{C}^{-1}$ or ${ }^{\circ} \mathrm{F}^{-1}$ ). As heating or cooling affects all the dimensions of a body, with a resultant change in volume, the volume changes with temperature may be computed with the help of following equation.

$$
\frac{\Delta V}{V_{0}}=\alpha_{v} \Delta T
$$

In the above equation, $\Delta V$ and $V_{0}$ represent the volume change and the original volume respectively and $\alpha_{v}$ is the volume coefficient of thermal expansion. . $\alpha_{v}=3 \alpha_{l}$ for materials which have isotropic thermal expansion coefficient, however, 
in many materials $\alpha_{v}$ is anisotropic in nature, which means that this value depends on the crystallographic direction along which it is measured.

\subsubsection{Thermal Mismatch}

In an electronics package, it's a very common scenario that materials with different thermal expansion coefficients are assembled together. During manufacturing and operation, the package goes through various temperature cycles, which result in differential thermal expansion of the materials used in the package. Since the assembled materials are constrained by each other in the package, the materials cannot expand freely, resulting in significant thermal mismatch stress generation inside the package. [3 - 6]

\subsubsection{Thermal Stresses}

The thermal stresses generated in an electronic package can lead to excessive elastic deformation, plastic yielding or fracture. [3 - 6] The simplest example of the generation of thermal stress is shown using a homogeneous and isotropic solid rod that is heated or cooled uniformly (i.e. no spatial temperature gradients are imposed). If the rod is free to expand or contract, there will be no stresses generated. However, if the axial motion of the rod is restrained by rigid end supports, thermal stresses will be generated. As a result of temperature change from $T_{0}-T_{f}$, the amount of stress generated is given by the equation,

$$
\sigma=E \alpha_{l}\left(T_{0}-T_{f}\right)=E \alpha_{l} \Delta T
$$

where, E is Young's modulus of elasticity and $\alpha_{l}$ is the linear coefficient of thermal expansion. When the rod is heated $\left(T_{f}>T_{0}\right)$, compressive stresses are generated, 
while if the rod is cooled, $\left(T_{f}<T_{0}\right)$, tensile stresses will be imposed. The stress in the above equation is same as the stress that would be required to elastically compress or elongate the rod specimen back to its original length after it has been allowed to freely expand or contract from temperature: $T_{0}-T_{f}$.

\subsubsection{Stresses Generated due to Temperature Gradients}

When a solid body is heated or cooled, the internal temperature distribution will depend on its size and shape, thermal conductivity of the material, and the rate of temperature change. These internal temperature gradients can cause thermal stresses to be generated during rapid heating or cooling of the solid. Rapid heating or cooling causes the temperature of the outside to change more quickly than the temperature of the inside leading to differential dimensional changes that are restrained by the adjacent volume elements within the piece, creating stress. [3 - 6]

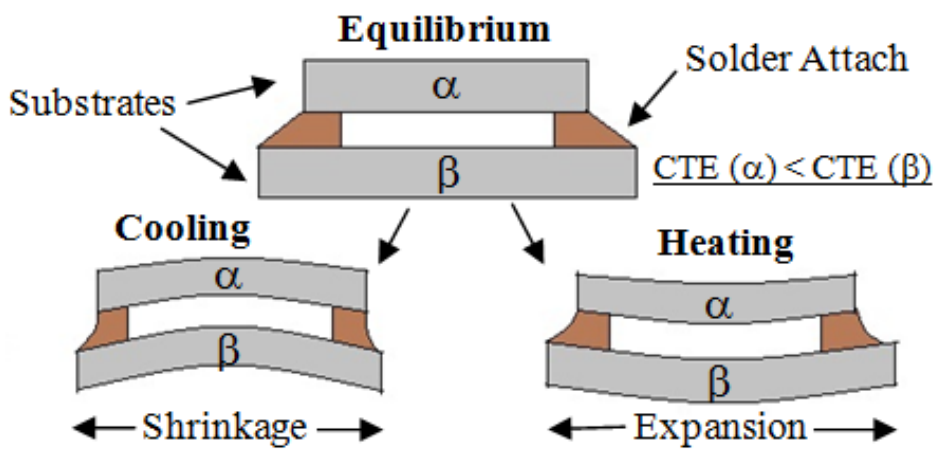

Figure 2.1: Effect of Cooling and Heating on Thermo-mechanical Stress Generation

Similarly, as shown in Figure 2.1 above, when there are two substrates with different coefficient of thermal expansions $\alpha$ and $\beta$ attached using with the help of solder, there will be a difference in the expansion of the two materials when they are heated or cooled, which is restrained by the other material creating stress. At room 
temperature or equilibrium position, there is no bending observed in the two substrates. However when a system in which C.T.E. $(\alpha)<$ C.T.E $(\beta)$, is subjected to heating, the bottom substrate undergoes greater expansion leading to a concave bowing from above and with cooling there is greater shrinkage observed in the bottom substrate, leading to a convex bowing from above.

\subsubsection{Thermo-mechanical Failure Mechanisms}

An electronic package consists of a number of material systems operating under electrical, thermal and mechanical loading conditions. [20] Many of these material systems are organic in nature, having non-linear properties, which are sensitive to processing and use conditions.

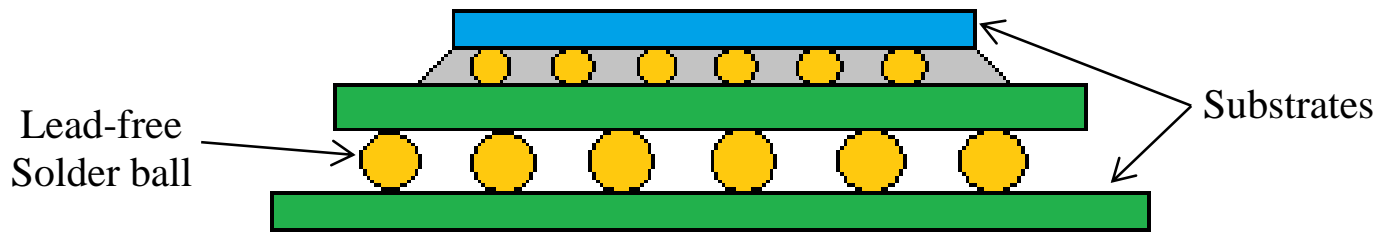

Figure 2.2: Schematic view of Flip Chip Ball Grid Array (FC PBGA), [20]

The above illustration demonstrates such a packaging system. [20] In such a flip chip plastic ball grid array (FC PBGA), a silicon die (device) is attached on a laminate substrate (glass fiber/epoxy) by small solder joints. In order to enhance the reliability of the solder joints, they are encapsulated by an underfill material. The underfill process completes the component-level packaging. The next level of packaging includes their connection to the printed wiring board by an array of relatively larger solder balls, which is generally termed as Ball Grid Array (BGA) solder balls. Some of the common failure mechanism observed in such a system 
include underfill delamination, solder fatigue failure, substrate fracture, interconnection failure, and die cracking.

The major driving force for occurrence of all of these failures at different levels in this package is the difference in coefficient of thermal expansion (C.T.E.) of the different materials used in this package. For example, the silicon device has a C.T.E. of $2.8 \mathrm{ppm} /{ }^{\circ} \mathrm{C}$ and that of laminate substrate is approximately $18 \mathrm{ppm} /{ }^{\circ} \mathrm{C}$, hence, there is a large difference in C.T.E. of these materials. This leads to significant thermal stress generation in the package when they are subjected to thermal cycles during operation of the device. As a consequence of this thermal stress, the associated failure mechanism is die cracking. In most cases, the crack initiates at the die edge and propagates towards the die active surface. The device functionality is affected when the crack reaches the active surface, which ultimately leads to failure of the component.

\subsubsection{Stress and Strain Analyses}

To predict the stress and strain distributions in a package under the given environmental and loading conditions, the analyses technique could be analytical, numerical or experimental in nature. [3 - 6] The theory of analytical methods can be divided into two basic approaches, namely, structural mechanics or continuum mechanics method. With the theory of structural mechanics, one considers various parts of the package as beams, plates and shells, etc. This approach is comparatively simpler in nature, which is the main advantage. Often times, it is easier to obtain analytical solutions. However, this technique has a disadvantage, which is that a lot of assumptions have to be made. [3 - 6] As a result, it gets difficult to capture all the 
characteristics of a stress field. On the other hand, a continuum mechanics approach considers the package as a three-dimensional structure. The advantage of a threedimensional analysis is the accuracy in results obtained. However, the solutions are generally lengthy and complicated in nature, which will require comparatively large time for obtaining solution.

Numerical methods are prone to be a better approach for stress and strain analysis. Finite Element Method (FEM), one of the numerical methods, is a very robust and effective tool for computing stress distribution in a package under various loading and environmental conditions. In this method, we assume a piecewise continuous function for solving a given problem and obtaining the parameters of the functions in a manner that reduces the error in solution. The underlying basis of FEM is the discretization of component under consideration into smaller elements. Also, this method assumes that the stresses on each component or finite element are constant or related by a linear function. The elements are assembled together to form a system of algebraic equations through overall compatibility and equilibrium requirements, with stresses or displacements on each element as the unknowns. The resulting system of equations is large for geometries with 3-D model and materials with non-linear properties as the analysis input. The number of equations generated contributes to the time taken for solution of a problem. Often a problem with 3-D geometry is reduced to one with a 2-D model, if possible. [3 -6] This will help in reduction of the number of equations generated and consequently, the solution time. 


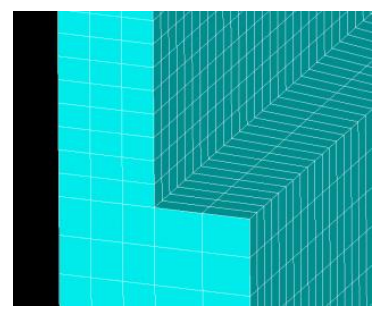

Figure 2.3: FEM Mesh Generation Example (1um x 1um x 1um)

As shown in figure above, a 3-D CAD geometry was imported to FEA software, and a mapped sweep mesh was generated to solve the thermo-mechanical problem. As it can be inferred from the figure, the geometry replicates a micro-fin used for dissipating heat from a device using a two-phase fluid. [3 - 6] The inputs to the FE model include thermal and structural boundary conditions with use of the appropriate material properties. In this thermal management problem, the inputs from thermal side included heat flux application at the die level and convection heat transfer coefficient application on the micro-fin surface. From a structural standpoint, the boundary conditions mainly include the displacement constraints and symmetry boundary conditions on respective surfaces. It is important to understand that it's easier to solve a complicated problem by considering a unit cell which replicates the whole system. In the present scenario, as shown above, the system with a large number of micro-fins is reduced down to a simpler unit system by considering a single micro-fin base geometry. One of the other things to keep in mind while solving an FEA problem is the method of mesh generation, meaning the use of correct element type. There are element types specifically designed to solve a particular physics problem of interest. For a coupled physics problem, example: a thermalmechanical system, we'll use an element that would have capability to solve both thermal and structural problem. Alternatively, an element type designed specifically 
for solving the thermal problem will be used first, which is ideally followed by use of an equivalent element capable of solving the structural problem. Additionally, a coupled physics problem will involve transfer of loads from one analysis to another along with use of proper boundary condition. [3]

\subsubsection{Failure Criteria and Predictions}

An electronics package consists of numerous components, which are subjected to complex loading conditions and consequently, the stress generation is complex in nature with stresses at a given point in the material often occurring in more than one direction. [3 - 6] If the magnitude of stresses is sufficiently severe, the stresses can act together to cause failure in the material. Selection of appropriate failure criterion is important to predict the safe limits for use of a material under combined stress condition. As explained previously, a failure criterion is a theory, which predicts when a certain combination of stresses will act together to cause a failure in the material. Mathematically, a failure criterion can be explained as follows,

$$
\bar{\sigma}=f\left(\sigma_{1}, \sigma_{2}, \sigma_{3}\right)=\sigma_{c} \text { at failure }
$$

where, $\bar{\sigma}$ is called the effective stress, $f\left(\sigma_{1}, \sigma_{2}, \sigma_{3}\right)$ is a given function which relates to the principal stresses: $\sigma_{1}, \sigma_{2}, \sigma_{3}$ and $\sigma_{c}$ is a material parameter. If the state of stress at a given point is expressed as following,

$$
\bar{\sigma}=f\left(\sigma_{1}, \sigma_{2}, \sigma_{3}\right)>\sigma_{c}
$$

then, one may predict that the component will undergo failure. The nature and type of failure criterion can be expressed specifically by a combination of the function, $f\left(\sigma_{1}, \sigma_{2}, \sigma_{3}\right)$. [3 - 6] Three commonly used failure criteria based on the material of application include as per the following table. 
Table 2.1: Selection of Failure Theory Based on Type of Material

\begin{tabular}{|c|c|c|c|}
\hline Name & $\boldsymbol{f}\left(\sigma_{1}, \sigma_{2}, \sigma_{3}\right)$ & $\sigma_{\boldsymbol{c}}$ & $\begin{array}{c}\text { Material } \\
\text { Type }\end{array}$ \\
\hline $\begin{array}{c}\text { Max. } \\
\text { Principal } \\
\text { Stress } \\
\text { theory }\end{array}$ & $\bar{\sigma} \max \left\{\left|\sigma_{1}\right|, \sigma_{2}\left|, \sigma_{3}\right|\right\}$ & $\begin{array}{c}\text { Uniaxial } \\
\text { yield } \\
\text { strength }\end{array}$ & Brittle \\
\hline $\begin{array}{c}\text { Von Mises } \\
\text { Stress } \\
\text { theory }\end{array}$ & $\bar{\sigma}$ & $\begin{array}{c}\text { Uniaxial } \\
\text { yield } \\
\text { strength }\end{array}$ & Ductile \\
\hline $\begin{array}{c}\text { Tresca } \\
\text { failure } \\
\text { criterion }\end{array}$ & $\bar{\sigma} \sqrt{\left(\sigma_{1}-\sigma_{2}\right)^{2}+\left(\sigma_{1}-\sigma_{2}\right)^{2}+\left(\sigma_{1}-\sigma_{2}\right)^{2}}$ & $\begin{array}{c}\text { Uniaxial } \\
\text { yield } \\
\text { strength }\end{array}$ & Ductile \\
\hline
\end{tabular}

If there are sharp flaws like cracks and notches present in the component, localized stresses can become significantly higher than nominal stresses due to stress concentration effect. For modeling failure and understanding the underlying physics, one should adopt the fracture mechanics approach to capture the localized stress gradient, which will ultimately lead to failure of the device. The theory of fracture mechanics is based on the assumption that the strength of stress fields is usually controlled by a single parameter. [3 - 6]

\subsection{Physics of Failure - PoF based Reliability Prediction Models}

\subsubsection{Weibull Statistical Distribution}

The Weibull distribution is a continuous probability distribution, named after the Swedish scientist, Waloddi Weibull. $[16,17]$ One of the main advantages of using a Weibull distribution is the fact it has ability to capture the features of other distribution functions like exponential and the Rayleigh distributions based on the values of the shape parameter - $\beta$. The distribution converges or approximates other 
distributions under special or limiting conditions. Also, it can be used to represent increasing, constant and decreasing hazard rates.

The three parameter Weibull pdf (probability density function) is given by:

where,

$$
f(t)=\frac{\beta}{\eta}\left(\frac{t-\gamma}{\eta}\right)^{\beta-1} e^{-\left(\frac{t-\gamma}{\eta}\right)^{\beta}}
$$

$\eta=$ scale parameter

$\beta=$ shape parameter (or slope)

$\gamma=$ location parameter (or failure free life)

The two parameter Weibull pdf can be given by setting $\gamma=0$, as follows:

$$
f(t)=\frac{\beta}{\eta}\left(\frac{t}{\eta}\right)^{\beta-1} e^{-\left(\frac{t}{\eta}\right)^{\beta}}
$$

The mean time to failure (MTTF) of Weibull pdf is given by:

$$
\bar{T}=\gamma+\eta \cdot \Gamma\left(\frac{1}{\beta}+1\right)
$$

where, $\Gamma\left(\frac{1}{\beta}+1\right)$ is the gamma function evaluated at the value of $\left(\frac{1}{\beta}+1\right)$.

The Weibull reliability function can be given as follows:

$$
R(t)=1-e^{-\left(\frac{t-\gamma}{\eta}\right)}
$$

\subsubsection{Effect of the Shape Parameter - $\beta$}

The Weibull shape parameter $(\beta)$ is also known as the slope. The shape parameter $(\beta)$ has significant effect on the behavior of the distribution. $[16,17]$ Different values of this parameter will cause the distribution equations to reduce to those of other distributions. When $\beta=1$, the equation for three parameter Weibull reduces to 2 parameter, which is the case of the exponential distribution. As shown in 
the illustration below, the failure rates for corresponding values of $\beta$ are plotted and it has marked effect on the distribution achieved.

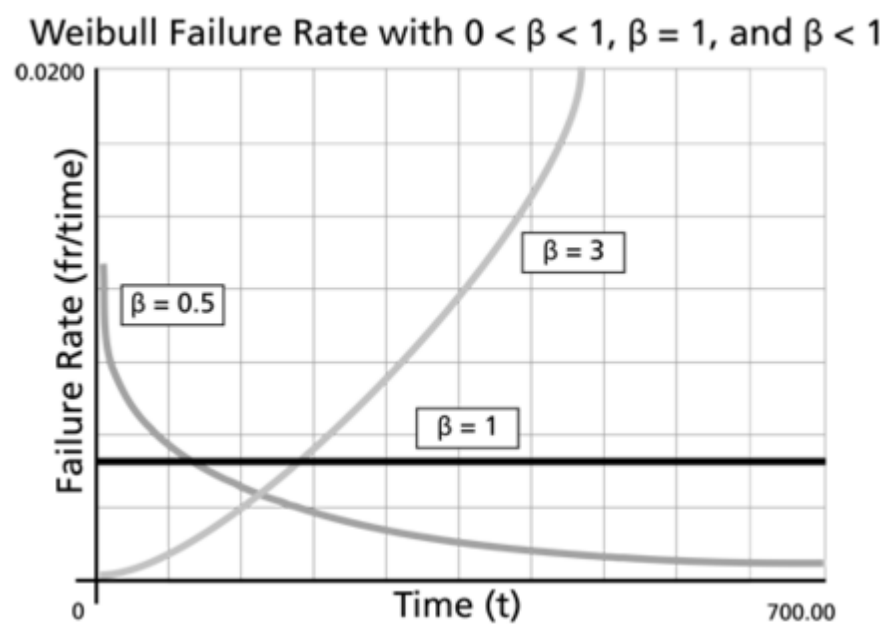

Figure 2.4: Effect of Shape Parameter on Weibull Failure Rate [54]

Population with $\beta<1$ exhibits a failure rate that decreases with time. For $\beta=$ 1 , the population has a constant failure rate and populations with $\beta>1$, have a failure rate which increases with time. As a result, one can model all three regions of the bath tub curve with the Weibull distribution and varying values of $\beta$.

The Weibull failure rate for the case of $0<\beta<1$ depicts the infant mortality stage of the bath tub curve, where the failure rate has a high value at time $t=0$ and thereafter decreases with time. For $\beta=1$, we can represent the failure rate of chancetype failures and the useful life of period failure rate of units. Lastly, we can represent the failure rate of units exhibiting wear-out type failures for values of $\beta>1$, as the failure rate increases with increase in time. $[16,17]$ 


\subsubsection{Effect of Location Parameter - $\gamma$}

\section{Effect of Location Parameter $\gamma$ on Weibull pdf}

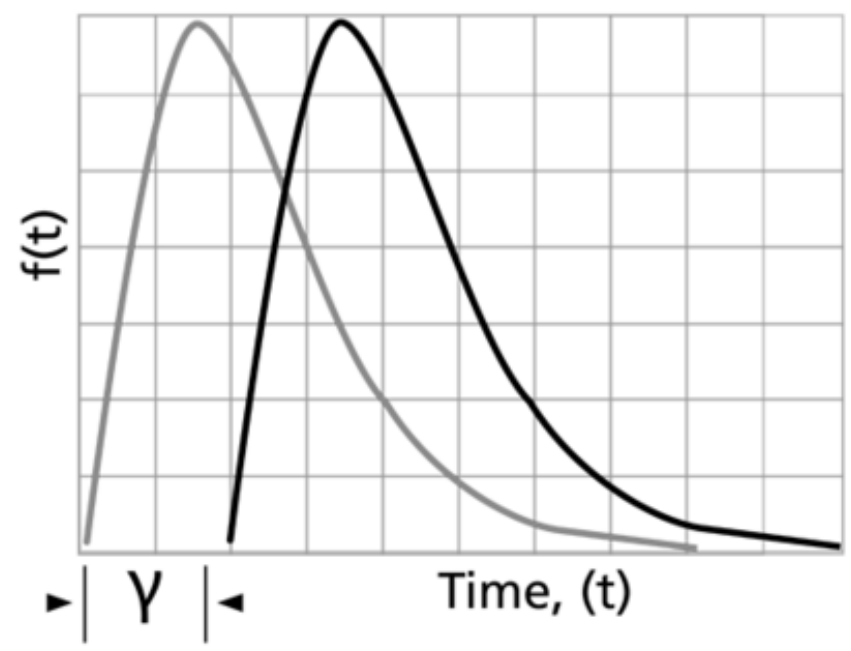

Figure 2.5: Effect of location parameter on Weibull pdf, [54]

The location parameter provides information regarding the time where the distribution starts. When $\gamma>0$, the distribution starts at the location $\gamma$ to the right of the origin and when $\gamma<0$, the distribution starts at the location $\gamma$ to the left of the origin. Hence, as the name implies, the location parameter $(\gamma)$ locates the distribution along the abscissa. The location parameter $(\gamma)$ provides an estimate of the earliest time-to-failure of units under consideration. The failure free operating period of the units is the life period from 0 to $\gamma$. One of the important conclusions to be drawn from the use of location parameter $(\gamma)$ is that, if $\gamma<0$, it implies that the failures have occurred prior to the beginning of the test. This essentially means that there might be some defects produced in the material during production, storage, transportation or prior to the start of actual usage of the product. $\gamma$ has a unit of time, which could be in hours, miles, cycles, etc. $[16,17]$ 
A failure free operating period is a period of time during which no failures occur. A location parameter, gamma $(\gamma)$ greater than 0 , can be interpreted as a failurefree metric. It should not be confused with a maintenance free operating period, which is a period of time during which a system is reliable without maintenance.

\subsection{Fatigue Life Prediction Physics of Failure - PoF based Reliability Models}

\subsubsection{Coffin Manson Model}

The reliability of solder joints is an important concern in the packaging of electronics. [21] Solder joints are known to fail in fatigue by cyclic strain induced by fluctuations in temperature under operational and environmental loading conditions. The cyclic strains are produced by the global difference in CTE (Coefficient of Thermal Expansion) between the surfaces to be interconnected (e.g. substrates, chip, device) and the local difference in CTE between the surfaces and the solder. Morrow proposed a generalized fatigue damage law for metals, which was based on cumulative visco-plastic strain energy density. The Coffin-Manson plastic strainfatigue life relationship has been derived from Morrow's model. One of the drawbacks with use of Coffin Manson model is that it fails to capture the relationship among the parameters like frequency, temperature, and lead stiffness, which has limited its applicability to various problems related to failure/reliability of solder joints. [21]

On the basis of experimental fatigue data presented by R.N. Wild for 63Sn37Pb solder joints used for interconnecting leadless devices on printed wiring boards, Engelmaier proposed a failure model, [22] which was later extended to leaded 
parts. Engelmaier's model integrated parameters like cyclic frequency, solder and substrate temperature. Hence, his model addressed the limitations of original CoffinManson model and proves to be a more versatile model for life-cycle prediction of solder joints. That being said, his model has several deficiencies and assumptions associated with it. One of the drawbacks associated with his model is that it's applicable only to certain package types. It did not account well for solder joint geometry and couldn't handle the creep of a solder joint. Engelmaier worked on the deficiencies of his model but couldn't eliminate all the drawbacks. As a result of which, other research scientists suggested various models, including the creep-strainbased models, energy-based models, damage-based fatigue models [22] and others to address the limitations of Engelmaier's model. Even with its deficiencies and limitations, Engelmaier's model is the most widely used for prediction of solder joint reliability.

\subsubsection{Relationship for Coffin-Manson Equation}

The original Coffin-Manson equation only includes the shear strain $(\Delta \gamma)$ as the powered term. But the modified Coffin Manson relationship gives the fatigue life of solder joints based on the induced cyclic shear strain as per the following relationship $[21,22]$.

$$
N_{f}=\left(\frac{A}{\Delta \gamma}\right)^{m} f^{n} e^{-\beta / k T_{\max }}
$$

where, $N_{f}$ is the number of cycles to failure, A is a material constant, $\Delta \gamma$ is the cyclic plastic shear strain of a solder joint, $f$ is the cyclic frequency, $T_{\max }$ is the maximum temperature during the cycle, $k$ is the Boltzmann's constant, $n$ is an empirical 
constant, and $\beta$ may be thought of as an empirical activation energy. This version does incorporate a frequency and temperature term. The value of $\mathrm{m}$ varies from 1.89 to 2.5 , as per literature [22], and inversely relates the cycles-to-failure to the shear strain.

\subsubsection{Engelmaier's Model}

The equation relating the number of cycles to failure to the plastic strain was developed by Engelmaier [22, 23] in the early 1980's and is given as following.

$$
N_{f}=0.5\left(\frac{\Delta \gamma}{2 \varepsilon_{f}}\right)^{\left(\frac{1}{c}\right)}
$$

where, $N_{f}$ is the mean cycles to failure, $\varepsilon_{f}^{\prime}$ is the fatigue ductility coefficient $\left(2 \varepsilon_{f}^{\prime} \approx\right.$ 0.65 for eutectic and $60 \mathrm{wt} . \% \mathrm{Sn}-40 \mathrm{wt} . \% \mathrm{~Pb}$ solder), and $\mathrm{c}$ is the fatigue ductility exponent.

The fatigue ductility exponent for near eutectic tin-lead solder is given as follows:

$$
c=-0.442-6 * 10^{-4} \bar{T}_{s}+1.74 * 10^{-2} \ln (1+f)
$$

In the above equation, $\bar{T}_{S}$ is the mean cyclic solder joint temperature, which is the average of the maximum and minimum temperatures, ${ }^{\circ} \mathrm{C}$ and $f$ is the cyclic frequency $(1 \leq f \leq 1000$ cycles/day). The model given above is the first version formulated for leadless chip carriers. In the above equation, $\Delta \gamma$ is the cyclic shear strain, [41] which can be computed using the equation below for corner solder joint of height $\mathrm{h}$ in a square ceramic chip carrier (CCC) with length $\mathrm{L}$. The equation for cyclic shear strain is given as follows. 


$$
\Delta \gamma=\frac{L}{\sqrt{2} h} \Delta(\alpha \Delta T)_{s s}
$$

where, $\Delta(\alpha \Delta T)_{s s}$ is the in-plane steady state thermal expansion mismatch, which is given using the following equation.

or

$$
\Delta(\alpha \Delta T)_{s s}=\left(\alpha_{c}-\alpha_{s}\right)\left(T_{c}-T_{o}\right)+\alpha_{s}\left(T_{c}-T_{s}\right), \text { powered }
$$

$$
\Delta(\alpha \Delta T)_{s s}=\left(\alpha_{c}-\alpha_{s}\right)\left(T_{c}-T_{o}\right), \text { unpowered }
$$

$T_{c}$ and $T_{S}$ are the steady state operating temperatures for the component and substrate, respectively, and $T_{o}$ is the power off, steady-state temperature. [22]

As compared to the original Coffin Manson model, the one given by Engelmaier, as stated above, incorporated parameters for stress relaxation effects, like cyclic frequency and average solder joint temperature. As it can be inferred from the equation, this model neglected the effect of board warpage and transient strains. The empirical constants of the model were based on the correlation of isothermal shear fatigue data given by Wild. The model also assumed that the strain distribution in the joint geometry is uniform. The joint geometry assumed is a cylindrical solder joint with fillets on both sides which prevent strain concentrations and offset the effects of intermetallic embrittlement. One of the other statements he made regarding the applicability of this model was that for solder joints with larger solder joint height, the model does not capture the deviations from the condition of pure shear as the joint is subjected to tensile and compressive stresses mainly at the interfaces due to joint distortions. Also, Engelmaier assumed a stiff substrate. However, in actual application condition, we often observe a cyclic warpage in substrate due to the effects of thermal expansion mismatch and temperature gradient, unless the substrate is stiffened. [22] 
Engelmaier updated the drawbacks in his initial model later. He modified the cyclic frequency term in the model above to focus on the half cycle dwell time at the maximum temperature. He also modified the relationship with a factor, F, to take into account effects like cyclic warpage, non-ideal solder joint geometry, and brittle intermetallic compounds.. The modified equations are as follows.

$$
\begin{gathered}
c=-0.442-6 * 10^{-4} \bar{T}_{S}+1.74 * 10^{-2} \ln \left(1+\left(\frac{360}{t_{D}}\right)\right) \\
\Delta \gamma=F \frac{d}{2 h} \Delta(\alpha \Delta T)
\end{gathered}
$$

where, $\mathrm{F}$ is the empirical factor accounting for second order effects and $\mathrm{d}$ is the longest distance on the component between solder joints. [22, 23]

\subsection{Deficiencies in Engelmaier's Model}

There are various shortcomings in Engelmaier's model which have been partially addressed in later research. Engelmaier identified six caveats in his model (includes assumptions and areas of concern), which are namely, 1) solder quality, 2) large temperature excursions, 3) high frequency/low temperature, 4) local expansion mismatch, 5) lead-solder CTE mismatch, and 6) very stiff leads/large expansion mismatches. [22, 23]

\subsubsection{Shortcomings in the Fatigue Model}

Research scientists Barker and Kaspari in their works [22] reviewed the deficiencies in Engelmaier's fatigue model. They found that the non-ideal factor F, which is assumed to be a constant, is a function of thermal cycle temperature ranges. Additionally, they found that his model is sensitive to the solder joint height. Also, 
the model doesn't take into account the parameters that control the soldering process. Salmela [22] found that his model doesn't take into consideration the structural details of the component. As an example, the model doesn't differentiate between the peripheral and area type package configurations. Scholand et. al. [22] noticed that Engelmaier's model accounts only for shear plastic deformation because the Coffin Manson plot for solder is completely dominated by plastic deformation. It fails to account the elastic strain component.

\subsubsection{Solder Joint Height and Quality}

Quality of solder is one of the factors which the industry specific standards of IPC-SM785 and IPC-D-279 state was not incorporated by Engelmaier in his model.. . Also, Engelmaier, himself, stated that his model is not suitable for solder joints with heights less than $50 \mathrm{um}$ to $75 \mathrm{um}$. The rationale given was that for solder joints so thin, the solder gap is composed primarily not of bulk solder but of the intermetallic compounds (IMCs) formed from the solder reaction with the base metal surfaces. As a consequence, the equations for life prediction given by Engelmaier are not applicable. The IMCs don't creep at the prevailing temperatures and are typically more brittle and stronger in nature. [22]

\subsubsection{Local Coefficient of Thermal Expansion - CTE Mismatches}

One of the major drawbacks of Engelmaier's model is that it fails to consider the local thermal expansion mismatch between the component terminals and solder as well as the solder and the substrate or PWB bonding pad. He stated that for applications where the global thermal expansion mismatch effect was small (e.g., 
silicon on silicon, ceramic on ceramic), the primary cause of fatigue damage was local thermal expansion mismatch. [22]

\subsubsection{Temperature Range}

Engelmaier's model also seems to be applicable only for a particular temperature range. $[22,23]$ For temperatures below $-20{ }^{\circ} \mathrm{C}$, the stress relaxation and creep in solder joint are incomplete in nature and his model will not hold true for such conditions. At higher temperatures, more complex methods can be applied to study the solder joint life. An energy partitioning approach can be applied to study the effect of creep-fatigue damage accumulation on solder joint.

In research by Evans et. al. [22], it is stated that Engelmaier's model is suitable for a temperature range of $0+/-100{ }^{\circ} \mathrm{C}$ and to a cycle with a symmetrical hold time allowing significant stress relaxation.

\subsubsection{Cycle Frequency}

As per the industry standard IPC-SM-785 [46] and Engelmaier's own statement, Engelmaier's model will not be appropriate to apply for conditions where the change in strain is very rapid (i.e., frequency $\mathrm{f}>0.5 \mathrm{~Hz}$ or $t_{D}<1 \mathrm{~s}$ ), for which stress relaxation and creep in the solder joint is not a dominant mechanism. [22]

\subsection{Engelmaier's Model for Lead Free Solders}

The failure model proposed by Engelmaier for $\mathrm{SnPb}$ eutectic solders has been widely adopted and used in the industry. However, since $2006, \mathrm{SnPb}$ solders have been replaced by lead-free solders, in order to comply with RoHS legislation. This has created a need for a similar reliability prediction model for such materials. [22, 
23] In a study conducted by CALCE (Center for Advanced Life Cycle Engineering), University of Maryland at College Park, the effect of dwell time and mean temperature on lead free solders $(\mathrm{Sn} 3.9 \mathrm{Ag} 0.7 \mathrm{Cu}$ and $\mathrm{Sn} 3.5 \mathrm{Ag})$ was evaluated using leadless ceramic chip carrier (LCCC) assemblies. Leaded components (TQFPs and TSOPs) were also used. $[22,23]$ The test data was then fitted to the fatigue model form proposed by Engelmaier.

A model was proposed $[22,23]$ for lead free solders in the article "CreepFatigue Model for SAC 405/305 Solder Joint Reliability Estimation”. This study was based on accelerated test results, fitted to the Engelmaier model with four parameters modified by regression analysis. After running an analysis for leadless components, the predicted results were found to be within a factor of two of each other, which was well within the expected experimental variation.

Based on the data obtained from the CALCE model curve for accelerated test results, Engelmaier proposed a failure model for SAC405 and SAC305 solder joints as follows.

$$
\begin{gathered}
N_{f}(50 \%)=\frac{1}{2}\left[\frac{0.480}{\Delta D}\right]^{m} \\
\frac{1}{m}=0.390+9.3 * 10^{-4} \bar{T}_{S J}-1.92 * 10^{-2} \ln \left(1+\frac{100}{t_{D}}\right) \\
\frac{1}{m}=c_{0}+c_{1} \bar{T}_{S J}+\ln \left(1+\frac{t_{0}}{t_{D}}\right)
\end{gathered}
$$

Engelmaier recommended that this model should be used for Sn-based lead free solder in place of the older Engelmaier's model. The values of the parameters are shown in the table as follows. [22, 23] 
Table 2.2 Engelmaier's Constants for Various Solders, [22]

\begin{tabular}{|c|c|c|c|c|c|}
\hline Solder & $\boldsymbol{\varepsilon}_{\mathbf{f}}{ }^{\prime}$ & $\mathbf{c}_{\mathbf{0}}$ & $\mathbf{c}_{\mathbf{1}}$ & $\mathbf{c}_{\mathbf{2}}$ & $\mathbf{t}_{\mathbf{0}}$ \\
\hline SnPb & 0.325 & 0.442 & $6.00 \mathrm{e}-4$ & $-1.74 \mathrm{e}-2$ & 360 \\
\hline SAC405/305 & 0.240 & 0.390 & $9.30 \mathrm{e}-4$ & $-1.92 \mathrm{e}-2$ & 100 \\
\hline SAC205 & $<0.240$ & not known & not known & not known & 100 \\
\hline SAC105 & $<0.240$ & not known & not known & not known & 100 \\
\hline SnAg & $<0.325$ & not known & not known & not known & -60 \\
\hline
\end{tabular}

\subsection{Yielding Phenomenon for Ductile and Brittle Materials}

\subsubsection{Mechanical Properties of Materials}

The mechanical behavior of a material reflects its response or deformation in relation to an applied load or force. Some of the important mechanical properties from a design perspective include: stiffness, strength, hardness, ductility and toughness. [34]

\subsubsection{Phenomenon of Yielding}

When a structure is designed, one of the important factors for consideration is the fact that the structural material should only undergo elastic deformation when it is subjected to a given loading condition. A structure which has plastically deformed will experience a permanent change in its shape and may not be able to perform its intended function after this permanent deformation. Hence, it is utmost important to understand the stress level at which this given structural material will undergo plastic deformation or the point at which the phenomenon of yielding occurs. [33, 34]

In metals, the phenomenon of yielding occurs when we see a gradual transit in its mechanical behavior from elastic regime to plastic regime under an imposed load. The point of transition is generally referred to as the yielding point of the material. Hence, one can understand the onset of plastic deformation as the beginning of 
yielding in the material. It is often difficult to measure the position of the yielding point on a stress-strain curve; hence, a general rule of thumb is to consider a straight line that is constructed parallel to the elastic portion of the stress-strain curve at some specified strain offset, usually taken as 0.002 . The stress corresponding to the intersection of this offset line on the stress-strain curve is referred to as the yield strength $\left(\sigma_{\mathrm{y}}\right)$ of the material under consideration. The units of yield strength are MPa or psi. For materials having a non-linear elastic region, the use of strain offset method is not possible. General practice adopted for such a case is to define the yield strength as the stress required to produce some amount of strain. [33, 34]

After onset of yielding, the stress necessary to cause plastic deformation in the material increases to a maximum point and then decreases until the material undergoes fracture. The tensile strength is the stress at the maximum on the engineering stress-strain curve. Alternatively, the tensile strength gives us an idea about the maximum stress that a structure can sustain under tensile loading. Also, if this stress is applied and maintained, the structure will undergo fracture. At this maximum stress value, a neck begins to form at some point and all subsequent deformation is focused near this neck region. This phenomenon is termed as necking and it ultimately leads to fracture in the material. The fracture strength corresponds to the stress at fracture. $[33,34]$

\subsubsection{Cauchy Stress Tensor}

With the help of continuum mechanics, we can define the Cauchy stress tensor $\sigma$ (true stress tensor), which gives the state of stress (with nine components $\sigma_{\mathrm{ij}}$ ) at a point inside a material in the deformed placement or configuration. [24, 26] The 
tensor relates a unit-length direction vector $\mathrm{n}$ to the stress vector $\mathrm{T}^{(\mathrm{n})}$ across an imaginary surface perpendicular to $\mathrm{n}$. It can be defined as follows.

$$
T^{(n)}=n . \sigma \quad T_{j}^{(n)}=\sigma_{i j} n_{i}
$$

where,

$$
\sigma=\left[\begin{array}{lll}
\sigma_{11} & \sigma_{12} & \sigma_{13} \\
\sigma_{21} & \sigma_{22} & \sigma_{23} \\
\sigma_{31} & \sigma_{32} & \sigma_{33}
\end{array}\right]=\left[\begin{array}{lll}
\sigma_{x x} & \sigma_{x y} & \sigma_{x z} \\
\sigma_{y x} & \sigma_{y y} & \sigma_{y z} \\
\sigma_{z x} & \sigma_{z y} & \sigma_{z z}
\end{array}\right]=\left[\begin{array}{ccc}
\sigma_{x} & \tau_{x y} & \tau_{x z} \\
\tau_{y x} & \sigma_{y} & \tau_{y z} \\
\tau_{z x} & \tau_{z y} & \sigma_{z}
\end{array}\right]
$$

The Cauchy stress tensor is applicable under change in the system of coordinates since it obeys the tensor transformation. Mohr's circle for stress is the graphical representation of this transformation law. $[24,26]$

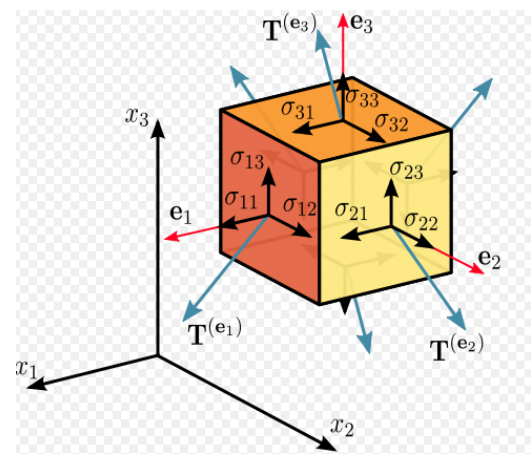

Figure 2.6: Components of Stress in three dimensions

For material bodies experiencing small deformations, the Cauchy stress tensor is applicable for their stress analysis. It is a widely used concept in the linear theory of elasticity. [24] According to continuum mechanics, a deformation in a solid body is said to be small when the displacements of the material's particles are assumed to be infinitesimally small with respect to the relevant dimension of the body, so that its geometry and the constitutive properties of the material (such as stiffness and density) at each point of space can be assumed to be unchanged by the deformation. 
As per the theory of conservation of linear momentum, if a continuum body is assumed to be in static equilibrium, then it can be proved that the components of the Cauchy stress tensor in every material point in the body satisfy the equilibrium equations. Also, according to the principle of conservation of angular momentum, equilibrium requires that the summation of the moments with respect to any arbitrary point is zero, and hence, we can conclude that the stress tensor is symmetric. Thus, the original nine components reduce down to six independent stress components.

The three principal stresses are the three eigenvalues of the stress tensor. They are invariants associated with the stress tensor and their values do not depend upon the coordinate system chosen, or the area element upon which the stress tensor operates.

\subsubsection{Principal Stresses and Stress Invariants}

There are three planes at any point inside a stressed body, called the principal planes, with normal vectors $n$ referred to as the principal directions. [24] At this point the corresponding stress vector is perpendicular to the plane, i.e. parallel or in the same direction as the normal vector $\mathrm{n}$. At this point there is no normal shear stress $\tau_{\mathrm{n}}$. Principal stresses are the three stresses normal to theses principal planes.

As mentioned previously, the components $\sigma_{\mathrm{ij}}$ of the stress tensor depend on the orientation of the coordinate system at the point under consideration. The stress tensor is independent of the coordinate system chosen to represent it. With every tensor, there are invariants associated with it which are also independent of the coordinate system. Example: a vector is a tensor of order one, which has three components in three dimensional space. The value of the components depend on the 
coordinate system chosen to represent the vector, however, the magnitude of the vector is a physical quantity independent of the Cartesian coordinate system chosen to represent the vector. Similarly, a second order tensor (stress and strain tensor) has three independent invariant quantities associated with it. Principal stresses of the stress tensor are one set of such invariants, which are the eigenvalues of the stress tensor. Principal directions or eigenvectors are the direction vectors of this second order tensor. [24]

A stress vector parallel to the unit vector $n$ is given by:

$$
T^{(n)}=\lambda n=\sigma_{n} n
$$

where, $\lambda$ is the constant of proportionality and in this case it corresponds to the magnitudes $\sigma_{\mathrm{n}}$ of the normal stress vectors or principal stresses.

The principal stresses can be combined to form the stress invariants, $\mathrm{I}_{1}, \mathrm{I}_{2}$ and $\mathrm{I}_{3}$. The first invariant is the trace of the stress tensor and third invariant is the determinant of the stress tensor. Thus, we can write the three invariants as follows.

$$
\begin{aligned}
& I_{1}=\sigma_{1}+\sigma_{2}+\sigma_{3} \\
& I_{2}=\sigma_{1} \sigma_{2}+\sigma_{2} \sigma_{3}+\sigma_{3} \sigma_{1} \\
& I_{3}=\sigma_{1} \sigma_{2} \sigma_{3}
\end{aligned}
$$

The principal stresses are often useful when considering the state of elastic medium at a particular point. They can be defined by the following relationship.

$$
\begin{gathered}
\sigma_{1} \sigma_{2}=\frac{\sigma_{x}+\sigma_{y}}{2} \pm \sqrt{\left(\frac{\sigma_{x}-\sigma_{y}}{2}\right)^{2}+\tau^{2} x y} \\
\tau_{\max }, \tau_{\min }= \pm \sqrt{\left(\frac{\sigma_{x}-\sigma_{y}}{2}\right)^{2}+\tau_{x y}^{2}}
\end{gathered}
$$


The maximum shear stress or maximum principal shear stress is equal to onehalf the difference between the largest and smallest principal stresses, and acts on the plane that bisects the angle between the directions of the largest and smallest principal stresses. This implies that the plane of maximum shear stress is oriented $45^{\circ}$ from the principal stress planes. The maximum shear stress can be given by the following equation. [24]

$$
\tau_{\max }=\frac{1}{2}\left|\sigma_{\max }-\sigma_{\min }\right|
$$

When the stress tensor is non-zero, the normal stress component acting on the plane for the maximum shear stress is non-zero and it is equal to the following.

$$
\sigma_{n}=\frac{1}{2}\left(\sigma_{1}+\sigma_{3}\right)
$$

\subsubsection{Stress Deviator Tensor}

The stress tensor $\sigma_{i j}$ has two parts [24], a hydrostatic stress tensor (volumetric stress tensor) $-\pi \delta_{i j}$, which corresponds to the volume change in the stressed body and a deviatoric stress tensor $-\delta_{i j}$, which is related to the distortion in the body.

Accordingly, we can write the stress tensor as follows.

$$
\sigma_{i j}=\delta_{i j}+\pi \delta_{i j}
$$

where, $\pi$ represents the mean stress and is given as per the following relationship.

$$
\pi=\frac{\sigma_{k k}}{3}=\frac{\sigma_{11}+\sigma_{22}+\sigma_{33}}{3}=\frac{1}{3} I_{1}
$$

The deviatoric stress tensor can be obtained by subtracting the hydrostatic tensor from the Cauchy stress tensor. [24]

$$
s_{i j}=\sigma_{i j}-\frac{\sigma_{k k}}{3} \delta_{i j}
$$


Since the deviatoric stress tensor is a second order tensor, it has a set of invariants, which can be obtained by the same process as the invariants of the Cauchy stress tensor were obtained. The principal directions of the stress deviator tensor $s_{i j}$ are the same as the principal directions of the stress tensor $\left(\sigma_{i j}\right)$.

The first, second and third deviatoric stress invariants $\left(J_{1}, J_{2}\right.$ and $J_{3}$ resp. $)$ can be expressed as a function of the components of stress deviator tensor $\left(s_{i j}\right)$ or its principal values $s_{1}, s_{2}$ and $s_{3}$. They are written as follows. [24]

$J_{1}=s_{k k}=0$

$J_{2}=\frac{1}{2} s_{i j} s_{j i}=\frac{1}{6}\left[\left(\sigma_{1}-\sigma_{2}\right)^{2}+\left(\sigma_{2}-\sigma_{3}\right)^{2}+\left(\sigma_{3}-\sigma_{1}\right)^{2}=\frac{1}{3} I_{1}{ }^{2}-I_{2}\right.$ $J_{3}=\operatorname{det}\left(s_{i j}\right)=s_{1} s_{2} s_{3}=\frac{2}{27} I_{1}{ }^{3}-\frac{1}{3} I_{1} I_{2}+I_{3}$

Since $s_{k k}=0$, the stress deviatoric tensor is in a state of pure shear.

The equivalent stress or Von-Mises stress can be defined as following.

$$
\sigma_{e}=\sqrt{3 J_{2}}=\sqrt{\frac{1}{2}\left[\left(\sigma_{1}-\sigma_{2}\right)^{2}+\left(\sigma_{2}-\sigma_{3}\right)^{2}+\left(\sigma_{3}-\sigma_{1}\right)^{2}\right]}
$$

\subsubsection{Von Mises Yield Criterion}

As per the principle of Von Mises yield criterion, materials begin to yield when the second deviatoric stress invariant $J_{2}$ reaches a critical value. As a consequence of which, it is often known as the $J_{2}$ plasticity or $J_{2}$ flow theory. This theory is best applicable to ductile materials. [25]

The theory explains further that a ductile material is said to start yielding when its Von Mises stress reaches a critical value, known as the yield strength of the material $\left(\sigma_{y}\right)$. [25] Additionally, the Von Mises yield criterion is independent of the first stress invariant $I_{1}$. Hence, it can be applied to study plastic deformation in ductile 
materials since the onset of yield of these materials doesn't depend on the hydrostatic component of the stress tensor.

Mathematically, Von Mises yield criterion is expressed as: $J_{2}=k^{2}$ ( $\mathrm{k}$ is the yield stress of the material in pure shear) and $k=\frac{\sigma_{y}}{\sqrt{3}}$.

On substitution of $J_{2}$ in terms of the Cauchy stress tensor components, we get an equation of the yield surface in terms of a circular cylinder, whose intersection with the deviatoric plane is a circle with radius $\sqrt{2} K$ or $\sqrt{\frac{2}{3} \sigma_{y}}$. As a consequence of which, the yield condition is independent of the hydrostatic stress.

The equation is given as follows.

$$
\begin{gathered}
\sigma_{v}=\sigma_{y}=\sqrt{3 J_{2}} \\
\sigma_{v}{ }^{2}=3 J_{2}=3 k^{2}
\end{gathered}
$$

Substituting $J_{2}$ in terms of the Cauchy stress tensor components gives yield surface:

$$
\sigma_{v}^{2}=\frac{1}{2}\left[\left(\sigma_{11}-\sigma_{22}\right)^{2}+\left(\sigma_{22}-\sigma_{33}\right)^{2}+\left(\sigma_{33}-\sigma_{11}\right)^{2}+6\left(\sigma_{23}^{2}+\sigma_{31}^{2}+\sigma_{12}^{2}\right)\right.
$$

The following figure explains how we get the yield surface as a circular cylinder, and on intersection of this surface with deviatoric plane, a circle with radius $\sqrt{2} K$ is obtained. $[25,27]$

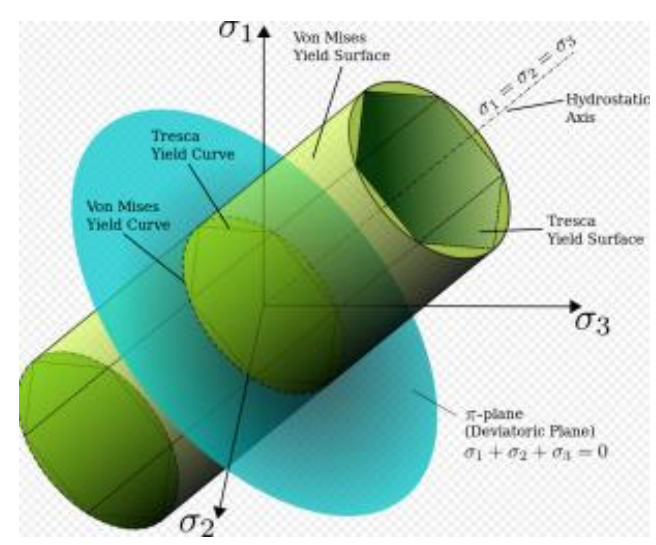

Figure 2.7: Von Mises Yield Surface in Principal Stress Coordinates 


\subsubsection{Maximum Principal Stress Theory}

As explained previously, the principal stresses are the stresses which act at an angle $\theta_{p}$ that defines the principal directions and where the only stresses are normal stresses. The following figure gives a better description about same. [24, 33]
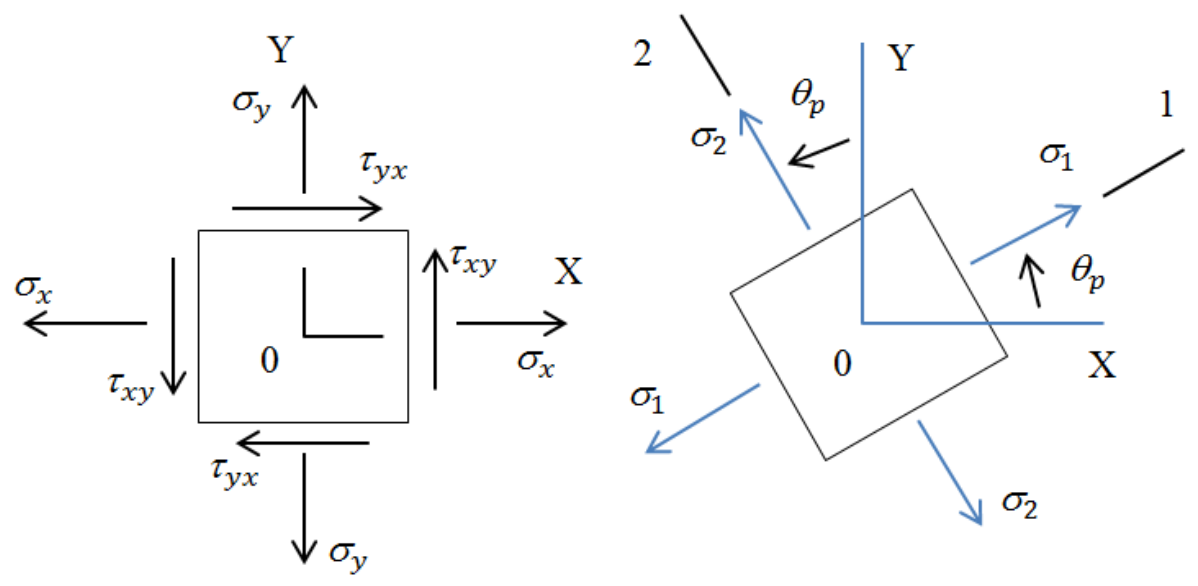

Figure 2.8: Transformation to Principal Directions

As per this theory, failure in a material occurs when the maximum principal stress reaches the fracture strength $\left(\sigma_{\mathrm{f}}\right)$. Hence, we need to find the maximum principal stress in the given material and compare the same with its tensile fracture strength $\left(\sigma_{\mathrm{f}}\right)$ to predict if the material will fail. This theory is best applicable to predict failure in brittle materials.

The following graph explains which yielding theory should be used to for predicting the failure in brittle and ductile materials. It shows the test done on a set of materials by different scientists (Lessels, Davis, Naghdi, Marin and Grassi). [33, 34] According to the following normalized principal stress plot, we see that brittle materials follow the maximum principal stress criterion and ductile materials like steel fall on the yield surface that observed by Von-Mises yield criterion. 


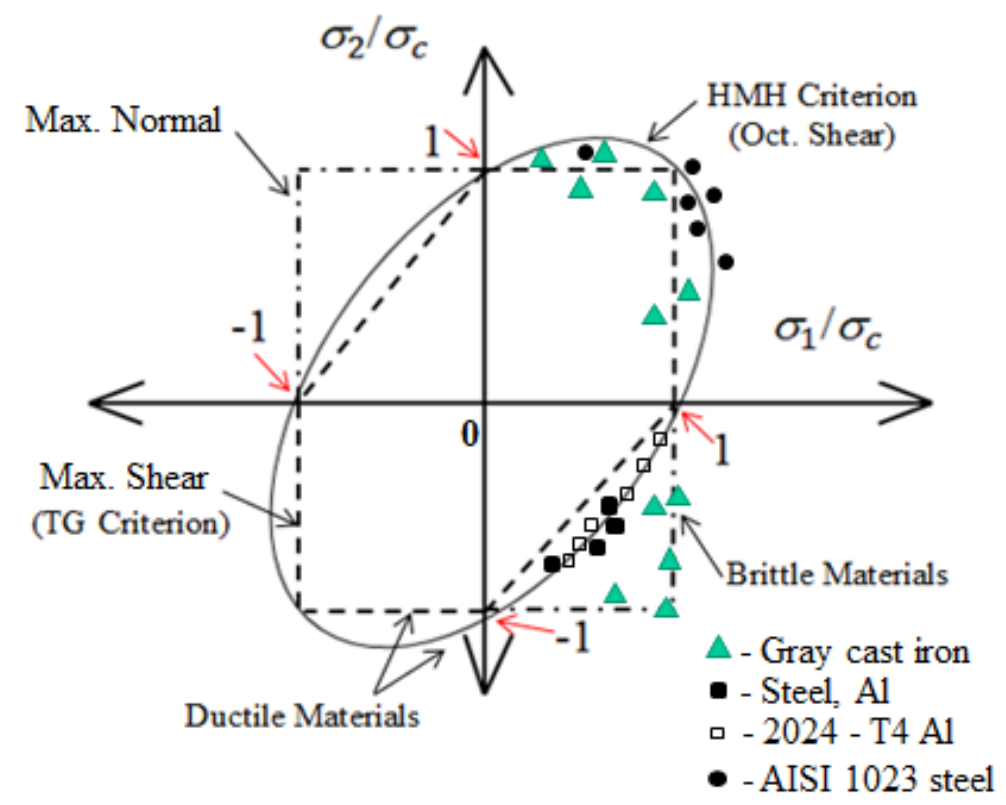

Figure 2.9: Yielding Phenomenon in Ductile and Brittle Materials, [33] 


\section{Chapter 3: TE Cooler Integration and Material Selection}

\subsection{Thermoelectric Mini-contact Based Cooling for Hotspot Heat Flux Removal}

This thesis focuses on the structural integrity of Mini-contact based embedded thermoelectric cooling. This integrated cooling system is designed to remove $5 \mathrm{k}$ $\mathrm{W} / \mathrm{cm}^{2}$ of heat flux from a $200 \mu \mathrm{m} \times 200 \mu \mathrm{m}$ hot spot on a HEMT power electronics device made in an epitaxial layer of $\mathrm{GaN}$ on a $\mathrm{SiC}$ base. This cooling technology is based on earlier research [35 - 37] on innovative spot cooling methods for high heat flux regions (e.g. q" $>1000 \mathrm{~W} / \mathrm{cm}^{2}$ ), including the use of Mini-contacts as shown in figure 3.1.
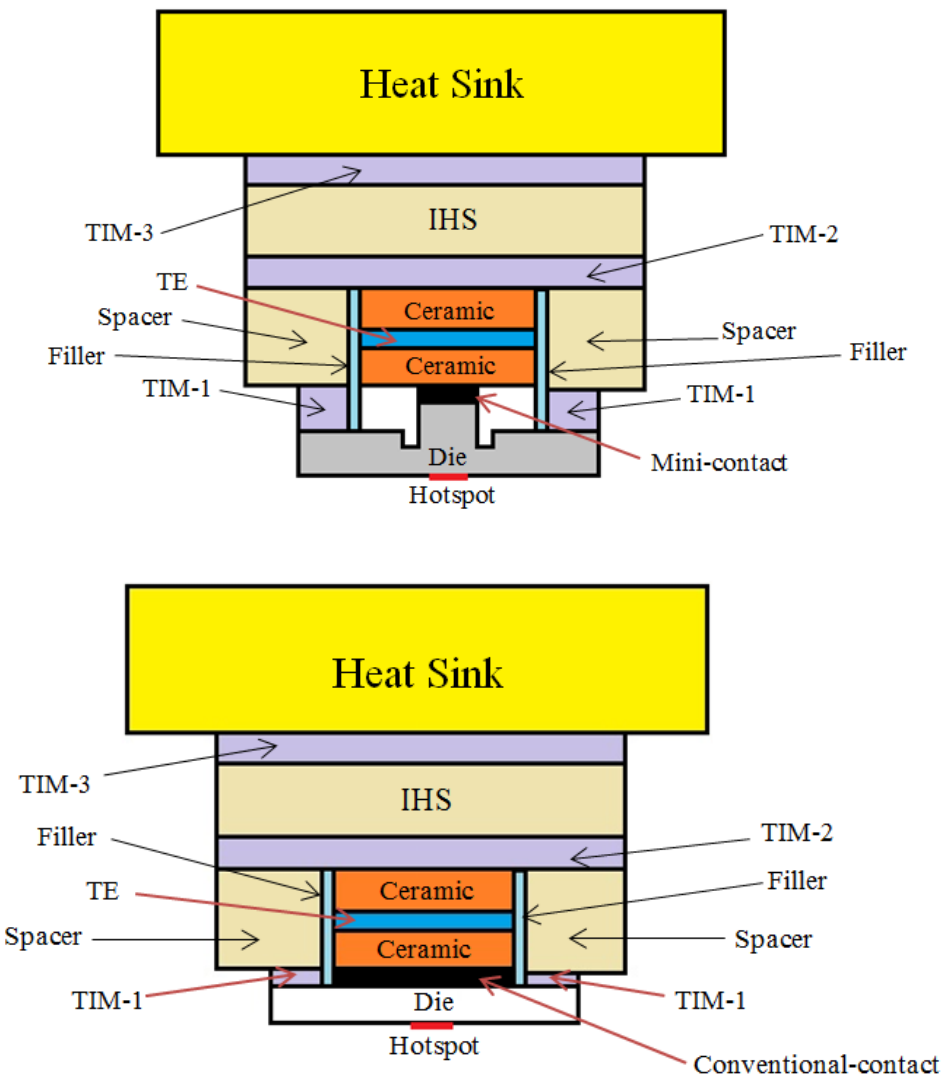

Figure 3.1: Schematic of TE Mini-contact and Conventional hot-spot cooling 
In the above research [35 - 37], Wang et al. reported that this novel cooling technique, which places a TE cooler on top of a Mini-contact that is integrated with a conventional integrated heat spreader (IHS) and heat sink, can provide cooling at the hot-spot $\left(1250 \mathrm{~W} / \mathrm{cm}^{2}, 400 \mu \mathrm{m} \times 400 \mu \mathrm{m}\right)$ about $19^{\circ} \mathrm{C}$ better than that achieved in a conventional package without TEC.

\subsubsection{Mini-contact based Cooling Methodology}

Thermal considerations limit many of today's power devices which have complex functionality and miniaturized electronics packages leading to high heat fluxes. These high heat fluxes need to be managed, especially at localized high power regions that are referred to as hot-spots. Future power electronics are expected to have hot-spot regions on the die, which can generate heat fluxes exceeding 1000 $\mathrm{W} / \mathrm{cm}^{2}$ (i.e. more than six times the average heat flux on a current die. Without proper cooling techniques, such an increase in heat flux could lead to rise in hot-spot temperatures by $30^{\circ} \mathrm{C}$ or more than the average die temperature. [35 - 37] A temperature increase of that magnitude could be expected to severely limit the performance of the chip, ultimately leading to reduced life of the device. As a result, hotspot cooling has become a primary focus of thermal designs, often more important than decreasing the average temperature of the die.

Thermoelectric coolers seem a promising solution for hot spot thermal management because of their compact size, passive nature, high reliability, and ease of control [35 - 37]. Two drawbacks associated with use of thermoelectric coolers are their low cooling flux and their poor coefficient of performance (COP). One solution to achieving high cooling flux and minimizing the effect of the low COP is to spread 
the heat from the small hot spot to a larger thermoelectric device, thereby focusing the cooling. Wang et. al. have reported increasing cooling flux for hot-spot cooling in high power electronics with such a novel TE Mini-contact. For the chip package shown in figure 3.1 above, a 3D numerical model was used to investigate the thermal design. A heat flux of $1250 \mathrm{~W} / \mathrm{cm}^{2}$ was applied to hotspot, which had dimensions of $400 \mu \mathrm{m} \times 400 \mu \mathrm{m}$. The die thickness used in the study was $750 \mu \mathrm{m}$. A background heat flux of $70 \mathrm{~W} / \mathrm{cm}^{2}$ was applied to the die additionally.

Wang et. al. [35 - 37], reported that as the contact size is reduced, it will lead to increased cooling flux at the contact, directly correlated with area ratio between base of TEC device and the Mini-contact. Another advantage with use of minicontact based cooling is that the TE cooler can act to remove the heat generated from hot-spot only without removal of heat from the background, reducing the effect of low COP.

\subsubsection{Integration Options}

In the current study, the thermoelectric design team proposed two methods by which Mini-contact based TE cooling can be implemented for the case of a power electronic device constructed in a $3 \mu \mathrm{m}$ to $5 \mu \mathrm{m}$ thick epitaxial layer of GaN grown on SiC. The two proposed integration options are (1) Integrated SiC Mini-contact based TE cooling in which the mini-contact is machined directly out of the $\mathrm{SiC}$ base and attached to the TE cooler and (2) Discrete Mini-contact based TE cooling in which the mini-contact is fabricated separately of a material other than $\mathrm{SiC}$ and then attached to the SiC die and the TE cooler. 
The materials proposed for the discrete mini-contact include copper, diamond and silver-diamond composite, mainly because of their high thermal conductivity, which will be helpful in removal of heat. For the integrated Mini-contact option, the TE cooler is proposed to be attached to the integrated mini-contact via solder. On the other hand, the discrete Mini-contact will be attached via solder to both the TE cooler on one side and the SiC chip on the other. The above integration methods are illustrated in figure 3.2 below.

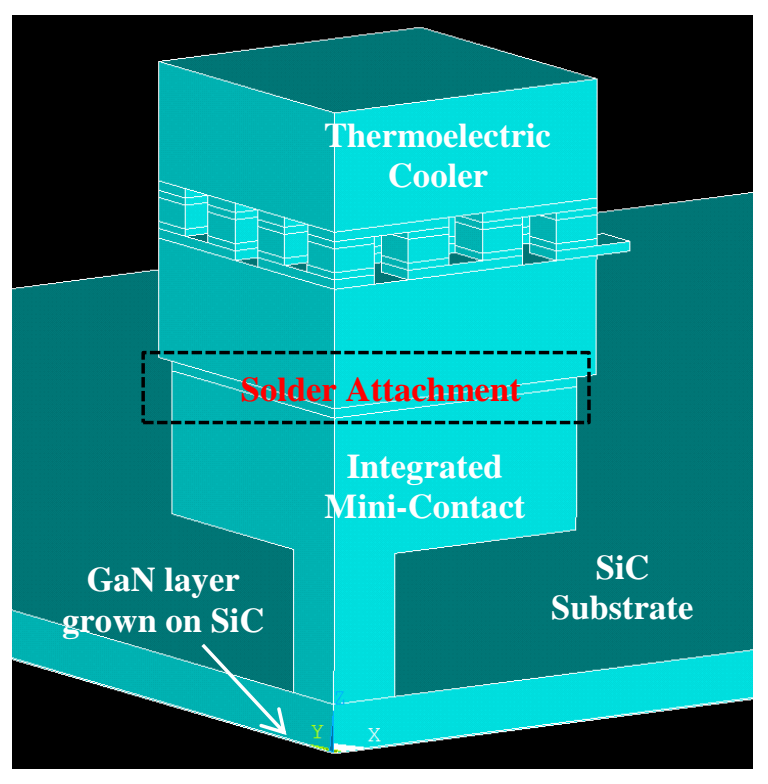

Figure 3.2: Integrated SiC Mini-contact Model (Quarter Symmetric)

This study focuses on a structural reliability assessment of the TE cooler/Solder Attach/Mini-contact/SiC/GaN die stack interconnections. SiC is known to be a very hard brittle material, which is highly elastic in nature. As an initial assessment, it was predicted that the failure due to thermo-mechanical stresses will be either at solder attachment level or inside the TE cooler. In later chapters, the detailed results of thermo-mechanical structural analysis will be presented. 


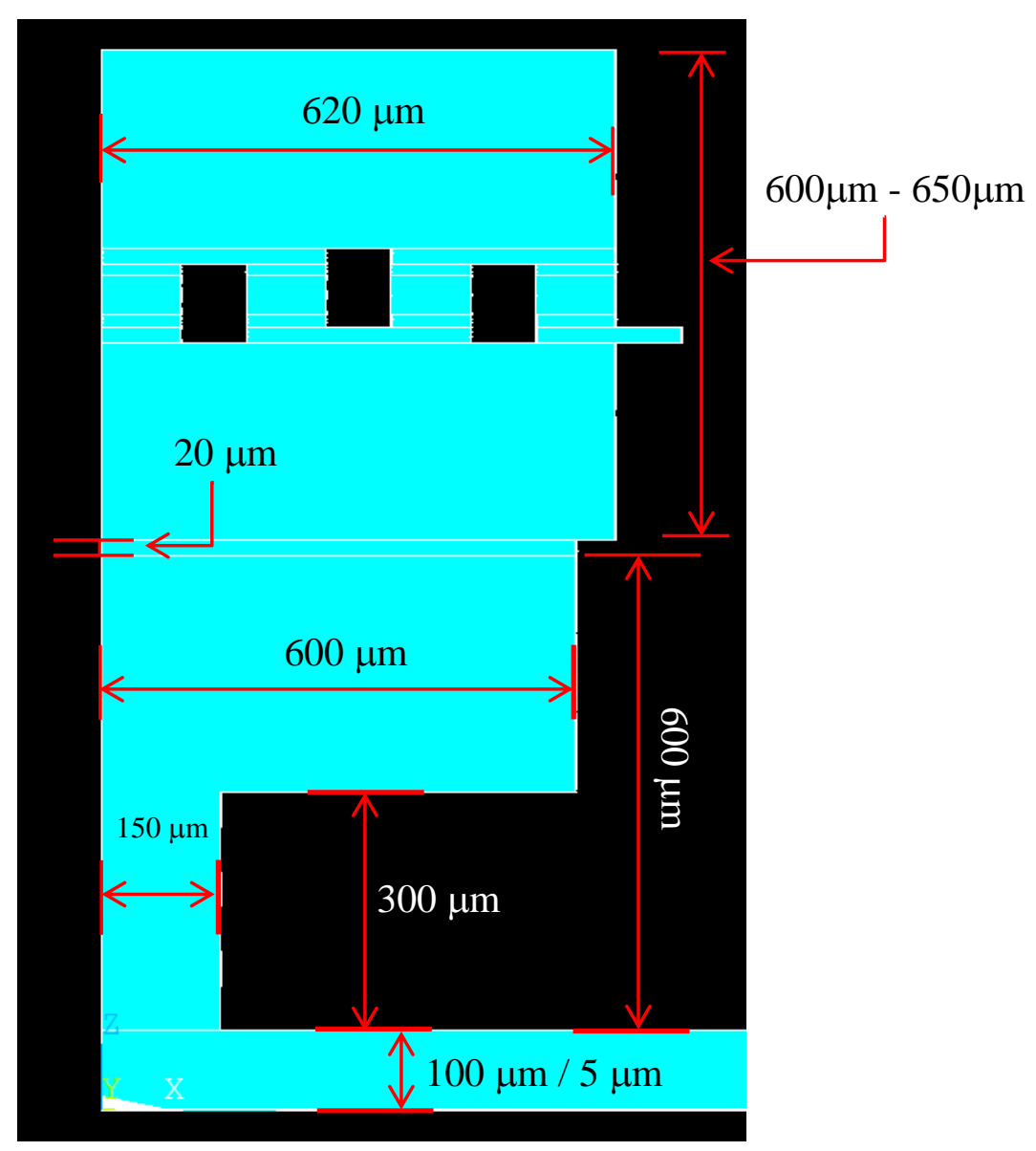

Figure 3.3: Approximate Package Dimensions (Side View, Half Symmetric)

As shown in figure 3.3 is the quarter symmetric view of the package FEA

model, cross-sectioned at the center of the hot spot $(200 \mu \mathrm{m}$ x $200 \mu \mathrm{m})$. In the current study, the dimensions of TEC device were supplied by Laird Technologies - for their HV 37 model. Detailed dimensional and material stack layer information for this device will be given in the later chapters. As mentioned previously, the above Tshaped Mini-contact design is based on earlier research [35 - 37]. A solder attachment of $20 \mu \mathrm{m}$ was used based on the rationale that the solder attach has $5 \mu \mathrm{m}$ Gold metallization on each side, with total thickness of $10 \mu \mathrm{m}$ along with $10 \mu \mathrm{m}$ of SAC 305 solder between the metallization. 
Three design geometries were studied for the discrete Mini-contact. These were the standard T-shape, along with a lofted shape and a tapered shape. They are presented schematically in the figure below. One can see that for integration of a discrete Mini-contact, we need solder attachment at both the top and the bottom. For both cases, a solder layer of thickness $20 \mu \mathrm{m}$ has been chosen based on the same rationale mentioned previously.

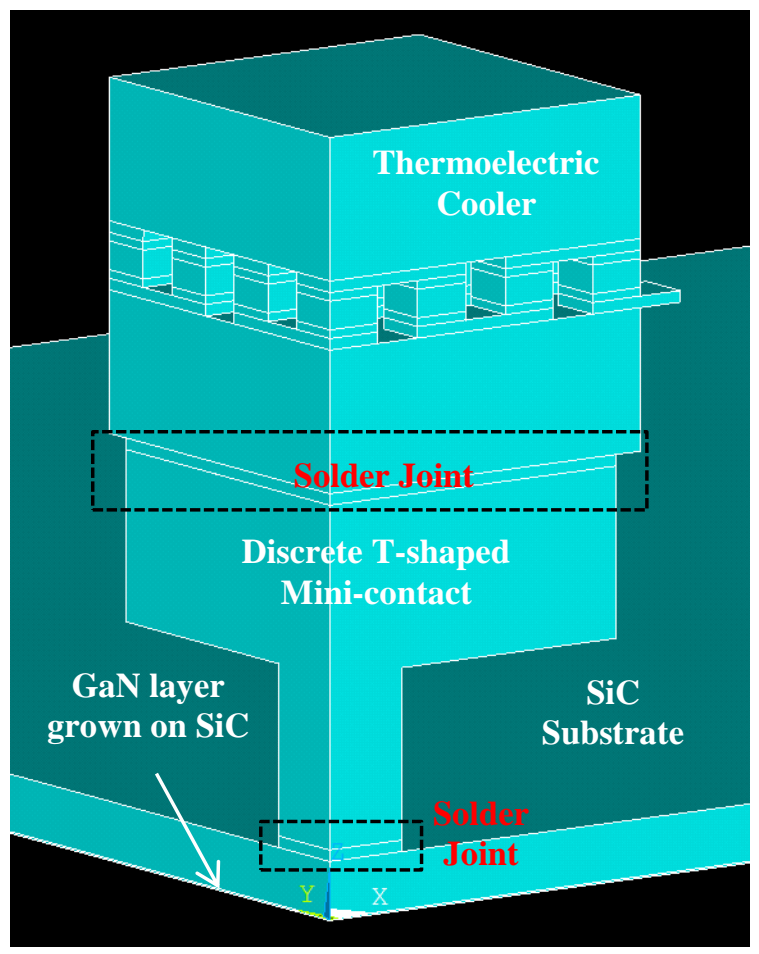

Figure 3.4: Discrete T-shaped Mini-contact Quarter Symmetric Model

A SAC 305 solder was chosen for the attachments because it is compatible with the materials and stable for temperatures up to $150^{\circ} \mathrm{C}$ to $200^{\circ} \mathrm{C}$. Also, for such a solder layer, elastic-plastic material properties are available, which is helpful in running a non-linear Finite Element based thermal-structural analysis. With such properties, one can evaluate whether the solder joint will undergo any plastic 
deformation, the extent of the plastic strain and its effect on the Mean Time to Failure of the solder joint.

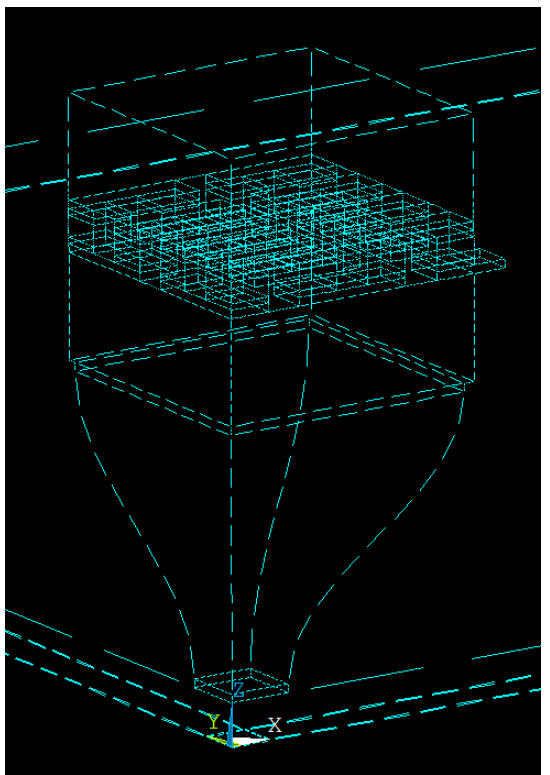

Figure 3.5: Lofted Mini-contact Quarter Symmetric Model

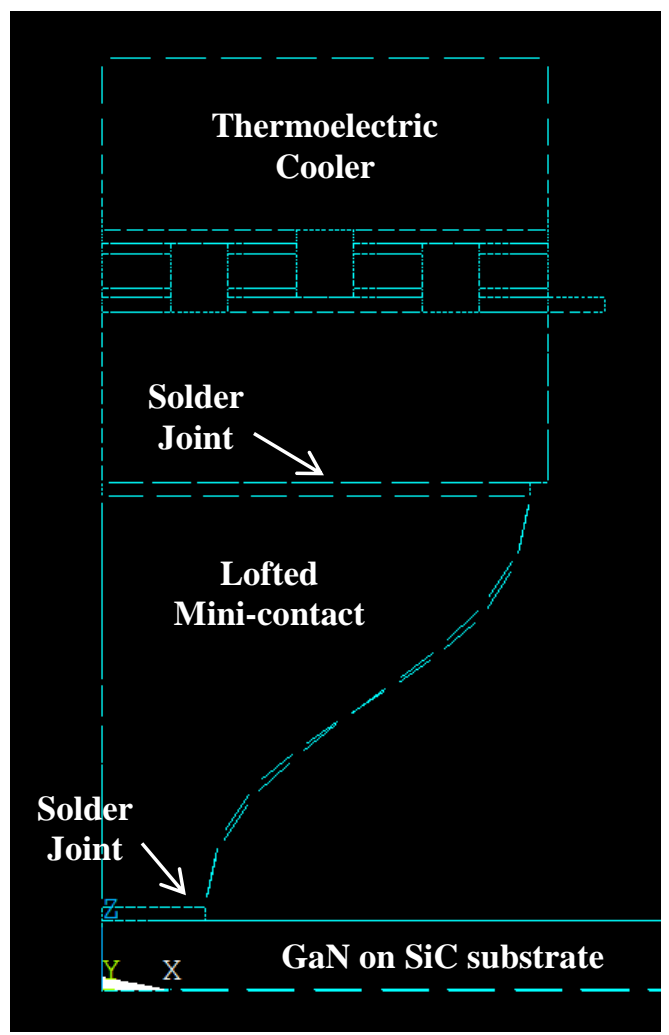

Figure 3.6: Side View - Lofted Mini-contact Model 
The lofted Mini-contact geometry is shown in figures 3.5 and 3.6 above and the tapered mini-contact geometry is shown in figures 3.7 and 3.8 below. These designs are based on optimization of thermal performance, which is related to how effectively heat can be removed from the hot-spot. The height of the lofted or the tapered Mini-contact is same as that of T-shaped Mini-contact, which is $600 \mu \mathrm{m}$. The width of the top and bottom bases of the lofted or the tapered Mini-contact is also the same as the T-shaped mini-contact, which are $1200 \mu \mathrm{m}$ and $300 \mu \mathrm{m}$ respectively. The Mini-contact covers a $200 \mu \mathrm{m}$ wide hot-spot area on GaN and $1200 \mu \mathrm{m}$ wide TEC area at top.

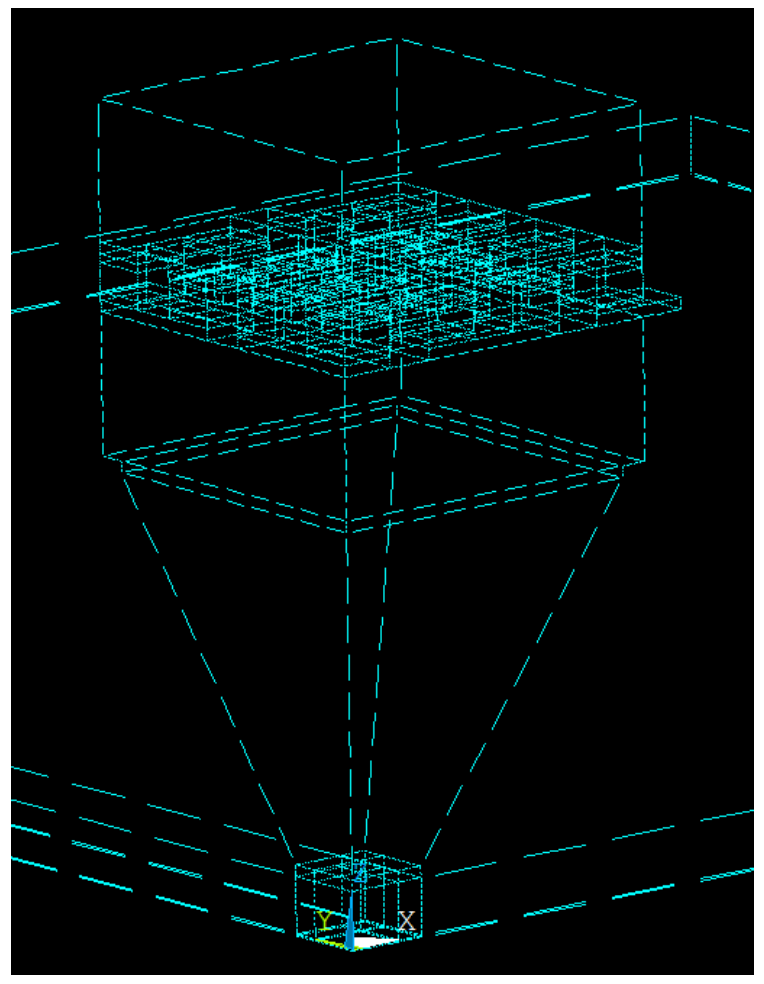

Figure 3.7: Taper Mini-contact Quarter Symmetric Model 


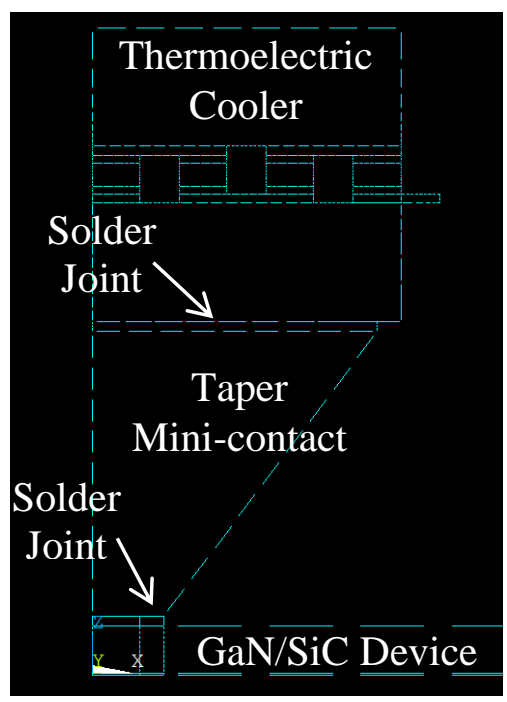

Figure 3.8: Side View - Taper Mini-contact Model

As mentioned earlier, the lofted Mini-contact model will help in heat removal efficiency and in turn reduce the hot-spot temperature. However, it's difficult to manufacture such complicated shapes. From a design for manufacturability standpoint, the taper Mini-contact geometry can fulfill this requirement.

\subsubsection{Material Selection}

From a product design perspective, material selection is important as it will help in reduction of cost and achieving the desired functionality. In the current research, there are two objectives behind selection of an appropriate material, which are good thermal management and structural reliability of the Mini-contact structure, which must last under the desired loading conditions for a million cycles. To achieve structural reliability, one needs to consider the importance of temperature gradients and difference in Coefficient of Thermal Expansion (C.T.E.) of various materials used in this Power Electronics package, as these factors will act as the driving force for thermo-mechanical stress generation. 
For thermoelectric Mini-contact based cooling of the hot-spot, a variety of materials have been proposed during the design phase. The primary candidates have been SiC, Copper, Diamond and Silver-Diamond composite. Copper has good thermal conductivity $(400 \mathrm{~W} / \mathrm{m}-\mathrm{K})$ and has found widespread use in applications, where a good thermal/electrical conductor is needed. From a structural standpoint, Copper is ductile in nature and will undergo plastic deformation to absorb stresses generated due to thermo-mechanical phenomena. With use of Copper as a Minicontact material, it is expected that failure will occur at the TE cooler level or solder attachment between TE cooler and SiC Mini-contact. However, one needs to account for the high C.T.E of Copper, which is about $16-18 \mathrm{ppm} / \mathrm{K}$ as compared to that of $\mathrm{SiC}$ die, about 3-4 ppm/K, and will result in a significant C.T.E. mismatch.

Comparatively, if the Mini-contact is assumed to be made up of $\mathrm{SiC}$, the failure site will shift in the Power Electronics package. $\mathrm{SiC}$ is a brittle material, with a very high Young's modulus of elasticity (480 GPa), which implies it's highly elastic in nature. With use of $\mathrm{SiC}$ as a Mini-contact material, one can suppose that the deformation site will transfer to the TE cooler level. However, it's essential to also consider the fact that $\mathrm{SiC}$ has a temperature dependent thermal conductivity. To be more specific, the thermal conductivity value reduces from an initial value of 430 $\mathrm{W} / \mathrm{m}-\mathrm{K}$ at room temperature to $\approx 260 \mathrm{~W} / \mathrm{m}-\mathrm{K}$ at a temperature of $100^{\circ} \mathrm{C}$. Also, the C.T.E. mismatch effect at chip level can be eliminated with use of an integrated $\mathrm{SiC}$ Mini-contact pillar, as the pillar will be grown on the $\mathrm{SiC}$ base.

Finally, diamond is known for its high thermal conductivity $(\approx 2000 \mathrm{~W} / \mathrm{m}-\mathrm{K})$ and high modulus of elasticity. Also, it's a brittle but strong material, so it may be a 
good initial assumption that the failure site will shift to the TE cooler level. Diamond also appears to be a suitable material from a structural reliability standpoint, as the C.T.E. of diamond (1-2 ppm/K) is comparable to that of $\mathrm{SiC}$, hence, the C.T.E. mismatch effect will not pose a major issue.

One of the important issues to be considered in this study is thermomechanical stress generation due to difference in C.T.E. across the material stack layers ( $\mathrm{SiC}$ die, Mini-contact, solder attachments and TE cooler stack). The major chunk of volume of TE cooler is consumed by the metallized ceramic conductors, which have a C.T.E. value of about $4.5 \mathrm{ppm} / \mathrm{K}$. Bismuth Telluride $\left(\mathrm{Bi}_{2} \mathrm{Te}_{3}\right) \mathrm{TE}$ elements are 40 - $50 \mu \mathrm{m}$ in thickness and have a C.T.E of about $18 \mathrm{ppm} / \mathrm{K}$. Thus, there is a local C.T.E. mismatch effect inside the cooler itself, and on the global scale there will be difference in C.T.E. between the TE cooler, the mini-contact, and the SiC substrate. Hence, the C.T.E. of the Mini-contact plays a major role in this study. One of the materials, which seems promising as a future application is SilverDiamond composite, where one can tailor the C.T.E of the material depending on the volume fraction of diamond particles present in the silver matrix.

\subsubsection{Silver-Diamond Composite as Thermal Management Material}

As mentioned previously, one can alter the C.T.E. of the silver-diamond composite material based on the volume fraction of diamond particles present in the silver matrix phase. The requirement of high thermal conductivity and a variable C.T.E. can be met with use of such a novel material. However, manufacturing of such composite material is still in research and development stage. There are various models available in literature for prediction of C.T.E. of particle-matrix composites. 
As an initial step, these models can be used for prediction of particle-matrix composite C.T.E. and they are listed as below.

\subsubsection{Rule of Mixtures for C.T.E. Prediction}

The rule of mixtures is a theoretical method, which can be used for prediction of various mechanical properties of a composite material (particle-reinforced composites, etc.) It gives an upper- and lower-bound on properties such as elastic modulus, mass density, ultimate tensile strength, thermal conductivity and electrical conductivity. For example, we can predict the elastic modulus using rule of mixtures as follows. [8]

The upper bound to modulus is given as follows.

$$
E_{c}(u)=E_{m} V_{m}+E_{p} V_{p}
$$

The lower bound or limit can be given as follows.

$$
E_{c}(l)=\frac{E_{m} E_{p}}{V_{m} E_{p}+V_{p} E_{m}}
$$

In the above equations, the letter $\mathrm{m}$ stands for matrix phase, which is Nanosintered Silver and the letter $\mathrm{p}$ stands for particle phase, which is Diamond. In order to make a first approximation to the theoretical properties of Diamond (60\%) - Silver (40\%) composite (particle phase: diamond, matrix phase: silver), the following properties (table 3.1), as available from literature [39, 40], were used. 
Table 3.1: Mechanical Properties of Diamond and Nano-Sintered Silver

\begin{tabular}{|c|c|c|c|c|c|c|}
\hline Material & $\begin{array}{c}\text { Thermal } \\
\text { Expansion } \\
\text { Coefficient } \\
\boldsymbol{\alpha} \\
\left(\mathbf{p p m}^{\left.* \mathbf{K}^{-1}\right)}\right.\end{array}$ & $\begin{array}{c}\text { Thermal } \\
\text { Conductivity } \\
\mathbf{k} \\
\left(\mathbf{W m}^{-\mathbf{1}} \mathbf{K}^{-\mathbf{1}}\right)\end{array}$ & $\begin{array}{c}\text { Elastic } \\
\text { Modulus } \\
\mathbf{E} \\
(\mathbf{G P a})\end{array}$ & $\begin{array}{c}\text { Poisson's } \\
\mathbf{R a t i o} \\
\mathbf{( v )}\end{array}$ & $\begin{array}{c}\text { Yield } \\
\text { Strength } \\
\mathbf{\sigma}_{\mathbf{y}} \\
(\mathbf{G P a})\end{array}$ & $\begin{array}{c}\text { Density } \\
\mathbf{\rho} \\
\mathbf{( 1 0}^{\mathbf{3}} \\
\left.\mathbf{k g} / \mathbf{m}^{\mathbf{3}}\right)\end{array}$ \\
\hline Diamond & 1 & 1800 & 1050 & 0.1 & 60 & 3.52 \\
\hline $\begin{array}{c}\text { Nano- } \\
\text { Sintered } \\
\text { Silver }\end{array}$ & 19.6 & 238 & 9 & 0.37 & 27 & 8.58 \\
\hline
\end{tabular}

Based on the rule of mixtures stated above, the upper and lower limits to elastic modulus of Diamond (60\%) - Silver (40\%) composite can be given as follows. The upper bound to modulus is given as follows.

$$
\begin{gathered}
E_{c}(u)=E_{m} V_{m}+E_{p} V_{p} \\
E_{c}(u)=\left(9 * 10^{9} * 0.6\right)+\left(1050 * 10^{9} * 0.4\right)=633.6 \mathrm{GPa} \cong 634 \mathrm{GPa}
\end{gathered}
$$

The lower bound or limit can be given as follows.

$$
\begin{gathered}
E_{c}(l)=\frac{E_{m} E_{p}}{V_{m} E_{p}+V_{p} E_{m}} \\
E_{c}(l)=\frac{1050 * 10^{9} * 9 * 10^{9}}{\left[\left(0.4 * 1050 * 10^{9}\right)+\left(0.6 * 9 * 10^{9}\right)\right]}=22.2 \mathrm{GPa} \cong 22 \mathrm{GPa}
\end{gathered}
$$

As one can infer from the moduli values above, there is a pretty wide gap in the lower and upper bound elastic moduli values. Similarly, we can predict the Poison's ratio to be 0.21 (upper limit) as a first approximation.

The thermal conductivity and coefficient of thermal expansion for a composite material with such a volume fraction is based on the works of Abyzov [43]. They studied the thermo-mechanical properties of $\mathrm{W}$ and Mo coated diamond in metal matrices. It was suggested that these two elements will facilitate composites with high 
thermal conductivities due to their inherent thermal conductivity as compared to other carbide-forming elements. Thermal conductivity for Diamond (60\%) - Silver (40\%) composite was predicted as $834 \mathrm{~W} / \mathrm{m}-\mathrm{K}$ and with a coefficient of thermal expansion of $8.6 \mathrm{ppm} / \mathrm{K}$.

\subsubsection{Other Models for C.T.E. Prediction}

There are various models available in literature for prediction of thermal expansion coefficient of particle matrix composites. [44, 45] Some of the widely accepted models include Schapery Model for spherical particles, Turner Solution for two-dimensional models and Levin's Model for any isotropic two phase composites. These models are based on the assumption that there is perfect adhesion between the particle and matrix phase.

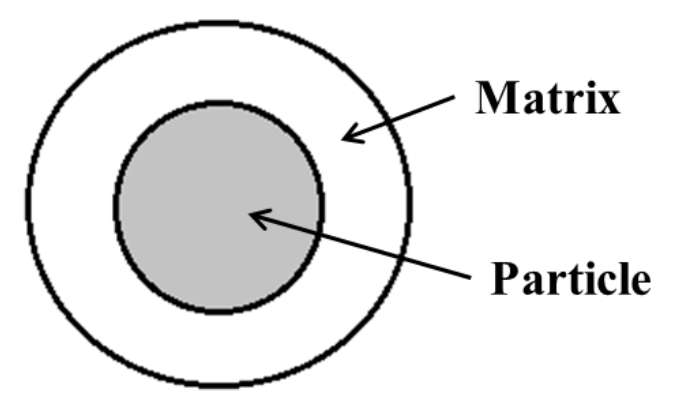

Figure 3.9: Unit Model Representation of Particle - Matrix Phases

Schapery Model is given as below. It assumes that the particles are wetted by uniform, isotropic, homogeneous matrix.

$$
\beta_{c}=\beta_{m} V_{m}+\beta_{p} V_{p}-\left(\beta_{m}-\beta_{p}\right) V_{p} A
$$

where,

$$
A=\left(\frac{1}{K_{m}}-\frac{1}{K_{p}}\right)\left(\frac{V_{p}}{K_{m}}+\frac{V_{m}}{K_{p}}+\frac{3 G_{m}}{4}\right)^{-1}
$$


The subscripts represent different parameters in the above equation. $\mathrm{K}$ is bulk modulus, $\mathrm{G}$ is shear modulus, $\mathrm{c}$ is composite, $\mathrm{m}$ is matrix, $\mathrm{p}$ is particle reinforcement and $\mathrm{v}$ is volume fraction in \%. [63] Turner Solution gives the following equation for prediction of C.T.E. of particle reinforced composite.

$$
\alpha_{c}=\frac{\alpha_{m} V_{m} K_{m}+\alpha_{p} V_{p} K_{p}}{V_{m} K_{m}+V_{p} K_{p}}
$$

where,

$\mathrm{K}$ is bulk modulus, $\mathrm{c}$ is composite, $\mathrm{m}$ is matrix, and $\mathrm{p}$ stands for particle reinforcement.

\subsubsection{Prediction of Thermal Conductivity using FE Analysis}

A simple 3-D Finite Element analysis was conducted to predict the thermal conductivity of Silver-Diamond composite. For this analysis, it was assumed that the particle shape is spherical and the particles are uniformly distributed in the Silver matrix phase. The attachment between particle and matrix was assumed perfect with no interfacial layer present. Furthermore, it was assumed that the diamond particles and silver matrix are homogeneous isotropic materials.

In the FE model, the number of particles in the $\mathrm{X}, \mathrm{Y}$ and $\mathrm{Z}$ directions were considered to be three with spacing between individual particles to be $6 \mu \mathrm{m}$ and 8 $\mu \mathrm{m}$. The diamond particles were assumed to be $4 \mu \mathrm{m}, 5 \mu \mathrm{m}, 6 \mu \mathrm{m}$ and $7 \mu \mathrm{m}$ in diameter. Hence the respective diamond volumes fractions considered were $18 \%$, $28 \%, 44 \%$ and $54 \%$. A pictorial view of uniform diamond particle phase and silver matrix is represented as shown below in figure 3.10. 

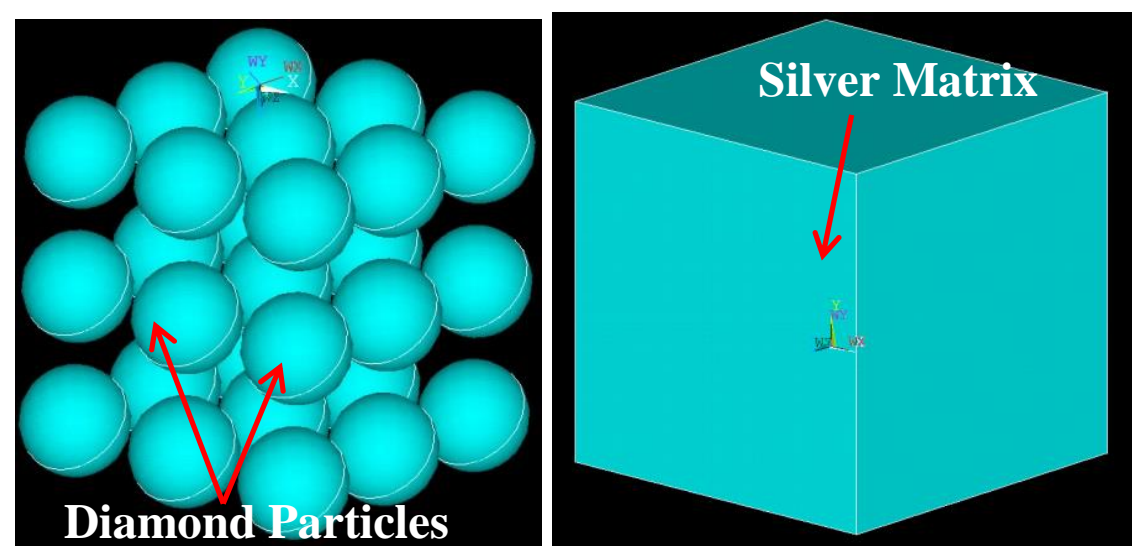

Figure 3.10: FE Model Setup for Prediction of Mechanical Properties

A thermal and a corresponding thermo-mechanical stress analysis were

performed to give an approximate prediction of the thermal conductivity and stress at particle-matrix interface.

Outside Wall

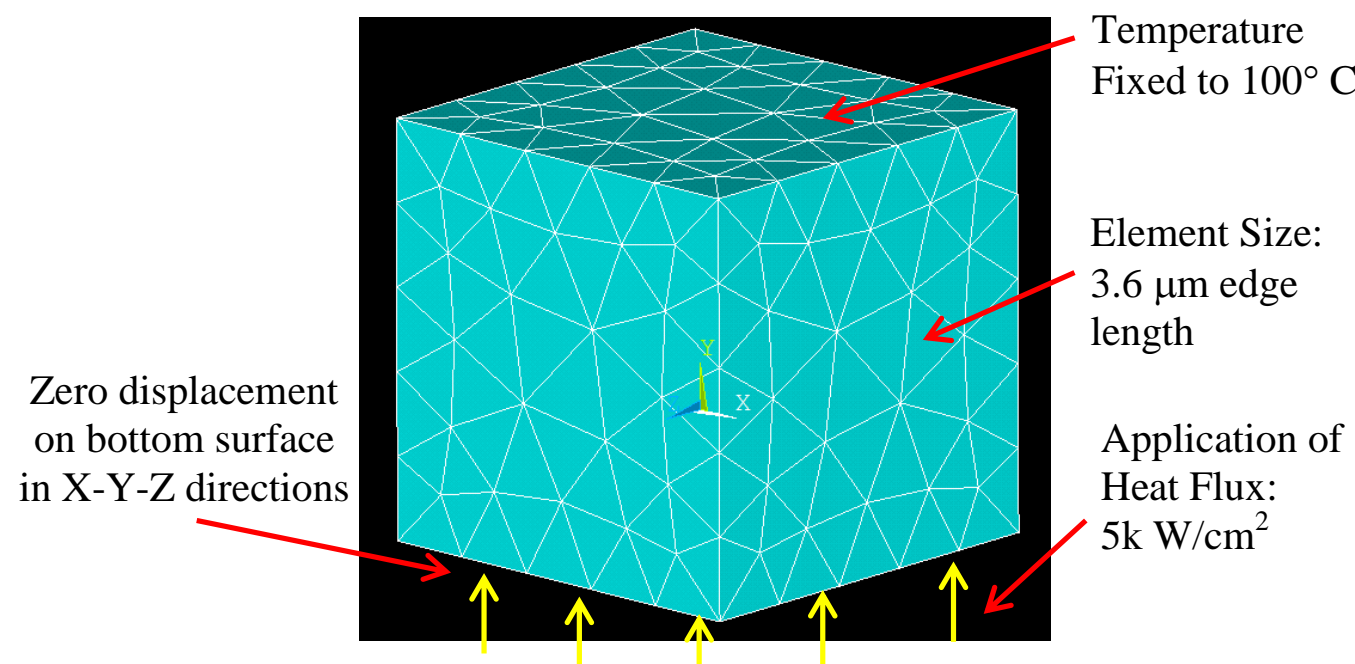

Figure 3.11: Illustration of Application of Boundary Conditions (Thermal/Structural)

The effective thermal conductivity was predicted based on Newton's law of cooling. The temperature differential is shown as below. 


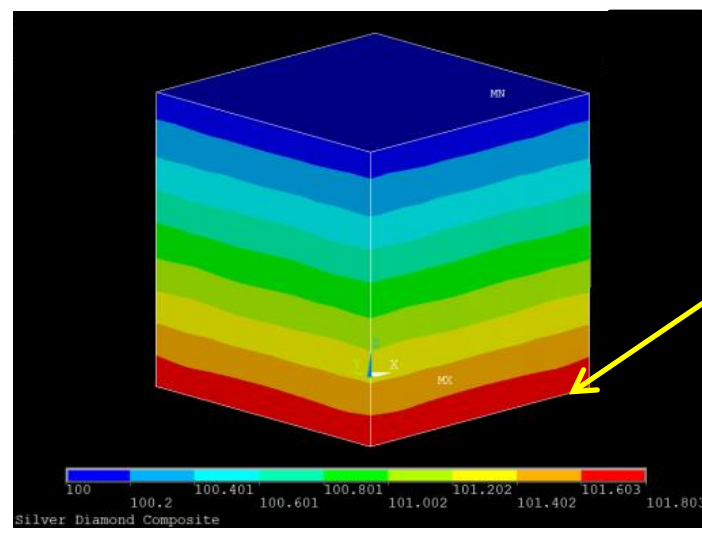

Temperature

Difference

$\Delta \mathrm{T}=1.8^{\circ} \mathrm{C}$

Figure 3.12: Temperature Differential for Particle Size $(\Phi)$ of $4 \mu \mathrm{m}$

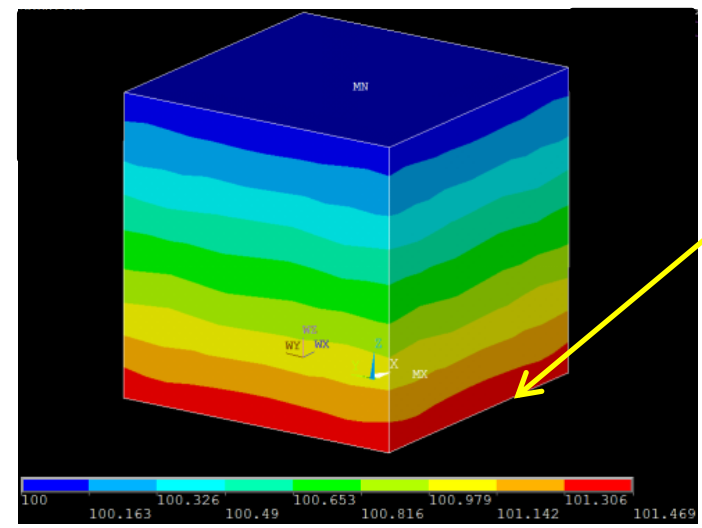

Temperature

Difference

$\Delta \mathrm{T}=1.47^{\circ} \mathrm{C}$

Figure 3.13: Temperature Differential for Particle Size $(\Phi)$ of $5 \mu \mathrm{m}$

The effective thermal conductivity can be predicted based on Newton's law of cooling as follows.

$$
K_{\text {effective }}=\frac{q^{\prime \prime} \times \Delta T}{d t}
$$

where,

q" is applied heat flux, $\Delta \mathrm{T}$ is difference in temperature across a thickness of dt. 
Table 3.2: Diamond Particle Spacing and Size Comparison

\begin{tabular}{|c|c|c|c|c|}
\hline \multirow{2}{*}{ Parameter } & \multicolumn{2}{|c|}{ Inter-particle Spacing = $6 \mu \mathrm{m}$} & \multicolumn{2}{|c|}{ Inter-particle Spacing $=8 \mu \mathrm{m}$} \\
\hline & $\Phi=4 \mu \mathrm{m}$ & $\Phi=5 \mu \mathrm{m}$ & $\Phi=6 \mu \mathrm{m}$ & $\Phi=7 \mu \mathrm{m}$ \\
\hline $\begin{array}{l}\text { Diamond } \\
\text { Volume } \\
\text { Fraction }\end{array}$ & $18 \%$ & $44 \%$ & $28 \%$ & $54 \%$ \\
\hline $\begin{array}{c}\text { Effective } \\
\text { Thermal } \\
\text { Conductivity }\end{array}$ & $499 \mathrm{~W} / \mathrm{m}-\mathrm{K}$ & $612 \mathrm{~W} / \mathrm{m}-\mathrm{K}$ & $548 \mathrm{~W} / \mathrm{m}-\mathrm{K}$ & $655 \mathrm{~W} / \mathrm{m}-\mathrm{K}$ \\
\hline $\begin{array}{c}\text { Von-Mises } \\
\text { Stress }\end{array}$ & $\begin{array}{c}11.6-12.7 \\
\mathrm{MPa}\end{array}$ & $\begin{array}{c}12.4-13.5 \\
\mathrm{MPa}\end{array}$ & $\begin{array}{c}13.8-15.2 \\
\mathrm{MPa}\end{array}$ & $\begin{array}{c}14.8-16.3 \\
\mathrm{MPa}\end{array}$ \\
\hline $\begin{array}{c}\text { Max. } \\
\text { Principal } \\
\text { Stress } \\
\text { (Compressive) }\end{array}$ & $\begin{array}{c}31.5-39.6 \\
\mathrm{MPa}\end{array}$ & $\begin{array}{c}36.8-46.4 \\
\mathrm{MPa}\end{array}$ & $22-28 \mathrm{MPa}$ & $\begin{array}{c}28.6-35.9 \\
\mathrm{MPa}\end{array}$ \\
\hline $\begin{array}{c}\text { Von-Mises } \\
\text { Plastic Strain }\end{array}$ & $\begin{array}{c}5.82 \mathrm{e}-3- \\
6.49 \mathrm{e}-3 \\
\end{array}$ & $\begin{array}{l}5.95 \mathrm{e}-3- \\
6.62 \mathrm{e}-3 \\
\end{array}$ & $\begin{array}{c}1.09 \mathrm{e}-2- \\
1.22 \mathrm{e}-2\end{array}$ & $\begin{array}{l}6.27 \mathrm{e}-3- \\
7.66 \mathrm{e}-3\end{array}$ \\
\hline $\begin{array}{c}\text { Max. } \\
\text { Principal } \\
\text { Strain }\end{array}$ & $1.78 \mathrm{e}-4$ & $2.83 \mathrm{e}-4$ & $2.77 \mathrm{e}-4$ & $3.28 \mathrm{e}-4$ \\
\hline
\end{tabular}

The following figure illustrates the stress/strain contour plot across the particle-matrix interface.

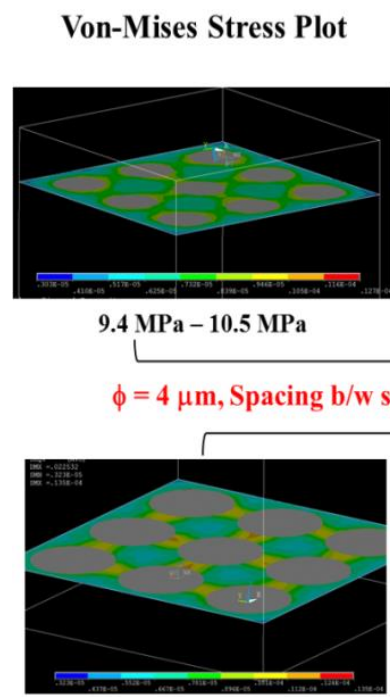

12.4 MPa - 13.5 MPa
Plastic Strain Plot

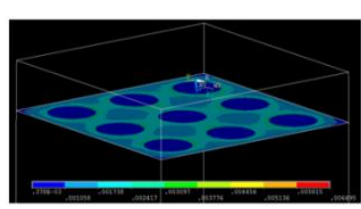

$3.09 \times 10^{-3}-3.76 \times 10^{-3}$
Max. Principal Stress Plot ( $3^{\text {rd }}$ Principal Stress)

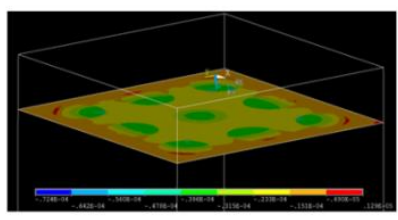

$-39 \mathrm{MPa}$ to $-35 \mathrm{MPa}$ $\phi=5 \mu \mathrm{m}$, Spacing $\mathrm{b} / \mathrm{w}$ spheres $=6 \mu \mathrm{m}$

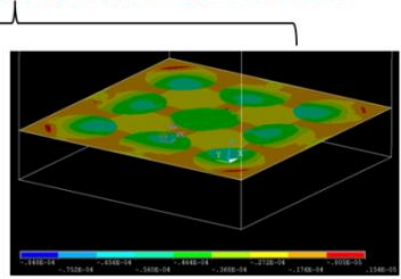

$-46 \mathrm{MPa}$ to $-37 \mathrm{MPa}$

Figure 3.14: Illustration of Stress/Strain Plot at Diamond Particle - Silver Matrix Interface for various Size and Spacing Options in Thermo-mechanical Analysis 


\subsubsection{Functionally Graded Silver-Diamond Composite}

Since the main reliability issue from a structural reliability standpoint is generation of thermo-mechanical stresses due to difference in C.T.E. at a global level between the TE Cooler, the mini-contact, and the $\mathrm{SiC} / \mathrm{GaN}$ device along with the presence of temperature gradient of about $25^{\circ} \mathrm{C}$ across the profile, a solder attachment should be chosen, if available, which will not fail under the given loading conditions. Based on thermo-mechanical fatigue failures observed in power electronics package, it is observed that the failure in device often occurs at the attachment level, initially by micro-crack (fatigue) formation. Hence, there is a need for compliant attachment which can absorb theses stresses, and not fail by fatigue crack formation. One of the solutions to the C.T.E. mismatch problem is to use a functionally graded SilverDiamond composite as both the Mini-contact and attach, as shown in figure 3.15 below.

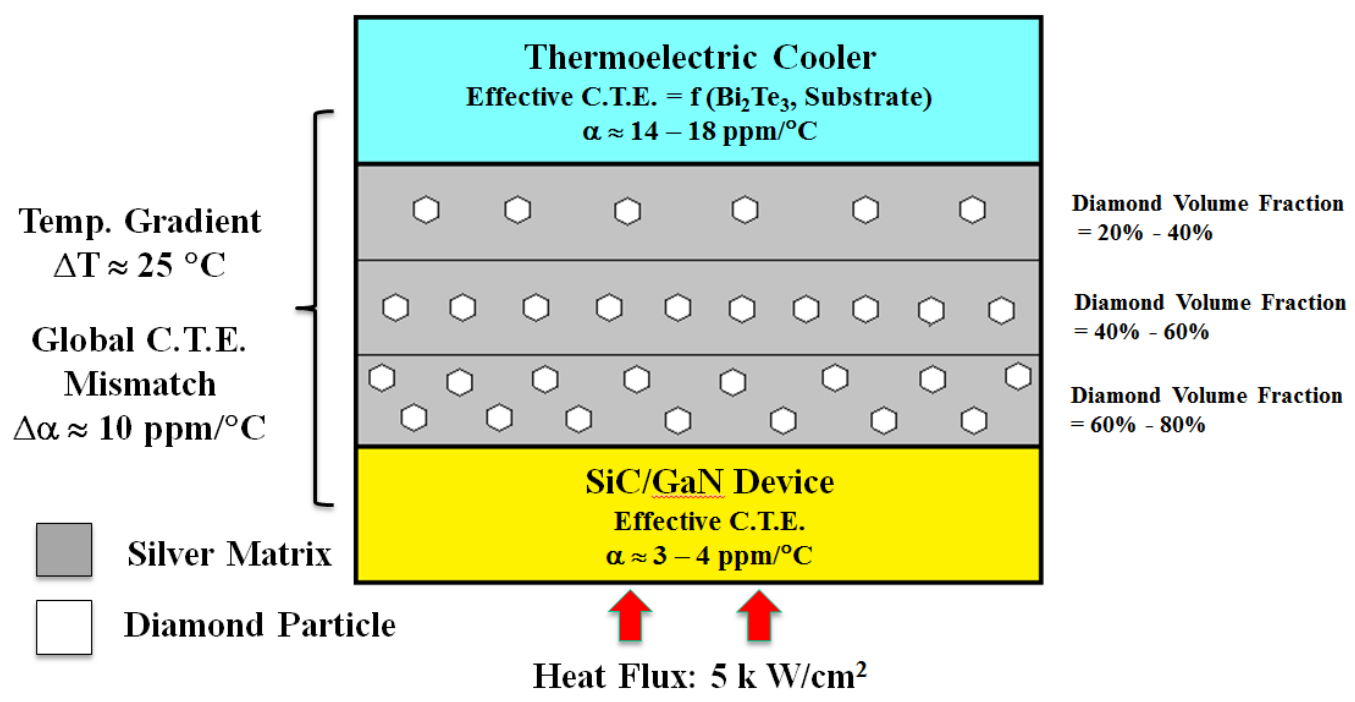

Figure 3.15: Functionally Graded Metal-Diamond Composite Concept 
As it can be inferred, with use of a functionally graded composite, we can tailor the properties of the composite material as per the desired requirement. For the above problem, we have a situation, where we need high diamond volume fraction at the $\mathrm{SiC}$ base level to provide a low CTE as compared to that of silver matrix. This will also help in removal of heat from the hot-spot region due to the high thermal conductivity of diamond, and based on rule of mixtures, one can approximate that the C.T.E. of the material in this region will be close to that of $\mathrm{SiC}$ die. At the level close to TE Cooler, we'll need a lower volume fraction of diamond to produce a material with a higher C.T.E. value, close to that of TE Cooler itself and in the range of 16 $18 \mathrm{ppm} / \mathrm{K}$. Also, with a higher volume fraction of silver matrix phase near to the TE cooler, we can ensure a good attachment to the TE Cooler. The above concept of functionally graded Silver-Diamond composite seems to be a good solution to the problem of C.T.E. mismatch, but one needs to understand that manufacturability of such a material is still in a research and development phase. Also, there will be issues of thermal boundary resistance across diamond particle and silver matrix interfaces, which will need to be addressed by use of a proper adhesive material layer between the diamond particle and silver matrix phase. 


\section{Chapter 4: Finite Element Model and Boundary Conditions}

In this chapter, the Finite Element (FE) model setup for thermo-mechanical structural reliability modeling will be presented. The modeling process is divided into several steps, namely, (1) CAD model setup, (2) Material properties input, (3) Selection of element type and mesh generation, (4) Application of thermal and mechanical boundary conditions and (5) Interpretation of modeling results. Details of steps (1) - (4) will be explained in this chapter.

\subsection{CAD Model Setup}

Finite Element (FE) analysis is a numerical method for obtaining approximate solutions to a given problem. The thermo-mechanical stress analysis problem is divided into two separate studies, thermo-electric based Mini-contact cooling and thin-film manifold - microchannel cooling.

For thermo-electric Mini-contact based cooling, the mechanism of interest is thermo-mechanical cyclic fatigue. The driving force to thermo-mechanical stress generation is the coefficient of thermal expansion mismatch of different materials used for packaging. The integration levels of interest from a structural reliability standpoint include (1) Mini-contact integration with SiC chip, (2) thermo-electric cooler integration with Mini-contact and lastly, (3) integration of thermo-electric elements with metallized Copper deposited on AlN headers. The integration at various levels is assumed to be done through a $20 \mu \mathrm{m}$ thick joint of SAC305 solder. SAC305 solder is a lead-free alloy that contains $96.5 \%$ Tin, $3 \%$ Silver and $0.5 \%$ Copper and is known to be a reliable option for high temperature $\left(100{ }^{\circ} \mathrm{C}-150{ }^{\circ} \mathrm{C}\right)$ 
applications [30]. For manifold-microchannel cooling, the failure mechanism of interest is brittle fracture due to stress concentrations at micro-fin corners. The stresses are generated due to the temperature gradient across the Silicon Carbide micro-fin region.

This thermo-mechanical problem was solved using commercial FEA software, ANSYS Mechanical APDL, release 15.0, Academic Research version. Use of both command language and GUI was made to perform the analysis. [55]

\subsubsection{Thermo-electric Mini-contact based Cooling}

For ease of modeling and computational time purposes, a quarter symmetric CAD model was used to represent the whole package geometry. With the help of such a model, one can reduce the number of equations generated while solving the FE code as compared to the number of equations generated with a full model. Figure 4.1 gives an overview of the quarter symmetric CAD model setup. Different regions of the CAD model assembly are explained with the help of following figures.

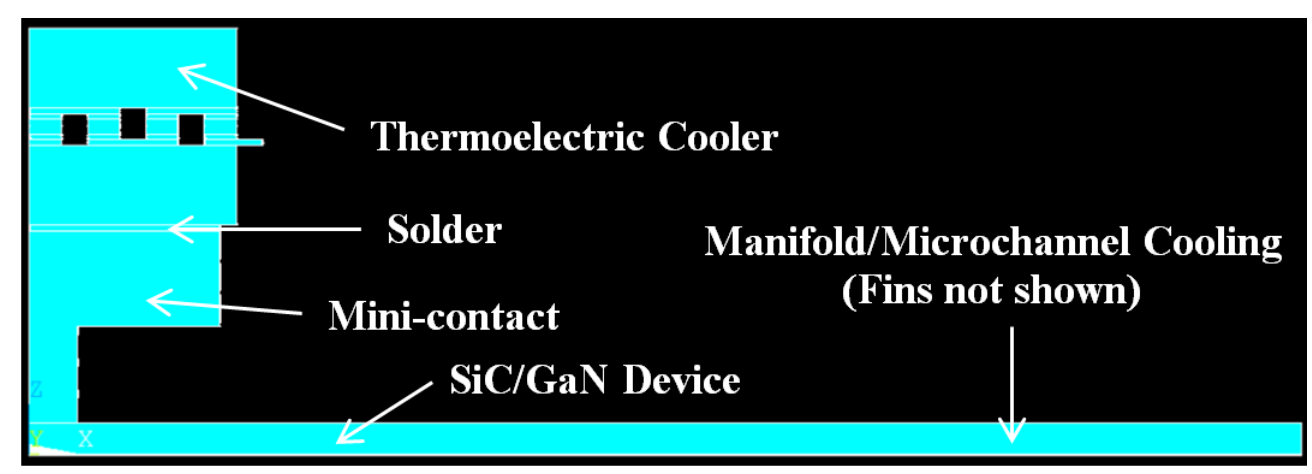

Figure 4.1: CAD Model Package

The complete package is supposed to be connected to the printed circuit board on the front side (or underside) of the $\mathrm{GaN}$ on $\mathrm{SiC}$ substrate using a flip chip solder joint interconnection. As shown below in figure 4.2, the model consists of a $3 \mu \mathrm{m}$ 
layer of $\mathrm{GaN}$ on $\mathrm{SiC}$ substrate. During manufacturing of such a power electronics device, $\mathrm{GaN}$ is deposited as an epitaxial layer on the $\mathrm{SiC}$ substrate.

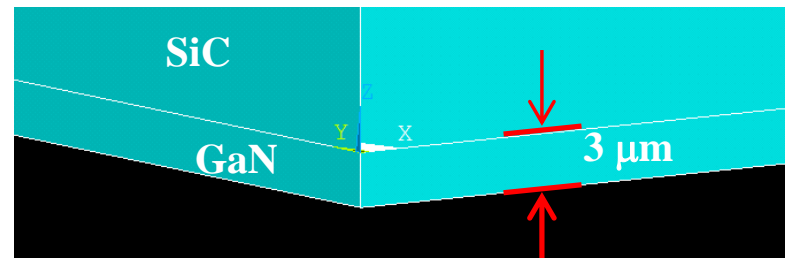

Figure 4.2: $\mathrm{GaN}$ on $\mathrm{SiC}$

The dimensions of the $\mathrm{SiC}$ die are shown below in figure 4.3. As it can be inferred from the diagram below, the die has a footprint of $10 \mathrm{~mm}$ x $10 \mathrm{~mm}$ with a thickness of $100 \mu \mathrm{m}$. With a quarter symmetric CAD model option, the dimensions are $5 \mathrm{~mm}$ x $5 \mathrm{~mm}$. The dimensions of three different Mini-contact options are shown below in figure 4.4 .

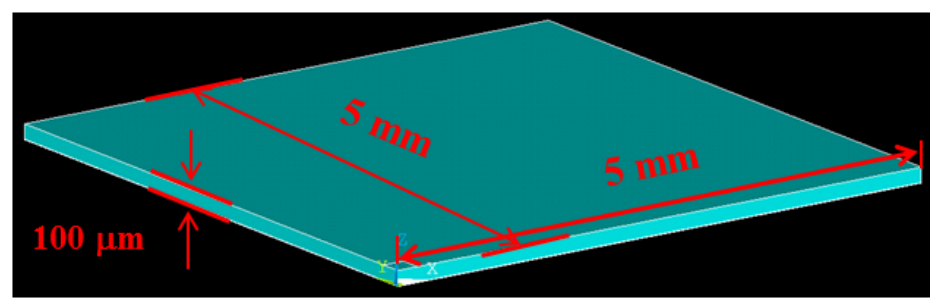

Figure 4.3: SiC die Quarter Symmetric Dimensions

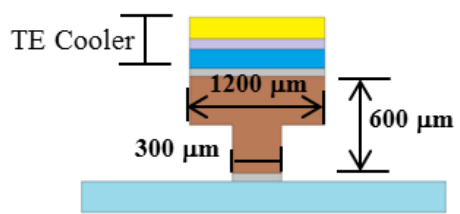

T-shaped Geometry

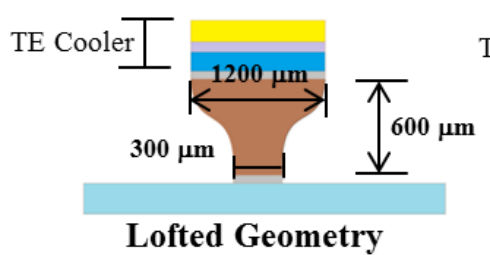

Lofted Geometry

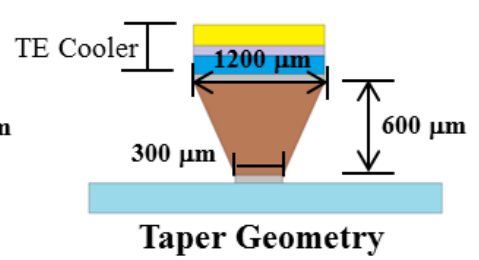

Taper Geometry

Figure 4.4: T-shaped, Lofted and Taper Mini-contact Dimensions

The Mini-contact dimensions are based on the research reported by Yang, Bar-Cohen and Wang [35]. The lofted and taper Mini-contact geometries are based on the idea of enhancement in heat spreading from the hot-spot region to the base of 
TE Cooler. The heights of top and bottom bases of the T-shaped Mini-contact are 300 $\mu \mathrm{m}$ each. Base widths are $1200 \mu \mathrm{m}$ (top base) and $300 \mu \mathrm{m}$ (bottom base) for all options.

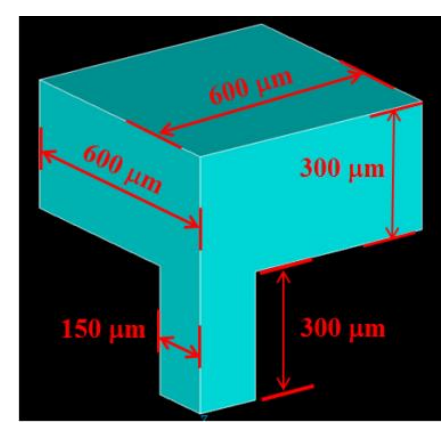

Figure 4.5: T-shaped Mini-contact, Quarter Symmetric CAD model

The discrete Mini-contact is integrated with SiC chip via a $20 \mu \mathrm{m}$ thick joint of solder attach. The $20 \mu \mathrm{m}$ dimension is a commonly used bond line for power electronics packaging. Overall, it gives low thermal resistance and is the smallest bond line possible from a reliability standpoint. With a $20 \mu \mathrm{m}$ solder joint, the intermetallic layer is assumed to be around $5 \mu \mathrm{m}-10 \mu \mathrm{m}$ in thickness along with a solder joint thickness of $10 \mu \mathrm{m}-15 \mu \mathrm{m}$. Figure 4.5 explains schematically dimensions of the solder joint used for TE Cooler integration with Mini-contact.

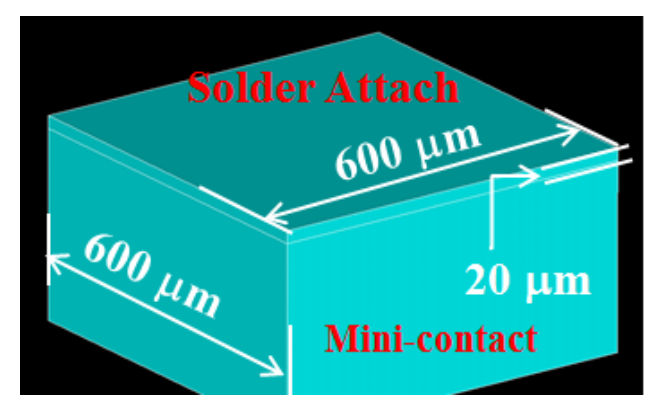

Figure 4.6: Solder on Mini-contact for TE Cooler integration 
The thermoelectric cooler used for cooling hot-spot heat flux of $5 \mathrm{k} \mathrm{W} / \mathrm{cm}^{2}$ via the Mini-contact is shown in figure 4.6 below. The TE cooler was supplied from Laird technologies and it's their HV 37 thin film model [46].

\begin{tabular}{|c|c|}
\hline $250 \mu \mathrm{m}$ & AIN header \\
\hline $10-20 \mu \mathrm{m}$ & Copper \\
\hline $10-20 \mu \mathrm{m}$ & Solder Attach \\
\hline $40-60 \mu \mathrm{m}$ & $\begin{array}{l}\text { N/P type Elements } \\
\text { Bismuth Telluride }\end{array}$ \\
\hline $10-20 \mu \mathrm{m}$ & Solder Attach \\
\hline $10-20 \mu \mathrm{m}$ & Copper \\
\hline $250 \mu \mathrm{m}$ & AIN header \\
\hline
\end{tabular}

Figure 4.7: HV 37 Laird Thin Film Device

As one can infer from figure 4.6 above, the major bulk of thermo-electric cooler are the headers $(250 \mu \mathrm{m}$ each) made up from Aluminum Nitride (AlN). The Bismuth Telluride $\left(\mathrm{Bi}_{2} \mathrm{Te}_{3}\right)$ elements are at the heart of the thermo-electric device and they are interconnected to the AIN headers via depositing solder (assumed to be SAC 305) on metallized Copper on AlN headers. The SAC305 solder joint is assumed to be $20 \mu \mathrm{m}$ thick for same reasons given above for integration of Mini-contact. The CAD model of the TE cooler is shown below in figure 4.7. [46]

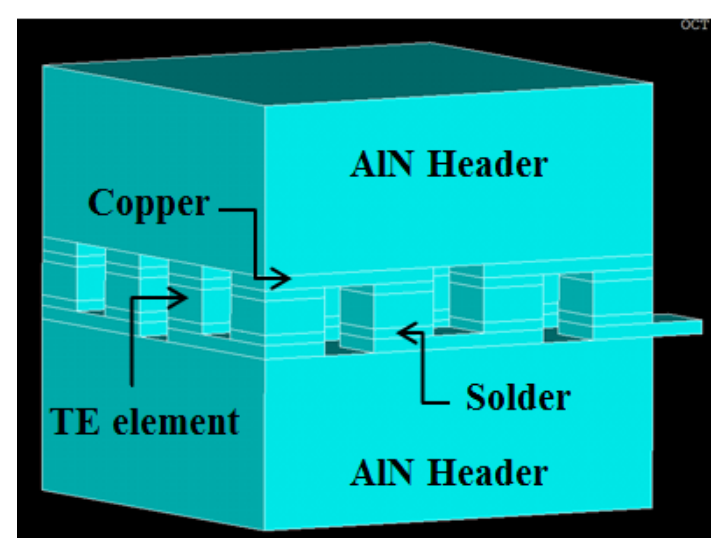

Figure 4.8: Thermo-electric Cooler CAD model (HV 37 model) 


\subsubsection{Manifold-Microchannel Cooling}

The manifold-microchannel cooling takes advantage of the force fed cooling technology reported by Ohadi, Cetegen et. al. [38]. An overview of the assembly is shown below in figure 4.8 .

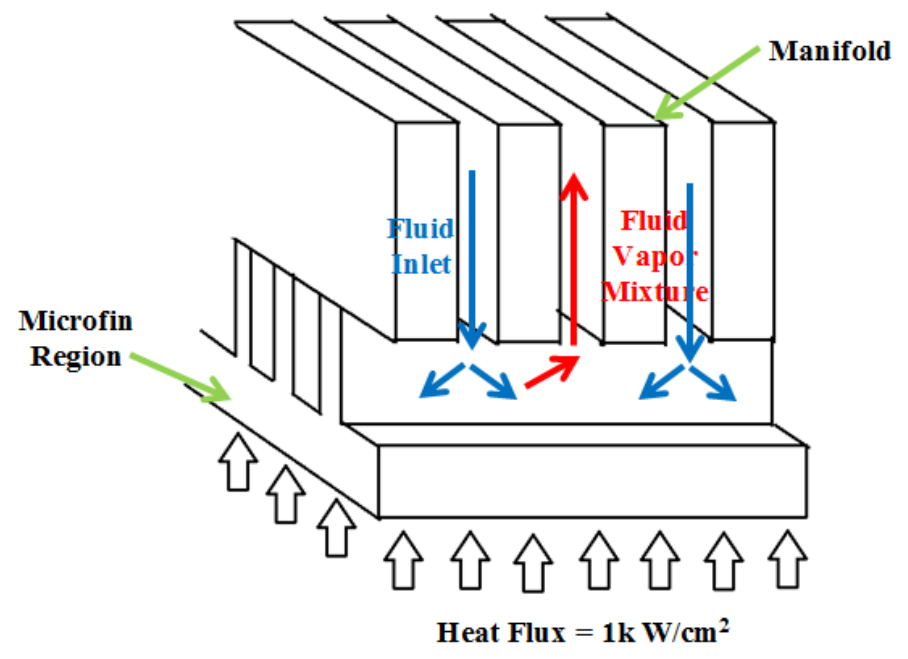

Figure 4.9: Manifold - Microchannel Model - Forced Fed Cooling Technology [38]

For FE modeling purposes, a unit cell model was used for performing the stress analysis. The dimensions of the unit cell model are shown below in figure 4.9.

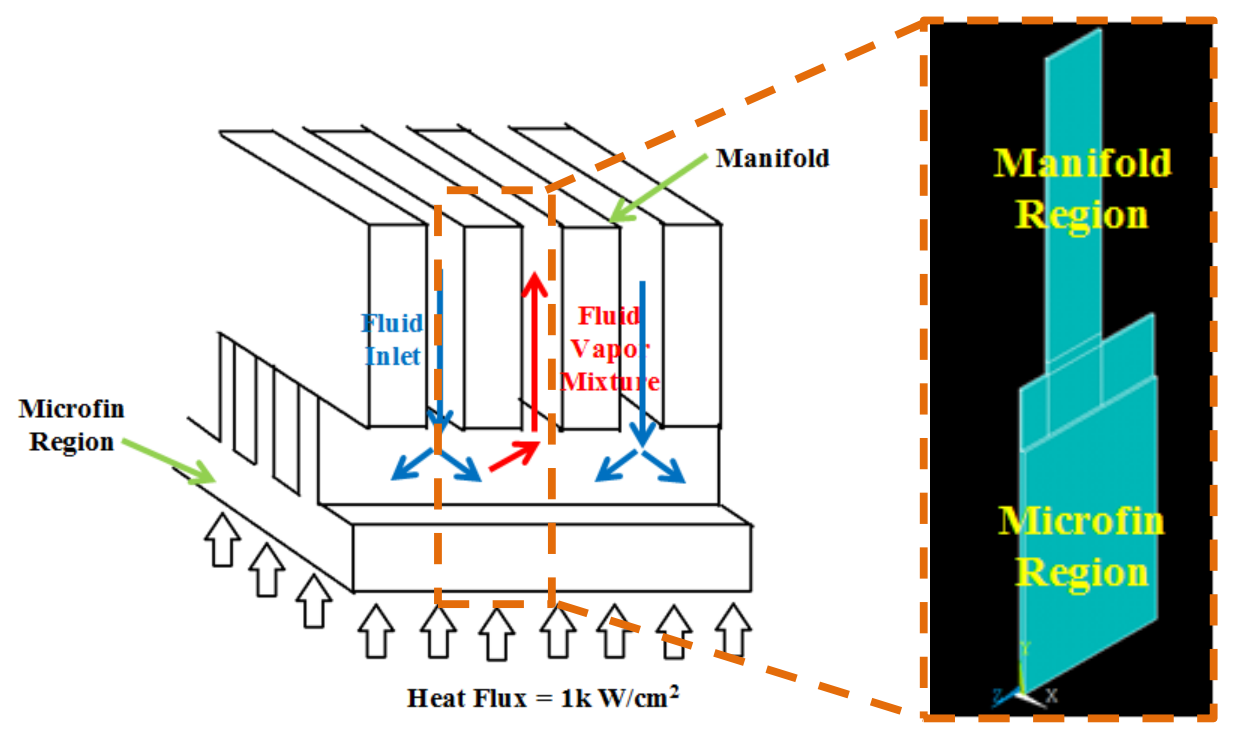

Figure 4.10: Unit Cell Model - Manifold Microchannel Cooling 
The unit cell model shown above is a representative of several cells of the complete microchannel. A half unit cell model was used for ease of modeling purposes. The dimensions of the micro-channel base and fin are shown below in figure 4.10. As one can infer from the diagram below, the fin width is in range of 0 $100 \mu \mathrm{m}(10 \mu \mathrm{m}$ used for this case) and the micro-channel base width was in range of 0 - $100 \mu \mathrm{m}$ (set as $20 \mu \mathrm{m}$ ). In unit cell model, half dimensions were used.

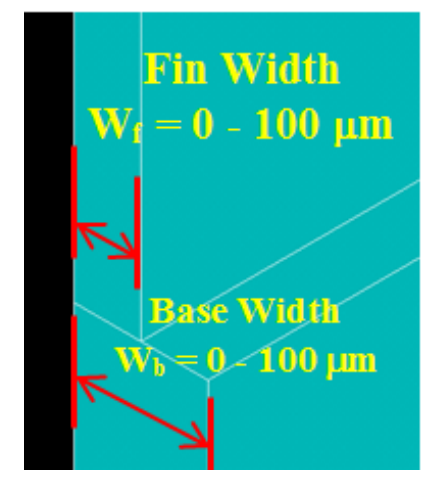

Figure 4.11: Microchannel Base and Fin Dimensions

\subsection{Material Properties}

The reliability study focusses on structural integration of a novel thermoelectric Mini-contact based cooling methodology with manifold-microchannel cooling directly on the back of a SiC chip. The base of the power electronics package is $\mathrm{GaN}$ electronics on $\mathrm{SiC}$ substrate. A non-linear stress analysis was performed to evaluate plastic strain at the various integration levels in the power electronics package. Later, inelastic strain was input to the failure model given by Engelmaier to predict Mean Time to Failure (MTTF) of the solder interconnection.

The Mini-contact structure is either an integrated $(\mathrm{SiC})$ or discrete (copper or diamond) version. The discrete Mini-contact is integrated with the $\mathrm{SiC}$ substrate with 
a SAC305 solder joint. Non-linear properties of SAC305 solder were input to the model. The thermo-electric cooler is a stack of AlN/Copper/SAC305 solder both on top and bottom of the thermo-electric element, which is made up of Bismuth Telluride $\left(\mathrm{Bi}_{2} \mathrm{Te}_{3}\right)$. The TE Cooler is integrated with Mini-contact using a thin layer of SAC305 solder joint. The properties of different materials listed above are presented as follows. [28 - 31, 47 - 49]

\subsubsection{Material Properties Data}

Table 4.1: Materials Properties Input to FEA Model, [28 - 31, 47 - 49]

\begin{tabular}{|c|c|c|c|c|c|}
\hline Materials & $\begin{array}{c}\text { Elastic } \\
\text { Modulus } \\
(\mathbf{E}) \\
\mathbf{G P a}\end{array}$ & $\begin{array}{c}\mathbf{C . T . E .} \\
(\boldsymbol{\alpha}) \\
\mathbf{p p m} / \mathbf{K}\end{array}$ & $\begin{array}{c}\text { Thermal } \\
\text { Conductivity } \\
(\mathbf{k}) \\
\mathbf{W m}^{-1} \mathbf{K}^{-\mathbf{1}}\end{array}$ & $\begin{array}{c}\text { Poisson's } \\
\text { Ratio } \\
(\mathbf{v})\end{array}$ & $\begin{array}{c}\text { Yield } \\
\text { Strength } \\
\left(\boldsymbol{\sigma}_{\mathbf{y}}\right) \\
\mathbf{G P a}\end{array}$ \\
\hline $\mathrm{SiC}(4 \mathrm{H})$ & 480 & $3.7-4.3$ & $\begin{array}{c}\text { function of } \\
\text { temperature }\end{array}$ & 0.175 & 0.129 \\
\hline $\mathrm{GaN}$ & 295 & 3.2 & 130 & 130 & - \\
\hline $\mathrm{SAC} 305$ & 90 & 24 & 65 & 0.24 & 0.05 \\
\hline Copper & 110 & 16.4 & 400 & 0.343 & $0.05-0.3$ \\
\hline $\mathrm{Diamond}$ & 1050 & $1-2$ & 1800 & 0.1 & $1-3$ \\
\hline $\mathrm{Bi}_{2} \mathrm{Te}_{3}$ & 436 & 17.8 & $1.5-2.4$ & 0.33 & - \\
\hline $\mathrm{AlN}$ & 330 & 4.5 & 250 & 0.24 & - \\
\hline
\end{tabular}

As mentioned above, the thermal conductivity of $\mathrm{SiC}$ is a function of temperature. There are two models available in literature, namely by research scientist Slack and Bergem. [48] The Slack model is a more conservative model and thus, for modeling purposes, use of Slack model was made. Temperature independent properties were used for Diamond. Figure 4.11 gives an idea about the variation in thermal conductivity of $\mathrm{SiC}$ as a function of temperature, as per Bergem's model and it was generated using Ansys. 


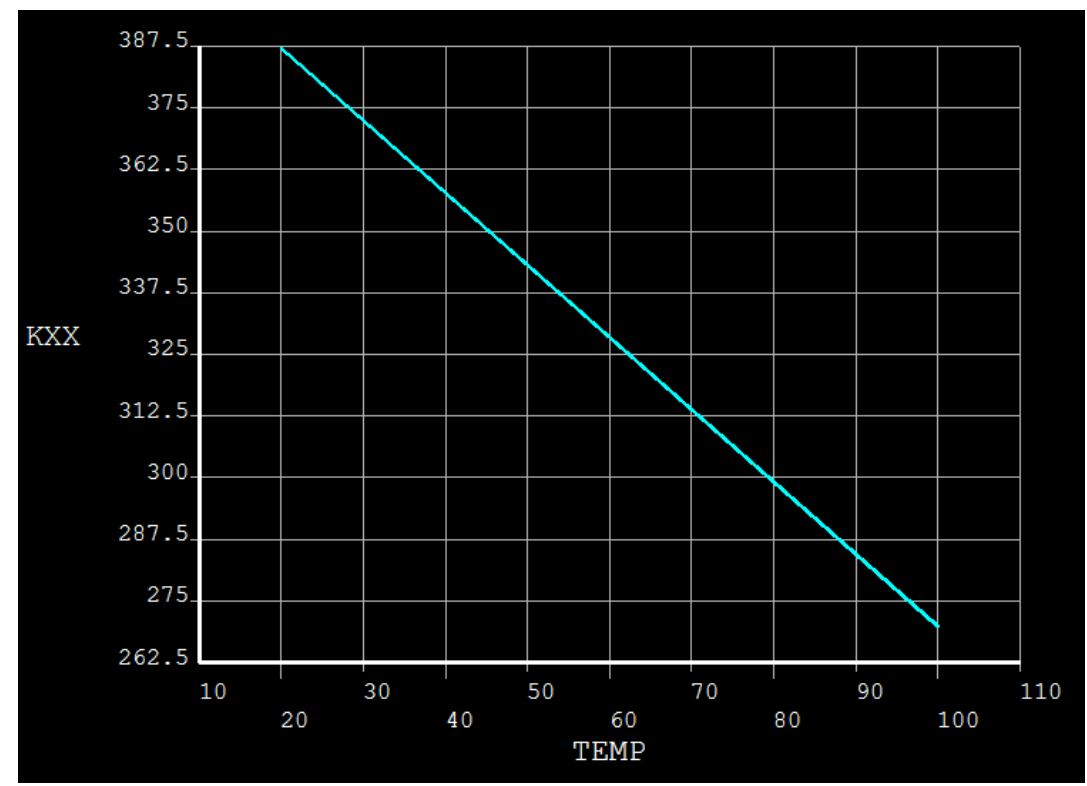

Figure 4.12: Temperature dependent SiC Thermal Conductivity

Stress vs strain properties of SAC305 solder are presented as below. These values were reported in research works by Hongtao Ma [30]. For a non-linear thermal mechanical stress analysis, input of plastic data is important in order to study plastic deformation in the material of interest.

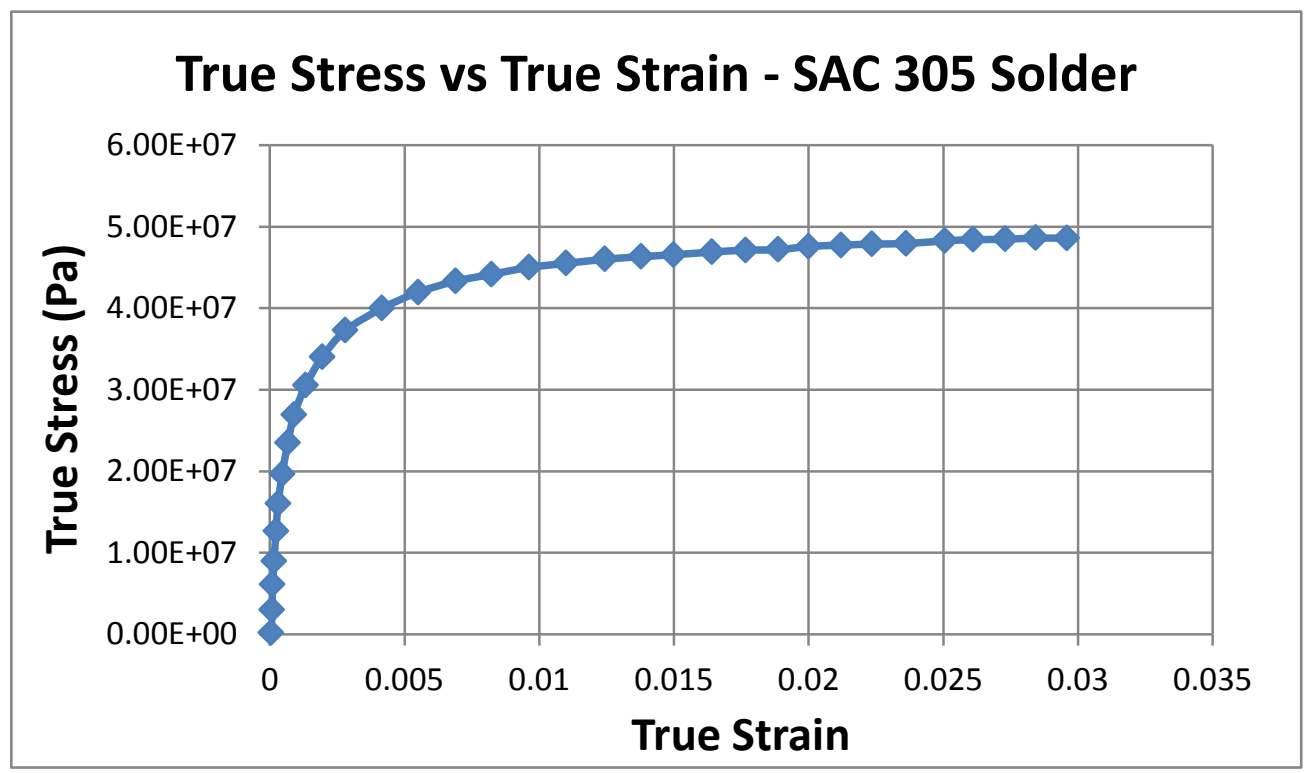

Figure 4.13: SAC305 True Stress vs True Strain Properties 
The material properties of Copper are presented as shown below. Temperature dependent $\left(20^{\circ} \mathrm{C}, 75^{\circ} \mathrm{C}, 125^{\circ} \mathrm{C}\right.$ and $\left.175^{\circ} \mathrm{C}\right)$ plastic data was input for Copper.

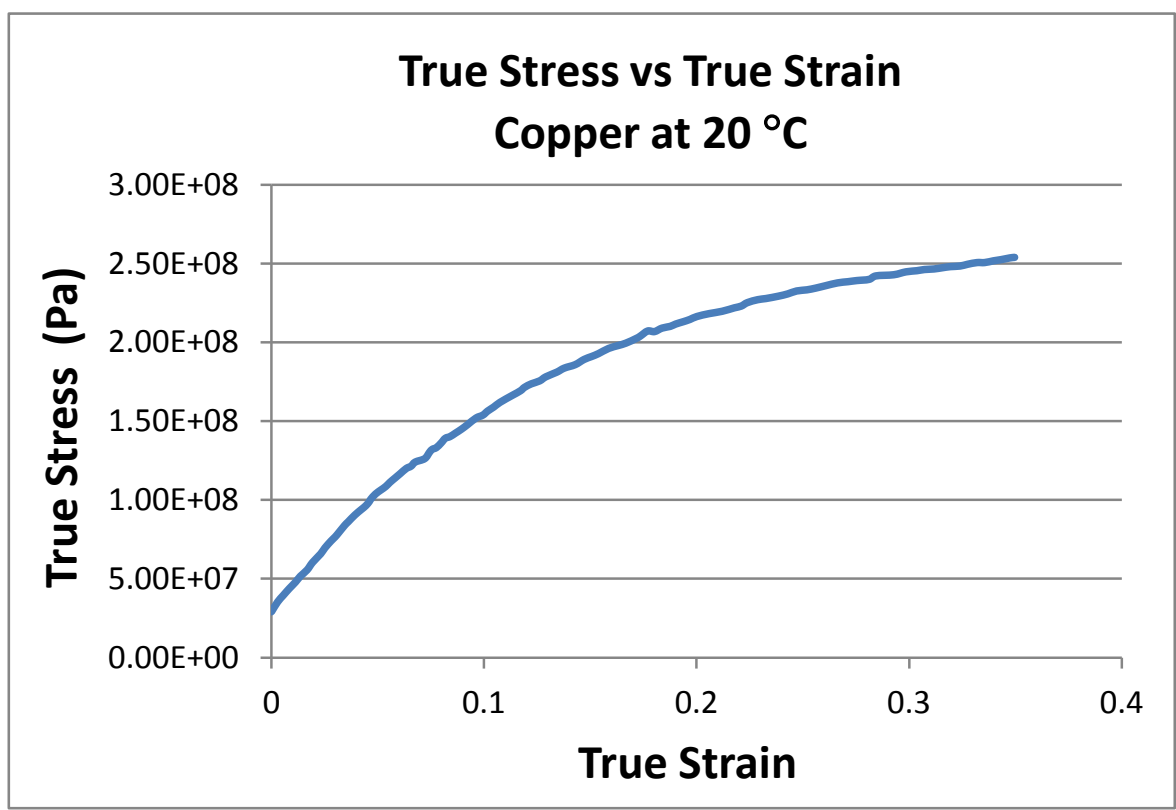

Figure 4.14: True Stress vs True Strain - Copper at $20^{\circ} \mathrm{C}$

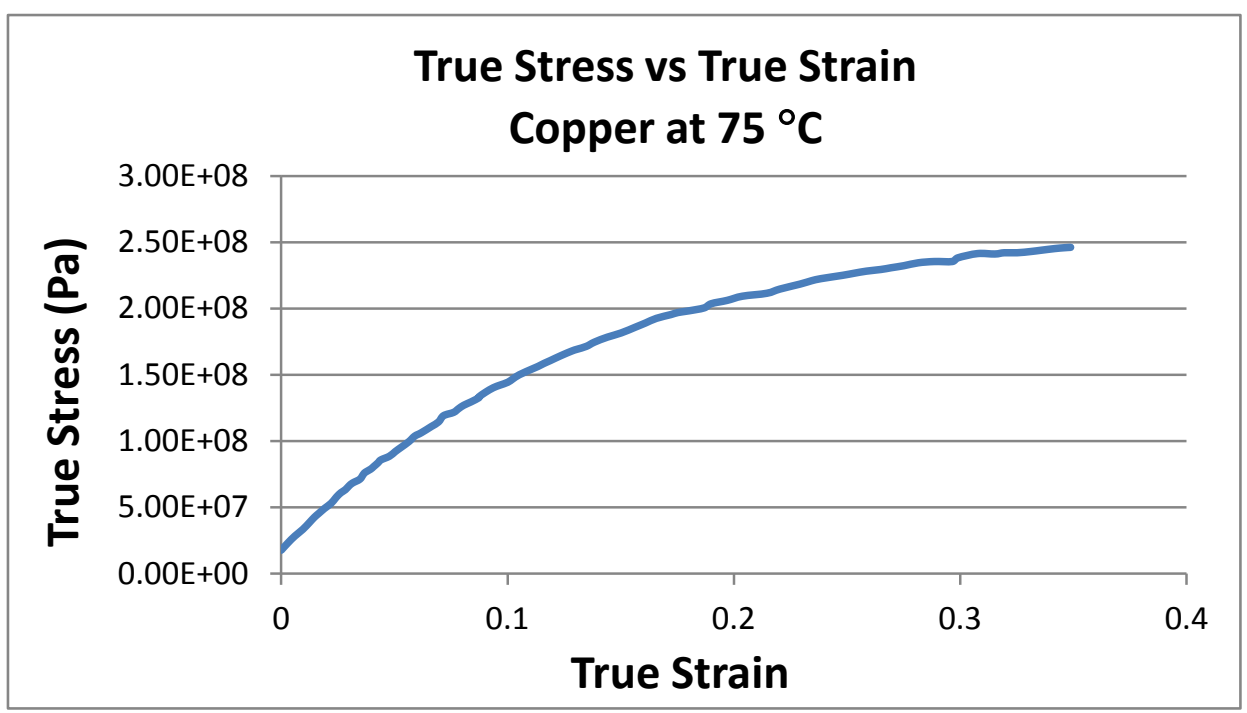

Figure 4.15: True Stress vs True Strain - Copper at $75^{\circ} \mathrm{C}$ 


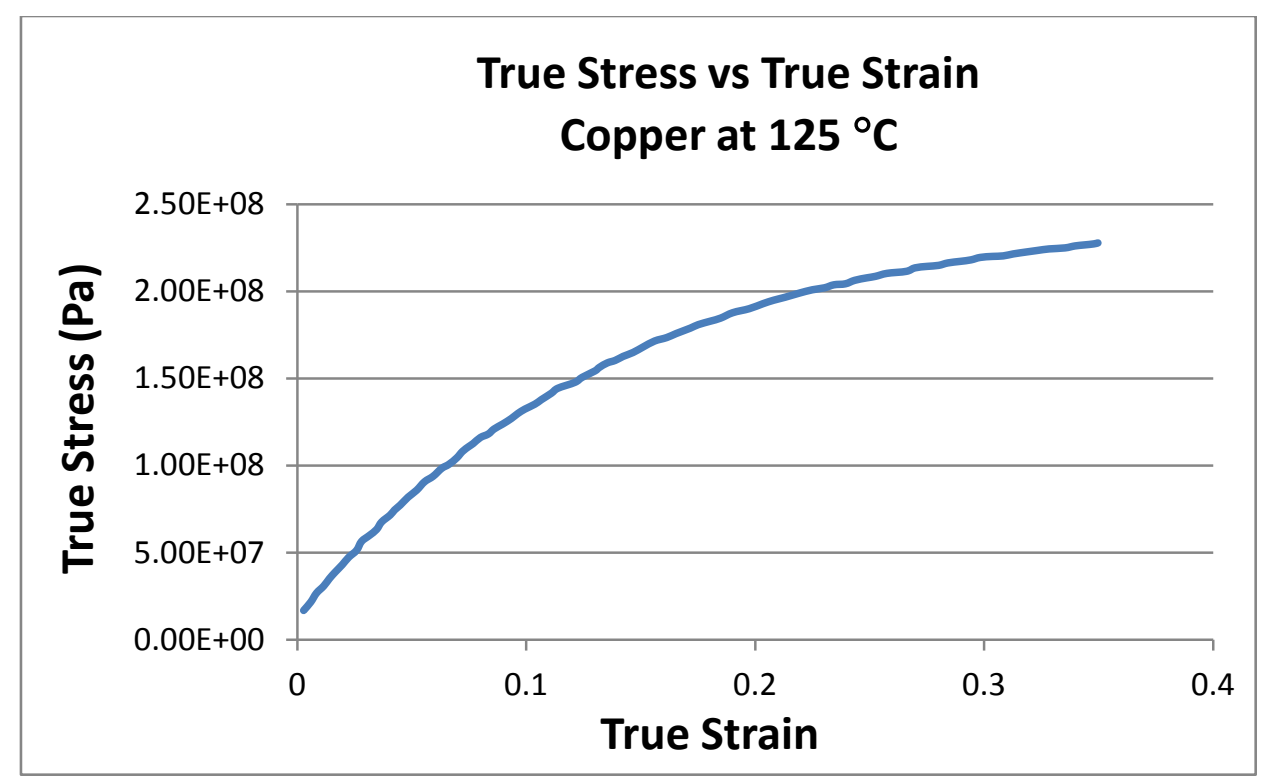

Figure 4.16: True Stress vs True Strain - Copper at $125^{\circ} \mathrm{C}$

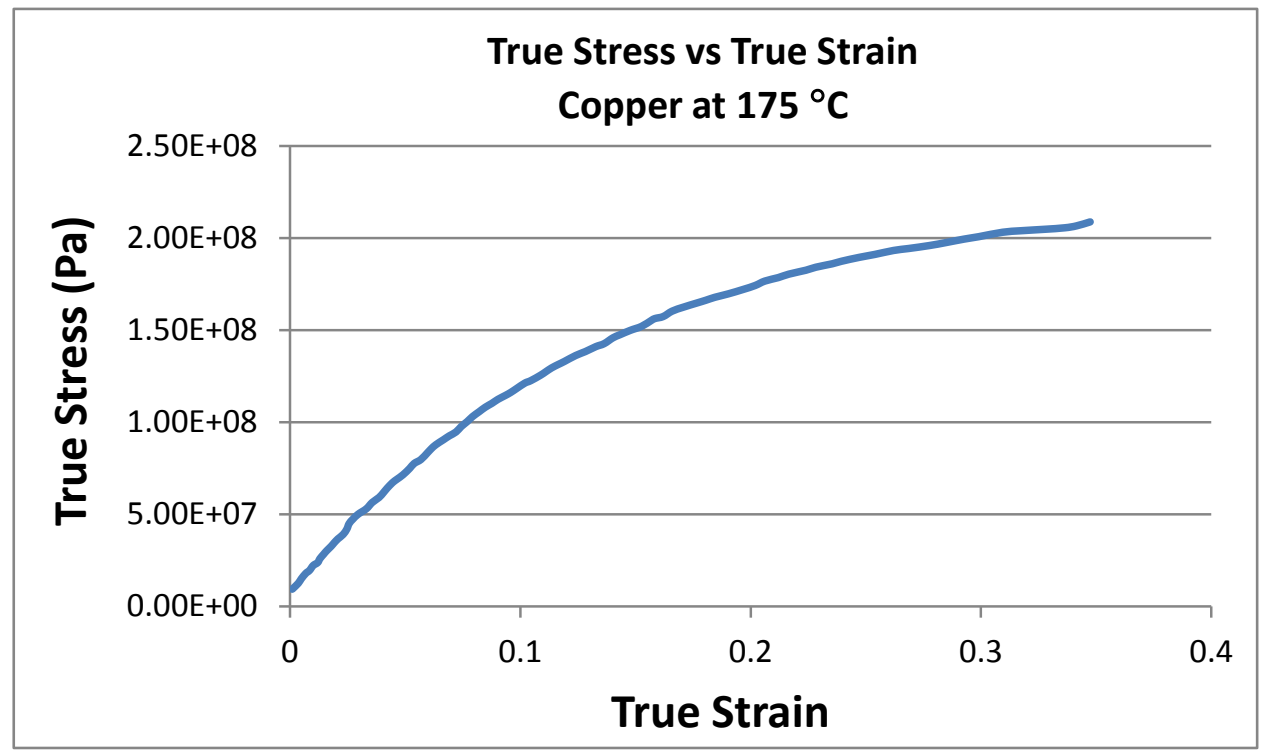

Figure 4.17: True Stress vs True Strain - Copper at $175^{\circ} \mathrm{C}$

Material references [28 - 31, 47 - 49] 


\subsection{Element Selection and Mesh Generation}

For solution to any Finite Element (FE) analysis problem, factors contributing to solution time and accuracy of analysis results include selection of appropriate element type and right mesh density in the critical areas of concern in the CAD model. For thermo-electric based Mini-contact cooling, hot-spot temperature and various interconnection levels were considered for selection of appropriate element size. An attempt was made to ensure a structured grid based on use of free and mapped meshing options. Control of mesh sizing was also equally important for Manifold-Microchannel based cooling. Mesh sizing for the two models is explained further in detail in the following sections.

\subsubsection{Mini-contact based Thermo-electric Cooling}

For thermo-mechanical stress analysis in the thermo-electric Mini-contact cooling region, use of Sold 226 element was made. It is a higher order 3-D 20 node element which has thermo-electric, thermal and structural mechanics capability.

The focus of this thermo-mechanical stress analysis was studying failure in the structure due to C.T.E. mismatch of various materials used at different levels of integration in the power electronics package, specifically in thermo-electric based Mini-contact cooling region. As it can be inferred from figure 4.17 below, the CAD model includes the TE Cooler structure sitting at top of the hot-spot region, in addition to the micro-channel cooling region (without fins). Thermo-electric cooling is supposed to have an effect on die temperature in a $1 \mathrm{~mm}$ radius region surrounding 
the Mini-contact structure. The remainder of package (die) region is controlled by micro-fluidics cooling.

The mesh density study is based on hot-spot temperature. The region outside the Mini-contact domain, i.e. microfluidics, contributes to majority of package volume. Consequently, the size of elements in this region will affect the solution time. However, one needs to realize that a smaller element size in microfluidics domain will cost a lot of computer space and solution time. In order to neglect this effect and achieve solution in reasonable time, a comparatively coarser mesh was set in the microfluidics region, which still provided reasonable hot-spot temperature and the uniform temperature distribution across the package. Keeping the size of element edge length fixed as $700 \mu \mathrm{m}$ in the microfluidics region, focus was laid on finer mesh in the Mini-contact region. Also, a finer mesh in micro-fluidics region will result in drop of temperature in that region. A combination of sweep and free meshing was applied in the Mini-contact region. The following curve gives the change in hot-spot temperature as a function of the number of equations generated. For application of mesh sizing, the package was broken down into different regions, where the reduction of element size was performed until it made no change in the temperature of the hotspot. The different regions with element (edge length) sizes are listed in Table 4.1. 


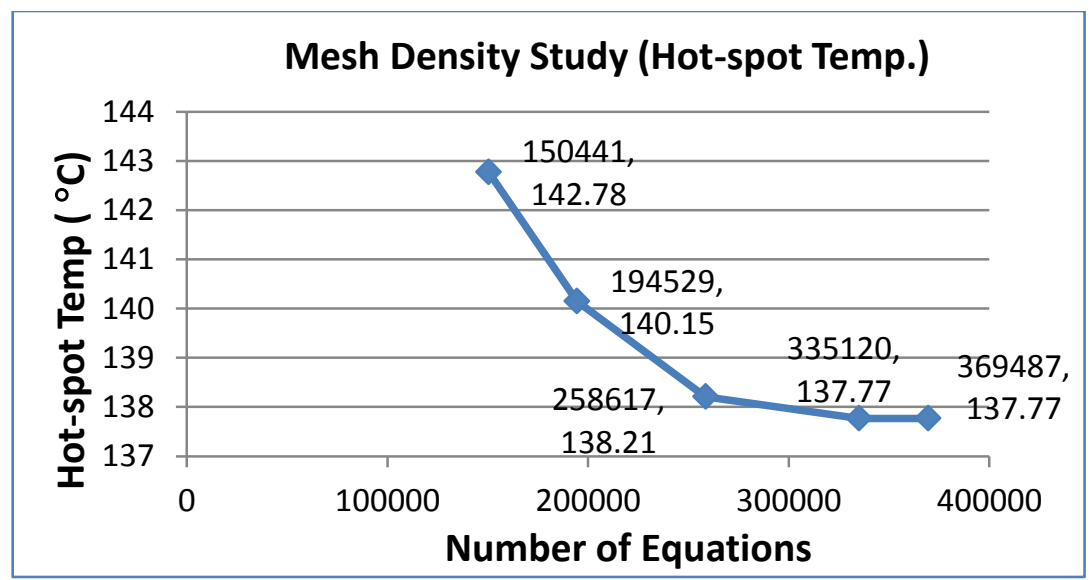

Figure 4.18: Mesh Density Study (Hot-spot Temperature)

Table 4.2: Application of Mesh Sizing Options

\begin{tabular}{|c|c|c|c|}
\hline $\begin{array}{c}\text { Region } \\
\text { Representation }\end{array}$ & Package Region & Dimensions & $\begin{array}{c}\text { Line Size or } \\
\text { Element Edge } \\
\text { Length } \\
\end{array}$ \\
\hline \# A & Hot-spot $(x, y)$ & $100 \mu \mathrm{m}$ x $100 \mu \mathrm{m}$ & $\begin{array}{c}\text { Line sizing: } 15 \\
\text { parts }\end{array}$ \\
\hline \# B & $\begin{array}{l}\text { Outside hot-spot } \\
\text { region (Mini-contact } \\
\text { domain) }\end{array}$ & $50 \mu \mathrm{m}$ & Line sizing: 5 parts \\
\hline \# C & $\begin{array}{l}\text { Mini-contact } \\
\text { surrounding region - } \\
\text { SiC substrate }\end{array}$ & $850 \mu \mathrm{m}$ & $\begin{array}{l}\text { Line sizing: } 10 \\
\text { parts }\end{array}$ \\
\hline \# D & SiC substrate $(\mathrm{z})$ & $100 \mu \mathrm{m}$ & $\begin{array}{c}\text { Line sizing: } 10 \\
\text { parts }\end{array}$ \\
\hline$\# \mathrm{E}$ & GaN region $(\mathrm{z})$ & $3 \mu \mathrm{m}$ & Line sizing: 2 parts \\
\hline \# F & Solder joint $(x, y)$ & $600 \mu \mathrm{m}$ & $\begin{array}{l}\text { Line sizing: } 10 \\
\text { parts }\end{array}$ \\
\hline$\# \mathrm{G}$ & Solder joint $(\mathrm{z})$ & $20 \mu \mathrm{m}$ & Line sizing: 5 parts \\
\hline$\# \mathrm{H}$ & Mini-contact region & $\begin{array}{c}300 \mu \mathrm{m} \text { (top base } \\
\text { width) x } 150 \mu \mathrm{m} \\
\text { (bottom base width) } \\
\text { x } 600 \mu \mathrm{m} \text { (ht.) }\end{array}$ & $\begin{array}{l}\text { Element edge } \\
\quad \text { length } \\
=75 \mu \mathrm{m}\end{array}$ \\
\hline \# I & TE Cooler Headers & $\begin{array}{c}300 \mu \mathrm{m} \text { (top base } \\
\text { width) x } 300 \mu \mathrm{m} \\
\text { (top base length) } \mathrm{x} \\
100 \mu \mathrm{m} \text { (ht.) }\end{array}$ & $\begin{array}{l}\text { Element edge } \\
\quad \text { length } \\
=100 \mu \mathrm{m}\end{array}$ \\
\hline \# J & $\begin{array}{c}\text { Metallized Copper } \\
\text { Straps }\end{array}$ & $10-20 \mu \mathrm{m}$ & $\begin{array}{l}\text { Element edge } \\
\text { length } \\
=20 \mu \mathrm{m}\end{array}$ \\
\hline
\end{tabular}




\begin{tabular}{|c|c|c|c|}
\hline \# K & $\begin{array}{c}\text { Solder joint } \\
\text { interconnecting } \\
\text { Copper straps with } \\
\text { thermoelectric } \\
\text { elements }\end{array}$ & $10-20 \mu \mathrm{m}$ & $\begin{array}{c}\text { Element edge } \\
\text { length } \\
=20 \mu \mathrm{m}\end{array}$ \\
\hline \# & $\begin{array}{c}\text { Thermoelectric } \\
\text { Elements in TE } \\
\text { Cooler }\end{array}$ & $40-60 \mu \mathrm{m}$ & $\begin{array}{c}\text { Element edge } \\
\text { length } \\
=15 \mu \mathrm{m}\end{array}$ \\
\hline \# & SiC substrate & $\begin{array}{c}10 \mathrm{~mm} \times 10 \mathrm{~mm} \times \\
100 \mu \mathrm{m}\end{array}$ & $\begin{array}{c}\text { Element edge } \\
\text { length } \\
=700 \mu \mathrm{m}\end{array}$ \\
\hline \multicolumn{2}{|c|}{ Number of equations $\approx 860,000 ;$ Solve Time: $3-4$ hours } \\
\hline
\end{tabular}

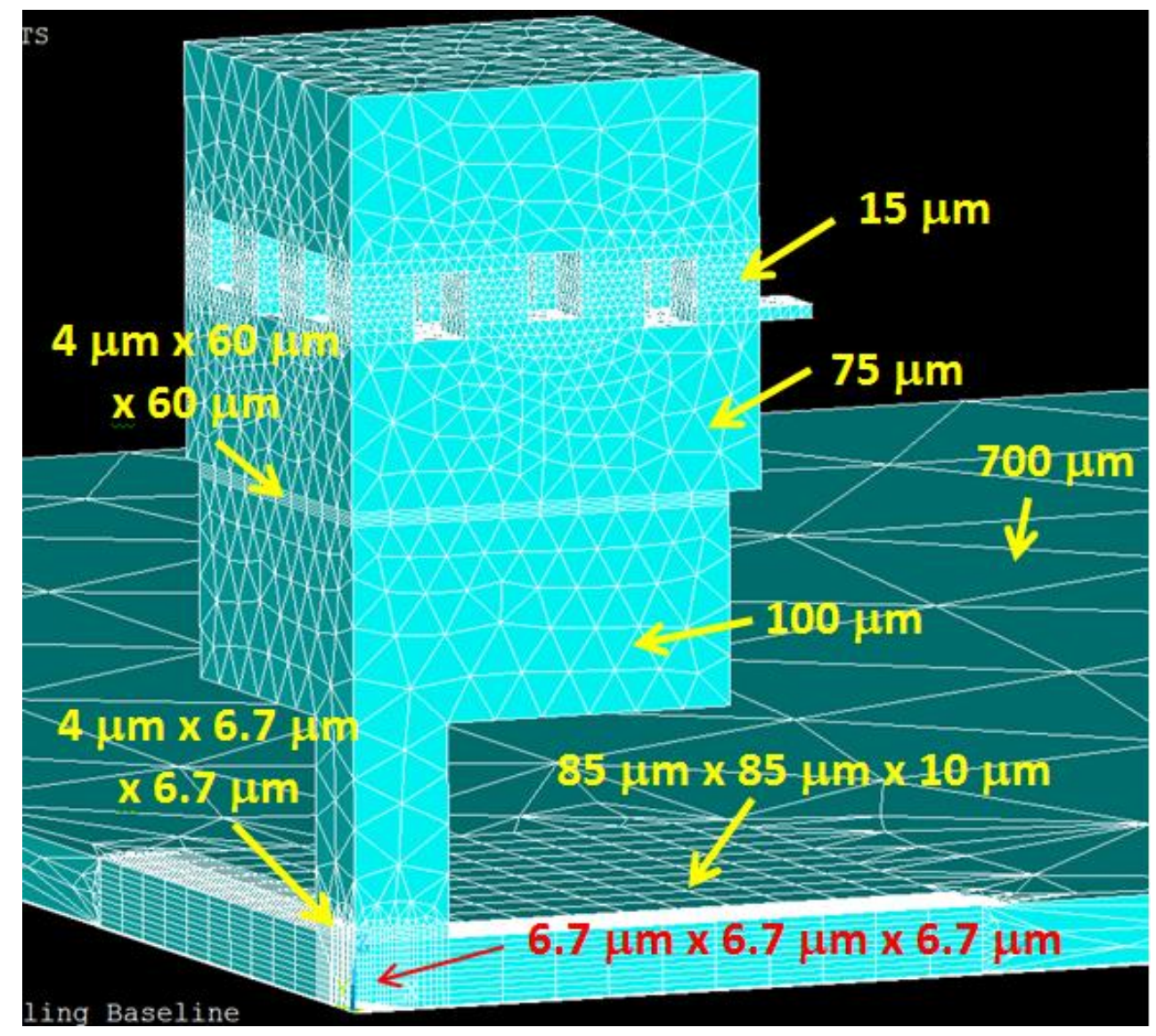

Figure 4.19: Mesh Sizing across the Package

The sizing of elements in individual regions will be presented below. 


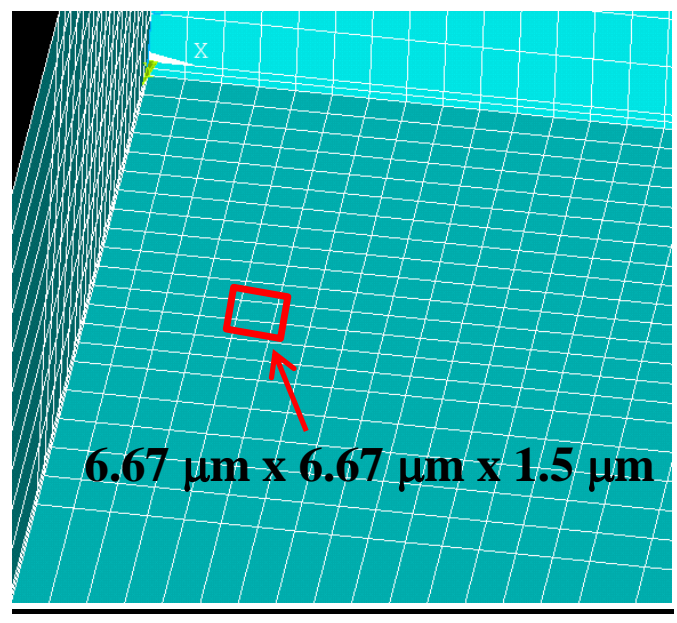

Figure 4.20: Hot-spot Region Element Sizing

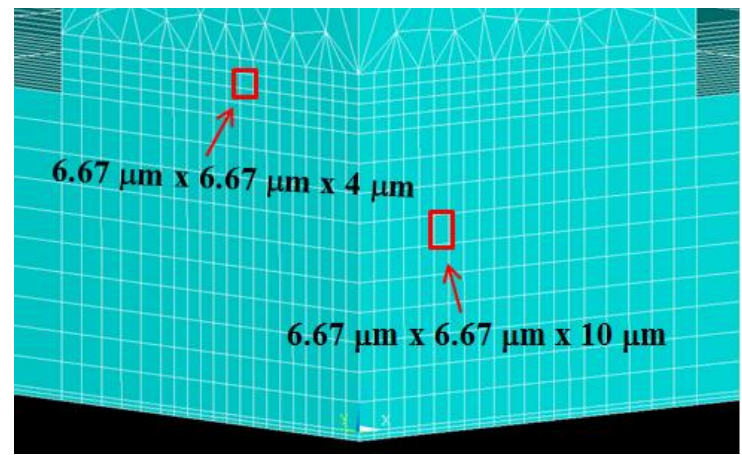

Figure 4.21: SiC Substrate Region (Mini-contact domain) Element Sizing

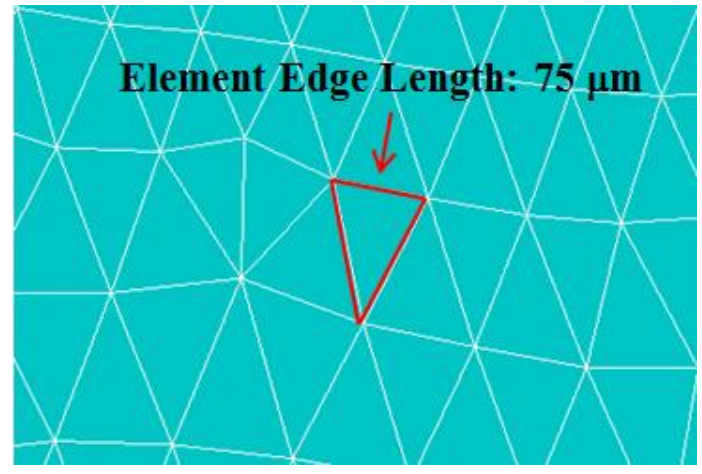

Figure 4.22: Mini-contact Region Mesh Sizing 


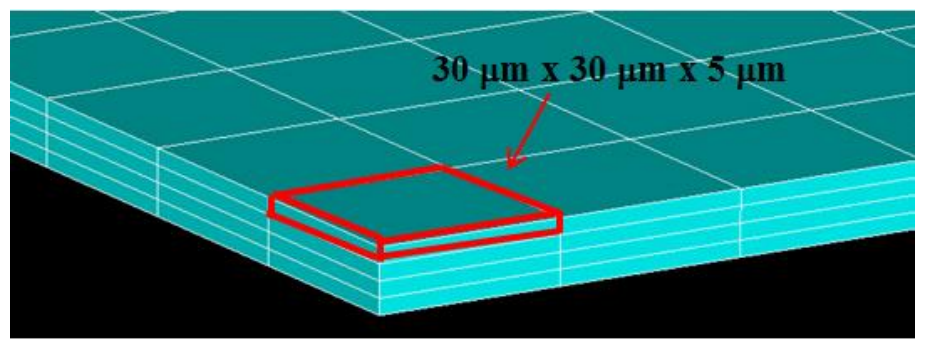

Figure 4.23: Solder Joint TE Cooler and Mini-contact Mesh Sizing

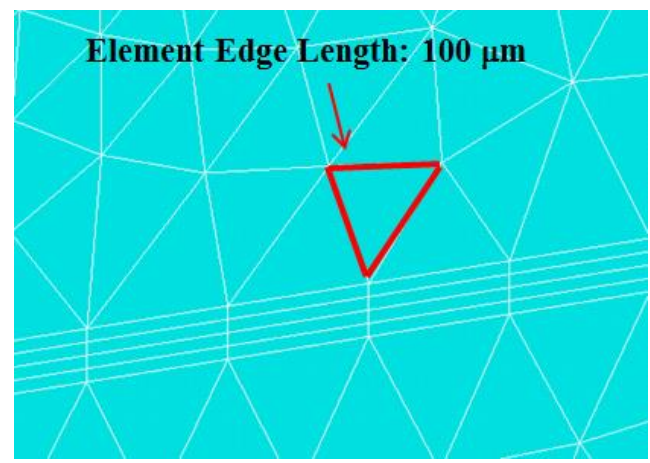

Figure 4.24: TE Cooler Header Element Edge Length

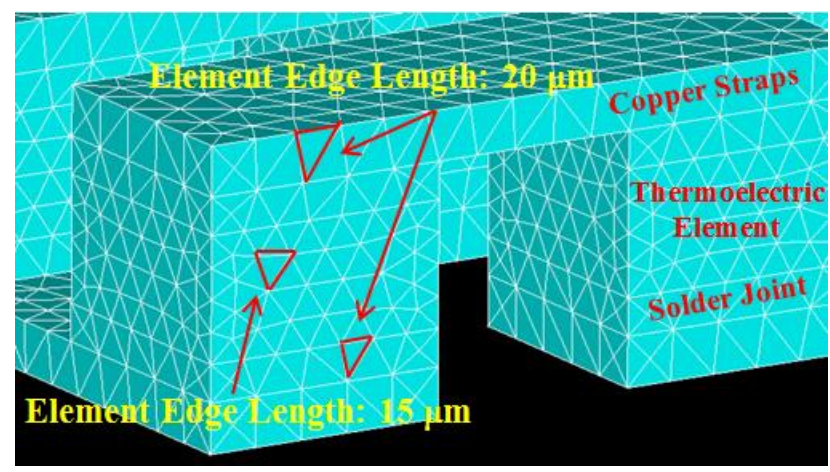

Figure 4.25: Element Edge Length - TE Elements, Solder Joint and Copper Straps

\subsubsection{Micro-channel Cooling}

For thermo-mechanical stress analysis of the micro-fin assembly, a

combination of a lower order eight node purely thermal element and its mechanical counterpart was made. The selection of elements was based on the application of tabular convection boundary condition. The convection heat transfer coefficient data was available for a set of $\mathrm{x}, \mathrm{y}$ co-ordinates in the micro-fin cooling space regime. 
With eight node element, one can take advantage of the tabular boundary condition available with Ansys Mechanical. For thermal analysis, Solid 70 element was used and for mechanical analysis, it was replaced by Solid 45 element. As shown below in figure 4.25, a finite element model of unit cell model was used for FE analysis.

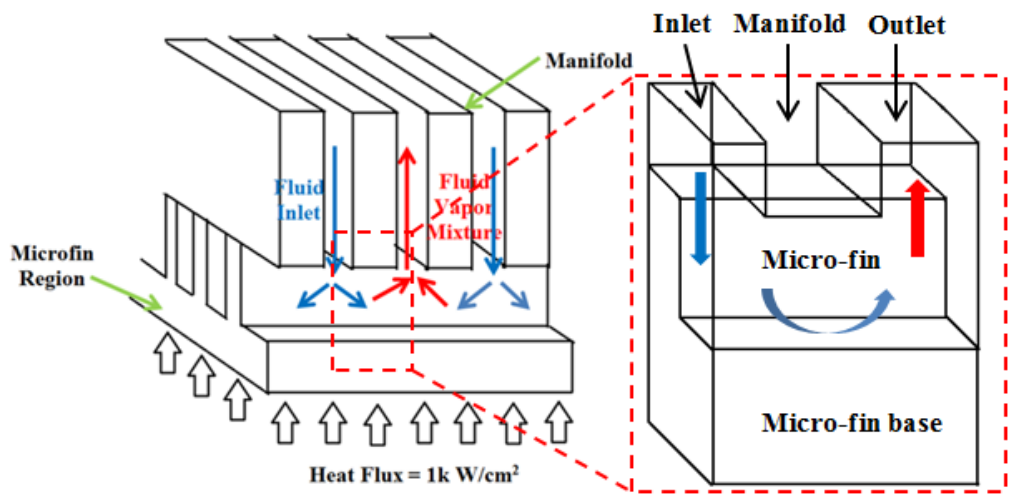

Figure 4.26: Unit Cell Model - Microfluidics Cooling [38]
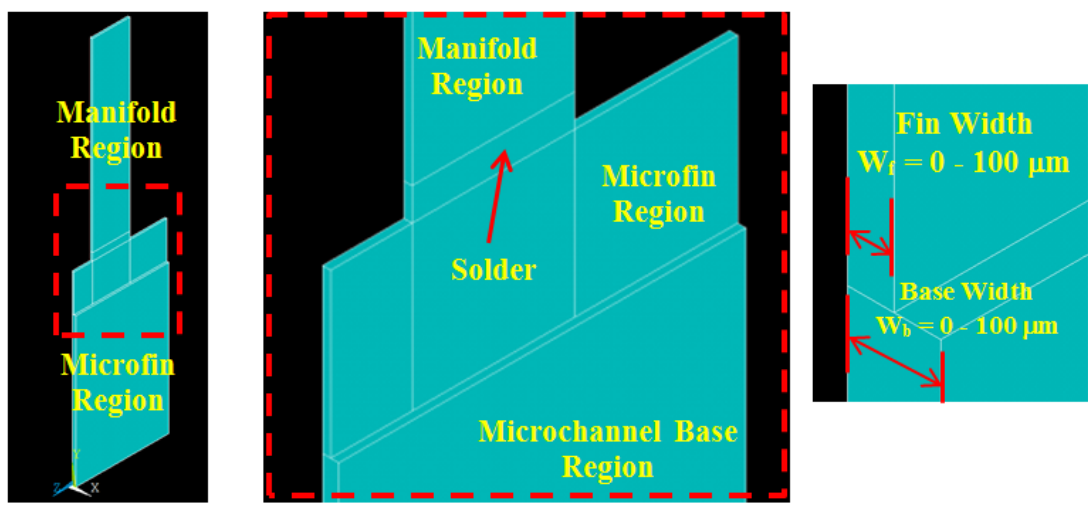

Figure 4.27: CAD model - Unit Cell Model - Microfluidics

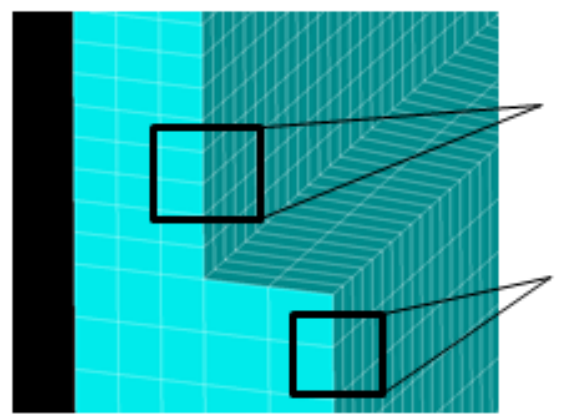

Microfin Region $\left(\mathrm{X}^{*} \mathrm{Y}^{*} \mathrm{Z}\right)$

$1 \mathrm{um} \times 1 \mathrm{um} \times 1.67 \mathrm{um}$

Microfin Base Region

$1 \mathrm{um} \times 1 \mathrm{um} \times 2.5 \mathrm{um}$

Figure 4.28: Mesh Sizing - Microfluidics 
As shown above in figure 4.27, an element size of 1 um x 1 um x 1.67 um was applied for the CAD model in the micro-fin region. The small size of element helps with interpolation of the convection heat transfer coefficient data available for a set of $\mathrm{x}$ and $\mathrm{y}$ co-ordinates across the micro-fin region. [38]

\section{$\underline{4.4 \text { Boundary Conditions }}$}

This section gives details on the thermal and mechanical boundary conditions used for the two different FE models (viz. Mini-contact based thermo-electric cooling and Microfluidics cooling). Thermal and mechanical boundary conditions were applied with the help of ANSYS command language. The boundary conditions are explained in further detail in the appendix at the end of the document. The boundary conditions are explained separately for the two models in the following sections.

\subsubsection{Mini-contact based Thermo-electric Cooling}

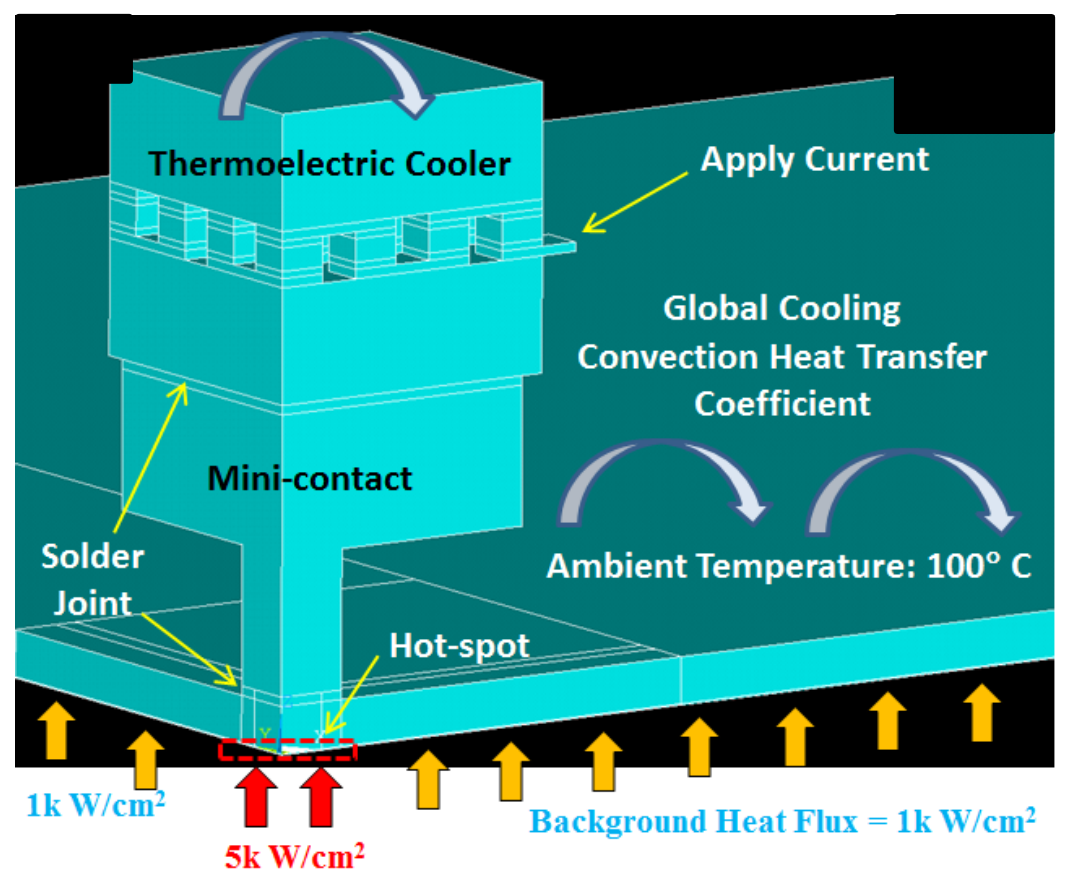

Figure 4.29: Application of Thermal Boundary Conditions 
The figure above gives an overview of thermal boundary conditions applied to the package. A heat flux of $5 \mathrm{k} \mathrm{W} / \mathrm{cm}^{2}$ is applied at the hot-spot and a heat flux of $1 \mathrm{k}$ $\mathrm{W} / \mathrm{cm}^{2}$ is applied as the background heat flux for GaN on SiC. The hot-spot dimensions are $200 \mu \mathrm{m} \times 200 \mu \mathrm{m}$ and the heat is dissipated by concentrating a cooling flux of $200 \mathrm{~W} / \mathrm{cm}^{2}-250 \mathrm{~W} / \mathrm{cm}^{2}$ supplied via the thermo-electric cooler at the tip of the Mini-contact.

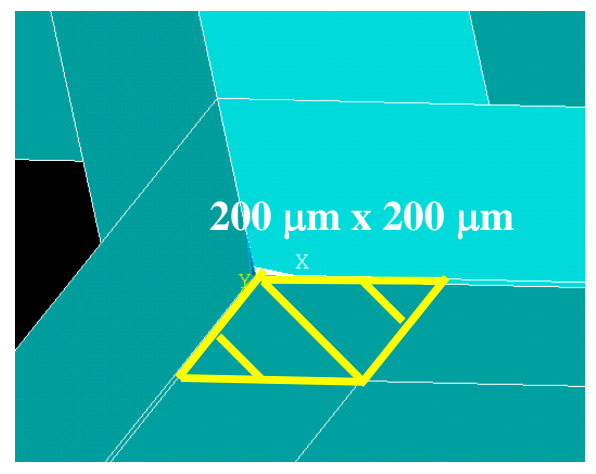

Figure 4.30: Hot-spot Heat Flux of 5k W/cm² (Quarter Symmetric Model)

The background heat flux is mitigated through a two phase coolant. A combination of different fluids have been proposed for this research study, which have saturation temperatures in the range of $100^{\circ} \mathrm{C}-150^{\circ} \mathrm{C}$. Selection of coolant is out of scope of this study. However, water (saturation: $100^{\circ} \mathrm{C}$ ) has been chosen as one of the probable coolants. A convection heat transfer coefficient of $364 \mathrm{k} \mathrm{W} / \mathrm{cm}^{2}$ was used in the FE model.

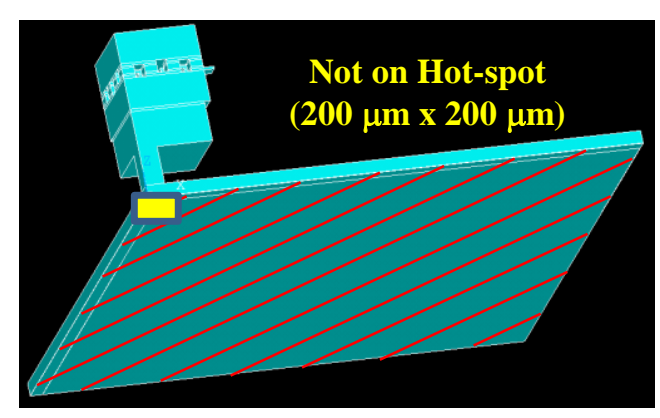

Figure 4.31: Background Heat Flux of $1 \mathrm{k} \mathrm{W} / \mathrm{cm}^{2}$ 


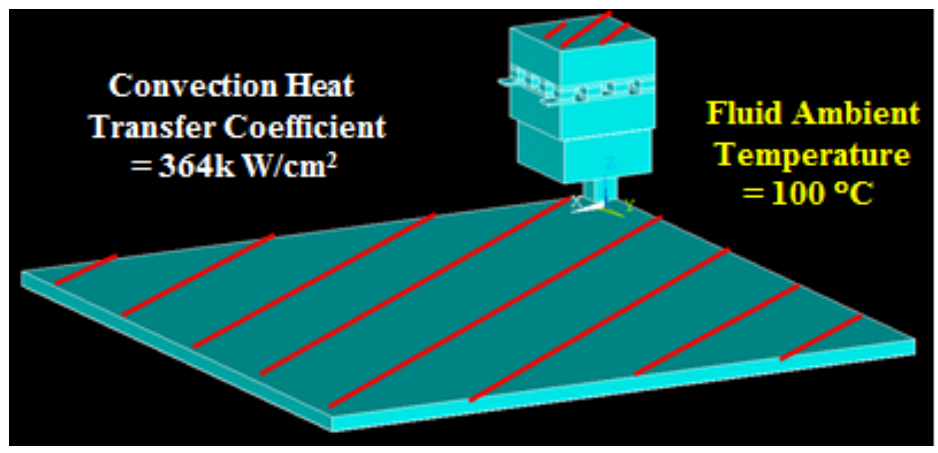

Figure 4.32: Application of Global Heat Transfer Convection Coefficient

In addition to the heat fluxes and convection heat transfer coefficient, there are thermo-electric boundary conditions, which aim to provide a cooling flux in the range of $200 \mathrm{~W} / \mathrm{cm}^{2}-250 \mathrm{~W} / \mathrm{cm}^{2}$ at the tip of Mini-contact. These boundary conditions are further explained as below.

Table 4.3: Parameters set to the TE Cooler

\begin{tabular}{|c|c|}
\hline Parameter & Magnitude \\
\hline Initial Current & $1.25 \mathrm{~A}$ \\
\hline Final Current & $1.25 \mathrm{~A}$ \\
\hline Current Increment & $10^{10} \mathrm{ohm}-\mathrm{m}$ \\
\hline $\begin{array}{c}\text { Insulating Cooler Resistivity } \\
\text { Contact resistance volume } \\
\text { thickness }\end{array}$ & $15 \mu \mathrm{m}$ \\
\hline $\begin{array}{c}\text { Contact resistance volume } \\
\text { Contact Resistance }\end{array}$ & $5.5 \times 10^{-11} \mathrm{ohm}-\mathrm{m}^{2}$ \\
\hline $\begin{array}{c}\text { Contact Resistance Volume } \\
\text { Resistivity }\end{array}$ & $3.64 \mathrm{e}-6 \mathrm{ohm}-\mathrm{m}$ \\
\hline $\begin{array}{c}\text { Header electrical resistivity } \\
10^{10} \mathrm{ohm}-\mathrm{m}\end{array}$ & $10 \times 10^{-6} \mathrm{ohm}-\mathrm{m}$ \\
\hline Electrical resistivity (P, N) & $(-) 200 \times 10^{-6} \mathrm{Volt} / \mathrm{K}$ \\
\hline Seebeck Coefficient (P) & $200 \times 10^{-6} \mathrm{Volt} / \mathrm{K}$ \\
\hline Seebeck Coefficient (N) & $1.7 \times 10^{-8} \mathrm{ohm}-\mathrm{m}$ \\
\hline Copper resistivity & \\
\hline
\end{tabular}


The thermo-electric cooler consists of headers (AIN), metallized Copper on headers $(\mathrm{AlN})$ and thermo-electric elements $\left(\mathrm{Bi}_{2} \mathrm{Te}_{3}\right)$, which are interconnected to the Copper via a $15 \mu \mathrm{m}$ thick solder joint. From a thermal standpoint, the input of material properties to the FE model for above materials includes resistivity. Additionally for themo-electric elements, we can input the seebeck coefficient. The resistivity values for headers, Copper on headers and thermo-electric elements are input directly to the model. However, the resistivity of the interface resistance volume (assumed as a solder joint of $15 \mu \mathrm{m}$ in mechanical) is calculated based on an assumed thermal contact resistance of $5.5 \times 10^{-11}\left({ }^{\circ} \mathrm{C} / \mathrm{W}\right)-\mathrm{m}^{2}$ or ohm $-\mathrm{m}^{2}$. [32] The resistivity and thermal conductivity of the contact resistance volume can be calculated as shown below.

Contact resistance $\left(\mathrm{R}_{\text {contact }}\right)$ is defined as the resistance per unit area. It can be written mathematically in terms of thermal resistance $\left(\mathrm{R}_{\text {conductivity }}\right)$ and area $(A)$ as follows.

$$
\begin{gathered}
R_{\text {contact }}=R_{\text {conductivity }} * A \\
R_{\text {conductivity }}=\frac{L}{K_{t h} * A}=\frac{\rho * L}{A}
\end{gathered}
$$

In above equations; $\mathrm{L}$ is thickness of contact volume, $\mathrm{K}_{\mathrm{th}}$ is thermal conductivity and $\rho$ is thermal resistivity. Substituting above values in the equation of contact resistance, we can calculate resistivity and conductivity of the interface resistance volume. They are expressed mathematically as follows.

$$
\begin{gathered}
\rho=\frac{R_{\text {contact }}}{L} \\
K_{\text {th }}=\frac{L}{R_{\text {contact }}}
\end{gathered}
$$


A resistivity of $3.67 \times 10^{-6} \mathrm{ohm}-\mathrm{m}$ was input to the FE model for the interface resistance volume for a contact resistance of $5.5 \times 10^{-11} \mathrm{ohm}-\mathrm{m}^{2}$.

One of the primary assumptions is that the complete package is a flip-chip bonded assembly with the printed circuit board on the active side of the $\mathrm{GaN}$ on $\mathrm{SiC}$ substrate. This assumption is explained schematically in the figure 4.32.

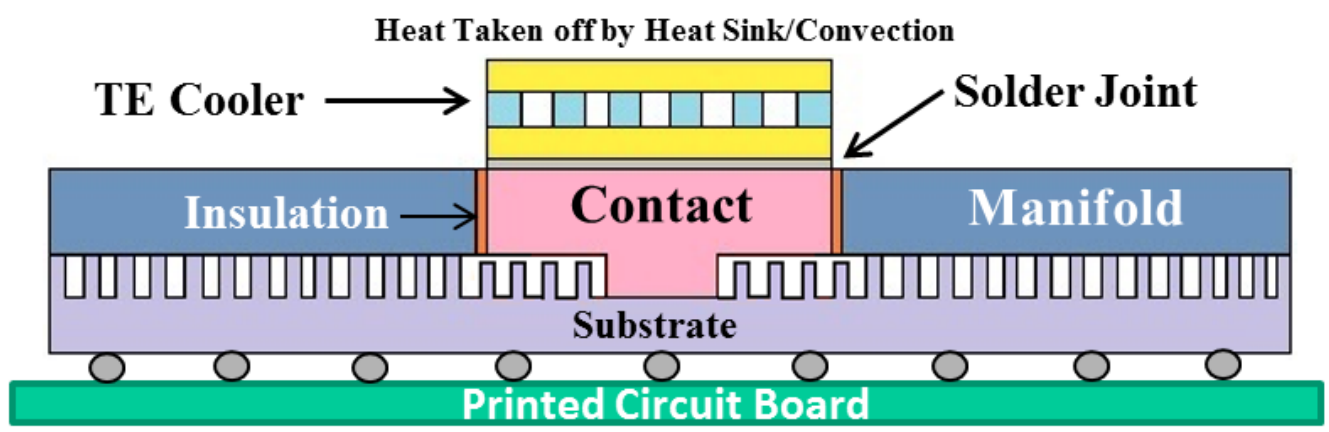

Figure 4.33: Flip Chip Package Assembly

The rationale behind flip-chip packaging is that it's a commonly used packaging methodology applied in electronics industry and it permits heat sinking from the back of the die. In terms of thermo-mechanical FE modeling, this means a displacement constraint equal to zero in $x-y-z$ directions at the bottom of the $\mathrm{GaN}$ on SiC substrate. This boundary condition is shown with the help of following schematic.

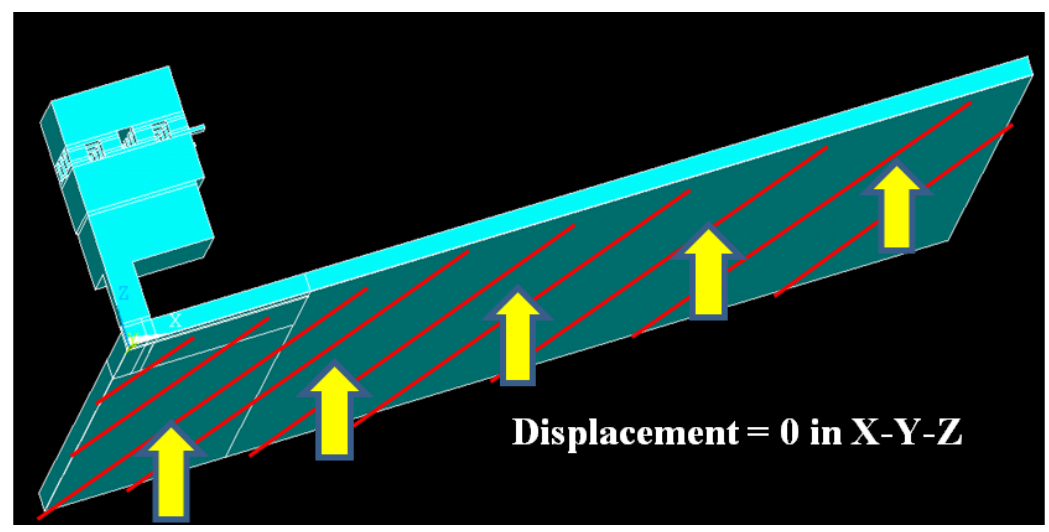

Figure 4.34: Package constrained at bottom of GaN on SiC substrate 
Other assumptions in the FE model include that there is a perfect adhesion between different volumes (all glue condition) at various levels across the package. Furthermore, the interface resistance volume acts as a solder joint interconnection.

The other boundary conditions include the symmetry boundary conditions on the $\mathrm{X}$ and $\mathrm{Y}$ faces of the power electronics package. As mentioned earlier, the advantage with use of a quarter symmetric CAD model is that it helps in reduction of the number of equations and consequently in solve time. These displacement boundary conditions are shown as follows.

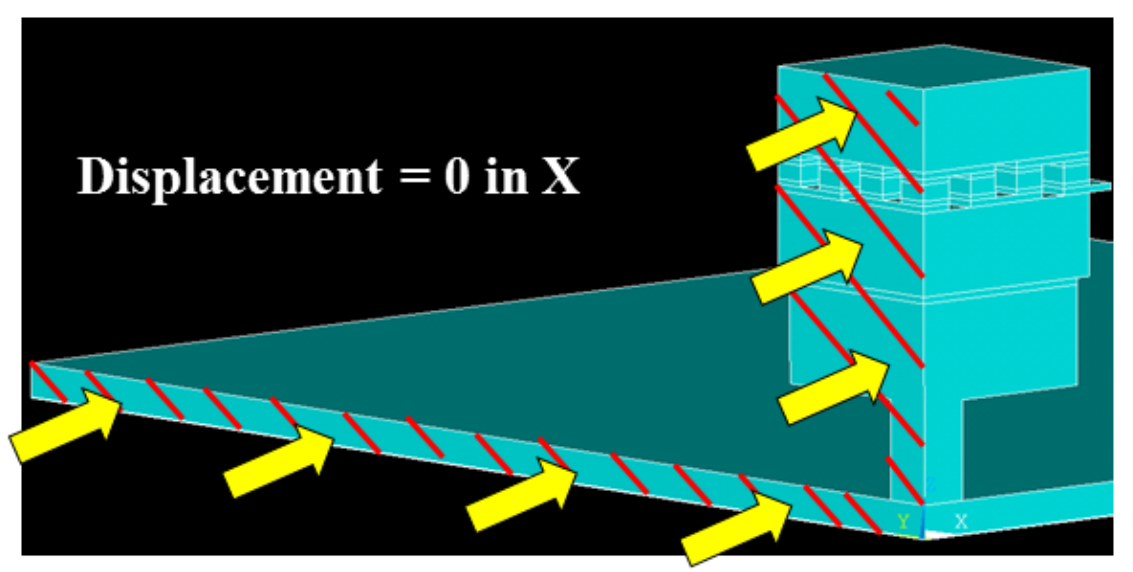

Figure 4.35: Quarter Symmetric Boundary Condition on X face

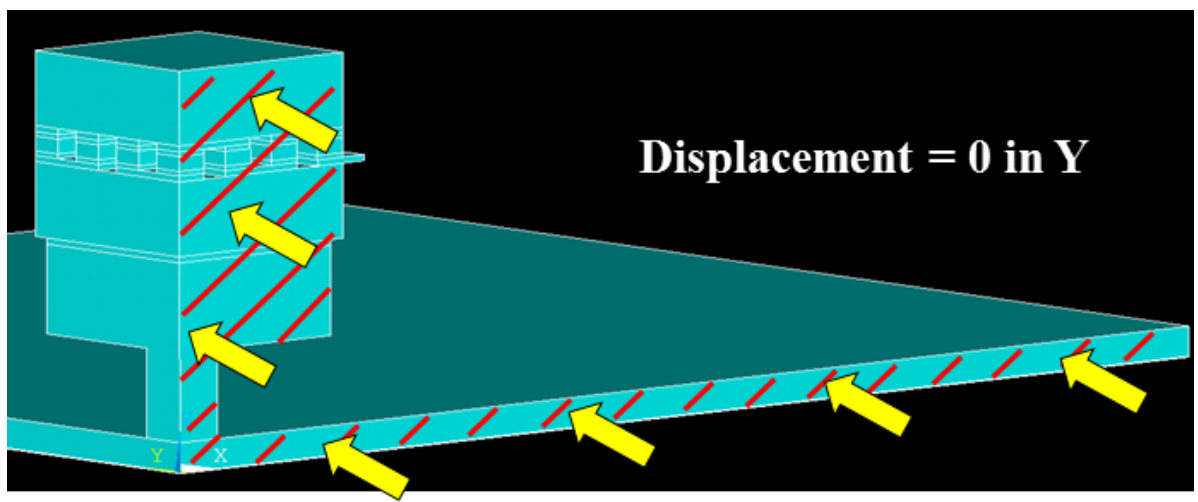

Figure 4.36: Quarter Symmetric Boundary Condition on Y face 


\subsubsection{Manifold Microchannel Cooling}

For stress analysis in the manifold microchannel region, a unit cell CAD model, as explained previously, was used for thermo-mechanical stress analysis. The thermal and mechanical boundary conditions will now be explained with the help of following schematics. [38] There are two types of symmetry boundary conditions used for this analysis. One is simply setting displacement $=0$ on the face perpendicular to the direction of the repeating unit cell. This boundary condition is based on the fact that the complete package is enclosed in a fixture. The second method of displacement boundary condition is use of planar symmetry conditions, which allows the package to be free. The real condition is a situation in between the above two boundary conditions. These boundary conditions are explained as follows.

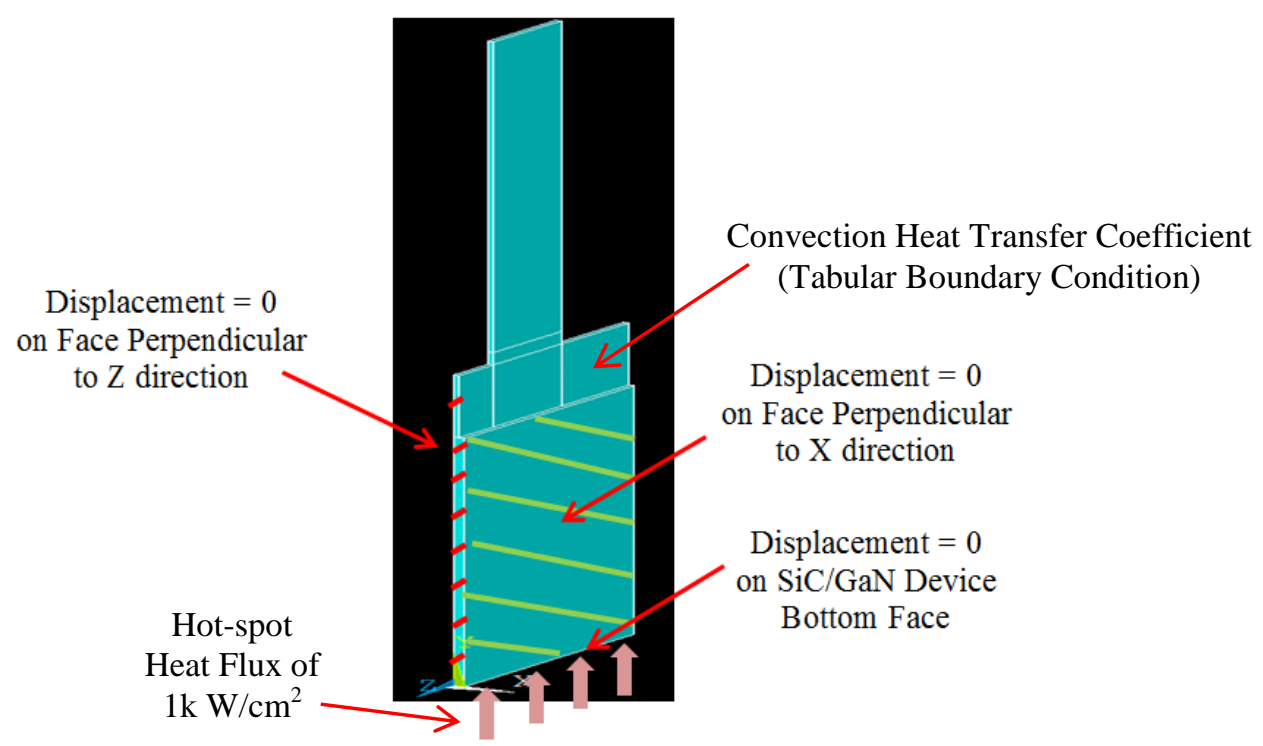

Figure 4.37: Illustration of Thermal and Mechanical Boundary Conditions 


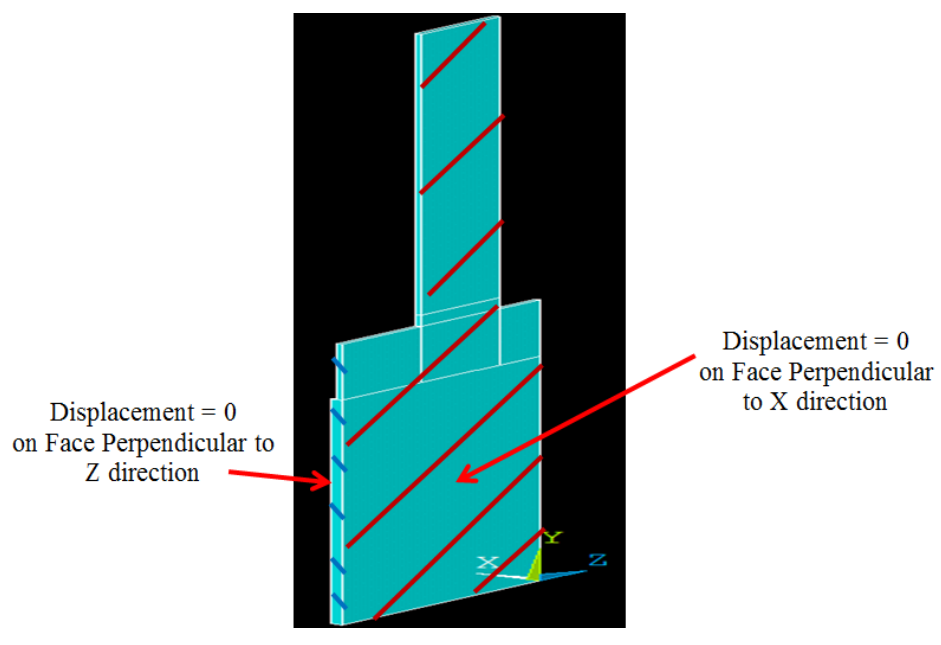

Figure 4.38: Illustration of Mechanical Boundary Conditions 


\section{Chapter 5: Results: Thermo-mechanical Structural Analysis}

In this chapter, thermo-mechanical stress analysis results will be presented. Firstly, structural modeling results of Mini-contact based thermo-electric cooling will be covered for the seven different cases of Mini-contact integration. The same will be followed by stress analysis across the Micro-fin structure.

\subsection{Integrated SiC Mini-contact based Thermoelectric Cooling}

\subsubsection{Temperature Contour Plot}

The temperature distribution across the package assembly is shown in figure 5.1. The figure illustrates the heat pumping action of the thermo-electric cooler where heat is pumped from bottom side (gets cold by rejection of heat) to top side (gets hot by absorption of heat).

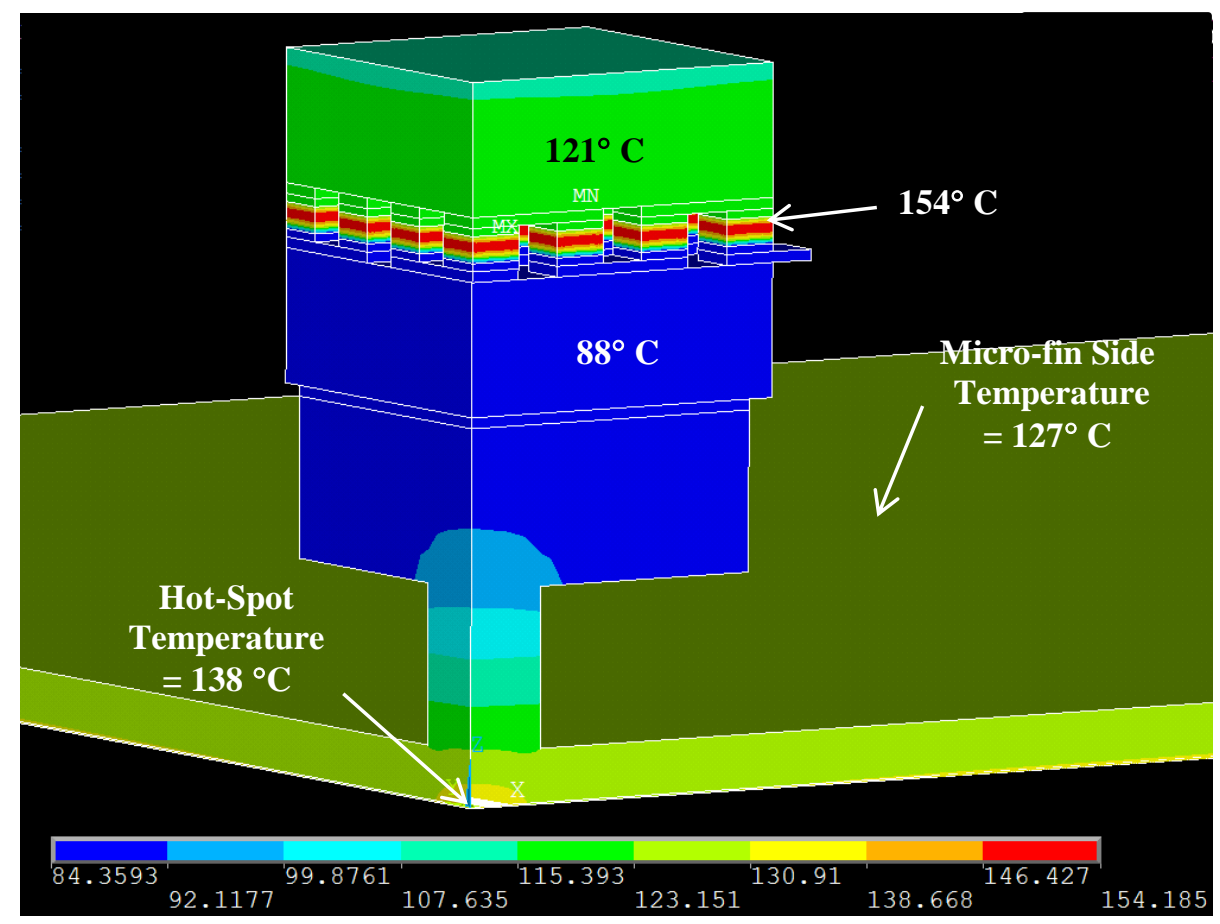

Figure 5.1: Temperature Contour Plot (Integrated SiC Mini-contact) 
A maximum hot-spot temperature of $138^{\circ} \mathrm{C}\left(137.77^{\circ} \mathrm{C}\right)$ was computed for an integrated SiC Mini-contact model. The temperature at the micro-fin side was found to be around $127^{\circ} \mathrm{C}\left(127.47^{\circ} \mathrm{C}\right)$ away from hot-spot region.

\subsubsection{Maximum Deformation Plot}

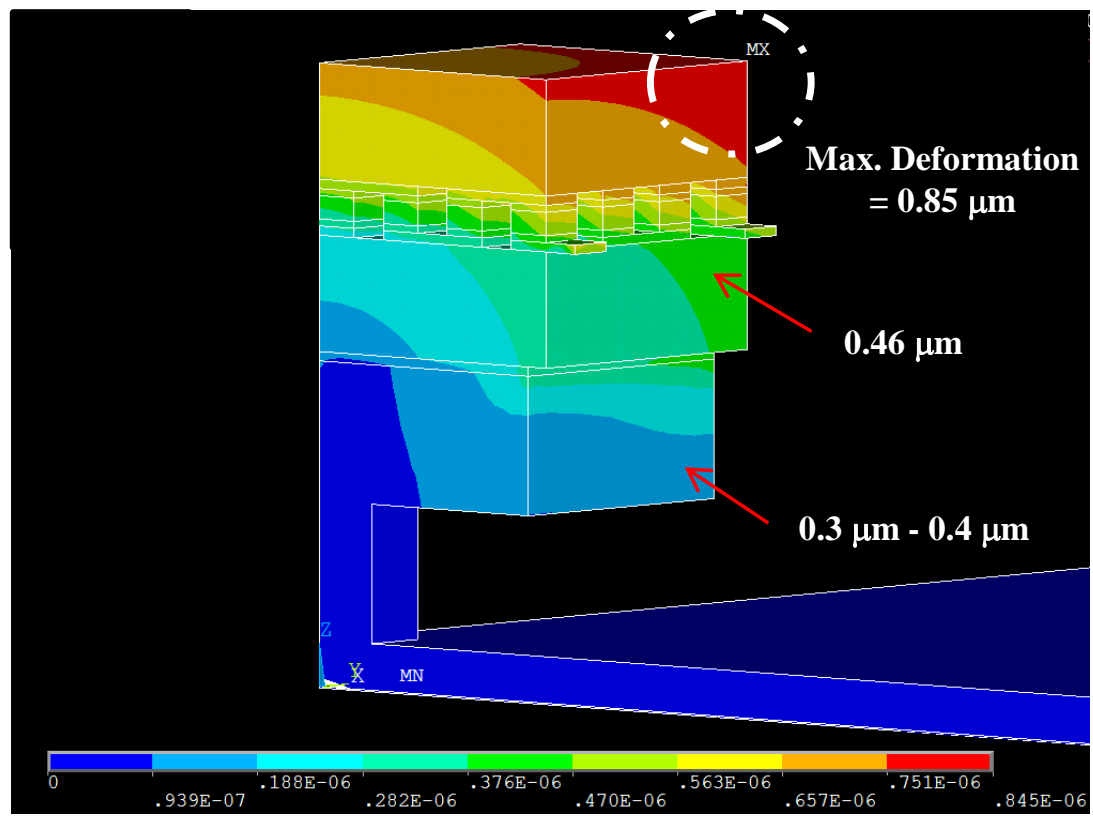

Figure 5.2: Maximum Displacement Plot (Integrated SiC Mini-contact)

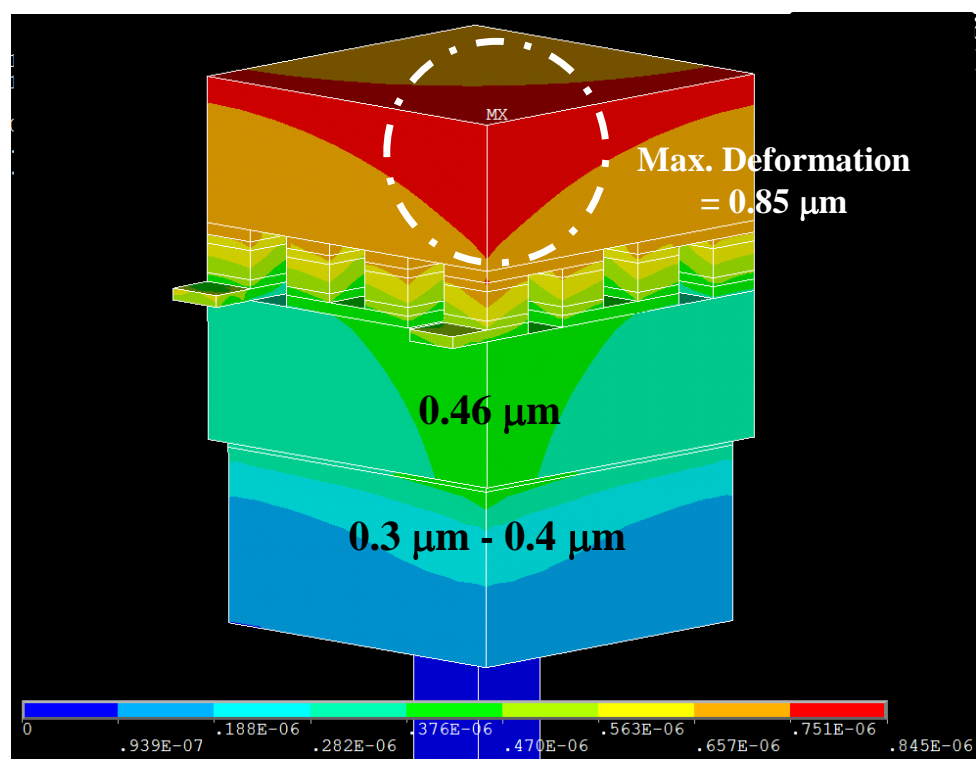

Figure 5.3: Location of Maximum Displacement (Integrated SiC Mini-contact) 
As shown in the figures 5.2 and 5.3 above, a maximum displacement of 0.85 $\mu \mathrm{m}$ was observed at the top edge of thermo-electric cooler header. This displacement is the resultant sum of expansion in all three directions. This is based on the rationale that if we supply heat to a body (figure 5.4) which is constrained only at the bottommost surface, the structure will thermally expand in all other directions.
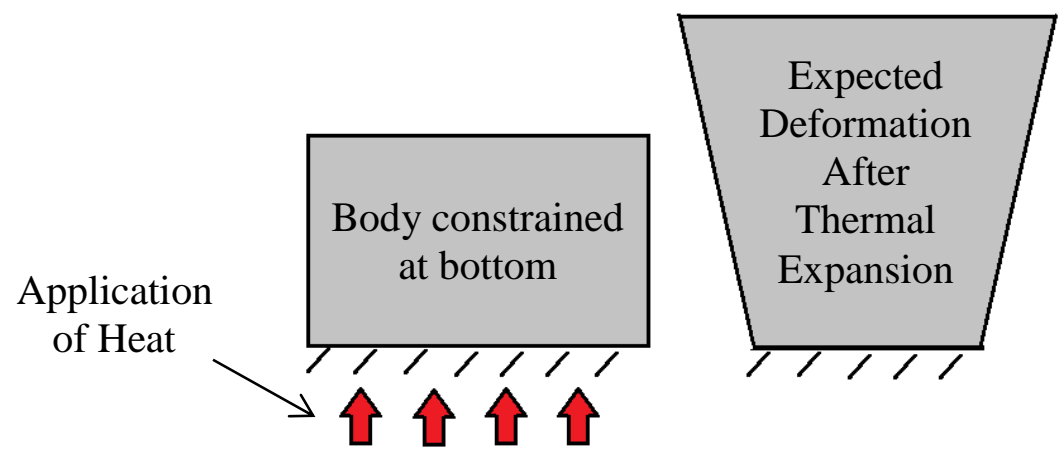

Figure 5.4: Illustration of Thermal Expansion Effect

\subsubsection{Von-Mises Plastic Strain Plot}

Power electronics are subjected to both temperature and power cycling. Passive temperature cycles arising from changes in the ambient temperature are of long duration, while power cycles are typically of shorter duration and higher frequency. With such a loading cycle, it is expected that the electronics device will fail by cyclic thermo-mechanical fatigue at different levels inside the packaging. Solder is a ductile material and such materials are known to fail by ductile fracture by plastic deformation. Hence, on comparison of the Von-Mises plastic strain in solder with its yield point, one can decide if the solder joint will undergo plastic deformation. Based on the magnitude of plastic strain in solder, we can evaluate its reliability by input of plastic strains and solder temperature to the failure model (Coffin Manson or Engelmaier's Failure Model). [22, 23] 
For an integrated SiC Mini-contact, the integration levels are Mini-contact/TE Cooler interface and interconnection of Bismuth Telluride $\left(\mathrm{Bi}_{2} \mathrm{Te}_{3}\right)$ elements with metallized Copper deposited on AlN headers. The plastic strains at these levels are presented below.

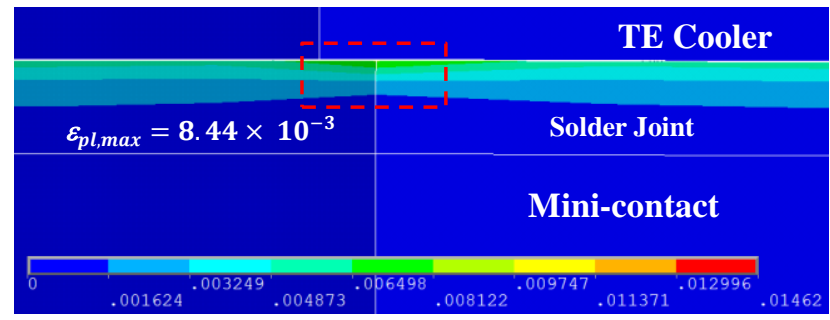

(a)

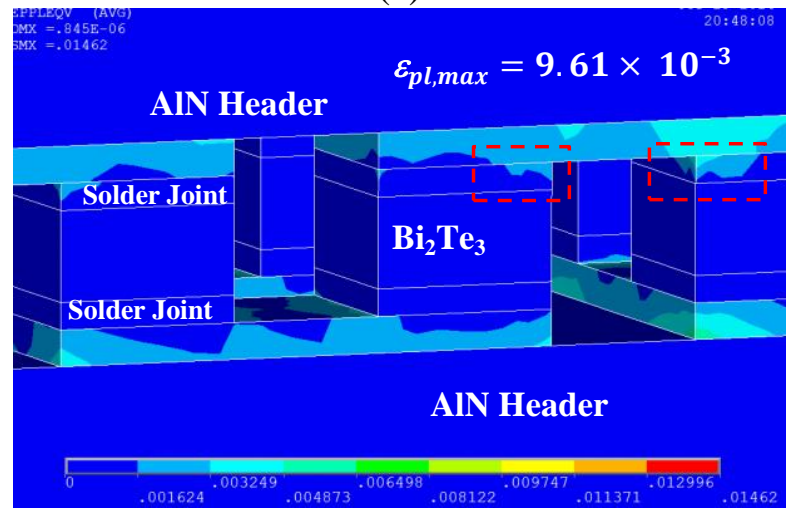

(b)

Figure 5.5: Von-Mises Plastic Strain Plot - (a) Solder Joint at Mini-contact/TE Cooler Header Interface \& (b) Solder Joint at TE elements/Copper Straps Interface

Since the solder material used inside actual thermo-electric device (HV 37 Laird Thin Film device) was unknown, it was assumed that the solder joint used for interconnection of thermo-electric element with Copper on headers is a SAC305. This assumption was based on two facts, firstly, SAC305 is known to be a good material for temperatures in range of $100{ }^{\circ} \mathrm{C}-150{ }^{\circ} \mathrm{C}$, and secondly, its elastic plastic properties are available in literature, which will assist in estimation of plastic strain and can be input to the available failure model (Engelmaier's model) to predict it's 
Mean Time to Failure (MTTF). From figure 5.5 above, the plastic strain in the solder joint at the cooler/Mini-contact level is of magnitude $8.44 \times 10^{-3}$ and inside the thermo-electric cooler; the plastic strain is of magnitude $9.61 \times 10^{-3}$.

The power electronics device undergoes power cycling, as a result of which we the power electronics undergoes repeated on/off cycles. Based on the data available from DARPA proposal for ICECool Fundamentals [50], it was assumed that the life time of one cycle is 10 hours. It is observed in general that a power electronics switch will rise to its peak temperature in a short span of time (in a few minutes), and thus, it was assumed that the device takes about 2 minutes to rise to its peak temperature and 2 minutes to drop off to room temperature of $25^{\circ} \mathrm{C}$. Dwell time refers to the amount of time the power electronics device will remain at its peak temperature. Based on data above, the dwell time was determined to be 596 minutes. Additionally, two more power cycles were considered. One power cycle of 1 hour with 20 minutes dwell at high and another one with three minutes on and three minutes off with 1 minute dwell on. In order to predict the reliability of a solder joint, we'll need three inputs, which are the dwell time, average temperature of the solder (mean of peak and room temperature values) and the plastic strain of the solder, which was determined from the FE simulations.

As mentioned earlier, Engelmaier's model is an update to Coffin Manson's model and incorporates parameters like cyclic frequency (modified to dwell time), stress relaxation effects and solder (and substrate) temperature. Thus, it gives a more accurate prediction to solder joint reliability life as compared to the original Coffin Manson Model. For reporting purposes, reliability prediction using input to 
Engelmaier's model will be provided. The output of Engelmaier's model is characteristic life of the solder, which is a $50 \%$ of product failure life time.

The Mean Time to Failure (MTTF) was calculated based on the Weibull distribution function as follows.

$$
M T T F=\gamma+\eta *\left\lceil\left(\frac{1}{\beta}+1\right)\right.
$$

where,

$\eta$ is scale parameter or characteristic life

$\beta$ is shape parameter (or slope)

$\gamma$ is location parameter (or failure free life)

A failure free life means a $\gamma=0 . \eta=N_{f}(50 \%)$. The value of $\beta$ has a marked effect on the failure rate. The three life stages of a bathtub curve can be modeled with the help of a Weibull distribution and varying values of $\beta$. The failure rate in general increases with time and a $\beta=3$ represents a normal distribution of probability density function on the Weibull curve $[16,17]$. Thus, one can differentiate between $\mathrm{N}_{\mathrm{f}}(50 \%)$ and MTTF reliability prediction values using the above equation.

Tables 5.1 and 5.2 give the reliability prediction (Mean Time to Failure, MTTF) of solder joint interconnecting Mini-contact with thermo-electric cooler and thermo-electric elements with Copper on headers.

The $\mathrm{N}_{\mathrm{f}}(50 \%)$ for Mini-contact - TE Cooler integration for dwells of 596 minutes, 20 minutes and 1 minute were found out to be 5,024 cycles, 10234 cycles and 47024 cycles. The $\mathrm{N}_{\mathrm{f}}(63.2 \%)$ for integration at this level were found out to be 5,677 cycles, 11562 cycles and 53128 cycles. The MTTF values are shown below. 
Table 5.1: Reliability Predictions

Solder Joint - Mini-contact/TE Cooler Header Interface

\begin{tabular}{|c|c|}
\hline Maximum Plastic Strain & $8.44 \mathrm{e}-3$ \\
\hline Maximum Temperature & $85.69^{\circ} \mathrm{C}$ \\
\hline Minimum Temperature & $25^{\circ} \mathrm{C}$ \\
\hline Average Temperature & $55.34^{\circ} \mathrm{C}$ \\
\hline Node Number & 48980 \\
\hline MTTF (596 mins. dwell) & $\mathbf{3 , 8 6 7}$ cycles \\
\hline MTTF (20 mins. dwell) & $\mathbf{7 , 8 7 6}$ cycles \\
\hline MTTF (1 min. dwell) & $\mathbf{3 6 1 , 1 9 1}$ cycles \\
\hline
\end{tabular}

The next integration level considered was interconnection of thermo-electric elements with Copper on AlN header. The $\mathrm{N}_{\mathrm{f}}(50 \%)$ for three cases of dwell times (596 mins., 20 mins. and 1 min.) mentioned before were determined out to be 2,649 cycles, 4,998 cycles and 19,234 cycles. The $\mathrm{N}_{\mathrm{f}}(63.2 \%)$ was found out to be 2,994 cycles, 5,646 cycles and 21,731 cycles.

Table 5.2: Reliability Prediction (MTTF) Solder Joint - Copper Straps/Thermoelectric Elements Interface

\begin{tabular}{|c|c|}
\hline Maximum Plastic Strain & $9.61 \mathrm{e}-3$ \\
\hline Maximum Temperature & $123.49^{\circ} \mathrm{C}$ \\
\hline Minimum Temperature & $25^{\circ} \mathrm{C}$ \\
\hline Average Temperature & $74.25^{\circ} \mathrm{C}$ \\
\hline Node Number & 114224 \\
\hline MTTF (596 mins. dwell) & $\mathbf{2 , 0 3 9}$ cycles \\
\hline MTTF (20 mins. dwell) & $\mathbf{3 , 8 4 7}$ cycles \\
\hline MTTF (1 min. dwell) & $\mathbf{1 4 , 8 0 3}$ cycles \\
\hline
\end{tabular}

Based on Tables 5.1 and 5.2, one can infer that the solder joints interconnecting the Mini-contact with the thermo-electric cooler will exhibit their first failure after either 3,867 cycles, 7,876 cycles or 361,191 cycles depending on dwell time. The solder joint interconnecting the Bismuth Telluride $\left(\mathrm{Bi}_{2} \mathrm{Te}_{3}\right)$ elements with metallized Copper deposited on AIN headers will exhibit their first failure either after 2,039 cycles, 3,847 cycles or 14,803 cycles depending on dwell time. The marked 
distinction in these reliability values can be attributed to difference in plastic strain and average solder temperature values.

There are two levels of solder joint interconnection inside the thermo-electric cooler, top and bottom. The solder joint above the cooler will fail first in comparison to the solder joint at bottom because it experiences higher temperature. The solder joint below the cooler is predicted to fail after a cycle period of 4566 cycles, 8029 cycles or 37,032 cycles.

\subsubsection{Critical Flaw Size - Bismuth Telluride Elements - $\mathrm{Bi}_{2} \mathrm{Te}_{3}$}

The thermo-electric cooler consists of materials like AlN (headers) and $\mathrm{Bi}_{2} \mathrm{Te}_{3}$ (thermo-electric element) that fail by brittle fracture. Linear elastic fracture mechanics can be used to study crack propagation in brittle materials. From thermomechanical stress analysis, we determined the stress concentrations in $\mathrm{Bi}_{2} \mathrm{Te}_{3}$ elements and AlN headers, which were tensile in nature, and then used fracture mechanics to predict the critical flaw size. Based on the size of flaw, we can conclude if these stress levels will be likely to lead to brittle fracture in material.

To study crack propagation in brittle materials, we need fracture toughness of the material and max stress magnitude, which can be input in the following equation to predict critical flaw size based on fracture mechanics approach [8].

$$
\begin{gathered}
K_{I C}=Y \sigma_{\max } \sqrt{\pi a_{c}} \\
a_{c}=\frac{1}{\pi}\left(\frac{K_{I C}}{\sigma Y}\right)^{2}
\end{gathered}
$$

The $\sigma_{\max }$ value is obtained through thermo-mechanical stress modeling. One of the important assumptions to be made with the fracture mechanics approach is the 
consideration if the initial crack is present inside the material or it's on the material edge. Based on stress concentrations received on the elements and headers, it was assumed that initial crack is present on the edge. Thus, the factor $\mathrm{Y}$ is assumed to be 1.2 .

Figures 5.6 - 5.8 (below) give the principal stress plots in Bismuth Telluride elements for an integrated SiC Mini-contact. As per the maximum principal stress theory, a maximum stress out of the three given stresses will be used for calculation of the critical crack size based on the theory of fracture mechanics. The maximum stresses were seen on nodes at outer edge of thermo-electric elements.

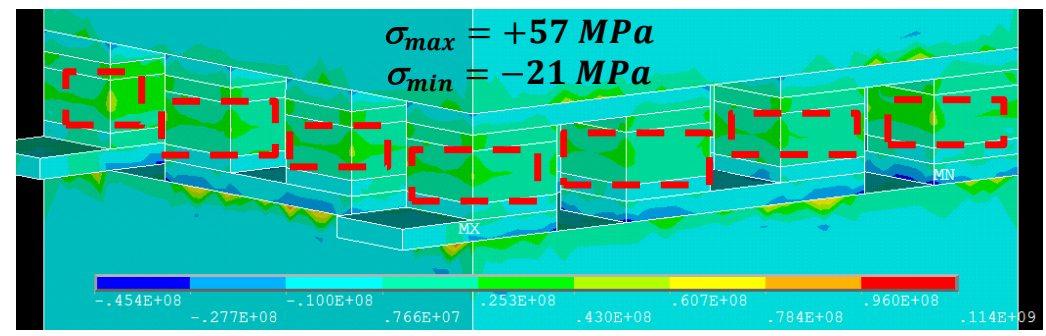

Figure 5.6: $1^{\text {st }}$ Pr. Stress Contour Plot - TE Elements

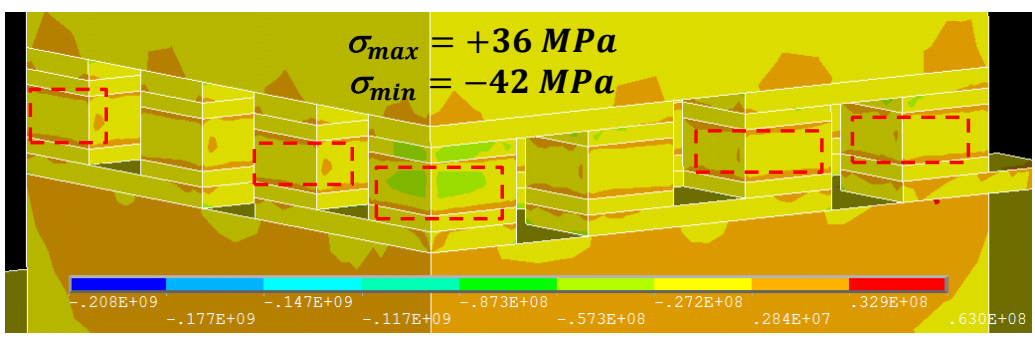

Figure 5.7: $2^{\text {nd }}$ Pr. Stress Contour Plot - TE Elements

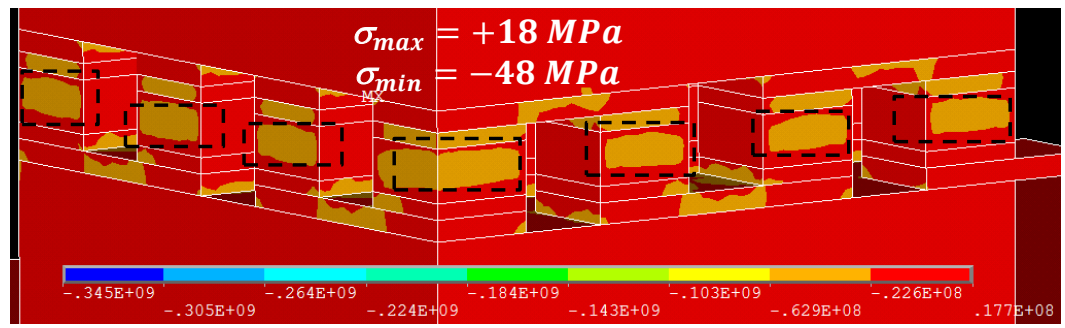

Figure 5.8: $3^{\text {rd }}$ Pr. Stress Contour Plot - TE Elements 
Table 5.3: Principal Stresses in Thermoelectric Element

\begin{tabular}{|c|c|c|c|}
\hline Parameter & $\begin{array}{c}\mathbf{1}^{\text {st }} \text { Principal } \\
\text { Stress }\end{array}$ & $\begin{array}{c}\mathbf{2}^{\text {nd }} \text { Principal } \\
\text { Stress }\end{array}$ & $\begin{array}{c}\mathbf{3}^{\text {rd }} \text { Principal } \\
\text { Stress }\end{array}$ \\
\hline Maximum Stress & $\mathbf{5 7} \mathrm{MPa}$ & $36 \mathrm{MPa}$ & $18 \mathrm{MPa}$ \\
\hline Node Number & 59562 & 80694 & 68763 \\
\hline Minimum Stress & $-21 \mathrm{MPa}$ & $-42 \mathrm{MPa}$ & $-48 \mathrm{MPa}$ \\
\hline Node Number & 88249 & 88578 & 88565 \\
\hline
\end{tabular}

The fracture toughness of $\mathrm{Bi}_{2} \mathrm{Te}_{3}$ isn't available in literature. However a survey of typical piezoelectric ceramics indicated fracture toughness in the range of $1 M P a * m^{1 / 2}-2 M P a * m^{1 / 2}$. For a stress magnitude of $57 \mathrm{MPa}, \mathrm{Y}=1.2$, the critical flaw size was calculated in between $68 \mu \mathrm{m}-272 \mu \mathrm{m}$. Considering the size of elements $(15 \mu \mathrm{m})$, this flaw size is relatively large and thus, stresses are deemed safe.

\subsubsection{Critical Flaw Size - Aluminum Nitride Headers}

The principal stress plot in AlN headers is shown below in figures 5.9 - 5.11.

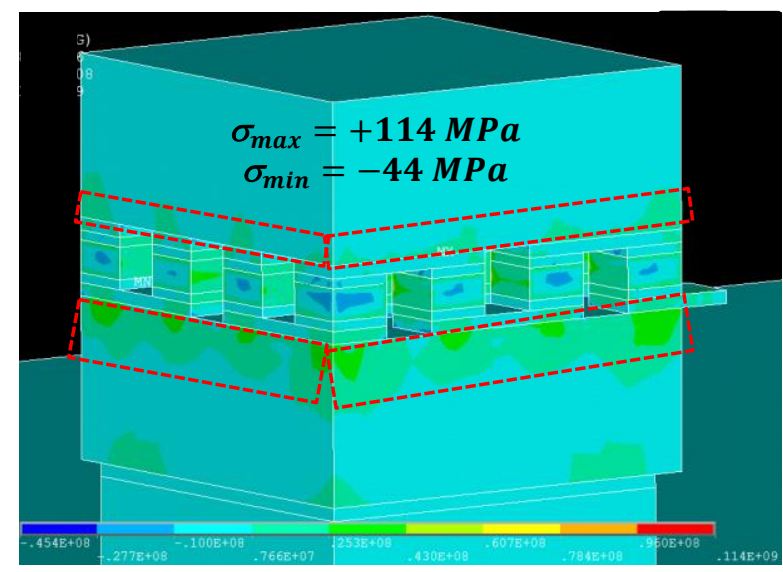

Figure 5.9: $1^{\text {st }}$ Pr. Stress Plot - TE Cooler Headers 


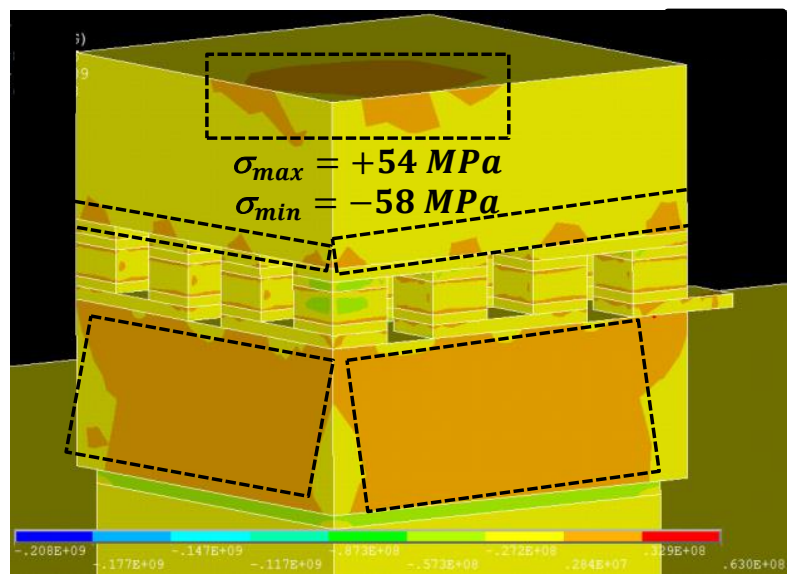

Figure 5.10: $2^{\text {nd }}$ Pr. Stress Plot - TE Cooler Headers

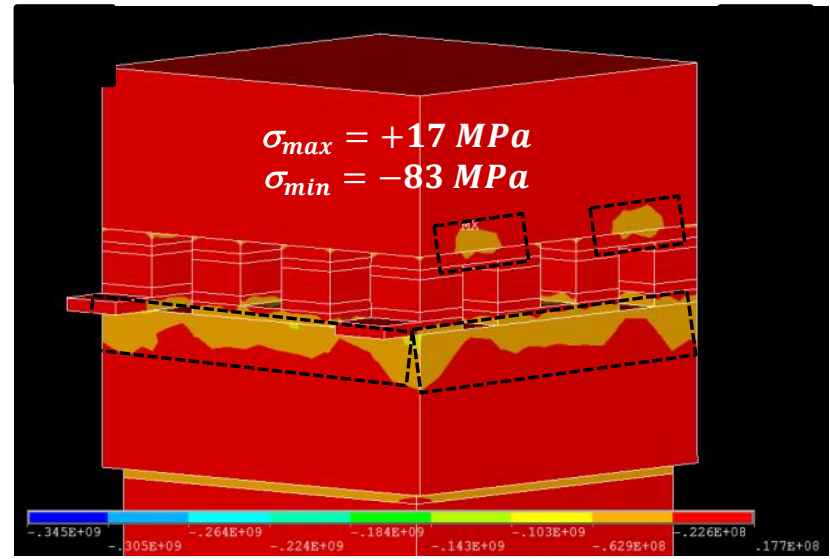

Figure 5.11: $3^{\text {rd }}$ Pr. Stress Plot - TE Cooler Headers

Table 5.4: Principal Stresses in AlN Headers

\begin{tabular}{|c|c|c|c|}
\hline Parameter & $\begin{array}{c}\mathbf{1}^{\text {st }} \text { Principal } \\
\text { Stress }\end{array}$ & $\begin{array}{c}\mathbf{2}^{\text {nd }} \text { Principal } \\
\text { Stress }\end{array}$ & $\begin{array}{c}\mathbf{3}^{\text {rd }} \text { Principal } \\
\text { Stress }\end{array}$ \\
\hline $\begin{array}{c}\text { Maximum } \\
\text { Stress }\end{array}$ & $114 \mathrm{MPa}$ & $54 \mathrm{MPa}$ & $17 \mathrm{MPa}$ \\
\hline Node Number & 108470 & 108470 & 126366 \\
\hline $\begin{array}{c}\text { Minimum } \\
\text { Stress }\end{array}$ & $-44 \mathrm{MPa}$ & $-58 \mathrm{MPa}$ & $-83 \mathrm{MPa}$ \\
\hline Node Number & 107526 & 107526 & 108470 \\
\hline
\end{tabular}

A maximum principal stress of $114 \mathrm{MPa}$ was calculated through Finite

Element simulations. A fracture toughness value of 2.6 $\mathrm{MPam}^{1 / 2}$ [51] was used in the critical flaw size calculation equation. $\mathrm{Y}=1.2$ was used. Based on these input 
parameters, a critical flaw size of $115 \mu \mathrm{m}$ was calculated, which is a relatively large flaw size. Hence, the stresses are deemed under safe limit.

\subsubsection{Critical Flaw Size - GaN on SiC}

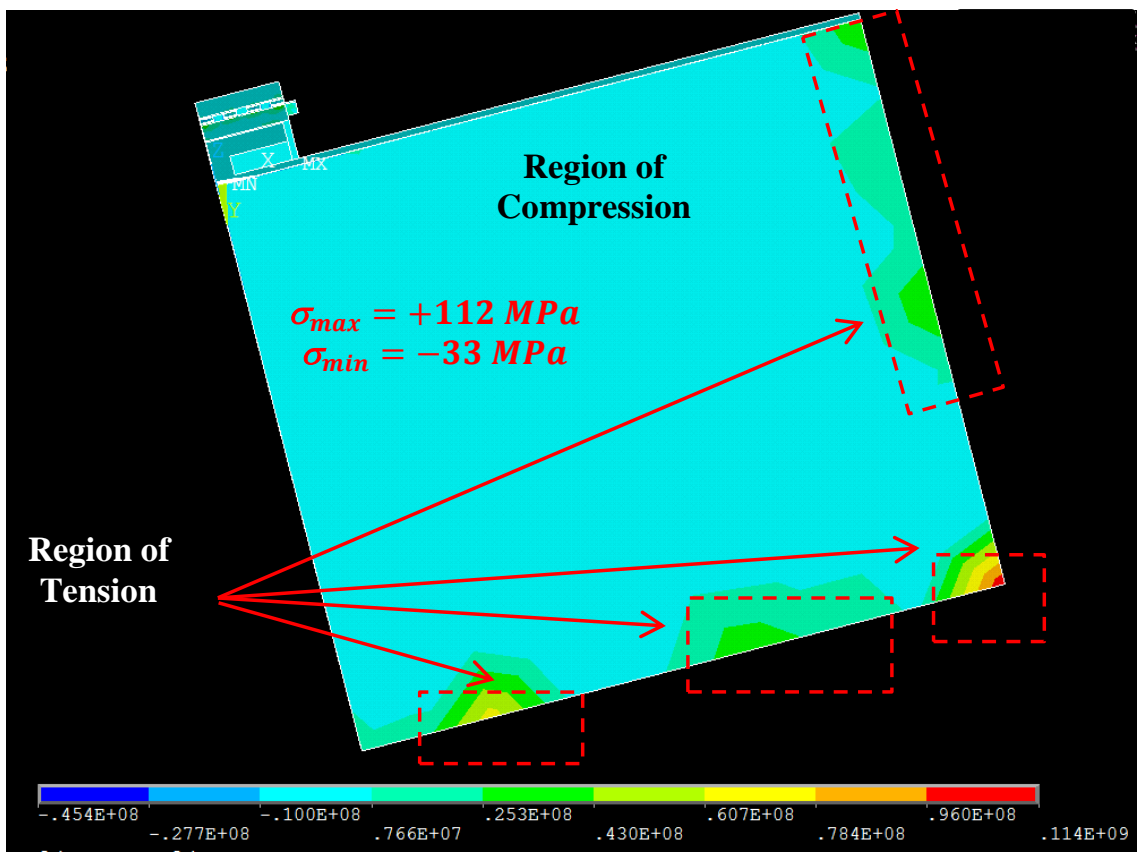

Figure 5.12: $1^{\text {st }}$ Pr. Stress Plot - GaN on SiC

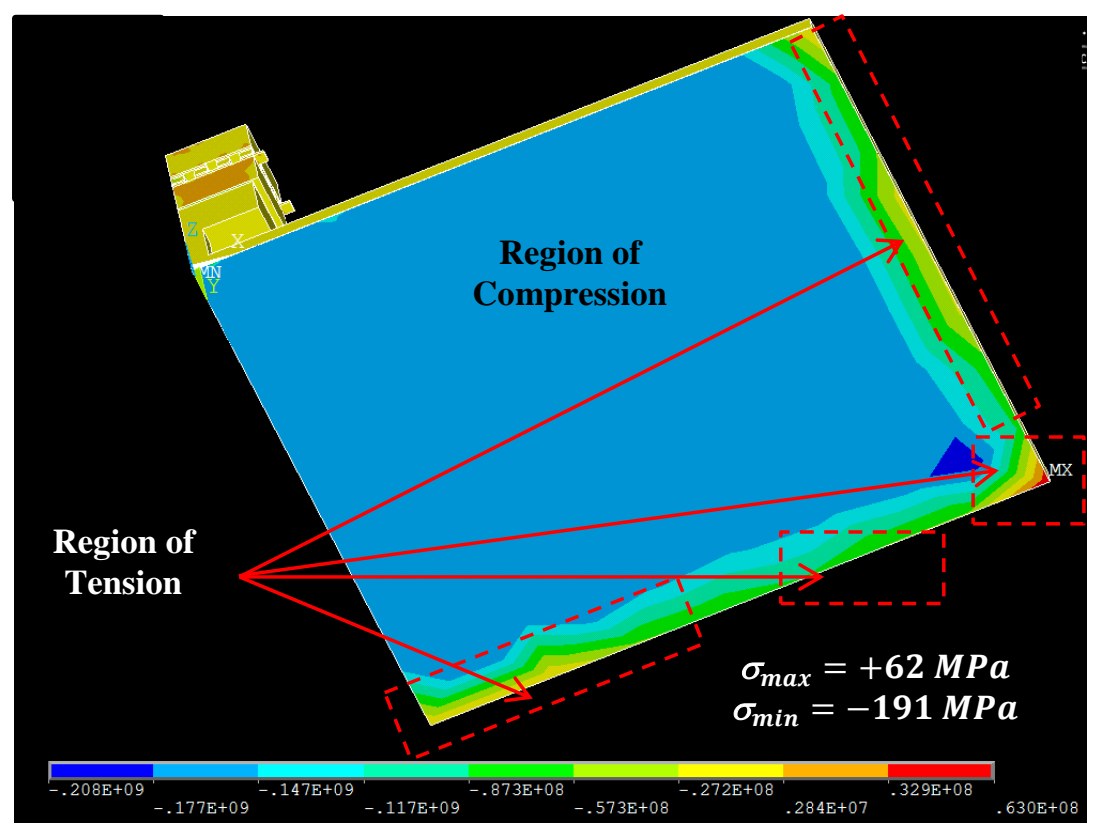

Figure 5.13: $2^{\text {nd }}$ Pr. Stress Plot - GaN on SiC 


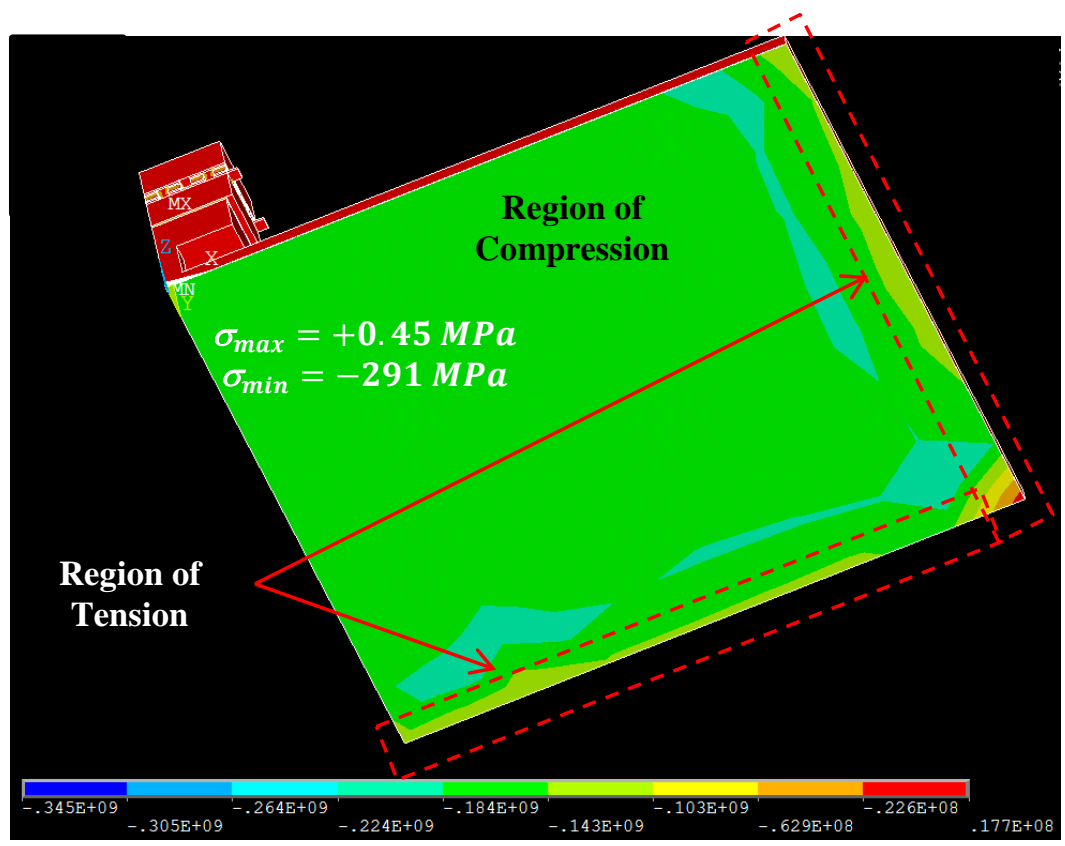

Figure 5.14: $3^{\text {rd }}$ Pr. Stress Plot - GaN on SiC Device

Table 5.5 Principal Stresses - GaN on $\mathrm{SiC}$

\begin{tabular}{|c|c|c|c|}
\hline Parameter & $\begin{array}{c}\mathbf{1}^{\text {st }} \text { Principal } \\
\text { Stress }\end{array}$ & $\begin{array}{c}\mathbf{2}^{\text {nd }} \text { Principal } \\
\text { Stress }\end{array}$ & $\begin{array}{c}\mathbf{3}^{\text {rd }} \text { Principal } \\
\text { Stress }\end{array}$ \\
\hline Maximum & $\mathbf{1 1 2} \mathbf{M P a}$ & $62 \mathrm{MPa}$ & $0.45 \mathrm{MPa}$ \\
\hline Node Number & $\mathbf{1 6 9 1 7 5}$ & 169175 & 169175 \\
\hline Minimum & $-33 \mathrm{MPa}$ & $-191 \mathrm{MPa}$ & $-291 \mathrm{MPa}$ \\
\hline Node Number & 2919 & 169517 & $\mathbf{2 9 1 9}$ \\
\hline
\end{tabular}

From the above plots (figure 5.12 - 5.14), a maximum stress of $112 \mathrm{MPa}$ was seen at the edge of the $\mathrm{GaN}(3 \mu \mathrm{m})$ on SiC device. To calculate the critical flaw size, a fracture toughness value of $1.1 \mathrm{MPam}^{1 / 2}$ [52], $\mathrm{Y}=1.2$ and stress of $112 \mathrm{MPa}$ was input to the fracture mechanics equation. A critical flaw size of $21 \mu \mathrm{m}$ was calculated. On the other hand, there are compressive stresses in the GaN device, primarily at the center of the device, magnitude of -291 MPa. Both tensile and compressive stresses can adversely affect the device performance by piezoelectric effects. 


\subsection{Discrete Mini-contact: Copper - T-shaped Model}

5.2.1 Nodal Temperature Plot

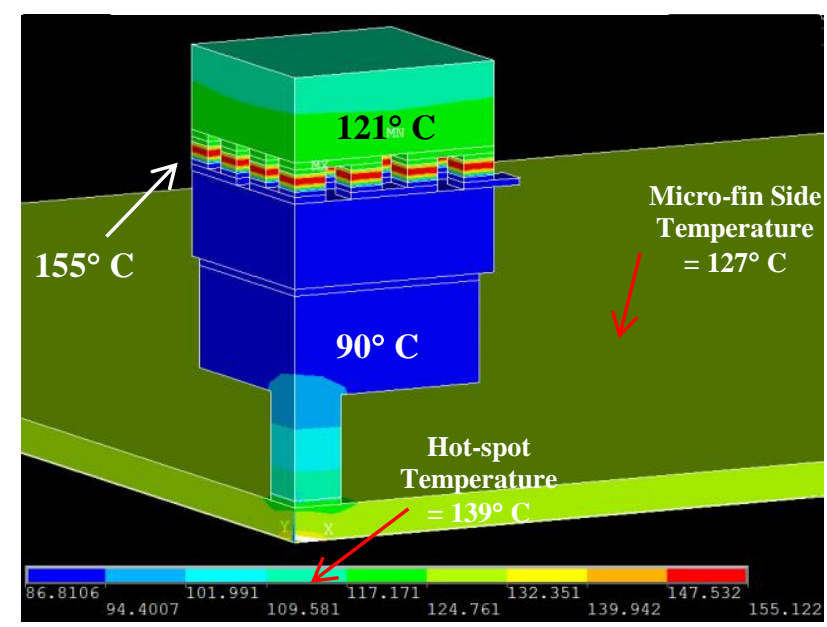

Figure 5.15: Temperature Contour Plot

As it can be inferred from the figure above, a maximum hot-spot temperature

of $139{ }^{\circ} \mathrm{C}$ was observed for the $\mathrm{T}$-shaped mini-contact. The temperature on the microfin side was calculated by the FE software as $127^{\circ} \mathrm{C}$.

\subsubsection{Displacement Contour Plot}

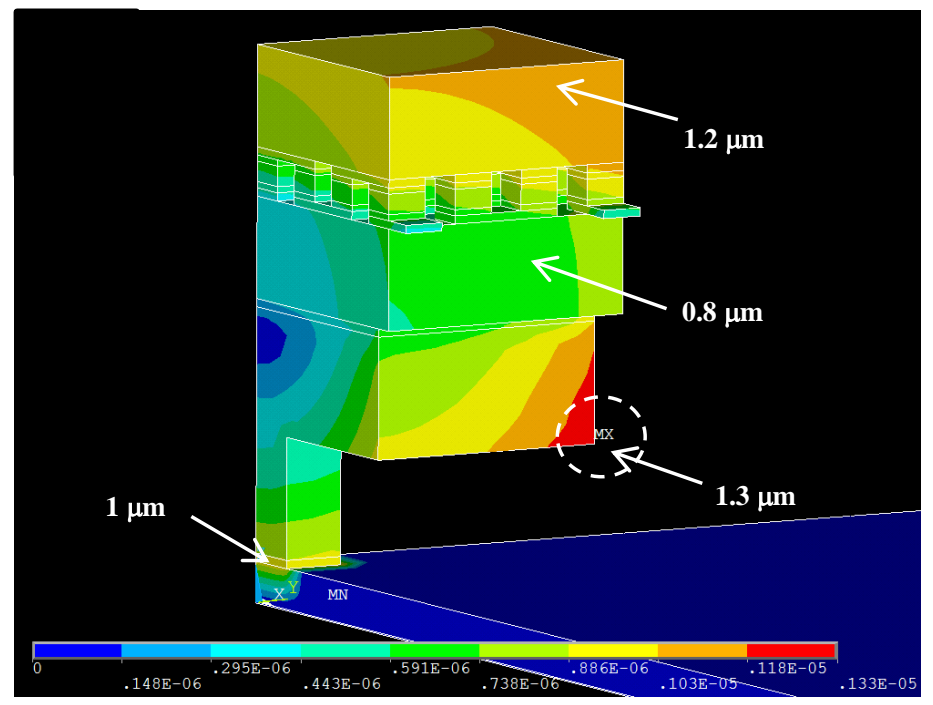

Figure 5.16: Displacement Contour Plot 
Figure 5.16 gives an overview of the maximum displacement observed in a Tshaped Copper Mini-contact assembly. A maximum displacement of $1.3 \mu \mathrm{m}$ is seen at the tip of Copper Mini-contact, which is followed by displacement in thermoelectric cooler header top edge and chip level solder joint interconnection. Copper and SAC305 solder both have relatively high C.T.E. and thus, one can expect when a discrete Copper Mini-contact/Cooler assembly is heated, these materials will experience comparatively higher deformation.

\subsubsection{Von-Mises Plastic Strain Plot}

Figures 5.17 and 5.18 give the distribution of Von-Mises plastic strain across two levels of integration. Figure 5.17 corresponds to the plastic strain in solder at Chip/Mini-contact level integration. The strain at cooler - Mini-contact integration is shown in figure 5.18 .

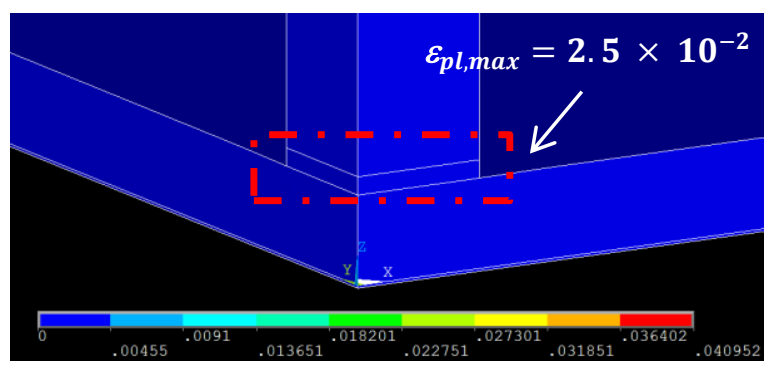

Figure 5.17: Plastic Strain Plot - Solder Joint at Chip/Mini-contact Interface 


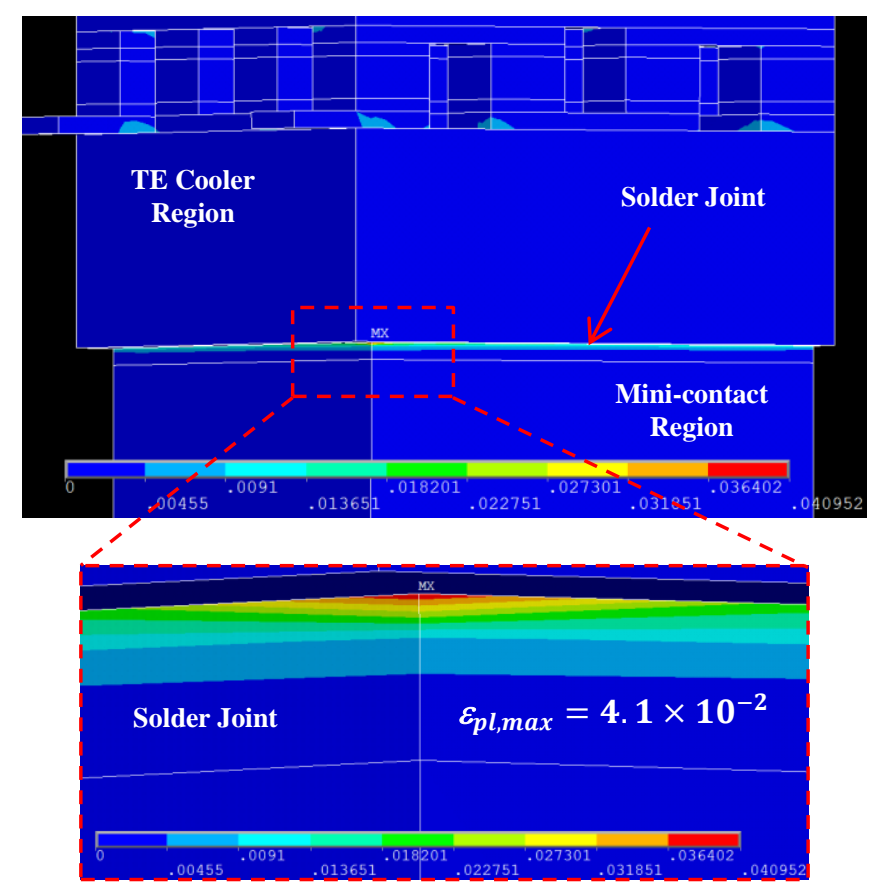

Figure 5.18: Pl. Strain - Solder Joint: TE Cooler/Mini-contact

At chip level integration, a plastic strain of $2.5 \times 10^{-2}$ is observed. The solder joint interconnecting thermo-electric cooler with Mini-contact undergoes a plastic strain of $4.1 \times 10^{-2}$. The corresponding MTTF values are given in tables 5.6 and 5.7.

$\mathrm{N}_{\mathrm{f}}(50 \%)$ for chip level interconnection: 51, 065 cycles, 120,120 cycles and 739,591 cycles. $\mathrm{N}_{\mathrm{f}}(63.2 \%)$ for this level: 56,670 cycles, 135,711 cycles and 835,589 cycles. $\mathrm{N}_{\mathrm{f}}(50 \%)$ for Mini-contact - Cooler integration: 135 cycles, 208 cycles and 525 cycles. $\mathrm{N}_{\mathrm{f}}(63.2 \%)$ for this level: 153 cycles, 235 cycles and 593 cycles.

Table 5.6: Design for Reliability (MTTF) - Chip Level Integration SiC - Solder Joint - Mini-contact

\begin{tabular}{|c|c|}
\hline Maximum Plastic Strain & $2.52 \mathrm{e}-3$ \\
\hline Maximum Temperature & $121.48^{\circ} \mathrm{C}$ \\
\hline Minimum Temperature & $25^{\circ} \mathrm{C}$ \\
\hline Average Temperature & $73.24^{\circ} \mathrm{C}$ \\
\hline Node Number & 24312 \\
\hline MTTF (596 mins.) & $\mathbf{3 9 , 3 0 0 ~ c y c l e s ~}$ \\
\hline MTTF (20 mins.) & $\mathbf{9 2 , 4 4 7}$ cycles \\
\hline MTTF (1 min.) & $\mathbf{5 6 9 , 2 0 5}$ cycles \\
\hline
\end{tabular}


Table 5.7: Design for Reliability (MTTF) - Cooler/Contact Level Integration Mini-contact - Solder Joint - Thermoelectric Cooler

\begin{tabular}{|c|c|}
\hline Maximum Plastic Strain & $4.09 \mathrm{e}-2$ \\
\hline Maximum Temperature & $88.21^{\circ} \mathrm{C}$ \\
\hline Minimum Temperature & $25^{\circ} \mathrm{C}$ \\
\hline Average Temperature & $56.61^{\circ} \mathrm{C}$ \\
\hline Node Number & 55974 \\
\hline MTTF (596 mins.) & $\mathbf{1 0 4}$ cycles \\
\hline MTTF (20 mins.) & $\mathbf{1 6 0}$ cycles \\
\hline MTTF (1 min.) & $\mathbf{4 0 4}$ cycles \\
\hline
\end{tabular}

As per the tables above, we can see that with Copper as a Mini-contact, the failure site shifts to the interconnection level between Copper Mini-contact and TE Cooler. The solder at chip level is predicted to last for either 39,300 cycles, 92,447 cycles or 569,205 cycles depending on the dwell time. At contact/cooler level, the MTTF value was found out to be 104 cycles, 160 cycles and 404 cycles. The failure inside the thermo-electric cooler is shown below.

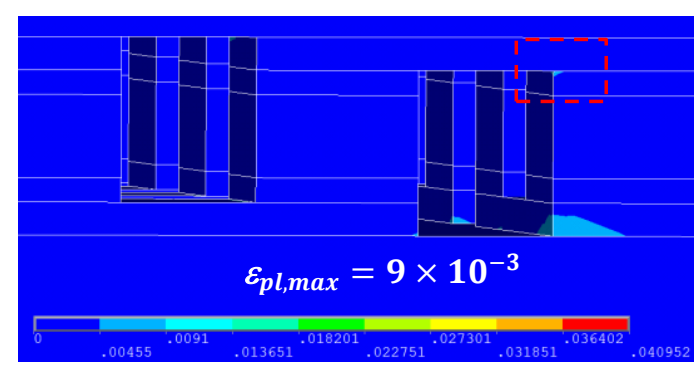

Figure 5.19: Pl. Strain - Solder Joint in TE Cooler

As mentioned earlier, there are two levels of solder joint interconnection, one above the thermo-electric element and the other joint, below the thermo-electric element. The one above thermo-electric element has higher temperature and plastic strain and thus, fails first. A plastic strain of $9 \times 10^{-3}$ was calculated for this case. The MTTF of solder joint inside thermo-electric cooler is shown below in Table 5.7. The $\mathrm{N}_{\mathrm{f}}(50 \%)$ values were determined to be 3,087 cycles, 4,538 cycles and 17,948 cycles 
for dwells of 596 mins., 20 mins. and $1 \mathrm{~min}$. The $\mathrm{N}_{\mathrm{f}}(63.2 \%)$ values were found out to be 3,487 cycles, 6,661 cycles and 26,347 cycles respectively.

Table 5.8: Design for Reliability (MTTF) - Thermo-electric Cooler Solder joint interconnecting Thermo-electric Elements with Copper on Headers

\begin{tabular}{|c|c|}
\hline Maximum Plastic Strain & $9.03 e-3$ \\
\hline Maximum Temperature & $121.68^{\circ} \mathrm{C}$ \\
\hline Minimum Temperature & $25^{\circ} \mathrm{C}$ \\
\hline Average Temperature & $73.34^{\circ} \mathrm{C}$ \\
\hline Node Number & 116095 \\
\hline MTTF (596 mins.) & 2,376 cycles \\
\hline MTTF (20 mins.) & 4,538 cycles \\
\hline MTTF (1 min.) & 17,948 cycles \\
\hline
\end{tabular}

As shown in Table 5.7, a MTTF of 2,376 cycles was calculated based on Engelmaier's failure model for a plastic strain of $9.03 \times 10^{-3}$. Thus, one can infer that the reliability of thermo-electric cooler is a function of the strength of solder joint. Next section gives details on principal stresses.

\subsubsection{Critical Flaw Size - Bismuth Telluride Elements}

The stress concentrations in thermo-electric elements and the corresponding critical flaw size calculations will be presented in this section. The first, second and third principal stress plots are shown below in figures 5.20 - 5.22.

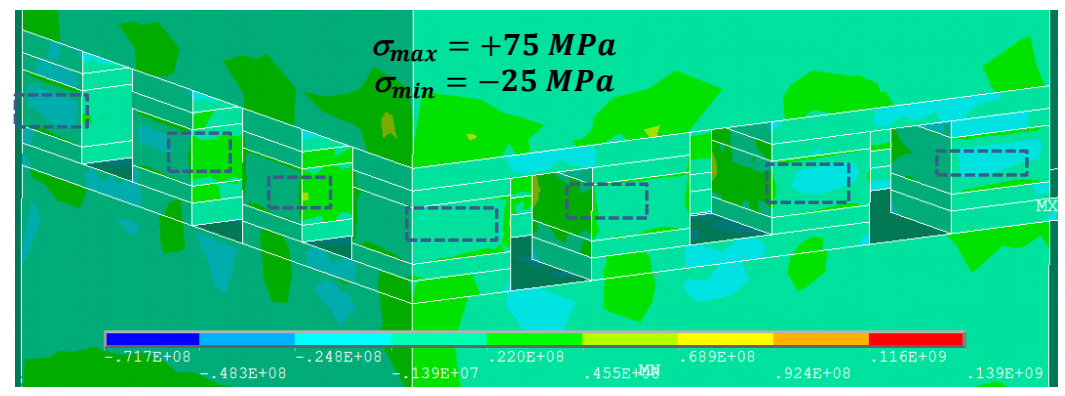

Figure 5.20: $1^{\text {st }}$ Principal Stress Plot - TE Elements 


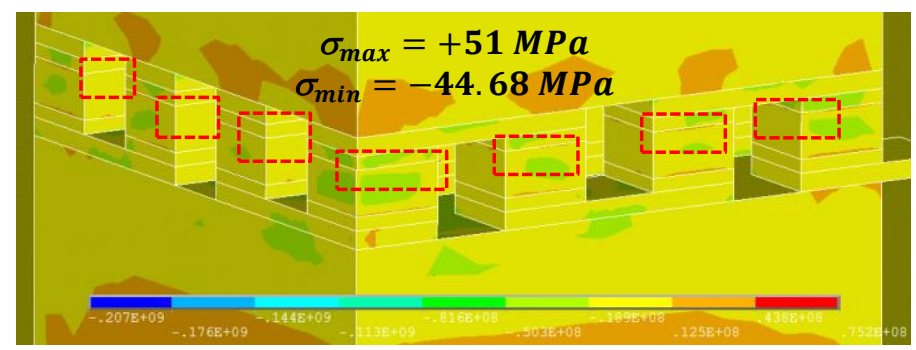

Figure 5.21: $2^{\text {nd }}$ Principal Stress Plot - TE Elements

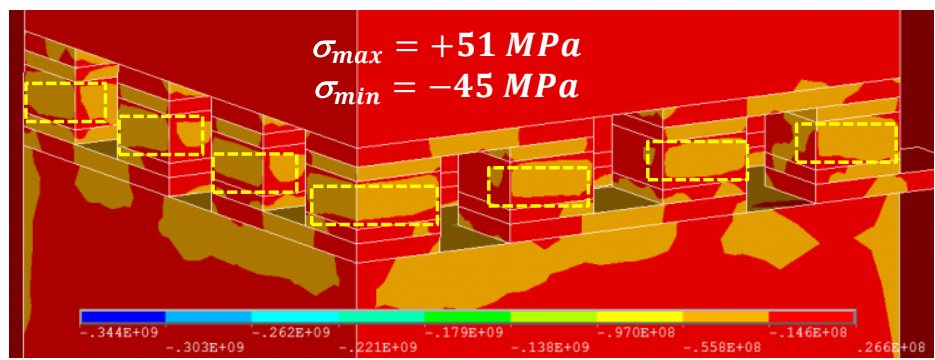

Figure 5.22: $3^{\text {rd }}$ Principal Stress Plot - TE Elements

Table 5.9: Principal Stresses - Thermoelectric Elements

\begin{tabular}{|c|c|c|c|}
\hline Parameter & $\begin{array}{c}\mathbf{1}^{\text {st }} \text { Principal } \\
\text { Stress }\end{array}$ & $\begin{array}{c}\mathbf{2}^{\text {nd }} \text { Principal } \\
\text { Stress }\end{array}$ & $\begin{array}{c}\mathbf{3}^{\text {rd }} \text { Principal } \\
\text { Stress }\end{array}$ \\
\hline Maximum & $\mathbf{7 5} \mathrm{MPa}$ & $51 \mathrm{MPa}$ & $26 \mathrm{MPa}$ \\
\hline Node Number & 95044 & 87532 & 94670 \\
\hline Minimum & $-25 \mathrm{MPa}$ & $-45 \mathrm{MPa}$ & $-56 \mathrm{MPa}$ \\
\hline Node Number & 87532 & 95369 & 87532 \\
\hline
\end{tabular}

A maximum principal stress of $75 \mathrm{MPa}$ is seen in thermo-electric elements.

The same can be input to calculate critical flaw size based on equation from fracture mechanics as presented in for integrated SiC Mini-contact model. Assuming the fracture toughness for $\mathrm{Bi}_{2} \mathrm{Te}_{3}$ to be in between $1 \mathrm{MPam}^{1 / 2}-2 \mathrm{MPam}^{1 / 2}, \mathrm{Y}=1.2$, the critical flaw size can be calculated to be in between $38 \mu \mathrm{m}-153 \mu \mathrm{m}$. For TE element size of $15 \mu \mathrm{m}$, the flaw size calculated is relatively large. 
5.2.5 Critical Flaw Size - AlN Headers

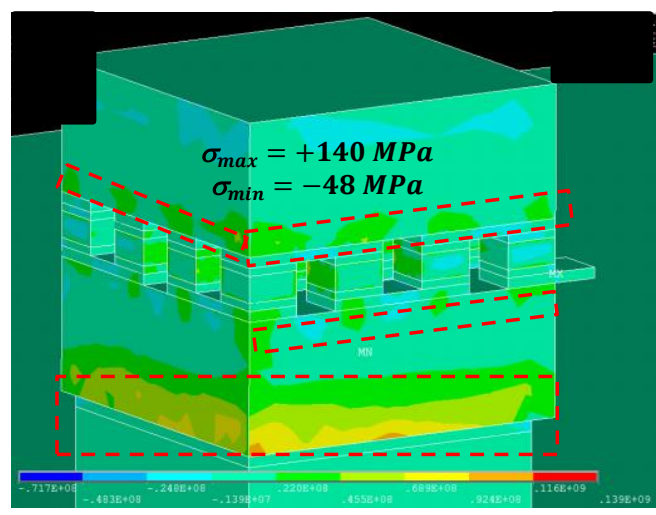

Figure 5.23: $1^{\text {st }}$ Principal Stress Plot - TE Cooler Headers

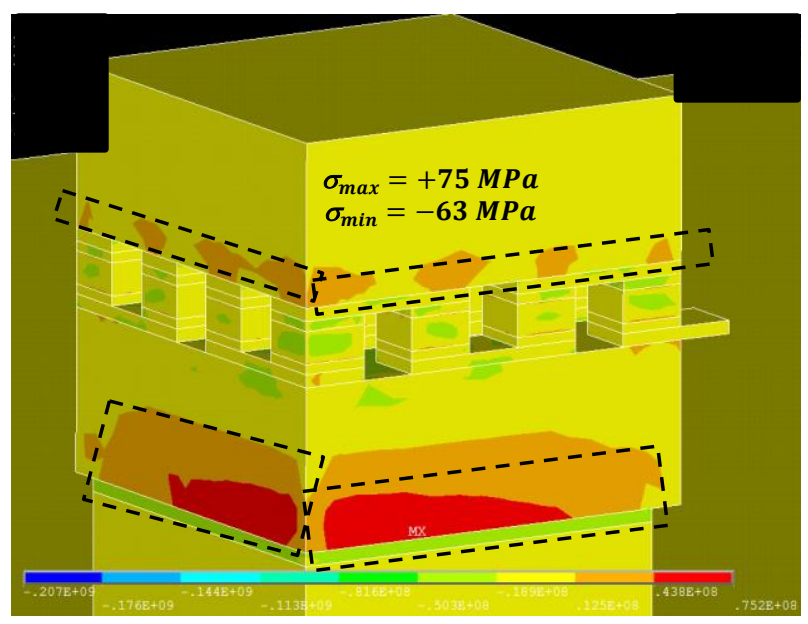

Figure 5.24: $2^{\text {nd }}$ Principal Stress Plot - TE Cooler Headers

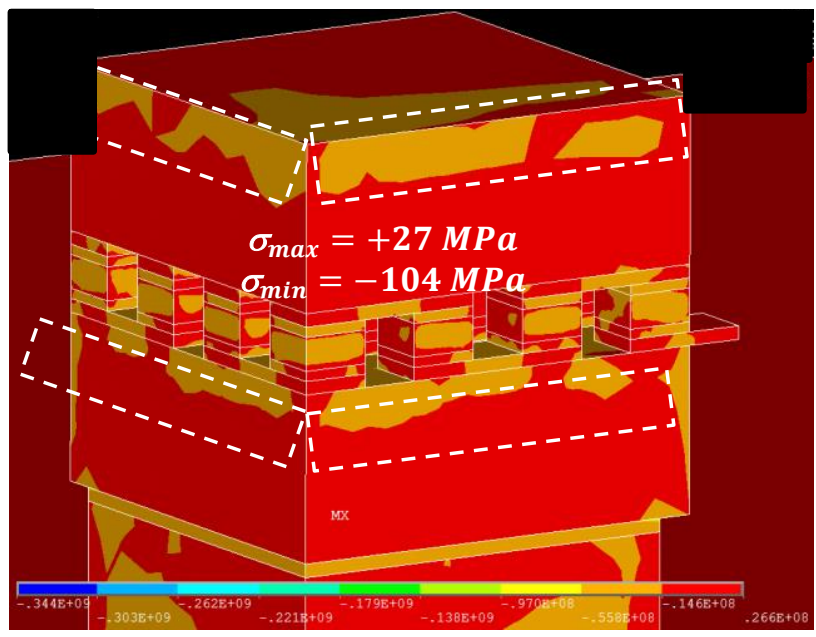

Figure 5.25: $3^{\text {rd }}$ Pr. Stress Plot - TE Cooler Headers 
Table 5.10: Principal Stresses - TE Cooler Header

\begin{tabular}{|c|c|c|c|}
\hline Parameter & $\begin{array}{c}\mathbf{1}^{\text {st }} \text { Principal } \\
\text { Stress }\end{array}$ & $\begin{array}{c}\mathbf{2}^{\text {nd }} \text { Principal } \\
\text { Stress }\end{array}$ & $\begin{array}{c}\mathbf{3}^{\text {rd }} \text { Principal } \\
\text { Stress }\end{array}$ \\
\hline Maximum & $\mathbf{1 4 0} \mathrm{MPa}$ & $75 \mathrm{MPa}$ & $27 \mathrm{MPa}$ \\
\hline Node Number & 107797 & 55864 & 132453 \\
\hline Minimum & $-48 \mathrm{MPa}$ & $-63 \mathrm{MPa}$ & $-104 \mathrm{MPa}$ \\
\hline Node Number & 109520 & 109520 & 115323 \\
\hline
\end{tabular}

Based on table 5.9 above, we can infer that a maximum principal stress of 140

MPa is seen in the header. The critical flaw size for this stress level was calculated as $76 \mu \mathrm{m}$ for $\mathrm{Y}=1.2, \mathrm{~K}_{\mathrm{IC}}=2.6 \mathrm{MPam}^{1 / 2}[51]$ and stress of $140 \mathrm{MPa}$. The flaw size is comparatively large and thus, the stress level is safe.

\subsubsection{Critical Flaw Size - GaN on SiC Device}

Table 5.11: Principal Stresses - GaN on SiC substrate

\begin{tabular}{|c|c|c|c|}
\hline Parameter & $\begin{array}{c}\mathbf{1}^{\text {st }} \text { Principal } \\
\text { Stress }\end{array}$ & $\begin{array}{c}\mathbf{2}^{\text {nd }} \text { Principal } \\
\text { Stress }\end{array}$ & $\begin{array}{c}\mathbf{3}^{\text {rd }} \text { Principal } \\
\text { Stress }\end{array}$ \\
\hline Maximum & $111.69 \mathrm{MPa}$ & $61.62 \mathrm{MPa}$ & $0.45 \mathrm{MPa}$ \\
\hline Node Number & 178099 & 178099 & 178099 \\
\hline Minimum & $-33 \mathrm{MPa}$ & $-190 \mathrm{MPa}$ & $-290 \mathrm{MPa}$ \\
\hline Node Number & 2919 & 178441 & 2919 \\
\hline
\end{tabular}

The first, second and third principal stress plots are shown on next page.

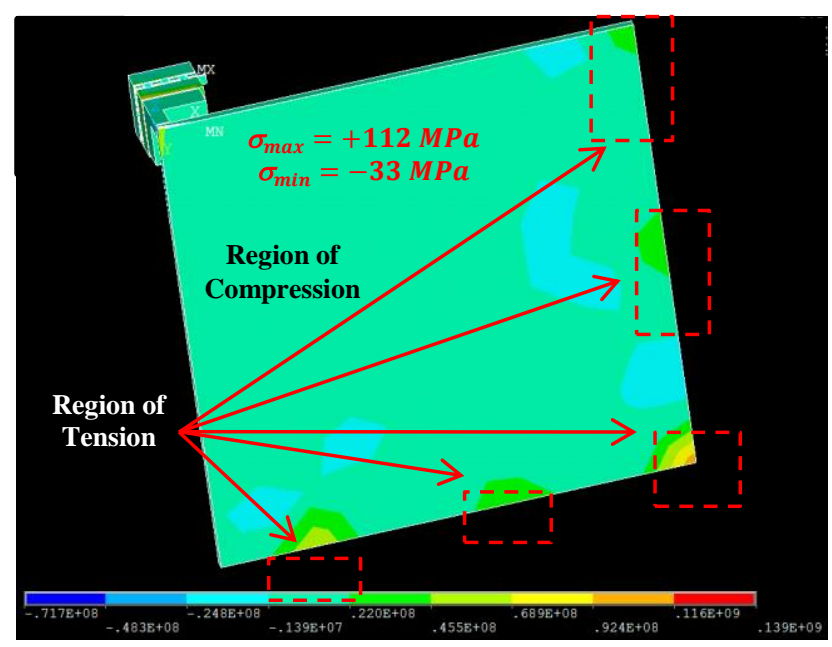

Figure 5.26: $1^{\text {st }}$ Principal Stress Plot - GaN on SiC Device 


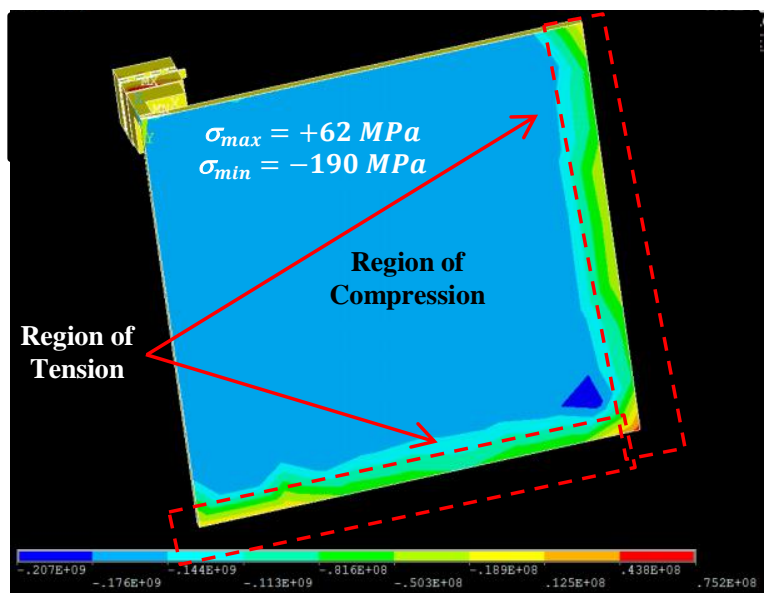

Figure 5.27: $2^{\text {nd }}$ Principal Stress Plot - GaN on SiC Device

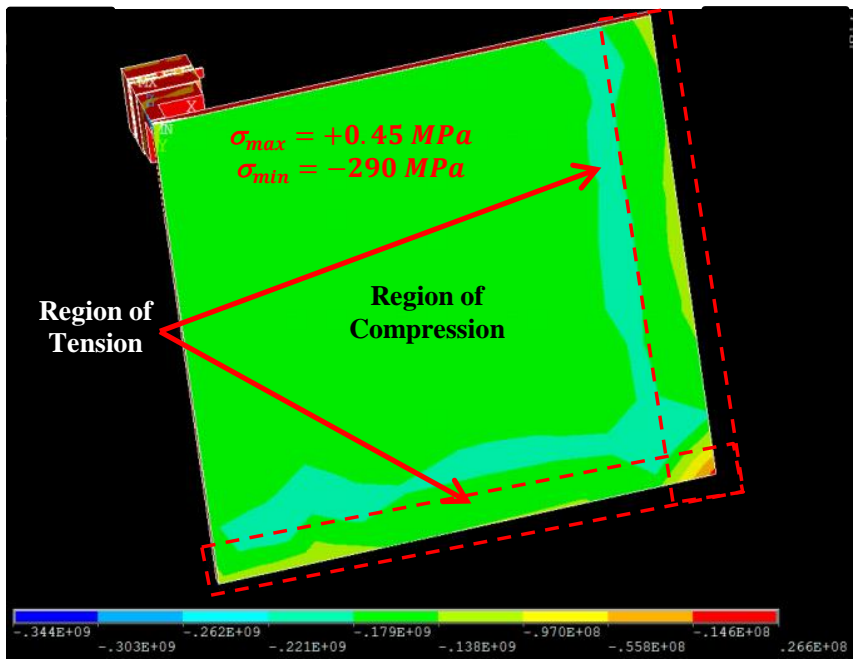

Figure 5.28: $3^{\text {rd }}$ Principal Stress Plot - GaN on SiC Device

From figures 5.26 - 5.28, a maximum principal stress of $112 \mathrm{MPa}$ was observed at the edge of $\mathrm{GaN}$ on $\mathrm{SiC}$ substrate (region of tension). Compressive stresses are seen at the center of device, near to the Mini-contact structure. The compressive stresses have a magnitude of -290 MPa and are supposed to affect the device performance by reverse piezoelectric effect. A critical flaw size of $21 \mu \mathrm{m}$ was calculated for a maximum principal stress of $112 \mathrm{MPa}$ based on fracture mechanics approach as shown earlier. 


\subsection{Discrete Mini-contact: Copper - Lofted Model}

\subsubsection{Temperature Plot}

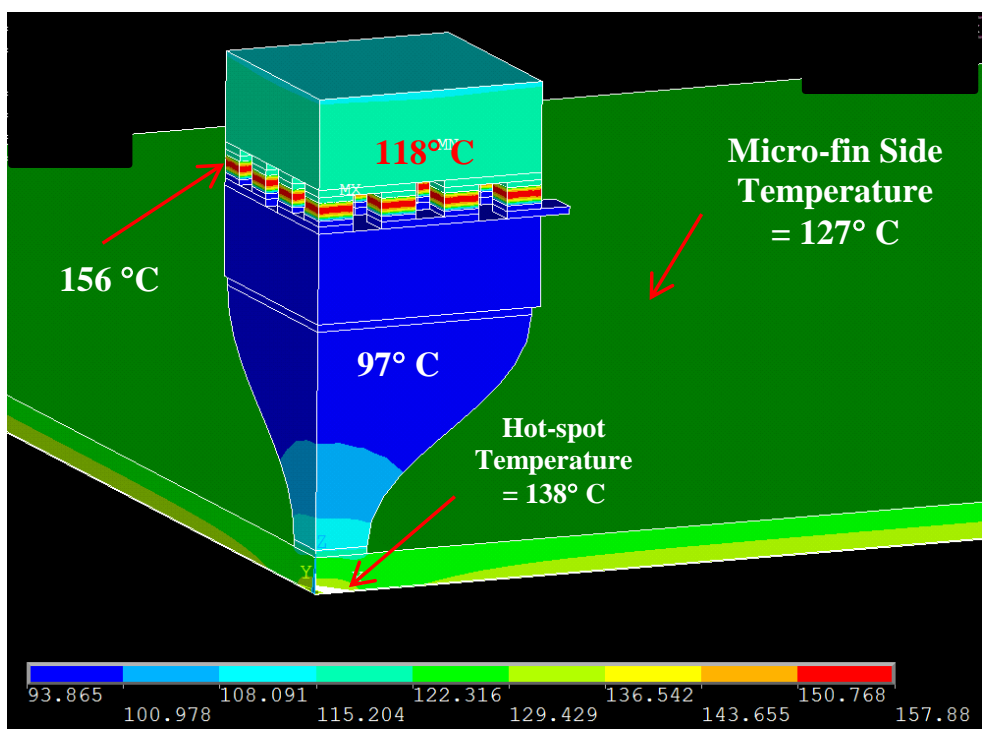

Figure 5.29: Temperature Contour Plot

The temperature contour across the electronics package is as shown above in figure 5.29. A maximum hot-spot temperature of $138^{\circ} \mathrm{C}$ was calculated through Finite Element simulations. The temperature on the micro-fin side is $127^{\circ} \mathrm{C}$. The corresponding displacements received in the structure are as shown below in figure 5.30 .

\subsubsection{Displacement Contour Plot}

The displacement contour plot is shown on next page. 


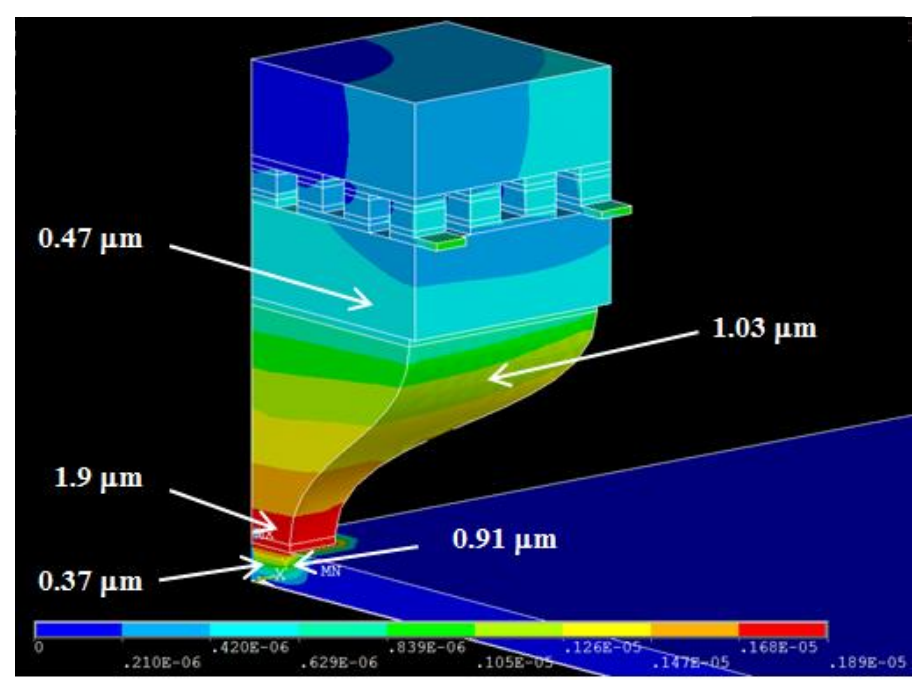

Figure 5.30: Displacement Contour Plot

A maximum displacement of $1.9 \mu \mathrm{m}$ was determined in the solder joint interconnecting the Mini-contact with $\mathrm{SiC}$ chip. A displacement in the range of 0.37 $\mu \mathrm{m}-0.91 \mu \mathrm{m}$ was seen in $\mathrm{SiC}$ substrate. Displacement of about $1 \mu \mathrm{m}$ was determined at top of Min-contact and $0.47 \mu \mathrm{m}$ in TE Cooler bottom header.

The $\mathrm{N}_{\mathrm{f}}(50 \%)$ for solder joint at chip level integration: 50,147 cycles, 117,958 cycles and 726,672 cycles. $\mathrm{N}_{\mathrm{f}}(63.2 \%): 56,656$ cycles, 133,269 cycles and 820,994 cycles. For contact/cooler integration level, $\mathrm{N}_{\mathrm{f}}(50 \%)$ : 143 cycles, 220 cycles and 554 cycles. $\mathrm{N}_{\mathrm{f}}(63.2 \%)$ : 161 cycles, 248 cycles and 625 cycles. MTTF values for the above integration levels are mentioned below in tables 5.11 and 5.12.

\subsubsection{Von-Mises Plastic Strain Plot}

The Von-Mises plastic strain plot is shown on next page. 


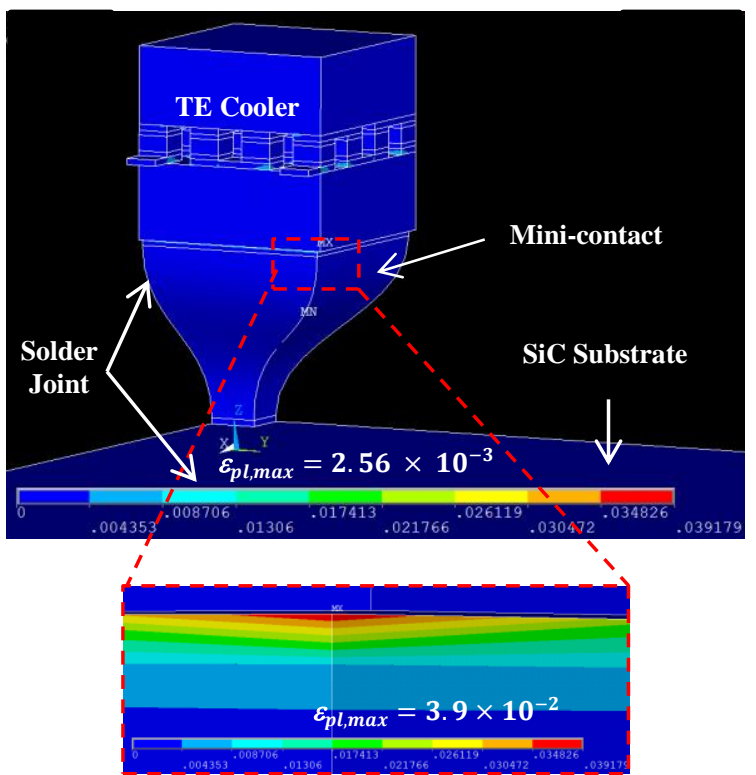

Figure 5.31: Pl. Strain - Solder Joint: Mini-contact/TE Cooler

Figure 5.31 gives Von-Mises plastic strain in SAC305 solder joint for chip level integration and Mini-contact/TE Cooler integration. As it can be inferred from the diagram, a Von-Mises plastic strain of magnitude $2.56 \times 10^{-3}$ and $3.9 \times 10^{-2}$ is respectively seen at the two levels. The design for reliability (MTTF) predictions for these plastic strain values are calculated based on the Engelmaier's model as shown in tables 5.11 and 5.12 below.

Table 5.12: Design for Reliability (MTTF) - Chip Level Integration

\begin{tabular}{|c|c|}
\hline Maximum Plastic Strain & $2.56 \mathrm{e}-3$ \\
\hline Maximum Temperature & $120^{\circ} \mathrm{C}$ \\
\hline Minimum Temperature & $25^{\circ} \mathrm{C}$ \\
\hline Average Temperature & $73^{\circ} \mathrm{C}$ \\
\hline Node Number & 24376 \\
\hline MTTF (596 mins.) & $\mathbf{3 8 , 5 9 4}$ cycles \\
\hline MTTF (20 mins.) & $\mathbf{1 1 7 , 9 5 8}$ cycles \\
\hline MTTF (1 min.) & $\mathbf{7 2 6 , 6 7 2}$ cycles \\
\hline
\end{tabular}


Table 5.13: Design for Reliability (MTTF) - Contact/Cooler Integration

\begin{tabular}{|c|c|}
\hline Maximum Plastic Strain & $3.92 \mathrm{e}-2$ \\
\hline Maximum Temperature & $95.43^{\circ} \mathrm{C}$ \\
\hline Minimum Temperature & $25^{\circ} \mathrm{C}$ \\
\hline Average Temperature & $60.21^{\circ} \mathrm{C}$ \\
\hline Node Number & 55762 \\
\hline MTTF (596 mins.) & $\mathbf{1 1 0}$ cycles \\
\hline MTTF (20 mins.) & $\mathbf{1 6 9}$ cycles \\
\hline MTTF (1 min.) & $\mathbf{4 2 6}$ cycles \\
\hline
\end{tabular}

As per the above tables 5.11 and 5.12, a MTTF of 38,594 cycles, 117,958 cycles and 726,672 cycles was calculated for solder joint at chip level integration, which interconnects Copper Mini-contact with SiC chip (plastic strain of $2.56 \times 10^{-3}$ and solder joint temperature of $120^{\circ} \mathrm{C}$ ). The MTTF for solder joint interconnecting Mini-contact with TE cooler was calculated as 110 cycles, 169 cycles and 426 cycles for a plastic strain value of $3.92 \times 10^{-2}$ and solder joint temperature of $95^{\circ} \mathrm{C}$.

The plastic strain of solder joint inside the TE cooler was calculated by the FE software $8.96 \times 10^{-3}$. As mentioned for earlier cases, the solder joint inter-connecting thermo-electric elements with metallized Copper on AlN headers on top side is under higher plastic strain due to higher temperature. The same is shown with the help of a schematic, figure 5.32 below.

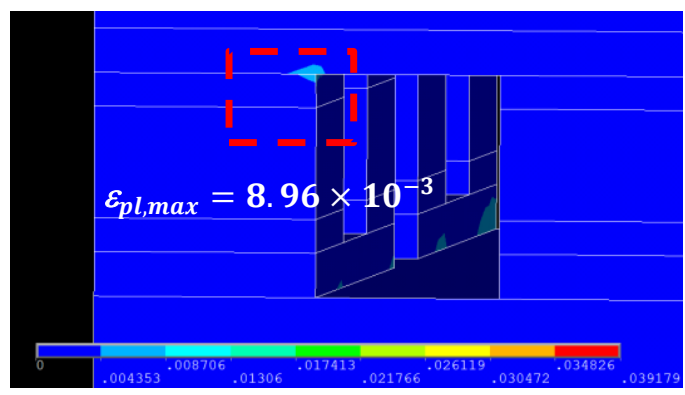

Figure 5.32: Pl. Strain - Solder Joint inside Thermo-electric Cooler 
The design for reliability (MTTF) prediction for the solder joint shown above in figure 5.32 was calculated based on Engelmaier's model as shown in table 5.13 below. $\mathrm{N}_{\mathrm{f}}(50 \%)$ : 3,131 cycles, 5,992 cycles and 23,745 cycles. The $\mathrm{N}_{\mathrm{f}}(63.2 \%)$ : 3,537 cycles, 5,992 cycles and 23,745 cycles.

Table 5.14: Design for Reliability (MTTF) - Solder joint inside TE Cooler

\begin{tabular}{|c|c|}
\hline Maximum Plastic Strain & $8.96 \mathrm{e}-3$ \\
\hline Maximum Temperature & $121.9^{\circ} \mathrm{C}$ \\
\hline Minimum Temperature & $25^{\circ} \mathrm{C}$ \\
\hline Average Temperature & $73.45^{\circ} \mathrm{C}$ \\
\hline Node Number & 124789 \\
\hline MTTF (596 mins.) & $\mathbf{2 , 7 9 8}$ cycles \\
\hline MTTF (20 mins.) & $\mathbf{4 , 6 1 2}$ cycles \\
\hline MTTF (1 min.) & $\mathbf{1 8 , 2 7 5}$ cycles \\
\hline
\end{tabular}

Based on table 5.13 shown above, the MTTF for the solder joint interconnecting thermo-electric elements with metallized Copper on AlN headers was calculated to be 2,798 cycles, 4,612 cycles and 18,275 cycles depending upon the dwell time. The MTTF of solder joint interconnecting to lower header was found to be 5680 cycles, mainly due to lower strains and temperature.

\subsubsection{Critical Flaw Size - Thermo-electric Elements}

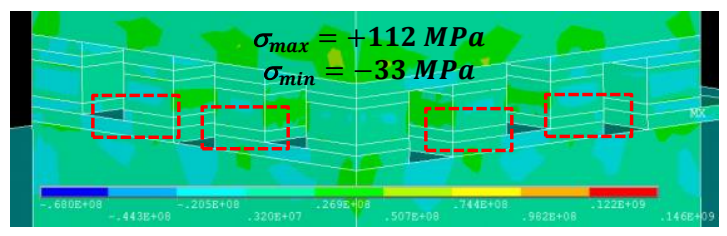

Figure 5.33: $1^{\text {st }}$ Principal Stress Plot - TE Elements

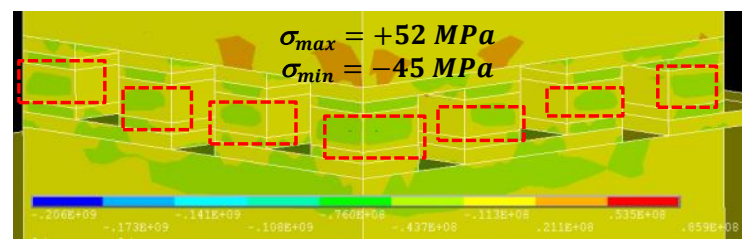

Figure 5.34: $2^{\text {nd }}$ Principal Stress Plot - TE Elements 


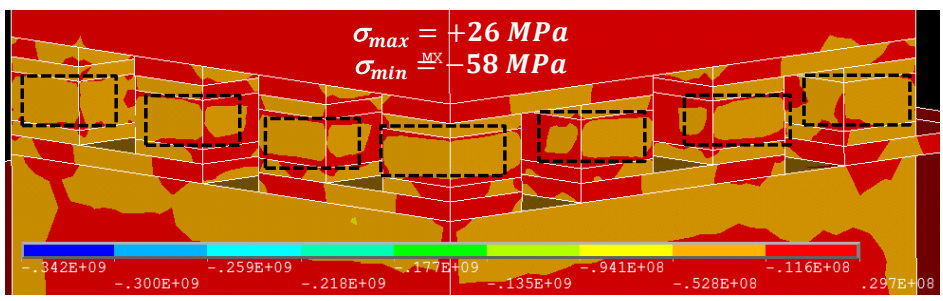

Figure 5.35: $3^{\text {rd }}$ Principal Stress Plot - TE Elements

Table 5.15: Principal Stresses - Thermoelectric Elements

\begin{tabular}{|c|c|c|c|}
\hline Parameter & $\begin{array}{c}\mathbf{1}^{\text {st }} \text { Principal } \\
\text { Stress }\end{array}$ & $\begin{array}{c}\mathbf{2}^{\text {nd }} \text { Principal } \\
\text { Stress }\end{array}$ & $\begin{array}{c}\mathbf{3}^{\text {rd }} \text { Principal } \\
\text { Stress }\end{array}$ \\
\hline Maximum & $77 \mathrm{MPa}$ & $52 \mathrm{MPa}$ & $26 \mathrm{MPa}$ \\
\hline Node Number & 103738 & 96226 & 103364 \\
\hline Minimum & $-27 \mathrm{MPa}$ & $-45 \mathrm{MPa}$ & $-58 \mathrm{MPa}$ \\
\hline Node Number & 96226 & 104063 & 96228 \\
\hline
\end{tabular}

The thermo-electric elements undergo a maximum principal stress of $77 \mathrm{MPa}$.

The critical flaw size for the corresponding stress can be calculated using the fracture mechanics approach. For $\mathrm{Y}=1.2, \mathrm{~K}_{\mathrm{IC}}=1 \mathrm{MPam}^{1 / 2}-2 \mathrm{MPam}^{1 / 2}$, the flaw size was calculated to be in the range of $37 \mu \mathrm{m}-149 \mu \mathrm{m}$. Considering the size of thermoelectric element, the flaw size is relatively large, and thus, the element is under safe stress levels.

Table 5.16: Principal Stresses - Thermoelectric Cooler Headers

\begin{tabular}{|c|c|c|c|}
\hline Parameter & $\begin{array}{c}\mathbf{1}^{\text {st }} \text { Principal } \\
\text { Stress }\end{array}$ & $\begin{array}{c}\mathbf{2}^{\text {nd }} \text { Principal } \\
\text { Stress }\end{array}$ & $\begin{array}{c}\mathbf{3}^{\text {rd }} \text { Principal } \\
\text { Stress }\end{array}$ \\
\hline Maximum & $146 \mathrm{MPa}$ & $86 \mathrm{MPa}$ & $30 \mathrm{MPa}$ \\
\hline Node Number & 116491 & 56166 & 168850 \\
\hline Minimum & $-48 \mathrm{MPa}$ & $-65 \mathrm{MPa}$ & $-104 \mathrm{MPa}$ \\
\hline Node Number & 118214 & 55868 & 124017 \\
\hline
\end{tabular}




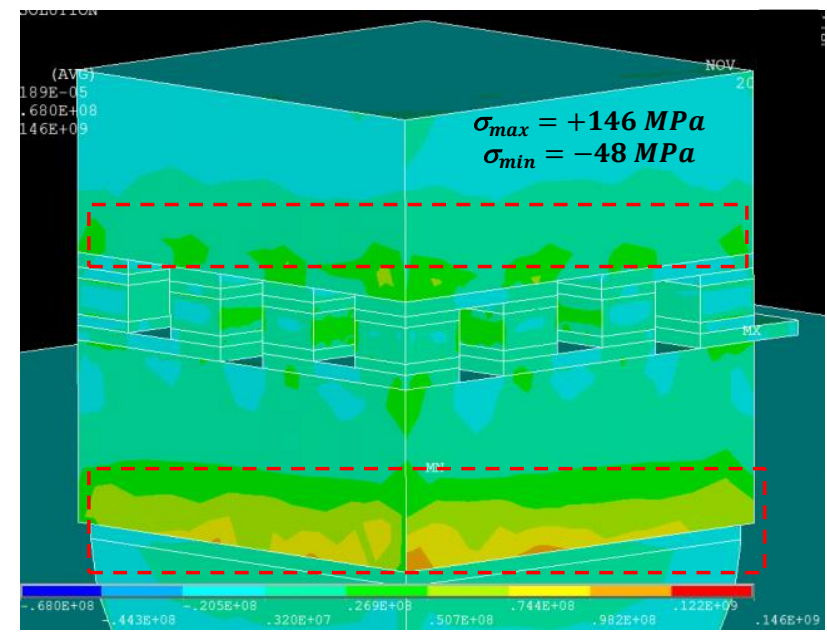

Figure 5.36: $1^{\text {st }}$ Principal Stress Plot - TE Cooler Headers

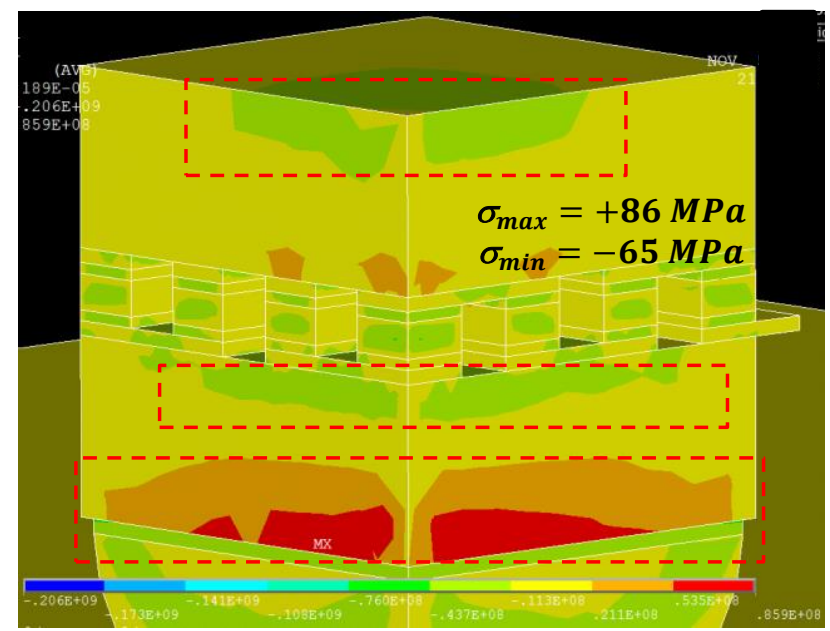

Figure 5.37: $2^{\text {nd }}$ Principal Stress Plot - TE Cooler Headers

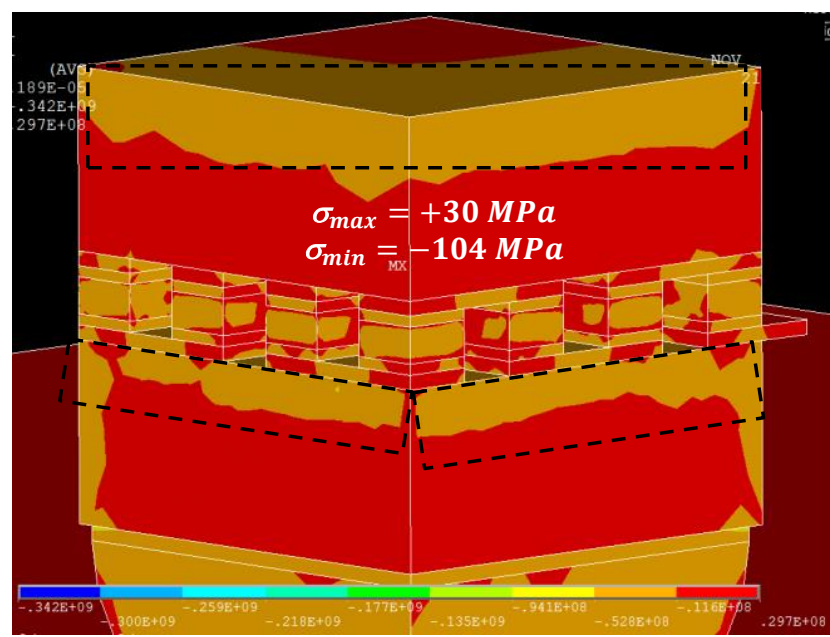

Figure 5.38: $3^{\text {rd }}$ Principal Stress Plot - TE Cooler Headers 
A maximum principal stress of $146 \mathrm{MPa}$ was seen in the AlN headers. The critical flaw size for this stress level was calculated as $70 \mu \mathrm{m}$ based on $\mathrm{Y}=1.2, \mathrm{~K}_{\mathrm{IC}}$ of 2.6 $\mathrm{MPam}^{1 / 2}[51]$ and stress of $146 \mathrm{MPa}$. This calculation is based on the fracture mechanics approach, where the stress concentrations on the other edge of material will assist in crack propagation. The crack size is relatively large in size though.

\subsubsection{Critical Flaw Size - GaN on $\mathrm{SiC}$ substrate}

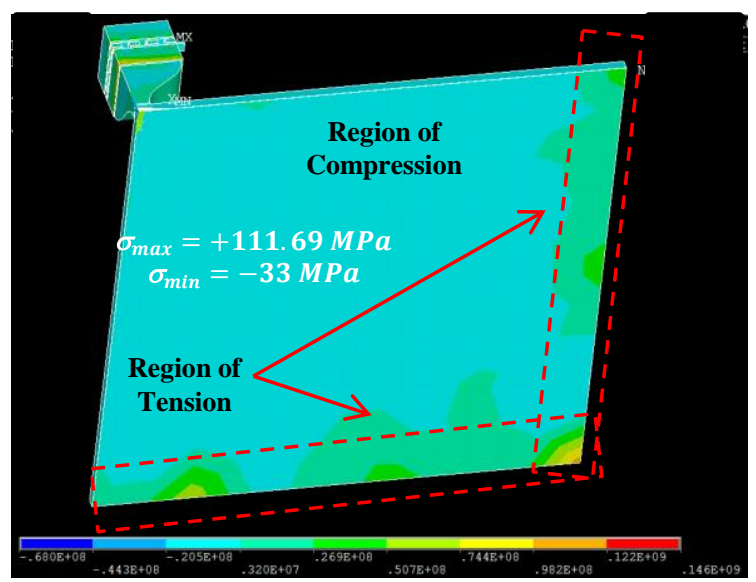

Figure 5.39: $1^{\text {st }}$ Principal Stress Plot - GaN on SiC

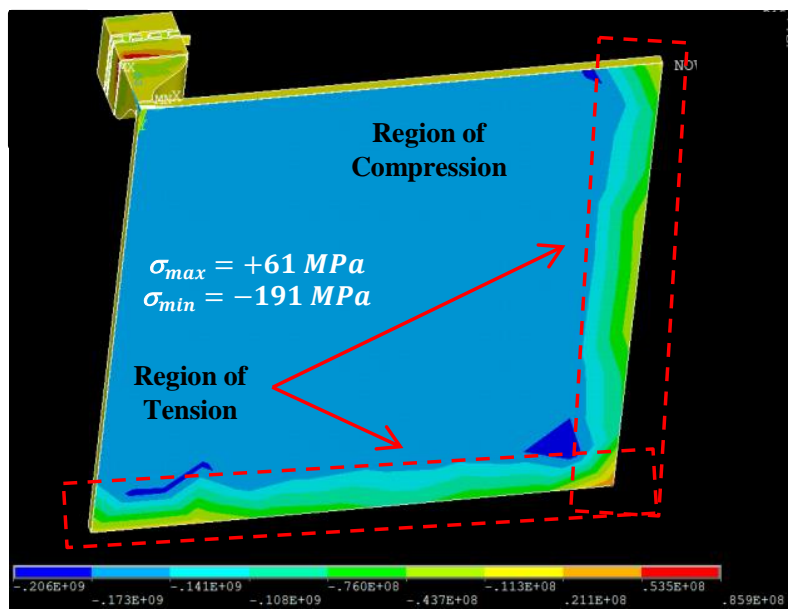

Figure 5.40: $2^{\text {nd }}$ Principal Stress Plot $-\mathrm{GaN}$ on $\mathrm{SiC}$ 


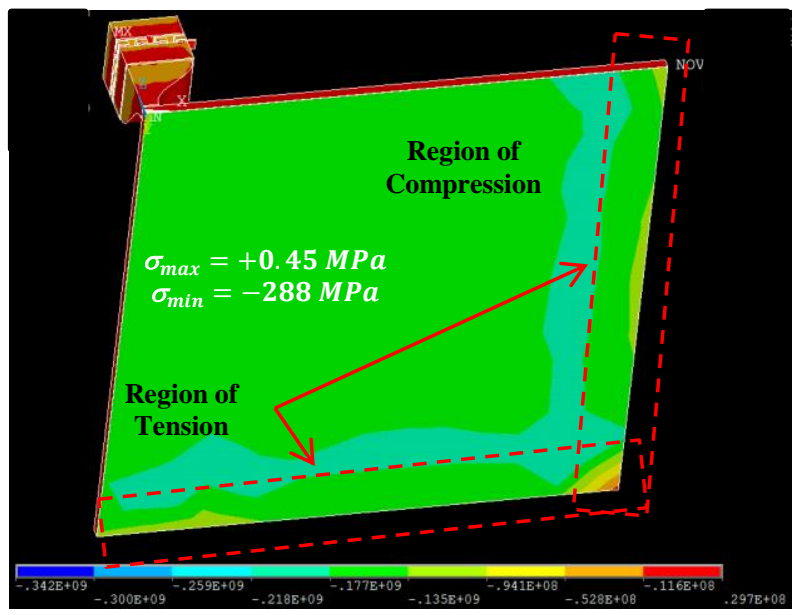

Figure 5.41: $3^{\text {rd }}$ Principal Stress Plot $-\mathrm{GaN}$ on SiC

Table 5.17: Principal Stresses - GaN on $\mathrm{SiC}$

\begin{tabular}{|c|c|c|c|}
\hline Parameter & $\begin{array}{c}\mathbf{1}^{\text {st }} \text { Principal } \\
\text { Stress }\end{array}$ & $\begin{array}{c}\mathbf{2}^{\text {nd }} \text { Principal } \\
\text { Stress }\end{array}$ & $\begin{array}{c}\mathbf{3}^{\text {rd }} \text { Principal } \\
\text { Stress }\end{array}$ \\
\hline Maximum & $\mathbf{1 1 2} \mathbf{M P a}$ & $61 \mathrm{MPa}$ & $0.45 \mathrm{MPa}$ \\
\hline $\begin{array}{c}\text { Node } \\
\text { Number }\end{array}$ & 185126 & 185126 & 185126 \\
\hline Minimum & $-33 \mathrm{MPa}$ & $-191 \mathrm{MPa}$ & $\mathbf{- 2 8 8} \mathbf{M P a}$ \\
\hline $\begin{array}{c}\text { Node } \\
\text { Number }\end{array}$ & 2919 & 185468 & 2919 \\
\hline
\end{tabular}

A maximum principal stress of $112 \mathrm{MPa}$ was seen in GaN device. The critical

flaw size was calculated to be $21 \mu \mathrm{m}$ based on fracture mechanics approach and assuming that $\mathrm{Y}=1.2$, stress $=112 \mathrm{MPa}, \mathrm{K}_{\mathrm{IC}}=1.1 \mathrm{MPam}^{1 / 2}$. There are compressive stresses at the center of $\mathrm{GaN}$ device, which are expected to affect the device performance by reverse piezoelectric effect. 


\subsection{Discrete Mini-contact: Copper - Taper Model}

5.4.1 Temperature Plot

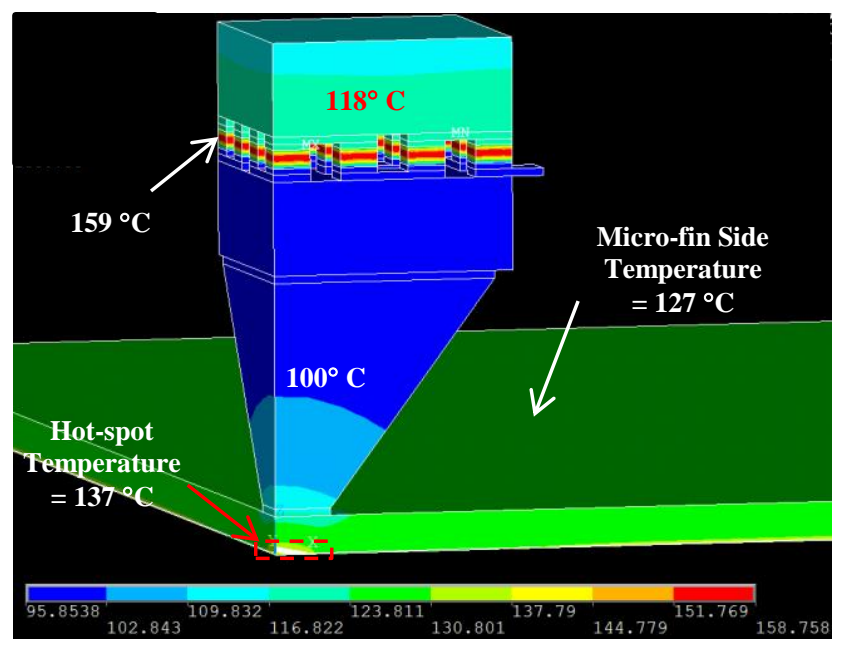

Figure 5.42: Temperature Contour Plot

The hot-spot has a maximum temperature of $137^{\circ} \mathrm{C}$ and the temperature on

micro-fin side is $127^{\circ} \mathrm{C}$. The hottest region in the package is thermo-electrics, which have a temperature of $159^{\circ} \mathrm{C}$.

\subsubsection{Displacement Contour Plot}

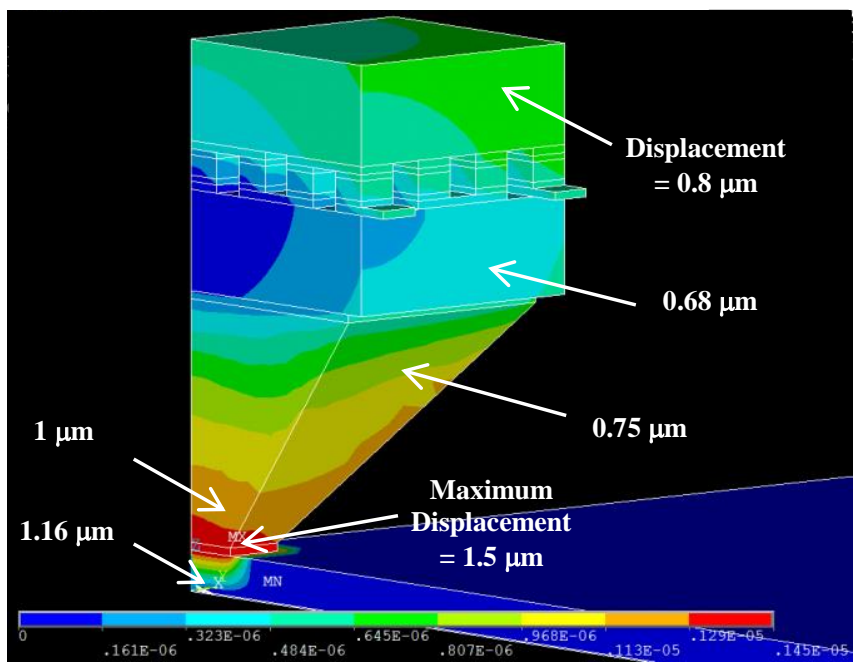

Figure 5.43: Displacement Contour Plot 
As shown in figure 5.43 above and previous cases of Copper Mini-contact (Tshaped and Lofted models), we see that the point of maximum deformation is solder joint interconnecting the taper Mini-contact with SiC chip. A maximum deformation of $1.5 \mu \mathrm{m}$ is seen in the solder joint, near the $\mathrm{SiC}$ region.

For dwell time of 596 mins., 20 mins. and 1 min., $\mathrm{N}_{\mathrm{f}}(50 \%)$ for solder joint at chip level integration: 20,850 cycles, 46,070 cycles and 251,808 cycles. $\mathrm{N}_{\mathrm{f}}(63.2 \%)$ : 23,566 cycles, 52,049 cycles and 284,493 cycles. At the next level of integration, which is Contact/Cooler integration, $\mathrm{N}_{\mathrm{f}}(50 \%)$ : 400 cycles, 665 cycles and 1,974 cycles. $\mathrm{N}_{\mathrm{f}}(63.2 \%): 452$ cycles, 751 cycles and 2,231 cycles. MTTF values are given as shown below in tables in 5.17 and 5.18.

\subsubsection{Von-Mises Plastic Strain Plot}

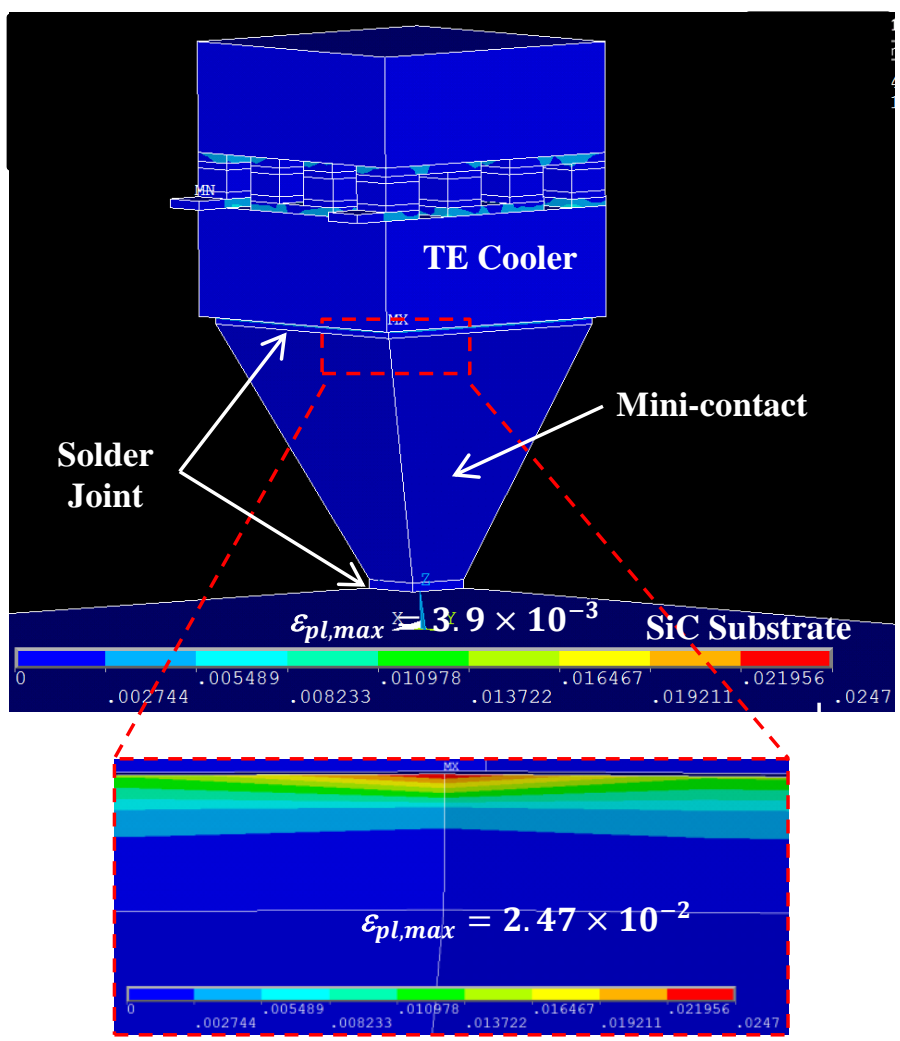

Figure 5.44: Pl. Strain - Solder Joint: Mini-contact/TE Cooler 
As shown in figure 5.44 above, a maximum plastic strain of $3.9 \times 10^{-3}$ is seen in the solder joint interconnecting taper Mini-contact with SiC substrate. There is a comparatively larger strain in solder joint at Mini-contact/Cooler interface. The magnitude for same is $2.47 \times 10^{-2}$. Copper assists SAC305 with plastic deformation since it's a very compliant ductile material. The reasoning for failure site is explained further in next Chapter 6.

The design for reliability (MTTF) predictions for the plastic strains above is calculated as shown in tables 5.17 and 5.18 on next page.

Table 5.18: Design for Reliability (MTTF) - Chip Level Integration

\begin{tabular}{|c|c|}
\hline Maximum Plastic Strain & $3.99 \mathrm{e}-3$ \\
\hline Maximum Temperature & $111.42^{\circ} \mathrm{C}$ \\
\hline Minimum Temperature & $25^{\circ} \mathrm{C}$ \\
\hline Average Temperature & $68.21^{\circ} \mathrm{C}$ \\
\hline Node Number & 24010 \\
\hline MTTF (596 mins.) & $\mathbf{1 5 , 9 7 3 ~ c y c l e s ~}$ \\
\hline MTTF (20 mins.) & $\mathbf{3 5 , 4 5 6}$ cycles \\
\hline MTTF (1 min.) & $\mathbf{1 9 3 , 7 9 7 ~ c y c l e s ~}$ \\
\hline
\end{tabular}

Table 5.19: Design for Reliability (MTTF) - Mini-contact/Cooler Integration

\begin{tabular}{|c|c|}
\hline Maximum Plastic Strain & $2.47 \mathrm{e}-2$ \\
\hline Maximum Temperature & $97.35^{\circ} \mathrm{C}$ \\
\hline Minimum Temperature & $25^{\circ} \mathrm{C}$ \\
\hline Average Temperature & $61.17^{\circ} \mathrm{C}$ \\
\hline Node Number & 55762 \\
\hline MTTF (596 mins.) & $\mathbf{3 0 8}$ cycles \\
\hline MTTF (20 mins.) & $\mathbf{5 1 2}$ cycles \\
\hline MTTF (1 min.) & $\mathbf{1 , 5 1 9}$ cycles \\
\hline
\end{tabular}

The solder joint at chip level has a higher reliability as compared to that at Mini-contact/Cooler interface. The values for chip level integration are 15,973 cycles, 35,456 cycles and 193,797 cycles. At contact/cooler integration level, the MTTF was 
found out to be 308 cycles, 512 cycles and 1,519 cycles respectively. The plastic strain in solder joint inside the thermo-electric cooler is shown below in figure 5.45.

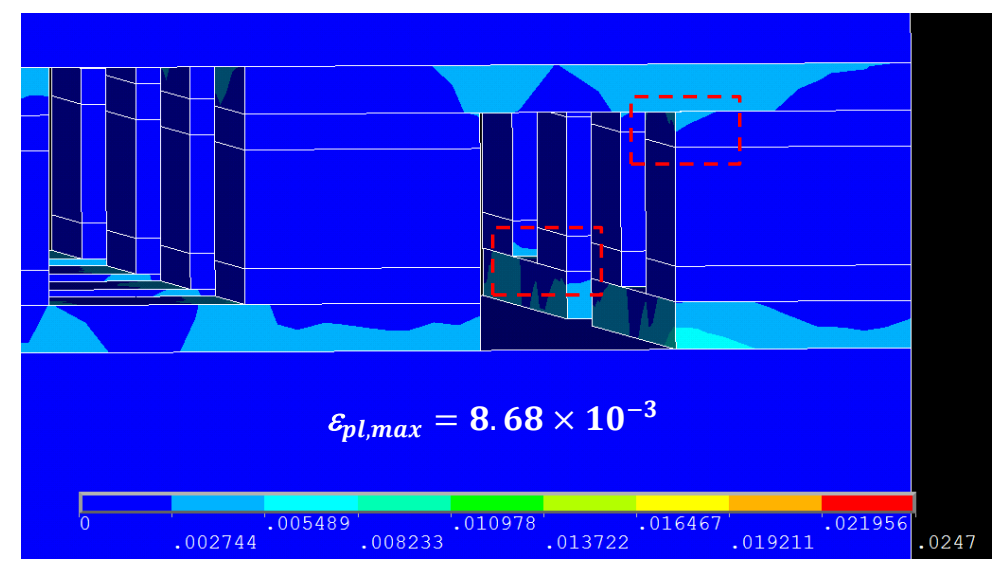

Figure 5.45: Pl. Strain - Solder joint inside TE Cooler

The thermo-electric cooler consists of a stack of layered materials. The solder joint interconnecting thermo-electric elements with metallized Copper deposited on headers on the top side fails first. The MTTF of solder for a plastic strain value of $8.68 \times 10^{-3}$ is calculated as shown below in table $5.19 . \mathrm{N}_{\mathrm{f}}(50 \%): 3,358$ cycles, 6,453 cycles and 25,850 cycles. $\mathrm{N}_{\mathrm{f}}(63.2 \%): 3,794$ cycles, 7,290 cycles and 29,205 cycles. The MTTF of solder joint is calculated as 2,584 cycles, 4,996 cycles and 19,895 cycles.

Table 5.20: Design for Reliability (MTTF) - Solder inside TE Cooler

\begin{tabular}{|c|c|}
\hline Maximum Plastic Strain & $8.68 \mathrm{e}-3$ \\
\hline Maximum Temperature & $121.97^{\circ} \mathrm{C}$ \\
\hline Minimum Temperature & $25^{\circ} \mathrm{C}$ \\
\hline Average Temperature & $73.49^{\circ} \mathrm{C}$ \\
\hline Node Number & 115370 \\
\hline MTTF (596 mins.) & $\mathbf{2 , 5 8 4}$ cycles \\
\hline MTTF (20 mins.) & $\mathbf{4 , 9 9 6 ~ c y c l e s ~}$ \\
\hline MTTF (1 min.) & $\mathbf{1 9 , 8 9 5}$ cycles \\
\hline
\end{tabular}




\subsubsection{Critical Flaw Size - Thermo-electric Elements and Headers}

In this section, the principal stresses received in thermo-electric elements and AlN headers will be plotted. Based on fracture mechanics approach, critical flaw size that can cause fracture of elements or header will be reported.

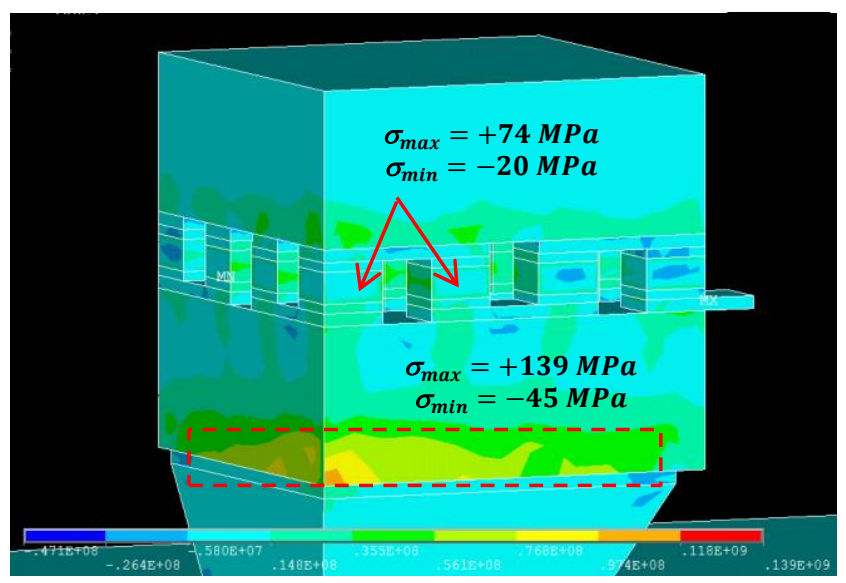

Figure 5.46: $1^{\text {st }}$ Principal Stress Plot - TE Elements and Headers

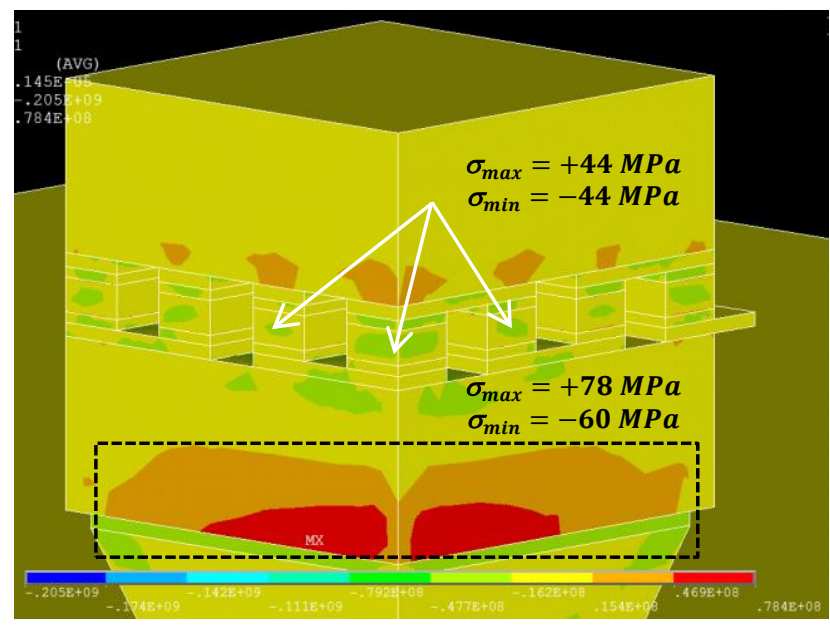

Figure 5.47: $2^{\text {nd }}$ Principal Stress Plot - TE Elements and Headers 


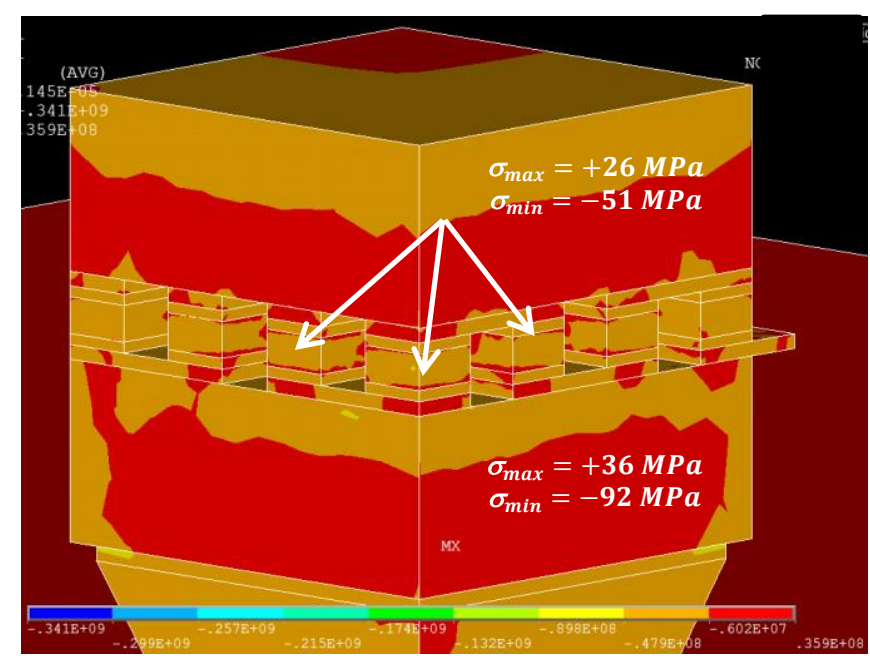

Figure 5.48: $3^{\text {rd }}$ Principal Stress Plot - TE Elements/Headers

Table 5.21: Principal Stresses - Thermoelectric Elements

\begin{tabular}{|c|c|c|c|}
\hline Parameter & $\begin{array}{c}\mathbf{1}^{\text {st }} \text { Principal } \\
\text { Stress }\end{array}$ & $\begin{array}{c}\mathbf{2}^{\text {nd }} \text { Principal } \\
\text { Stress }\end{array}$ & $\begin{array}{c}\mathbf{3}^{\text {rd }} \text { Principal } \\
\text { Stress }\end{array}$ \\
\hline Maximum & $74 \mathrm{MPa}$ & $44 \mathrm{MPa}$ & $26 \mathrm{MPa}$ \\
\hline Node Number & 94319 & 86807 & 93945 \\
\hline Minimum & $-20 \mathrm{MPa}$ & $-44 \mathrm{MPa}$ & $-51 \mathrm{MPa}$ \\
\hline Node Number & 86807 & 94644 & 94294 \\
\hline
\end{tabular}

The critical flaw size for a maximum stress of $74 \mathrm{MPa}$ in thermo-electric

elements was calculated to be in between $40 \mu \mathrm{m}$ - $162 \mu \mathrm{m}$ for a fracture toughness value of $1 \mathrm{MPam}^{1 / 2}-2 \mathrm{MPam}^{1 / 2}$ and $\mathrm{Y}=1.2$. This crack size is relatively large and thus, the thermo-electric elements are under safe stress.

Table 5.22: Principal Stresses - TE Cooler Headers

\begin{tabular}{|c|c|c|c|}
\hline Parameter & $\begin{array}{c}\mathbf{1}^{\text {st }} \text { Principal } \\
\text { Stress }\end{array}$ & $\begin{array}{c}\mathbf{2}^{\text {nd }} \text { Principal } \\
\text { Stress }\end{array}$ & $\begin{array}{c}\mathbf{3}^{\text {rd }} \text { Principal } \\
\text { Stress }\end{array}$ \\
\hline Maximum & $\mathbf{1 3 9} \mathrm{MPa}$ & $78 \mathrm{MPa}$ & $36 \mathrm{MPa}$ \\
\hline Node Number & 107072 & 56154 & 131728 \\
\hline Minimum & $-45 \mathrm{MPa}$ & $-60 \mathrm{MPa}$ & $-92 \mathrm{MPa}$ \\
\hline Node Number & 108795 & 113654 & 114598 \\
\hline
\end{tabular}


The critical flaw size for a maximum stress of $139 \mathrm{MPa}$ in AlN headers was calculated to be $77 \mu \mathrm{m}$ for a fracture toughness (AlN) value of $2.6 \mathrm{MPam}^{1 / 2}$ and $\mathrm{Y}=1.2$.

\subsubsection{Critical Flaw Size - GaN on SiC substrate}

The principal stresses in $\mathrm{GaN}$ device are shown in table 5.22 below.

Table 5.23: Principal Stresses - SiC/GaN Device

\begin{tabular}{|c|c|c|c|}
\hline Parameter & $\begin{array}{c}\mathbf{1}^{\text {st }} \text { Principal } \\
\text { Stress }\end{array}$ & $\begin{array}{c}\mathbf{2}^{\text {nd }} \text { Principal } \\
\text { Stress }\end{array}$ & $\begin{array}{c}\mathbf{3}^{\text {rd }} \text { Principal } \\
\text { Stress }\end{array}$ \\
\hline Maximum & $\mathbf{1 1 2} \mathbf{M P a}$ & $62 \mathrm{MPa}$ & $0.45 \mathrm{MPa}$ \\
\hline Node Number & 177469 & 177469 & 177469 \\
\hline Minimum & $-33 \mathrm{MPa}$ & $-191 \mathrm{MPa}$ & $-\mathbf{2 8 8} \mathrm{MPa}$ \\
\hline Node Number & 2919 & 177811 & 2919 \\
\hline
\end{tabular}

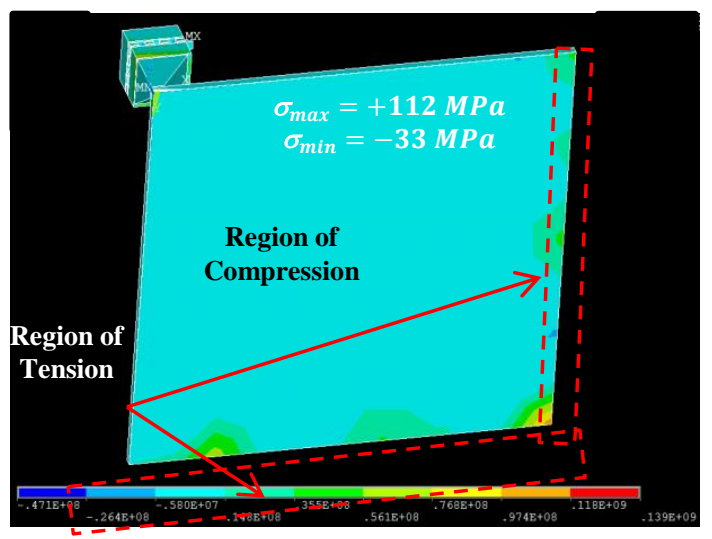

Figure 5.49: $1^{\text {st }}$ Principal Stress Plot - SiC/GaN Device

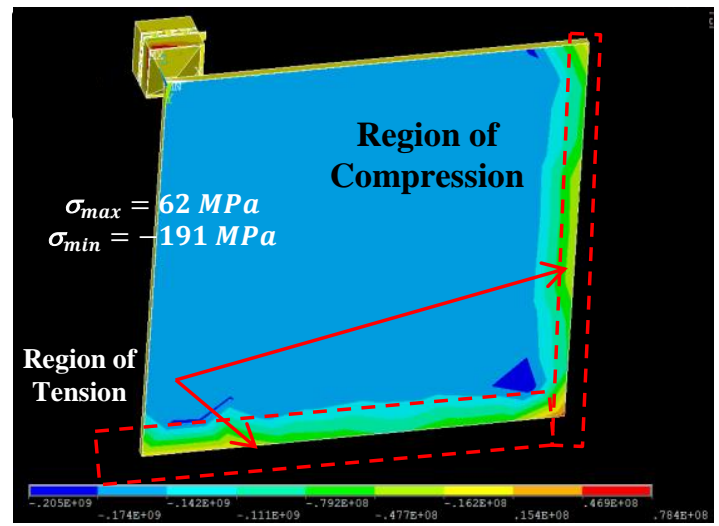

Figure 5.50: $2^{\text {nd }}$ Principal Stress Plot - SiC/GaN Device 


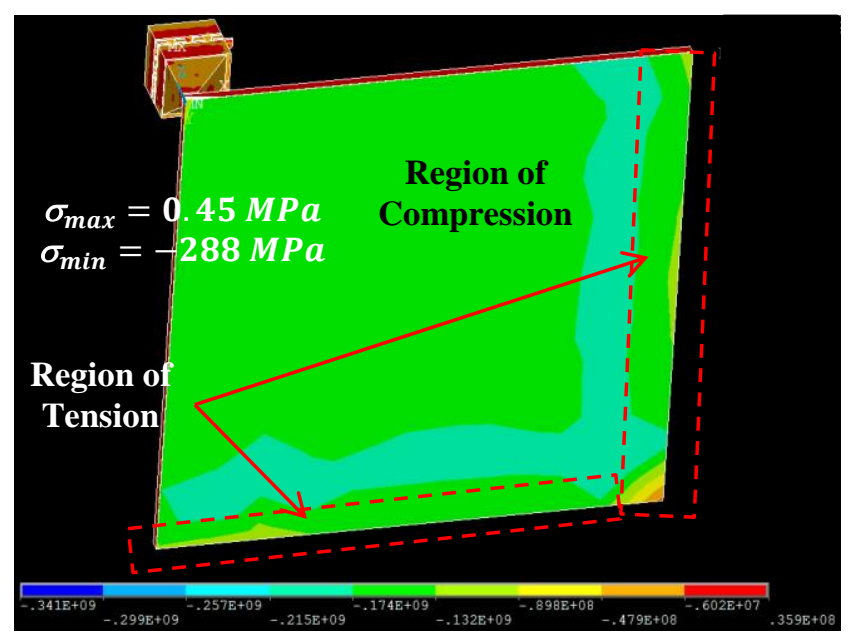

Figure 5.51: $3^{\text {rd }}$ Principal Stress Plot - SiC/GaN Device

For a maximum principal stress of about $112 \mathrm{MPa}$, the critical flaw size was calculated to be $21 \mu \mathrm{m}$ based on fracture mechanics principle $\left(\mathrm{Y}=1.2\right.$ and $\mathrm{K}_{\mathrm{IC}}=1.1$ $\mathrm{MPam}^{1 / 2}$ ). There are negative stresses (compressive) of magnitude -288 MPa, which may affect device performance by reverse piezoelectric effect. 


\subsection{Discrete Mini-contact: Diamond - T-shaped Model}

5.5.1 Nodal Temperature Plot

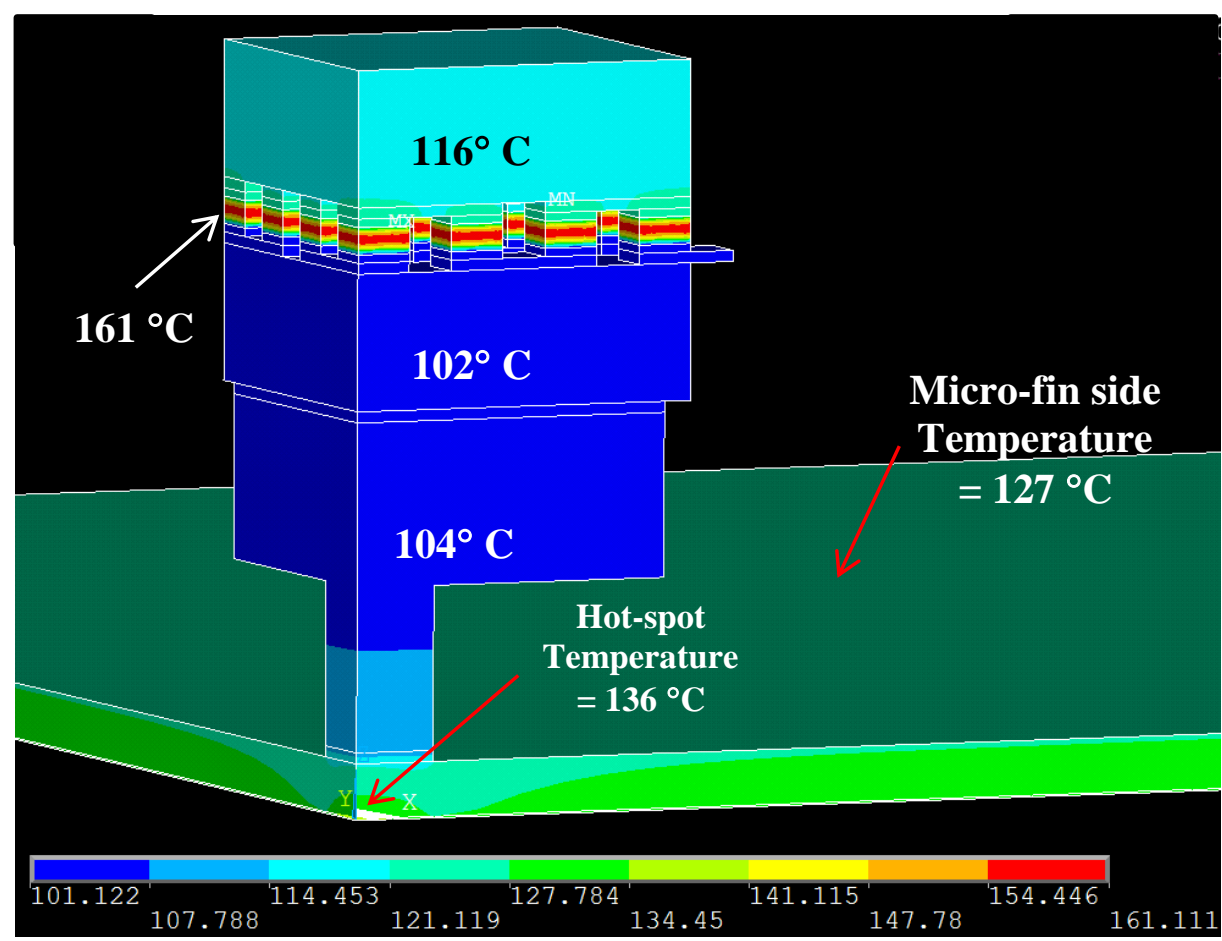

Figure 5.52: Temperature Contour Plot

Figure 5.52 gives the temperature contour plot across the 3D structure for a diamond Mini-contact. The diamond Mini-contact is interconnected to the SiC chip via SAC305 solder. A maximum hot-spot temperature of $136^{\circ} \mathrm{C}$ was determined through Finite Element (FE) simulations. The temperature on the micro-fin side was determined to be $127^{\circ} \mathrm{C}$ through modeling approach, which was found to be consistent for all different cases of Mini-contact integration. The hottest part on the device was thermo-electric elements with a temperature of $161^{\circ} \mathrm{C}$. 


\subsubsection{Displacement Contour Plot}

The displacement across the package is as shown in figure 5.53 below.

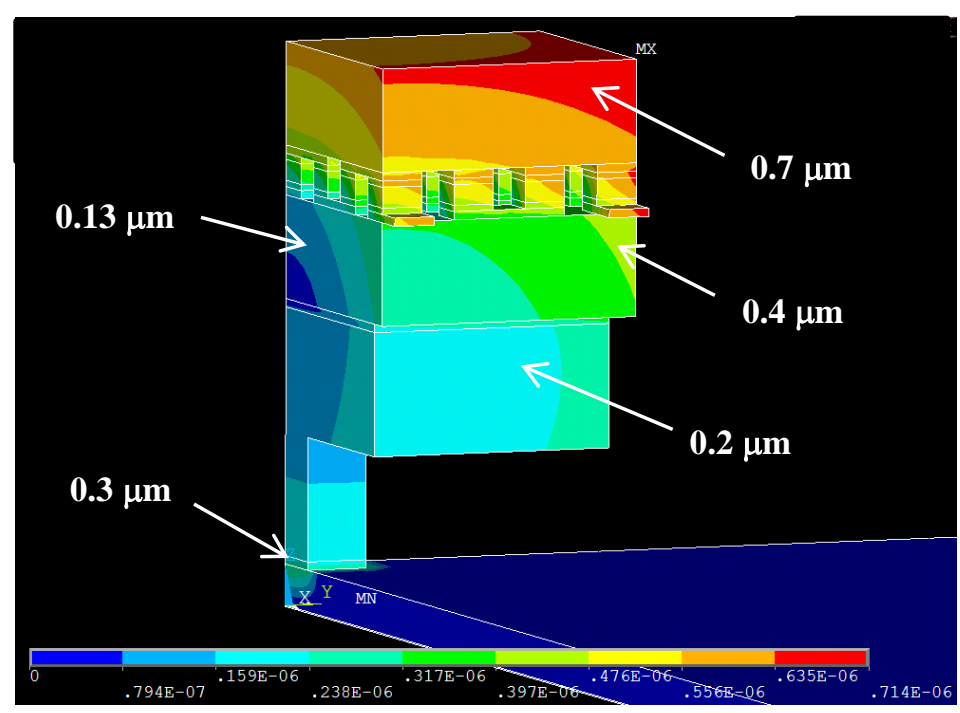

Figure 5.53: Displacement Contour Plot

Through finite element simulations, a maximum displacement of $0.7 \mu \mathrm{m}$ was determined at the top edge of the thermo-electric cooler. As compared to the Copper Mini-contact, the maximum deformation site has shifted to the edge of cooler. This is similar to the situation of an integrated SiC Mini-contact. Diamond has a C.T.E. of 1 $\mathrm{ppm} / \mathrm{K}$ and is highly elastic in nature, which shifts the maximum deformation site in thermo-electric cooler.

\subsubsection{Design for Reliability: Mean Time to Failure - MTTF}

The Mean Time to Failure (MTTF) of solder joint for three levels of integration will be presented in this section. In order to predict same, the plastic strain values were determined from thermo-mechanical finite element simulations. The plastic strain plots for the three cases are as shown in figures $5.54-5.56$. 


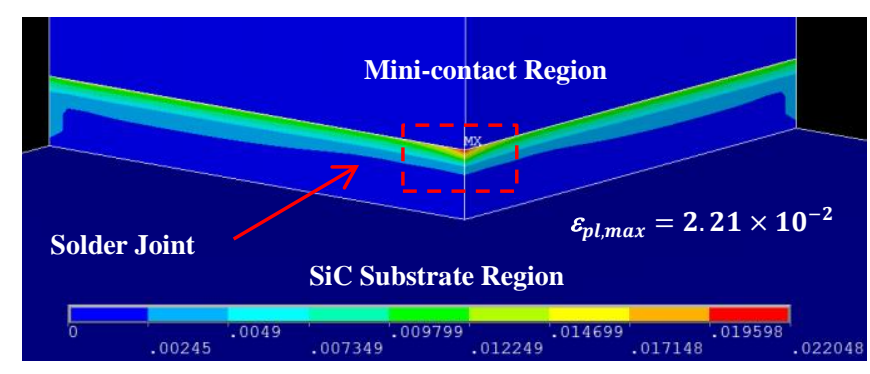

Figure 5.54: Pl. Strain - Solder Joint: Mini-contact/SiC Substrate Interface

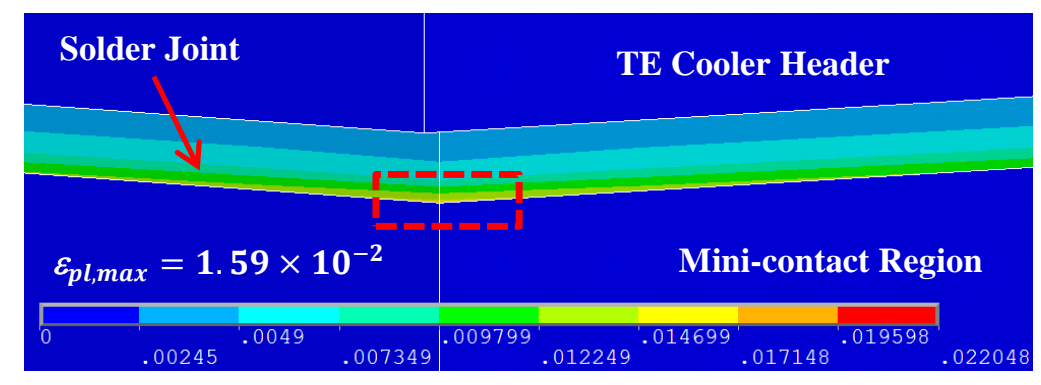

Figure 5.55: Pl. Strain - Solder Joint - Cooler/Mini-contact Integration

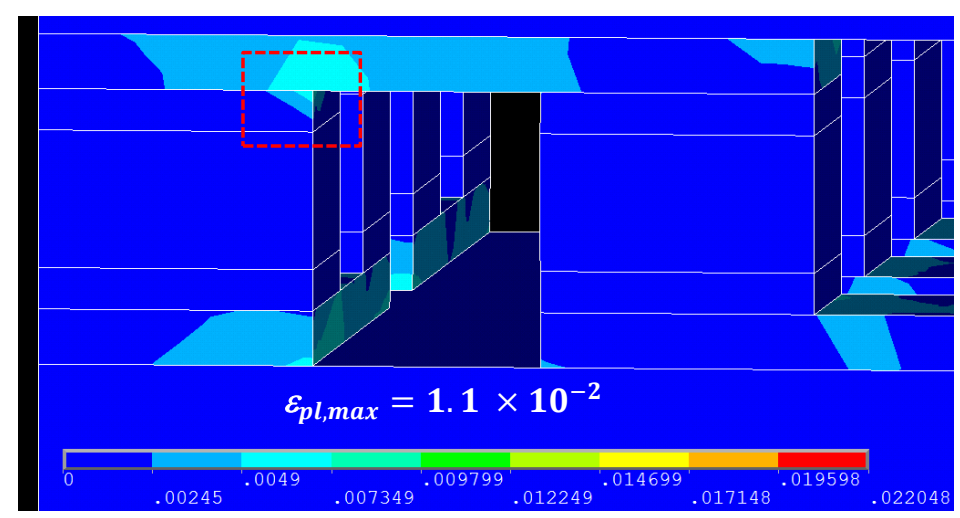

Figure 5.56: Pl. Strain - Solder Joint: Thermo-electric Cooler

The MTTF values for three cases were calculated as shown in tables 5.23 -

5.25 below. For chip level integration, $\mathrm{N}_{\mathrm{f}}(50 \%)$ : 471 cycles, 787 cycles and 2,353

cycles. $\mathrm{N}_{\mathrm{f}}(63.2 \%): 532$ cycles, 890 cycles and 2,658 cycles.

At the next level of integration, (Mini-contact/Cooler), $\mathrm{N}_{\mathrm{f}}(50 \%)$ : 471 cycles, 787 cycles and 2,353 cycles. $\mathrm{N}_{\mathrm{f}}(63.2 \%)$ : 532 cycles, 890 cycles and 2,658 cycles.

Inside TE Cooler, $\mathrm{N}_{\mathrm{f}}(50 \%)$ : 1916 cycles, 3,532 cycles and 12,947 cycles. $\mathrm{N}_{\mathrm{f}}$ (63.2\%): 2,165 cycles, 3,990 cycles and 14,627 cycles. 
Table 5.24: Design for Reliability (MTTF) - Chip Level Integration

\begin{tabular}{|c|c|}
\hline Maximum Plastic Strain & $2.20 \mathrm{e}-2$ \\
\hline Maximum Temperature & $110.80^{\circ} \mathrm{C}$ \\
\hline Minimum Temperature & $25^{\circ} \mathrm{C}$ \\
\hline Average Temperature & $67.90^{\circ} \mathrm{C}$ \\
\hline Node Number & 14324 \\
\hline MTTF $(\mathbf{5 9 6}$ mins.) & $\mathbf{3 6 3}$ cycles \\
\hline MTTF (20 mins.) & $\mathbf{6 0 6}$ cycles \\
\hline MTTF (1 min.) & $\mathbf{2 , 1 0 2 ~ c y c l e s ~}$ \\
\hline
\end{tabular}

Table 5.25: Design for Reliability (MTTF) - Mini-contact/Cooler Integration

\begin{tabular}{|c|c|}
\hline Maximum Plastic Strain & $1.59 \mathrm{e}-2$ \\
\hline Maximum Temperature & $103.87^{\circ} \mathrm{C}$ \\
\hline Minimum Temperature & $25^{\circ} \mathrm{C}$ \\
\hline Average Temperature & $64.435^{\circ} \mathrm{C}$ \\
\hline Node Number & 39753 \\
\hline MTTF $(\mathbf{5 9 6}$ mins.) & $\mathbf{9 1 4}$ cycles \\
\hline MTTF $(\mathbf{2 0}$ mins.) & $\mathbf{1 , 4 0 2}$ cycles \\
\hline MTTF (1 min.) & $\mathbf{4 , 7 9 7 ~ c y c l e s ~}$ \\
\hline
\end{tabular}

Table 5.26: Design for Reliability (MTTF) - Solder Joint: TE Cooler

\begin{tabular}{|c|c|}
\hline Maximum Plastic Strain & $1.12 \mathrm{e}-2$ \\
\hline Maximum Temperature & $122.13^{\circ} \mathrm{C}$ \\
\hline Minimum Temperature & $25^{\circ} \mathrm{C}$ \\
\hline Average Temperature & $73.565^{\circ} \mathrm{C}$ \\
\hline Node Number & 103282 \\
\hline MTTF $(\mathbf{5 9 6}$ mins.) & $\mathbf{1 , 7 1 1}$ cycles \\
\hline MTTF $(\mathbf{2 0}$ mins.) & $\mathbf{2 , 7 1 8 ~ c y c l e s ~}$ \\
\hline MTTF $(\mathbf{1}$ min.) & $\mathbf{9 , 9 6 4}$ cycles \\
\hline
\end{tabular}

From tables above, we can conclude that the failure site shifts to solder joint at chip level integration. The MTTF of solder interconnecting Mini-contact with SiC chip was calculated as 363 cycles, 606 cycles and 2,102 cycles. MTTF of solder joint at Mini-contact/Cooler integration was calculated as 914 cycles, 1,402 cycles and 4,797 cycles. MTTF of solder joint inside the cooler: 1,711 cycles, 2,718 cycles and 9,964 cycles. 
5.5.4 Maximum Principal Stress Plot - Diamond Mini-contact

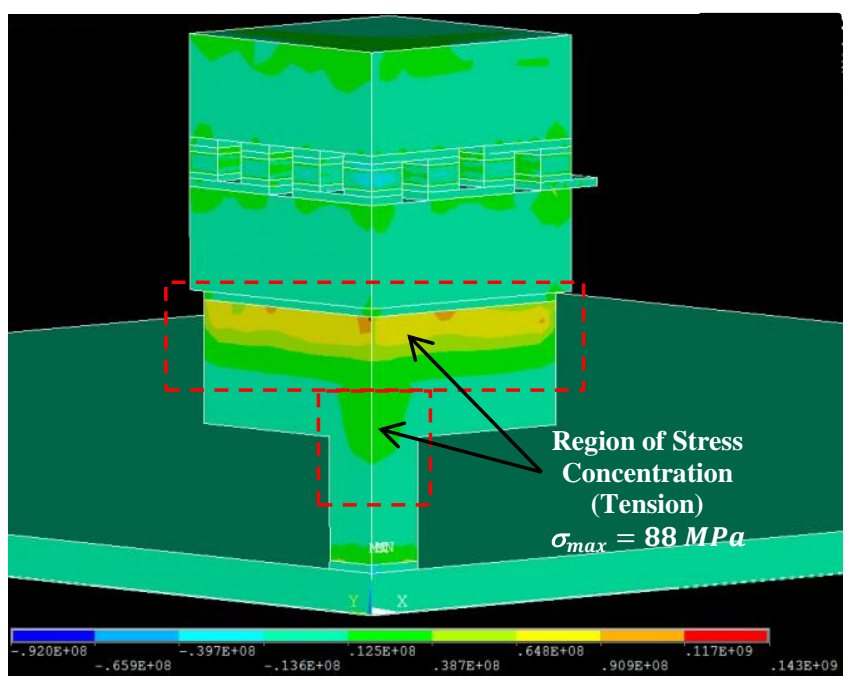

Figure 5.57: ${ }^{\text {st }}$ Principal Stress Plot 1 - Diamond Mini-contact Region

Diamond is a linear elastic material and is known to fail by brittle fracture.

Thus, it's important to determine if structure will fail by cracking.

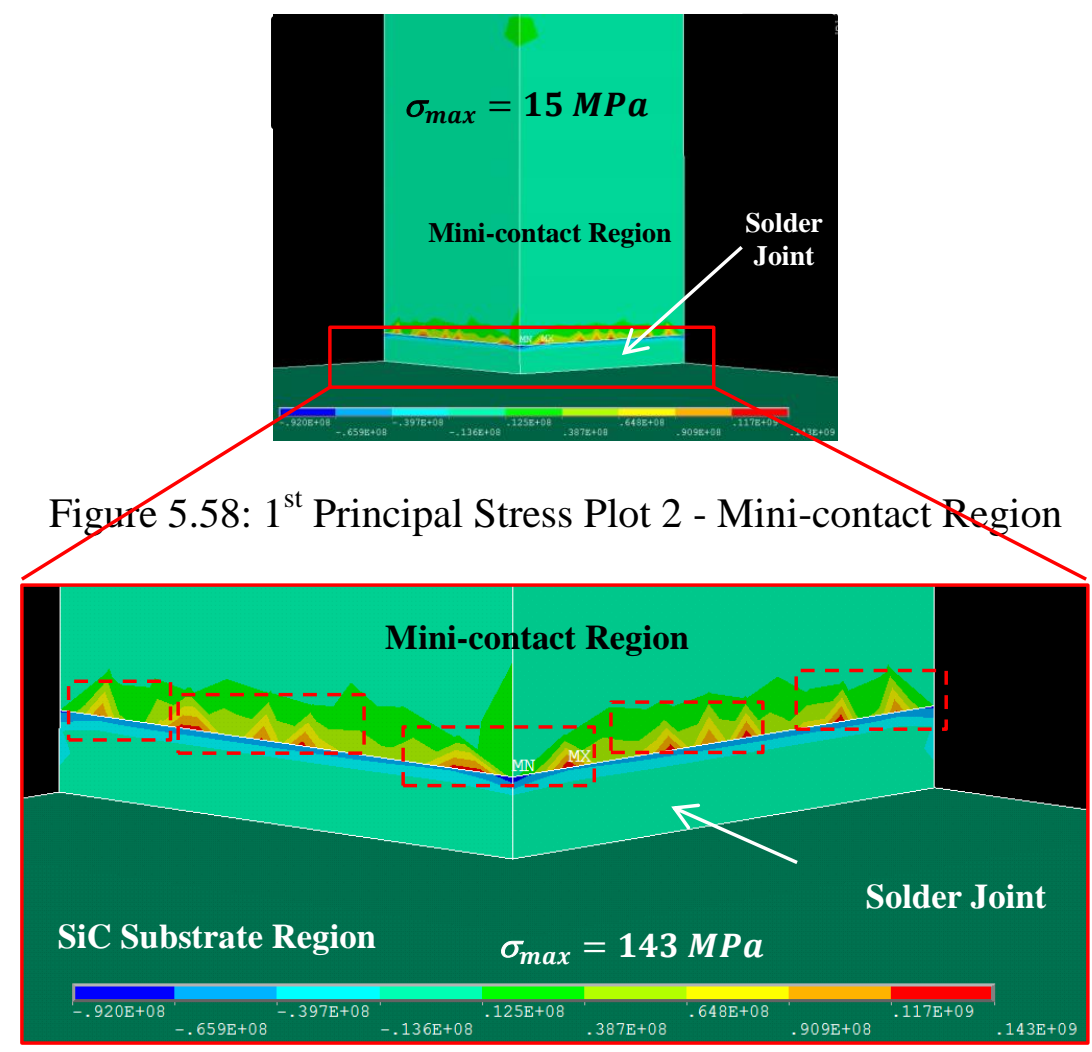

Figure 5.59: $1^{\text {st }}$ Principal Stress Plot 3 - Mini-contact Region 


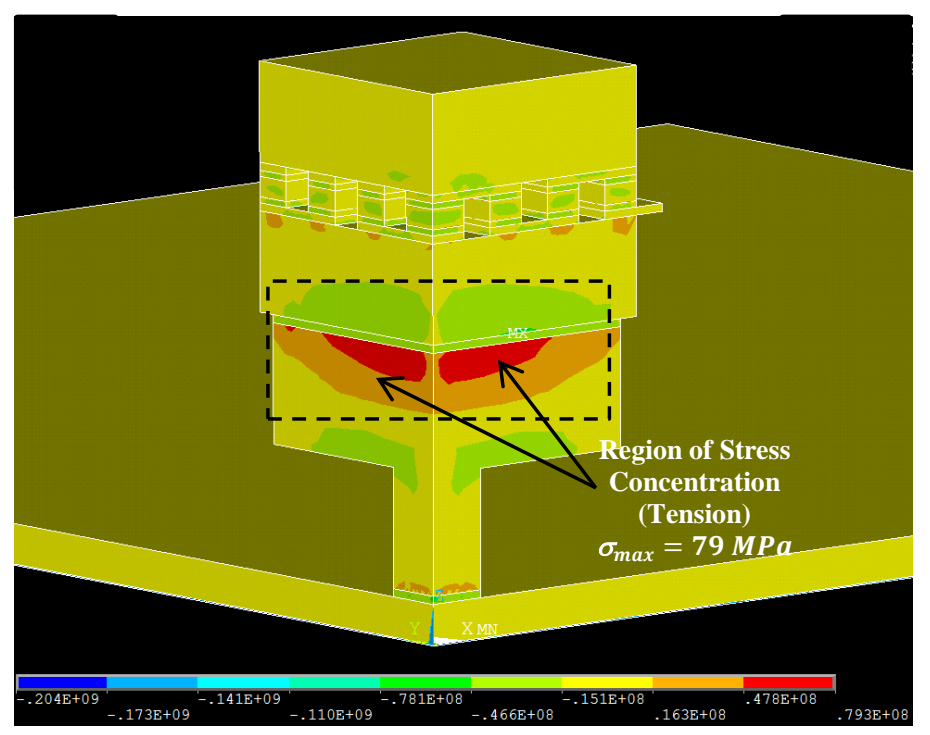

Figure 5.60: $2^{\text {nd }}$ Principal Stress Plot - Diamond Mini-contact Region

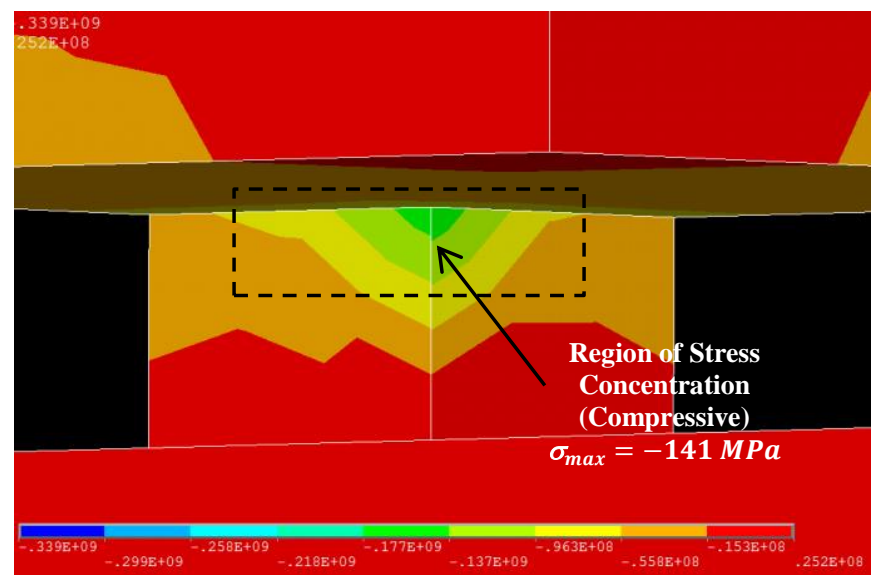

Figure 5.61: $3^{\text {rd }}$ Principal Stress Plot 1 - Diamond Mini-contact Region

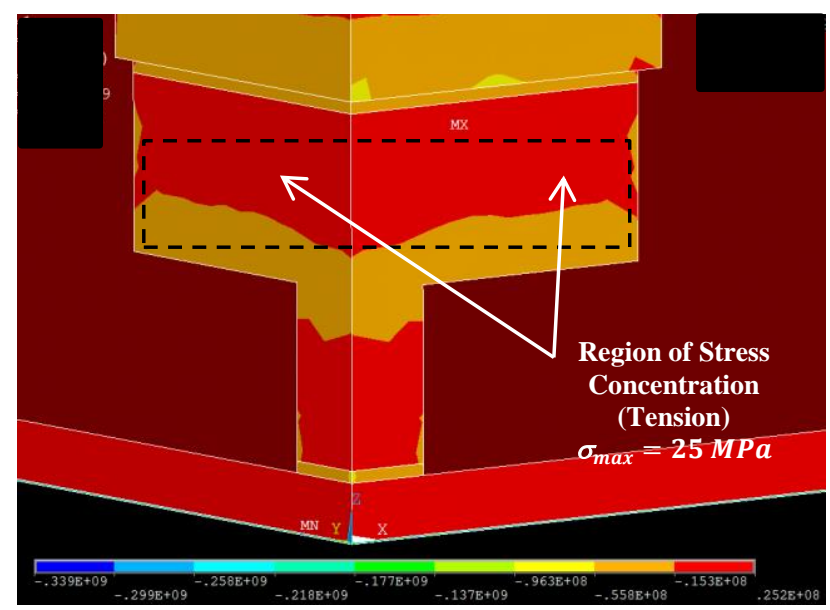

Figure 5.62: $3^{\text {rd }}$ Principal Stress Plot 2 - Mini-contact Region 
From figures 5.57 - 5.62, we can determine a maximum principal stress of 143 MPa in the Mini-contact structure. Using the linear elastic fracture mechanics approach, the critical flaw size can be found out to be $530 \mu \mathrm{m}$ for $\mathrm{K}_{\mathrm{IC}}=7 \mathrm{MPa}^{*} \mathrm{~m}^{1 / 2}$ $[41,42]$ and $Y=1.2$. The flaw size is relatively large considering the dimensions of Mini-contact structure and thus the stresses are under safe limit.

\subsubsection{Critical Flaw Size - Thermo-electric Elements and Headers}

The principal stresses in thermo-electric elements were determined from simulation and are plotted as shown in figures 5.63 - 5.65 below.

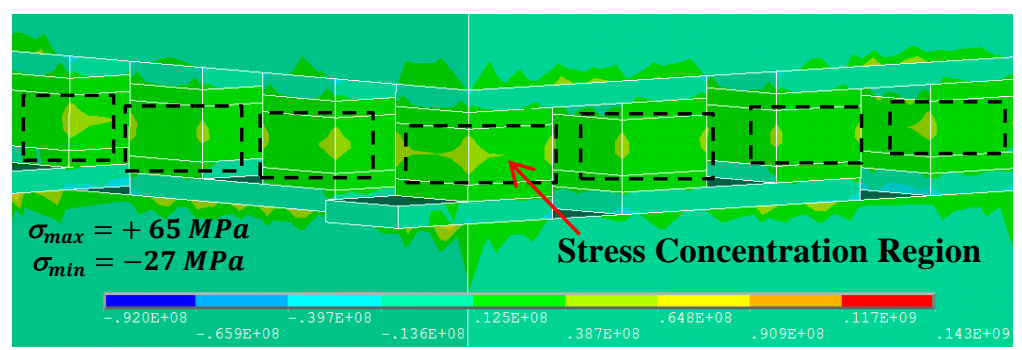

Figure 5.63: $1^{\text {st }}$ Principal Stress Plot - Thermoelectric Elements

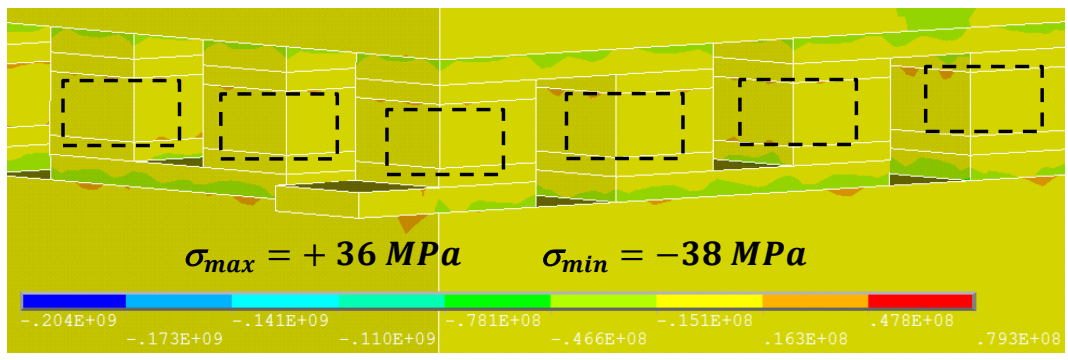

Figure 5.64: $2^{\text {nd }}$ Principal Stress Plot - TE Elements

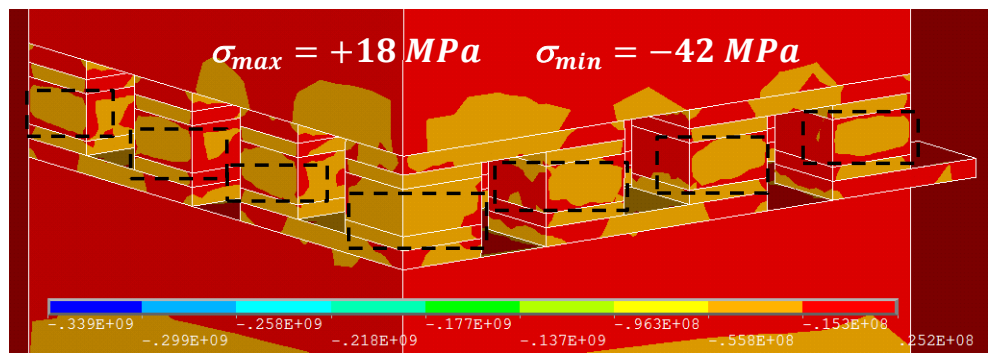

Figure 5.65: $3^{\text {rd }}$ Principal Stress Plot - TE Elements 
Table 5.27: Principal Stresses - Thermoelectric Elements

\begin{tabular}{|c|c|c|c|}
\hline Parameter & $\begin{array}{c}\mathbf{1}^{\text {st }} \text { Principal } \\
\text { Stress }\end{array}$ & $\begin{array}{c}\mathbf{2}^{\text {nd }} \text { Principal } \\
\text { Stress }\end{array}$ & $\begin{array}{c}\mathbf{3}^{\text {rd }} \text { Principal } \\
\text { Stress }\end{array}$ \\
\hline Maximum & $65 \mathrm{MPa}$ & $36 \mathrm{MPa}$ & $18 \mathrm{MPa}$ \\
\hline Node Number & 81806 & 81454 & 74316 \\
\hline Minimum & $-27 \mathrm{MPa}$ & $-38 \mathrm{MPa}$ & $-42 \mathrm{MPa}$ \\
\hline Node Number & 88941 & 89291 & 89278 \\
\hline
\end{tabular}

For a maximum principal stress of $65 \mathrm{MPa}$, the critical flaw size was determined to be in the range of $52 \mu \mathrm{m}-210 \mu \mathrm{m}$ for $\mathrm{Y}=1.2$ and $\mathrm{K}_{\mathrm{IC}}=1 \mathrm{MPa}^{*} \mathrm{~m}^{1 / 2}$ $2 \mathrm{MPa}^{*} \mathrm{~m}^{1 / 2}$. The stresses are under safe limit.

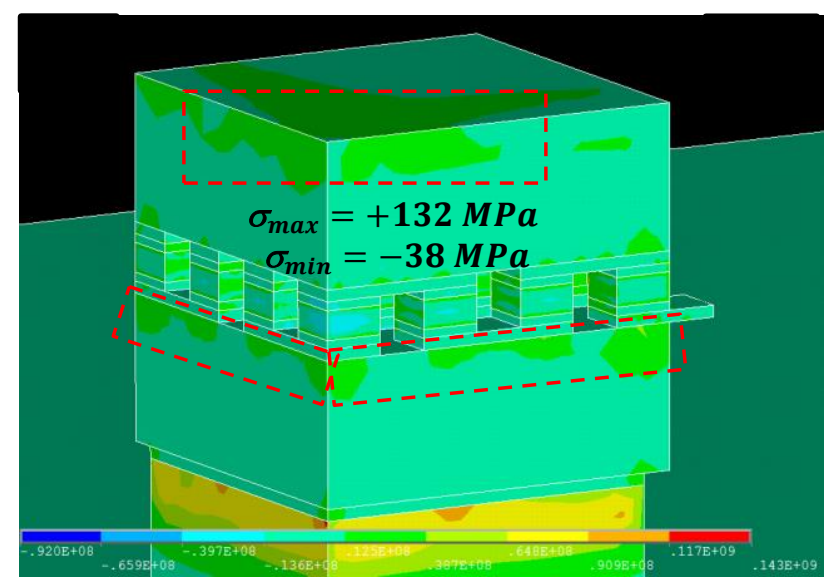

Figure 5.66: $1^{\text {st }}$ Pr. Stress Plot - TE Cooler Headers

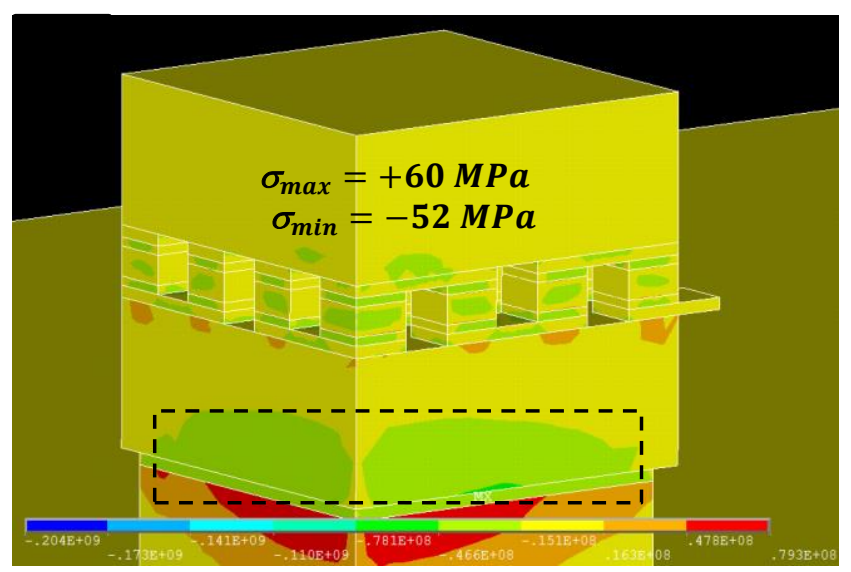

Figure 5.67: $2^{\text {nd }}$ Pr. Stress Plot - TE Cooler Headers 


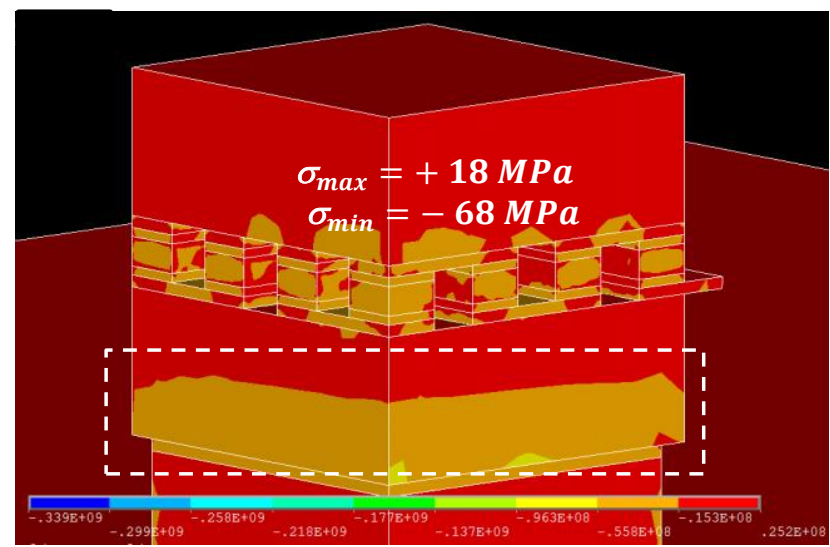

Figure 5.68: $3^{\text {rd }}$ Pr. Stress Plot - TE Cooler Headers

A maximum principal stress of $132 \mathrm{MPa}$ was determined from analysis.

Table 5.28: Principal Stresses - Thermoelectric Cooler Headers

\begin{tabular}{|c|c|c|c|}
\hline Parameter & $\begin{array}{c}\mathbf{1}^{\text {st }} \text { Principal } \\
\text { Stress }\end{array}$ & $\begin{array}{c}\mathbf{2}^{\text {nd }} \text { Principal } \\
\text { Stress }\end{array}$ & $\begin{array}{c}\mathbf{3}^{\text {rd }} \text { Principal } \\
\text { Stress }\end{array}$ \\
\hline Maximum & $\mathbf{1 3 2} \mathrm{MPa}$ & $60 \mathrm{MPa}$ & $18 \mathrm{MPa}$ \\
\hline Node Number & 101719 & 101719 & 126312 \\
\hline Minimum & $-38 \mathrm{MPa}$ & $-52 \mathrm{MPa}$ & $-68 \mathrm{MPa}$ \\
\hline Node Number & 108301 & 108301 & 56120 \\
\hline
\end{tabular}

For a maximum principal stress plot of $132 \mathrm{MPa}$, the critical flaw size was

determined to be $86 \mu \mathrm{m}$ for $\mathrm{Y}=1.2$ and $\mathrm{K}_{\mathrm{IC}}=2.6 \mathrm{MPa}^{*} \mathrm{~m}^{1 / 2}$. Considering the size of the AlN headers, the headers are under safe stress limit.

5.5.6 Critical Flaw Size - GaN on SiC substrate

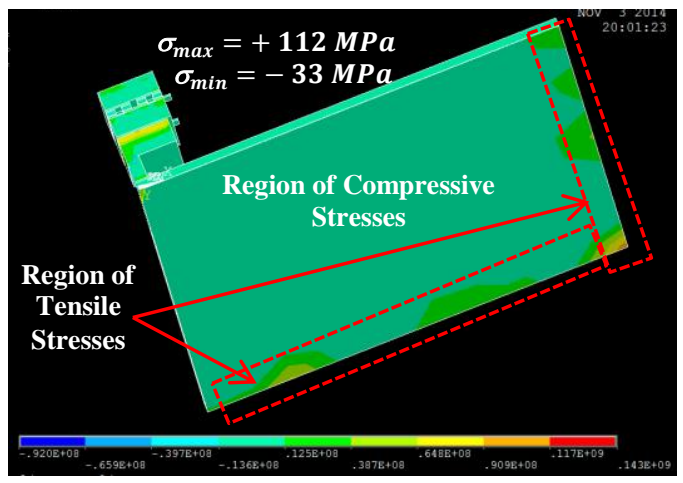

Figure 5.69: $1^{\text {st }}$ Principal Stress Plot - GaN on SiC substrate 


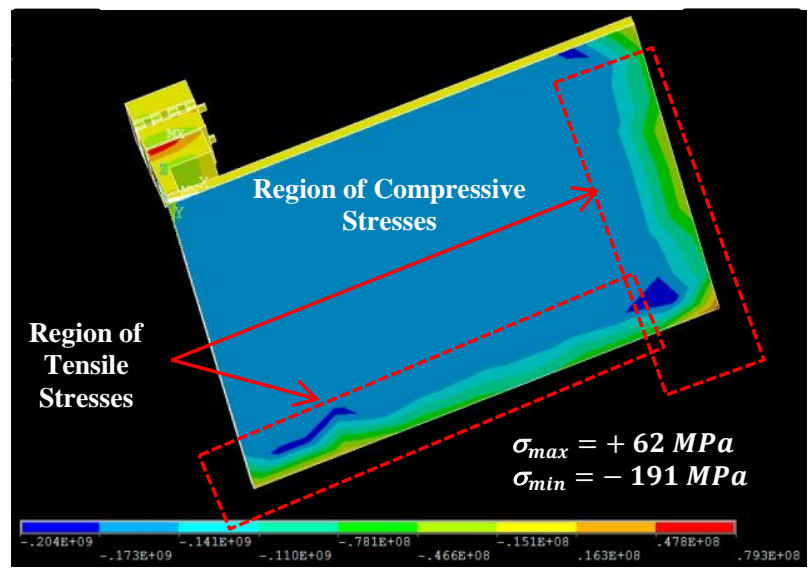

Figure 5.70: $2^{\text {nd }}$ Principal Stress Plot $-\mathrm{GaN}$ on SiC substrate

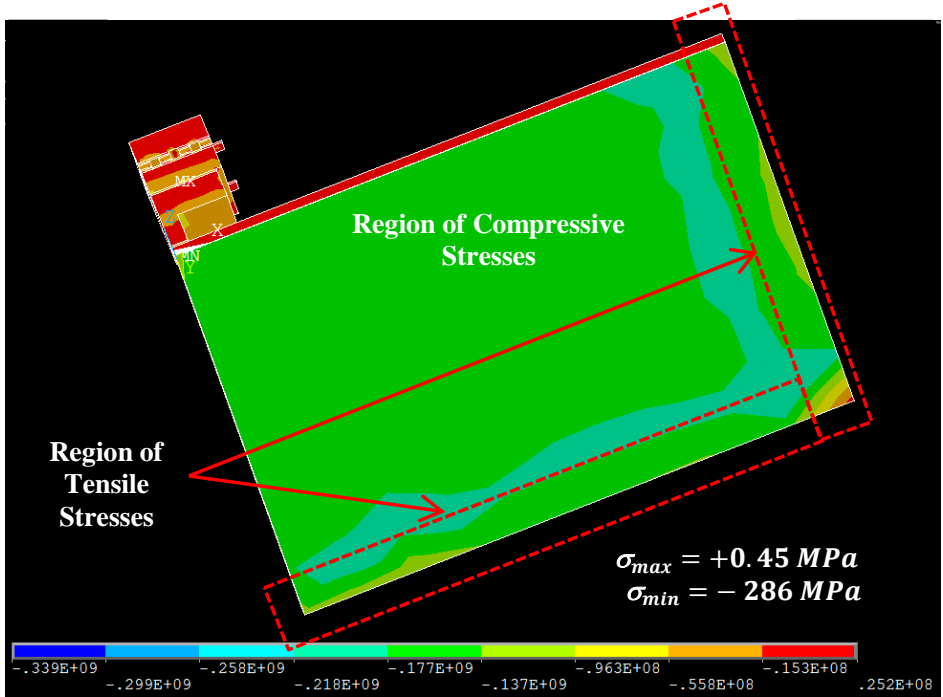

Figure 5.71: $3^{\text {rd }}$ Principal Stress Plot $-\mathrm{GaN}$ on SiC substrate

Table 5.29: Principal Stresses - GaN on SiC substrate

\begin{tabular}{|c|c|c|c|}
\hline Parameter & $\begin{array}{c}\mathbf{1}^{\text {st }} \text { Principal } \\
\text { Stress }\end{array}$ & $\begin{array}{c}\mathbf{2}^{\text {nd }} \text { Principal } \\
\text { Stress }\end{array}$ & $\begin{array}{c}\mathbf{3}^{\text {rd }} \text { Principal } \\
\text { Stress }\end{array}$ \\
\hline Maximum & $\mathbf{1 1 2} \mathbf{M P a}$ & $62 \mathrm{MPa}$ & $0.45 \mathrm{MPa}$ \\
\hline Node Number & 178099 & 178099 & 178099 \\
\hline Minimum & $-33 \mathrm{MPa}$ & $-191 \mathrm{MPa}$ & $-\mathbf{2 8 6} \mathbf{M P a}$ \\
\hline Node Number & 2919 & 178441 & 2919 \\
\hline
\end{tabular}

A critical flaw size of $21 \mu \mathrm{m}$ was calculated based on linear elastic fracture mechanics approach for a maximum principal stress of $112 \mathrm{MPa}, \mathrm{Y}=1.2$ and $\mathrm{K}_{\mathrm{IC}}=$ 1.1 $\mathrm{MPa}^{*} \mathrm{~m}^{1 / 2}$. A negative stress of $-286 \mathrm{MPa}$ was seen at the center of GaN device 
near the hot-spot region. The compressive stresses are supposed to have an adverse effect on device performance by reverse piezoelectric effect.

\subsection{Discrete Mini-contact: Diamond - Lofted Model}

5.6.1 Nodal Temperature Plot

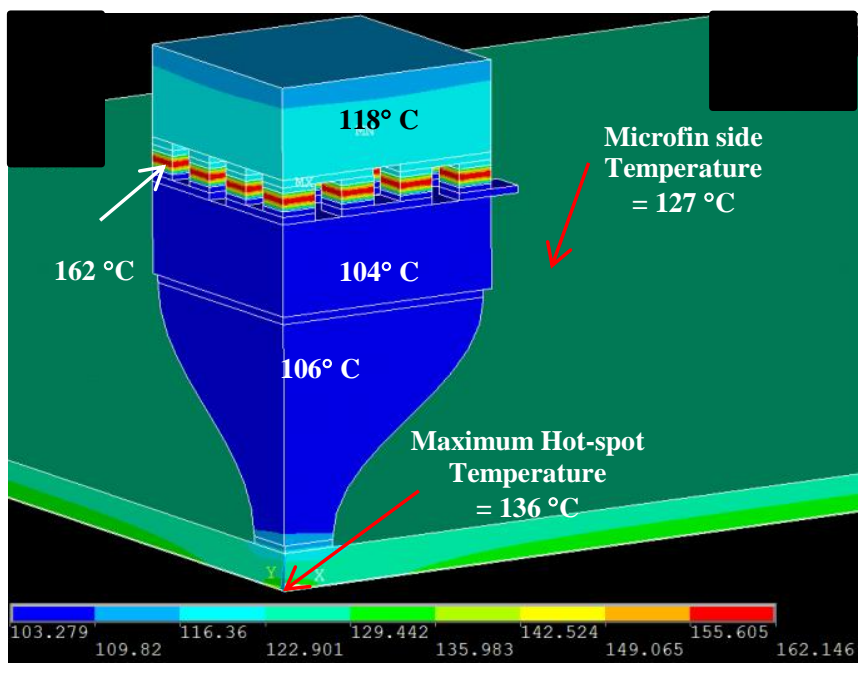

Figure 5.72: Temperature Contour Plot

Figure 5.72 gives temperature across the 3D Mini-contact structure. As it can be inferred from the plot above, a maximum hot-spot temperature of $136{ }^{\circ} \mathrm{C}$ was determined from simulations. The temperature on micro-fin side was found out to be $127{ }^{\circ} \mathrm{C}$ with maximum temperature across thermo-electrics to be about $162{ }^{\circ} \mathrm{C}$. 


\subsubsection{Displacement Contour Plot}

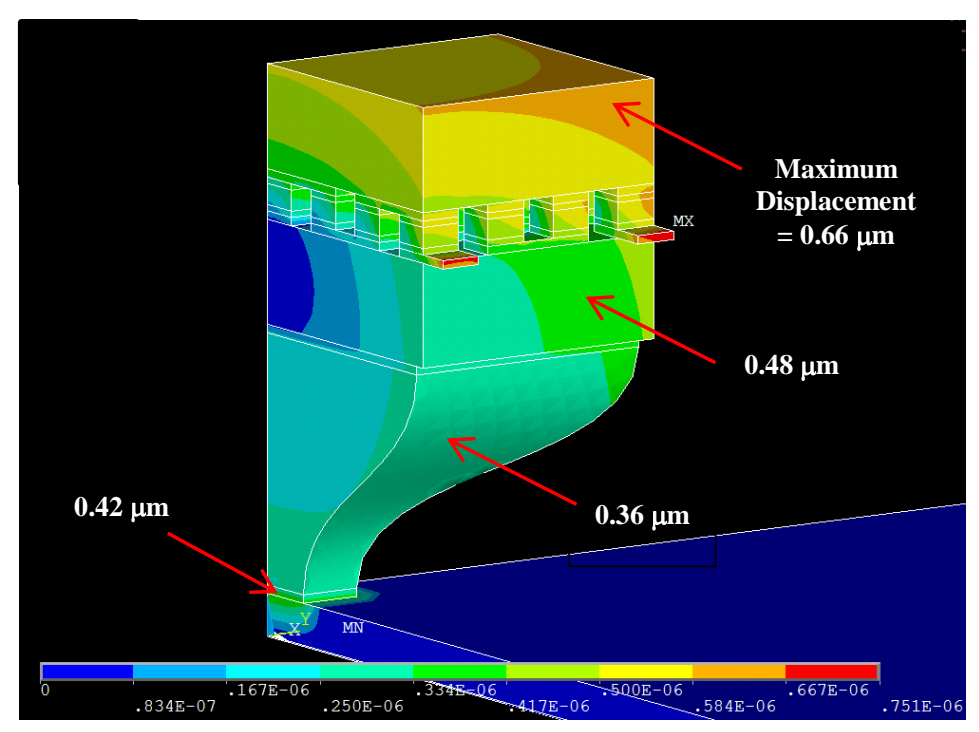

Figure 5.73: Displacement Contour Plot

The maximum deformation across the Mini-contact structure is determined to be at thermo-electric cooler top edge and its magnitude is $0.66 \mu \mathrm{m}$. The deformation across the solder joint interconnecting Mini-contact with SiC chip was found to be around $0.42 \mu \mathrm{m}$.

For chip level integration, $\mathrm{N}_{\mathrm{f}}(50 \%)$ : 478 cycles, 801 cycles and 2,402 cycles. $\mathrm{N}_{\mathrm{f}}(63.2 \%)$ : 540 cycles, 905 cycles and 2,714 cycles. For contact/cooler integration, $\mathrm{N}_{\mathrm{f}}(50 \%): 1,182$ cycles, 2,124 cycles and 7,413 cycles. For integration of $\mathrm{Bi}_{2} \mathrm{Te}_{3}$ elements, $\mathrm{N}_{\mathrm{f}}(50 \%)$ : 2,033 cycles, 3,763 cycles and 13,923 cycles. $\mathrm{N}_{\mathrm{f}}(63.2 \%): 2,296$ cycles, 4,251 cycles and 15,730 cycles. 
5.6.3 Design for Reliability: Mean Time to Failure - MTTF

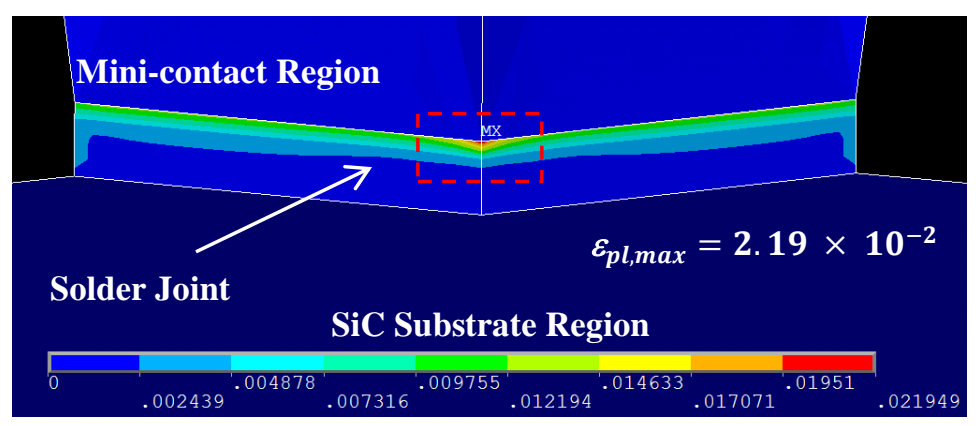

Figure 5.74: Pl. Strain in Solder: Chip Level Integration

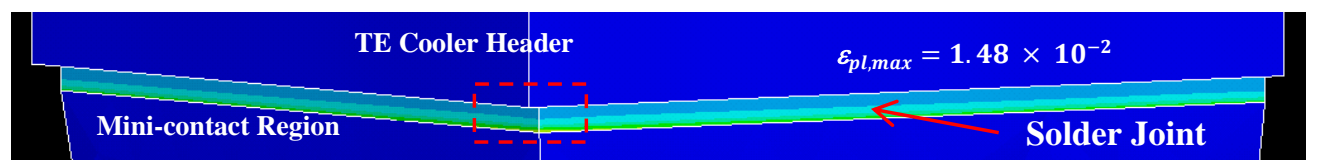

Figure 5.75: Pl. Strain in Solder: Mini-contact/Cooler Integration Level

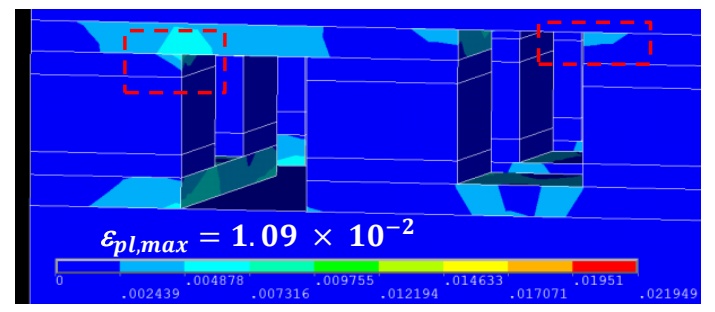

Figure 5.76: Pl. Strain in Solder - TE Cooler

Figures 5.74 - 5.76 are plastic strain in SAC305 solder of magnitude $2.19 \mathrm{x}$ $10^{-2}, 1.48 \times 10^{-2}$ and $1.09 \times 10^{-2}$ determined for chip level, Mini-contact/Cooler level and TE Cooler integration levels respectively. MTTF values are as follows.

Table 5.30: Design for Reliability - Solder MTTF for Chip Level Integration

\begin{tabular}{|c|c|}
\hline Maximum Plastic Strain & $2.19 \mathrm{e}-2$ \\
\hline Maximum Temperature & $110.96^{\circ} \mathrm{C}$ \\
\hline Minimum Temperature & $25^{\circ} \mathrm{C}$ \\
\hline Average Temperature & $67.53{ }^{\circ} \mathrm{C}$ \\
\hline Node Number & 23260 \\
\hline MTTF (596 mins.) & $\mathbf{3 6 8}$ cycles \\
\hline MTTF (20 mins.) & $\mathbf{6 1 7}$ cycles \\
\hline MTTF (1 min.) & $\mathbf{1 , 8 4 9}$ cycles \\
\hline
\end{tabular}


Table 5.31: Design for Reliability - Solder MTTF for Contact/Cooler Integration

\begin{tabular}{|c|c|}
\hline Maximum Plastic Strain & $1.48 \mathrm{e}-2$ \\
\hline Maximum Temperature & $105.90^{\circ} \mathrm{C}$ \\
\hline Minimum Temperature & $25^{\circ} \mathrm{C}$ \\
\hline Average Temperature & $65.45^{\circ} \mathrm{C}$ \\
\hline Node Number & 55655 \\
\hline MTTF (596 mins.) & $\mathbf{1 , 0 5 5}$ cycles \\
\hline MTTF (20 mins.) & $\mathbf{1 , 6 3 5}$ cycles \\
\hline MTTF (1 min.) & $\mathbf{5 , 7 0 5}$ cycles \\
\hline
\end{tabular}

Table 5.32: Design for Reliability - Solder MTTF in TE Cooler

\begin{tabular}{|c|c|}
\hline Maximum Plastic Strain & $1.09 \mathrm{e}-2$ \\
\hline Maximum Temperature & $122.19^{\circ} \mathrm{C}$ \\
\hline Minimum Temperature & $25^{\circ} \mathrm{C}$ \\
\hline Average Temperature & $73.59{ }^{\circ} \mathrm{C}$ \\
\hline Node Number & 114999 \\
\hline MTTF (596 mins.) & $\mathbf{1 , 8 1 5}$ cycles \\
\hline MTTF (20 mins.) & $\mathbf{3 , 3 6 0}$ cycles \\
\hline MTTF (1 min.) & $\mathbf{1 2 , 4 3 3}$ cycles \\
\hline
\end{tabular}

From tables 5.29-5.31, it is shown that the failure site is at the solder joint interconnecting Mini-contact with SiC chip and a MTTF of 368 cycles, 617 cycles and 1,849 cycles for different dwell times was determined based on Engelmaier's model. The solder joints at Mini-contact/Cooler integration were found to have MTTF of 1,055 cycles, 1,635 cycles and 5,705 cycles. The MTTF for solder joint inside the TE cooler was determined to be 1,815 cycles, 3,360 cycles and 12,433 cycles. 
5.6.4 Critical Flaw Size - Mini-contact and Thermo-electric Cooler

Principal stresses across Mini-contact structure are shown as follows.

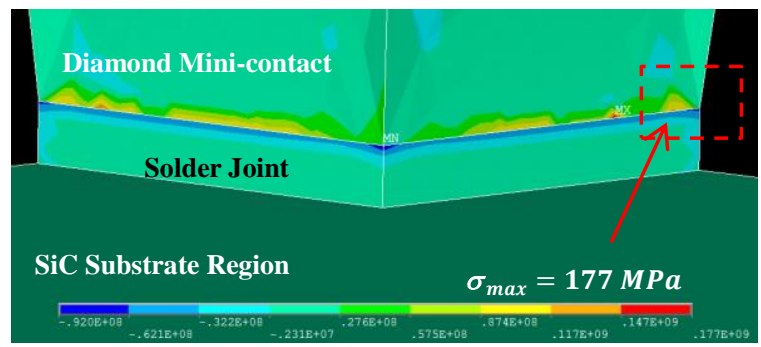

Figure 5.77: $1^{\text {st }}$ Principal Stress Plot 1 - Mini-contact Region

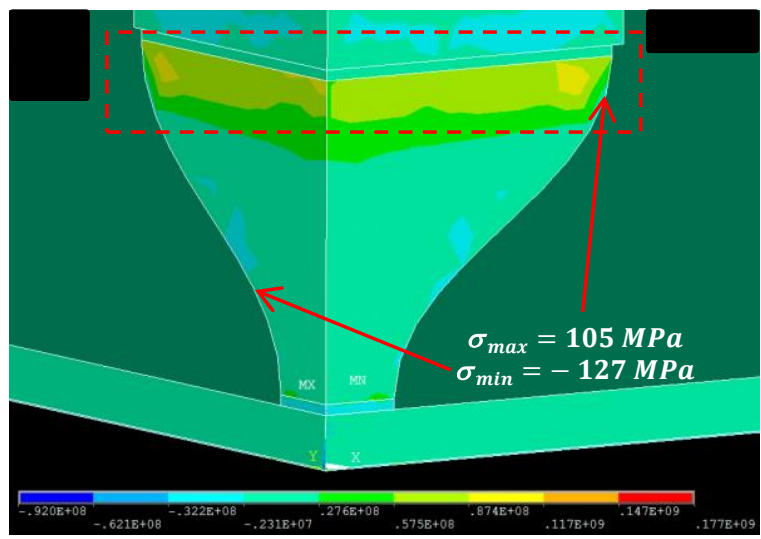

Figure 5.78: $1^{\text {st }}$ Principal Stress Plot 2 - Mini-contact Region

Figures 5.77 and 5.78 are maximum principal stress plots across the Minicontact structure determined from thermo-mechanical simulations. The second and third principal stresses are compressive in nature and thus, will not contribute to brittle fracture mechanism. For a maximum principal stress of $177 \mathrm{MPa}$, the critical flaw size was determined to be $346 \mu \mathrm{m}$ for $\mathrm{Y}=1.2$ and $\mathrm{K}_{\mathrm{IC}}=7 \mathrm{MPa}^{*} \mathrm{~m}^{1 / 2}$.

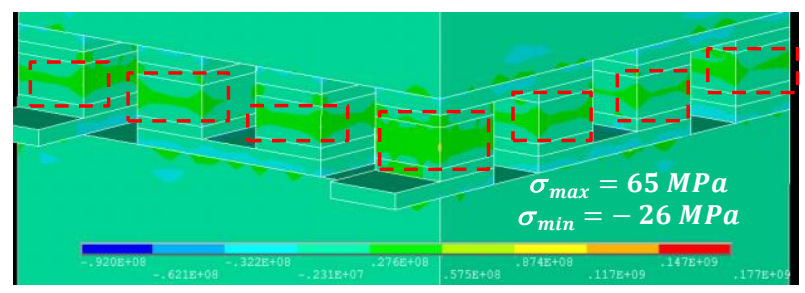

Figure 5.79: $1^{\text {st }}$ Principal Stress Plot - Thermoelectric Elements 


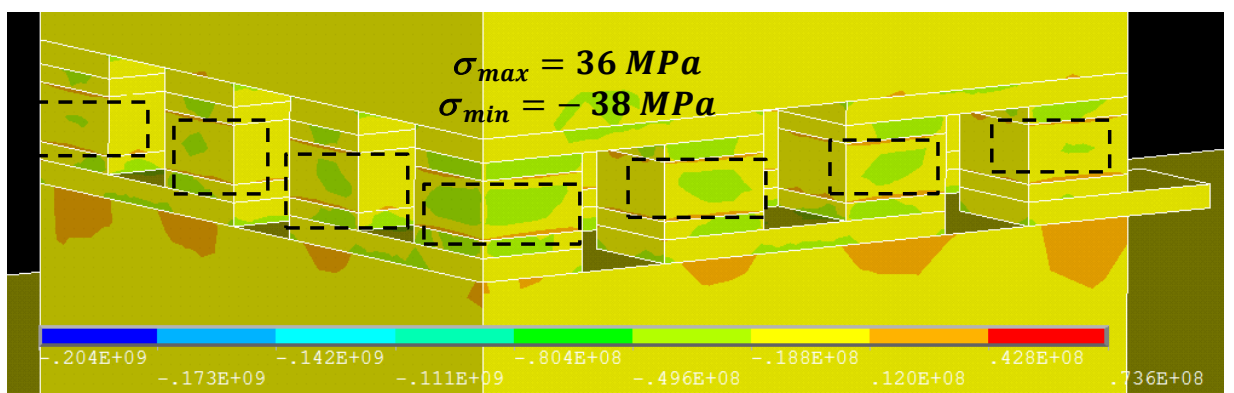

Figure 5.80: $2^{\text {nd }}$ Principal Stress Plot - Thermoelectric Elements

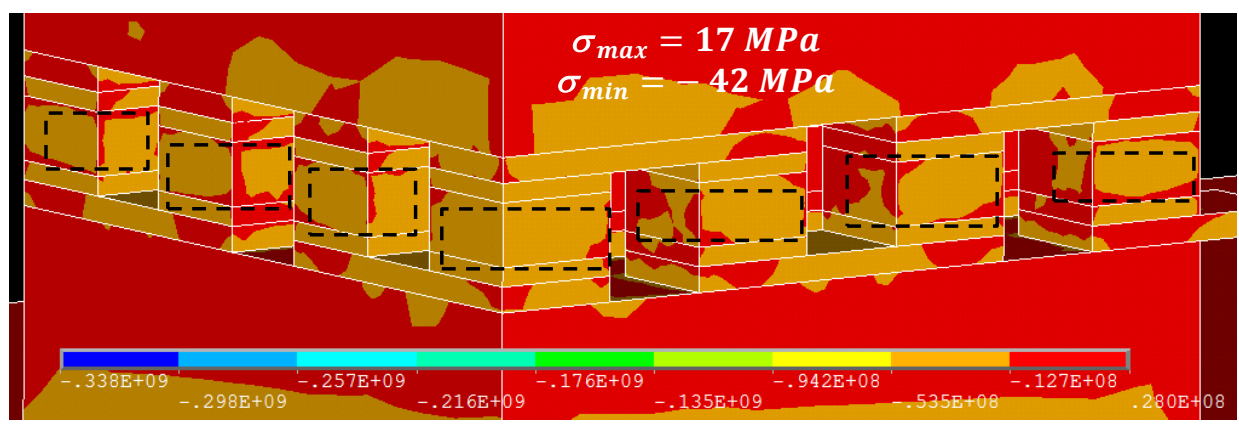

Figure 5.81: $3^{\text {rd }}$ Principal Stress Plot - Thermoelectric Elements

Table 5.33: Principal Stresses - Thermoelectric Elements

\begin{tabular}{|c|c|c|c|}
\hline Parameter & $\begin{array}{c}\mathbf{1}^{\text {st }} \text { Principal } \\
\text { Stress }\end{array}$ & $\begin{array}{c}\mathbf{2}^{\text {nd }} \text { Principal } \\
\text { Stress }\end{array}$ & $\begin{array}{c}\mathbf{3}^{\text {rd }} \text { Principal } \\
\text { Stress }\end{array}$ \\
\hline Maximum & $65 \mathrm{MPa}$ & $36 \mathrm{MPa}$ & $17 \mathrm{MPa}$ \\
\hline Node Number & 81806 & 83840 & 74316 \\
\hline Minimum & $-26 \mathrm{MPa}$ & $-38 \mathrm{MPa}$ & $-42 \mathrm{MPa}$ \\
\hline Node Number & 88941 & 89291 & 89278 \\
\hline
\end{tabular}

A maximum principal stress of $65 \mathrm{MPa}$ was determined at the corners of

thermo-electric elements from thermo-mechanical simulations. Based on linear elastic fracture mechanics approach, a critical flaw size in the range of $52 \mu \mathrm{m}-210 \mu \mathrm{m}$ was found out for a $\mathrm{Y}=1.2$ and $\mathrm{K}_{\mathrm{IC}}$ in range of $1 \mathrm{MPa}^{*} \mathrm{~m}^{1 / 2}-2 \mathrm{MPa}^{*} \mathrm{~m}^{1 / 2}$. 


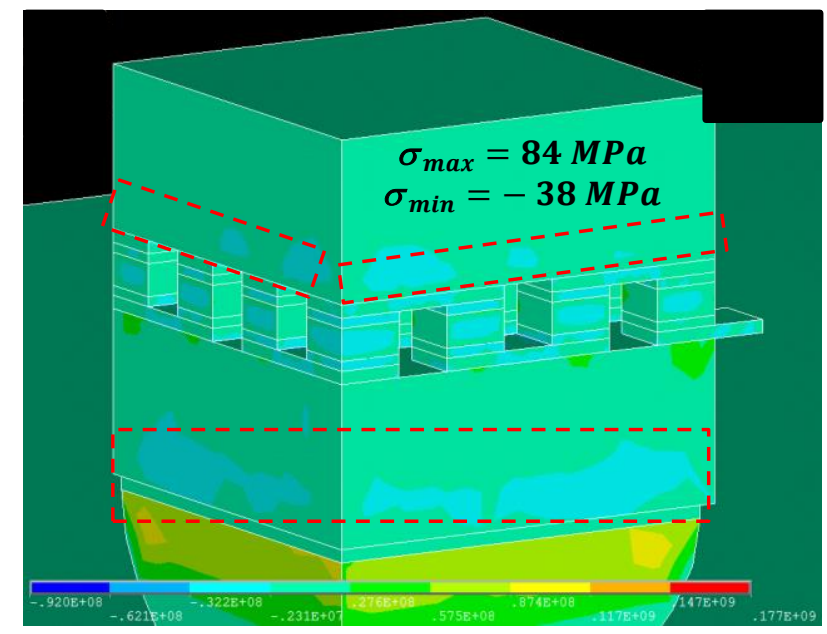

Figure 5.82: $1^{\text {st }}$ Principal Stress Plot - Thermoelectric Cooler Header

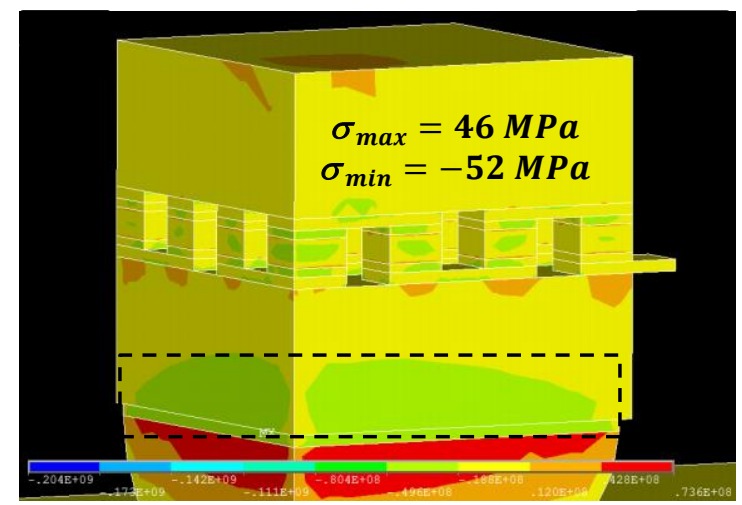

Figure 5.83: $2^{\text {nd }}$ Principal Stress Plot - Thermoelectric Cooler Header

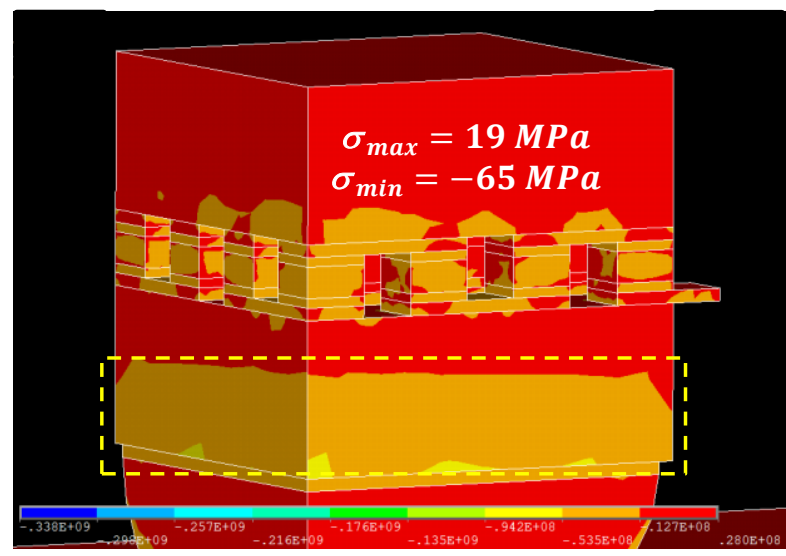

Figure 5.84: $3^{\text {rd }}$ Principal Stress Plot - Thermoelectric Cooler Header 
Table 5.34: Principal Stresses - Thermoelectric Headers

\begin{tabular}{|c|c|c|c|}
\hline Parameter & $\begin{array}{c}\mathbf{1}^{\text {st }} \text { Principal } \\
\text { Stress }\end{array}$ & $\begin{array}{c}\mathbf{2}^{\text {nd }} \text { Principal } \\
\text { Stress }\end{array}$ & $\begin{array}{c}\mathbf{3}^{\text {rd }} \text { Principal } \\
\text { Stress }\end{array}$ \\
\hline Maximum & $84 \mathrm{MPa}$ & $46 \mathrm{MPa}$ & $19 \mathrm{MPa}$ \\
\hline Node Number & 101719 & 101721 & 101847 \\
\hline Minimum & $-38 \mathrm{MPa}$ & $-52 \mathrm{MPa}$ & $-65 \mathrm{MPa}$ \\
\hline Node Number & 108301 & 108301 & 55972 \\
\hline
\end{tabular}

A maximum principal stress of $84 \mathrm{MPa}$ was determined through thermo-

mechanical simulations. The critical flaw size for this stress level was determined to be $212 \mu \mathrm{m}$ for a $\mathrm{Y}=1.2$ and $\mathrm{K}_{\mathrm{IC}}$ of $2.6 \mathrm{MPa}^{*} \mathrm{~m}^{1 / 2}$. The flaw size is relatively large and will not be of a concern. Thus, stresses are under safe limit.

5.6.5 Critical Flaw Size - GaN on $\mathrm{SiC}$ substrate

Table 5.35: Principal Stresses - GaN on SiC substrate

\begin{tabular}{|c|c|c|c|}
\hline Parameter & $\begin{array}{c}\mathbf{1}^{\text {st }} \text { Principal } \\
\text { Stress }\end{array}$ & $\begin{array}{c}\mathbf{2}^{\text {nd }} \text { Principal } \\
\text { Stress }\end{array}$ & $\begin{array}{c}\mathbf{3}^{\text {rd }} \text { Principal } \\
\text { Stress }\end{array}$ \\
\hline Maximum & $\mathbf{1 1 2} \mathbf{M P a}$ & $62 \mathrm{MPa}$ & $0.45 \mathrm{MPa}$ \\
\hline Node Number & 185126 & 185126 & 185126 \\
\hline Minimum & $-33 \mathrm{MPa}$ & $-191 \mathrm{MPa}$ & $\mathbf{- 2 8 5} \mathbf{M P a}$ \\
\hline Node Number & 2919 & 185468 & 2919 \\
\hline
\end{tabular}

$\sigma_{\max }=112 \mathrm{MPa}$ and $\sigma_{\min }=-285 \mathrm{MPa}$

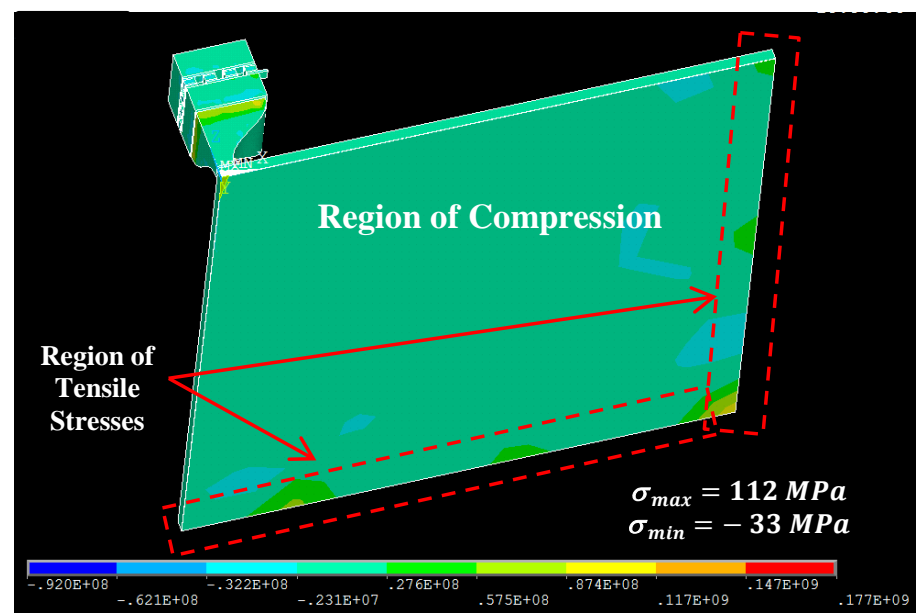

Figure 5.85: $1^{\text {st }}$ Pr. Stress Plot - GaN on SiC substrate 


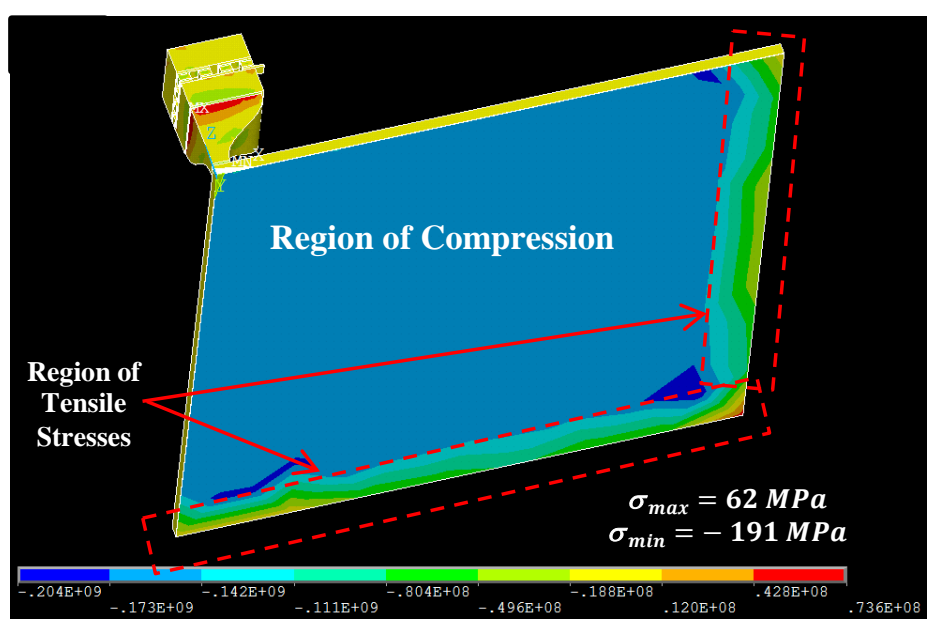

Figure 5.86: $2^{\text {nd }}$ Principal Stress Plot - GaN on SiC substrate

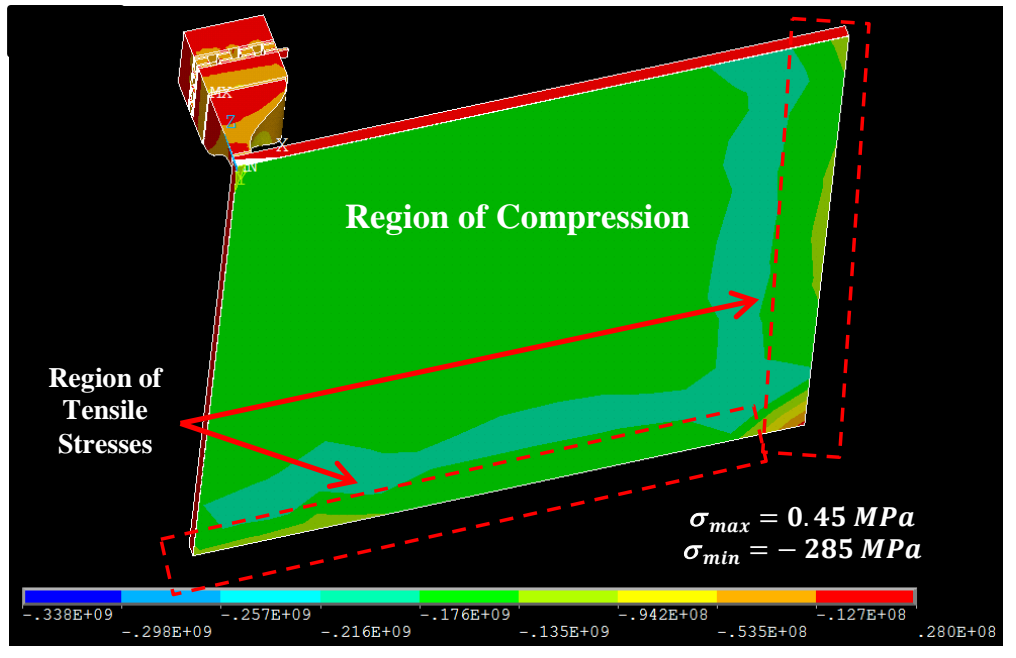

Figure 5.87: $3^{\text {rd }}$ Principal Stress Plot - GaN on SiC substrate 


\subsection{Discrete Mini-contact: Diamond - Taper Model}

5.7.1 Temperature Contour Plot

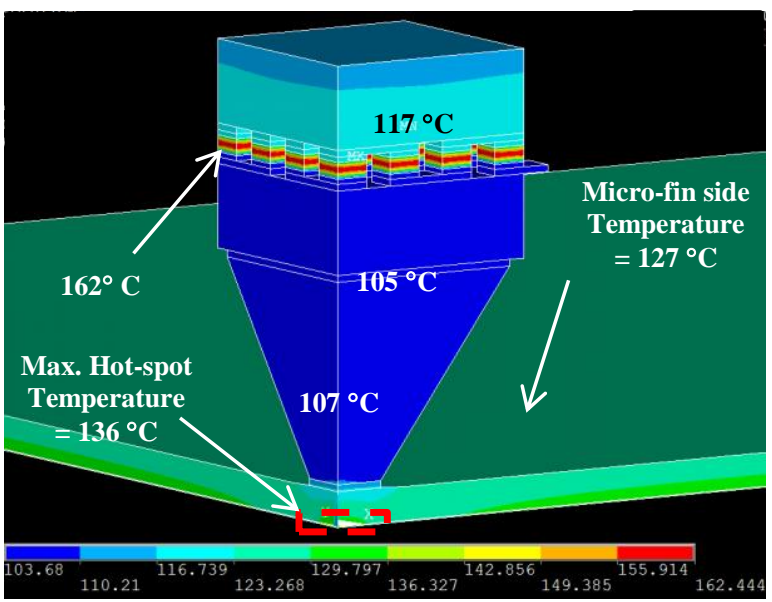

Figure 5.88: Temperature Contour Plot

A maximum hot-spot temperature of $136{ }^{\circ} \mathrm{C}$ is seen at the hot-spot. Micro-fin side temperature is found to be $127^{\circ} \mathrm{C}$ and the hottest part of the device is thermoelectric cooler, which has a temperature of $162{ }^{\circ} \mathrm{C}$.

\subsubsection{Displacement Contour Plot}

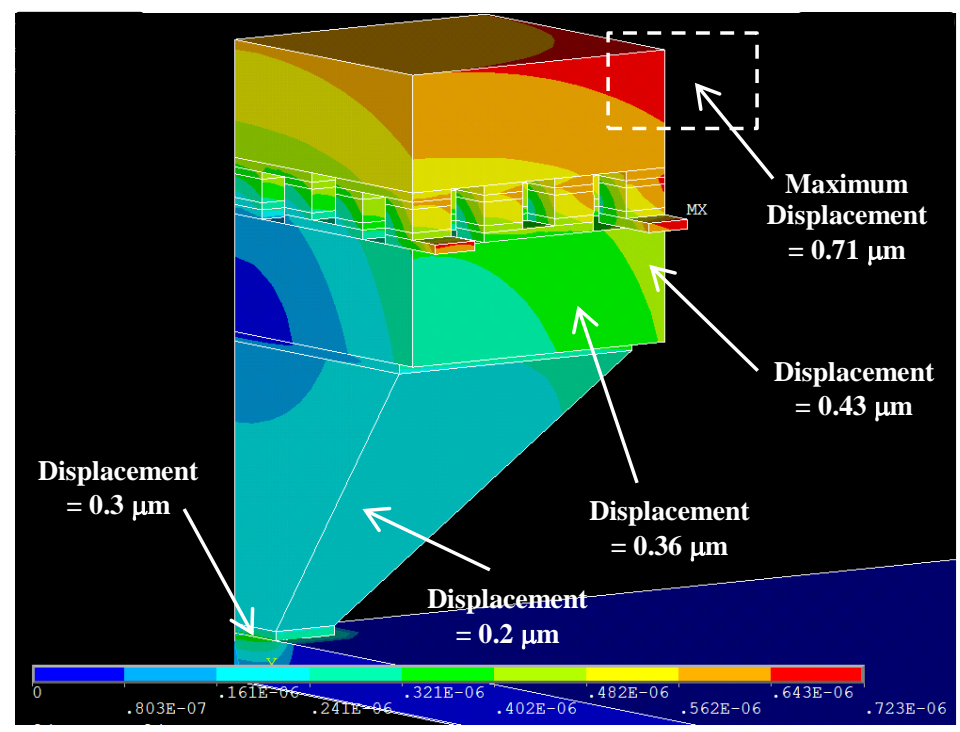

Figure 5.89: Displacement Contour Plot 
A maximum displacement of $0.71 \mu \mathrm{m}$ is seen at the edge of thermo-electric cooler. As mentioned earlier, diamond is a linear elastic material with low C.T.E., resulting in maximum deformation at the thermo-electric cooler edge.

\subsubsection{Design for Reliability: Mean Time to Failure - MTTF}

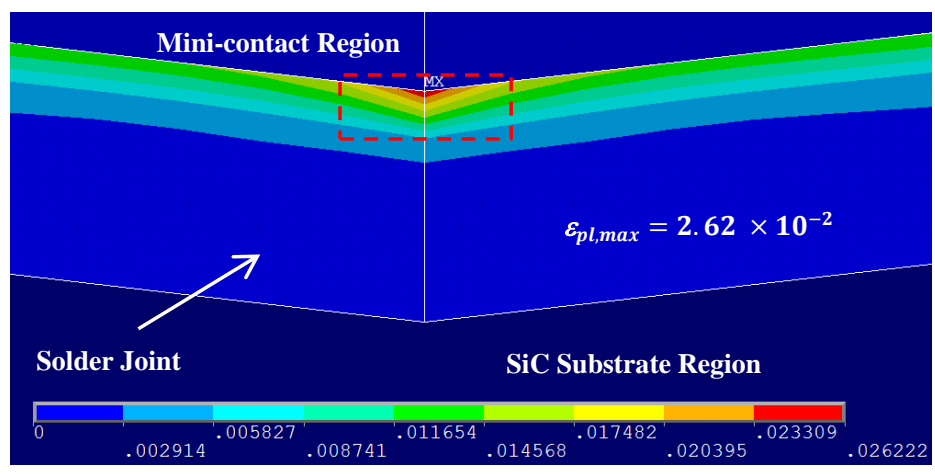

Figure 5.90: Pl. Strain - Solder Joint - Chip Level Integration

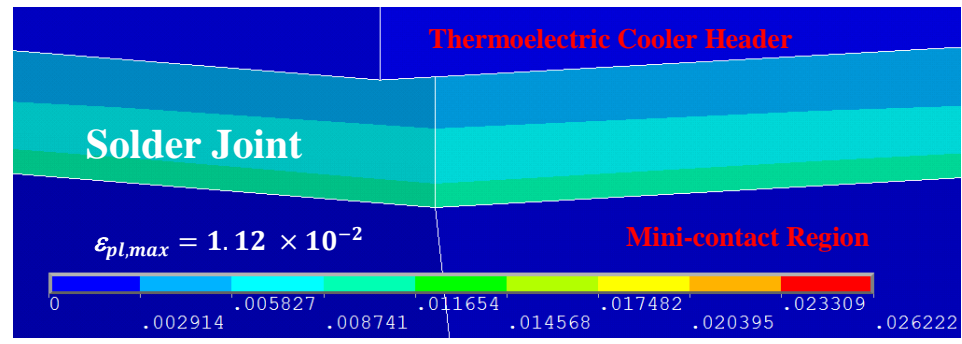

Figure 5.91: Pl. Strain - Solder Joint - Cooler/Mini-contact Integration

Plastic strains of $2.62 \times 10^{-2}$ and $1.12 \times 10^{-2}$ were determined respectively for solder joint at chip level and contact/cooler integration levels. The MTTF of solder joint at these integration levels was determined based on Engelmaier's failure model and presented as shown in following tables.

Chip level integration, $\mathrm{N}_{\mathrm{f}}(50 \%)$ : 323 cycles, 524 cycles and 1,478 cycles. $\mathrm{N}_{\mathrm{f}}$ (63.2\%): 364 cycles, 592 cycles and 1,670 cycles. For contact/cooler integration, $\mathrm{N}_{\mathrm{f}}$ (50\%): 2192 cycles, 4,125 cycles and 15,887 cycles. $\mathrm{N}_{\mathrm{f}}(63.2 \%): 2,476$ cycles, 4,660 
cycles and 17,949 cycles. Inside TE cooler, $\mathrm{N}_{\mathrm{f}}(50 \%)$ : 1,992 cycles, 3,682 cycles and 13,581 cycles. $\mathrm{N}_{\mathrm{f}}(63.2 \%): 2,251$ cycles, 4,160 cycles and 15,344 cycles.

Table 5.36: Design for Reliability - Solder Joint Chip Level Integration

\begin{tabular}{|c|c|}
\hline Maximum Plastic Strain & $2.62 \mathrm{e}-2$ \\
\hline Maximum Temperature & $109.41^{\circ} \mathrm{C}$ \\
\hline Minimum Temperature & $25^{\circ} \mathrm{C}$ \\
\hline Average Temperature & $67.21^{\circ} \mathrm{C}$ \\
\hline Node Number & 14324 \\
\hline MTTF $(\mathbf{5 9 6}$ mins.) & $\mathbf{2 8 8}$ cycles \\
\hline MTTF (20 mins.) & $\mathbf{4 6 7}$ cycles \\
\hline MTTF (1 min.) & $\mathbf{1 , 3 2 0}$ cycles \\
\hline
\end{tabular}

Table 5.37: Design for Reliability - Solder Joint Contact/Cooler Level Integration

\begin{tabular}{|c|c|}
\hline Maximum Plastic Strain & $1.12 \mathrm{e}-2$ \\
\hline Maximum Temperature & $106.43{ }^{\circ} \mathrm{C}$ \\
\hline Minimum Temperature & $25^{\circ} \mathrm{C}$ \\
\hline Average Temperature & $65.72^{\circ} \mathrm{C}$ \\
\hline Node Number & 39753 \\
\hline MTTF (596 mins.) & 1,957 cycles \\
\hline MTTF (20 mins.) & 3,684 cycles \\
\hline MTTF (1 min.) & 14,187 cycles \\
\hline
\end{tabular}

Table 5.38: Design for Reliability - Solder Joint TE Cooler

\begin{tabular}{|c|c|}
\hline Maximum Plastic Strain & $1.10 \mathrm{e}-2$ \\
\hline Maximum Temperature & $122.20^{\circ} \mathrm{C}$ \\
\hline Minimum Temperature & $25^{\circ} \mathrm{C}$ \\
\hline Average Temperature & $73.60^{\circ} \mathrm{C}$ \\
\hline Node Number & 103282 \\
\hline MTTF $(\mathbf{5 9 6}$ mins.) & $\mathbf{1 , 7 7 9}$ cycles \\
\hline MTTF $(\mathbf{2 0}$ mins.) & $\mathbf{3 , 2 8 7}$ cycles \\
\hline MTTF (1 min.) & $\mathbf{1 2 , 1 2 8}$ cycles \\
\hline
\end{tabular}

As seen for other cases of diamond, the solder joint at chip level integration fails first in the electronics package. A MTTF of 288 cycles, 467 cycles and 1,320 cycles was determined for this case. This was followed by MTTF of 1,957 cycles, 
3,684 cycles and 14,187 cycles for Mini-contact/cooler integration. A MTTF of 1,779 cycles, 3,287 cycles and 12,128 cycles was found for solder joint inside the cooler.

\subsubsection{Critical Flaw Size - Mini-contact and TE Cooler}

The stress distribution across a taper Mini-contact (diamond) is shown below in figures $5.92-5.95$.

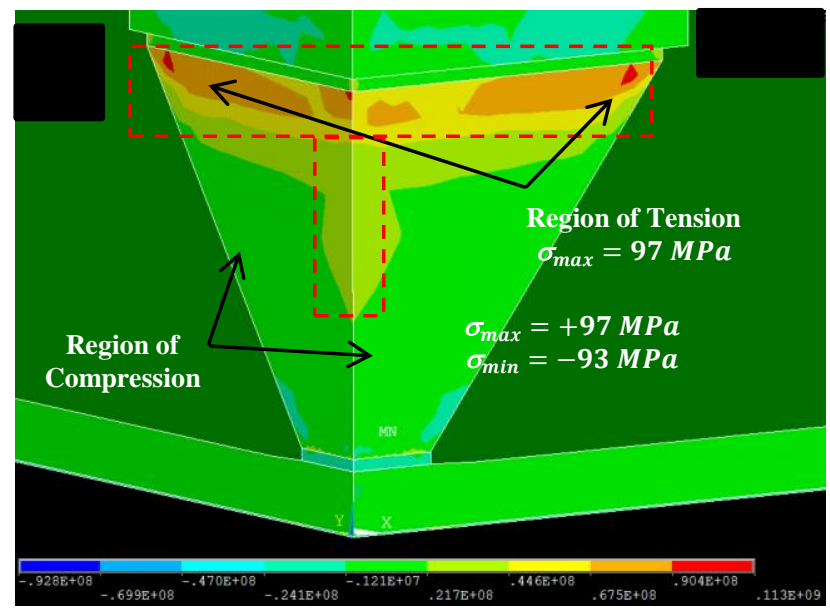

Figure 5.92: $1^{\text {st }}$ Principal Stress Plot 1 - Mini-contact Region

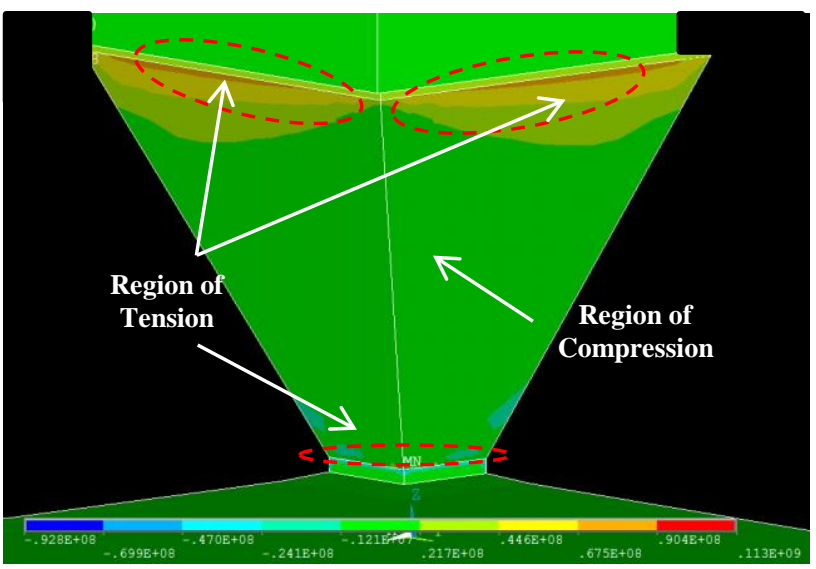

Figure 5.93: $1^{\text {st }}$ Principal Stress Plot 2 - Mini-contact Region 


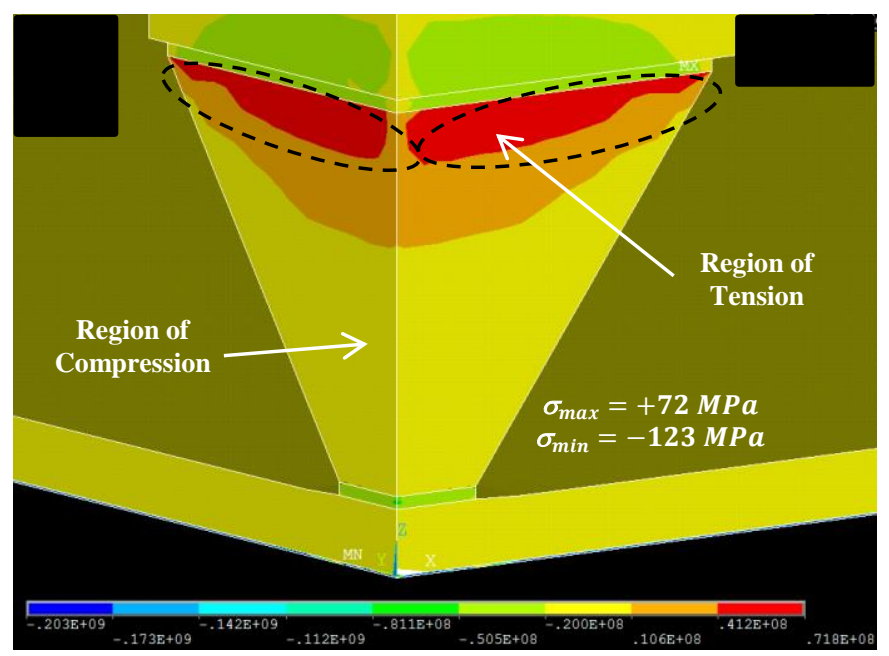

Figure 5.94: $2^{\text {nd }}$ Principal Stress Plot - Mini-contact Region

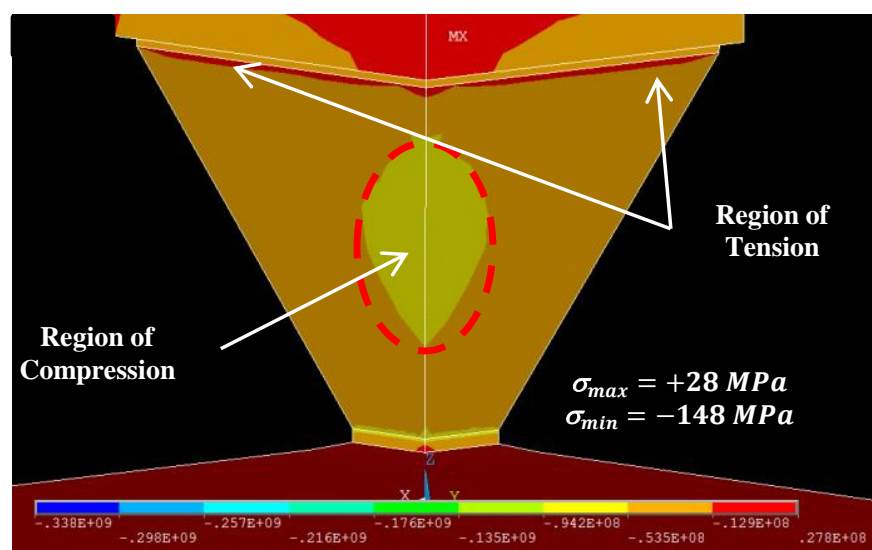

Figure 5.95: $3^{\text {rd }}$ Principal Stress Plot - Mini-contact Region

A critical flaw size of $1.15 \mathrm{~mm}$ was determined for a maximum principal stress of $97 \mathrm{MPa}, \mathrm{Y}=1.2$ and $\mathrm{K}_{\mathrm{IC}}=7 \mathrm{MPa}^{*} \mathrm{~m}^{1 / 2}$. Considering the size of Mini-contact and package, the critical flaw size is relatively large and one can conclude that the structure will not fail under these stresses.

The principal stress distribution across thermo-electric elements is shown in figures $5.96-5.98$. 


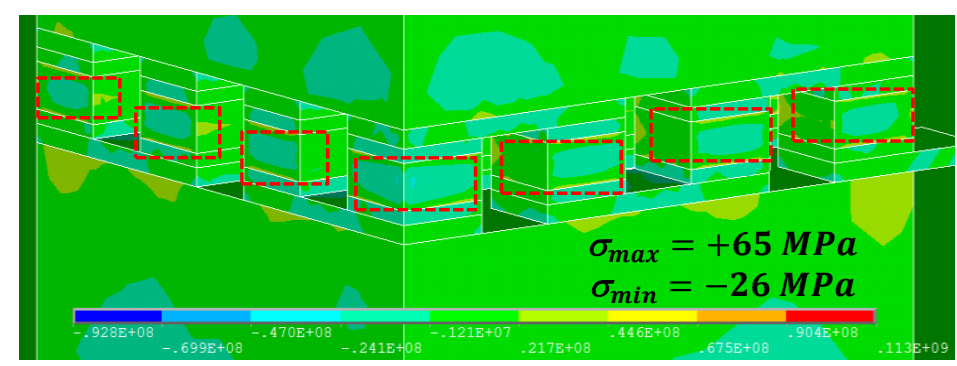

Figure 5.96: $1^{\text {st }}$ Principal Stress Plot - TE Elements

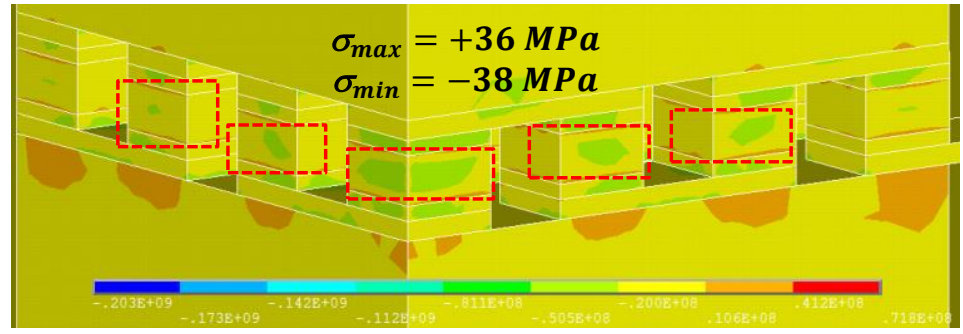

Figure 5.97: $2^{\text {nd }}$ Principal Stress Plot - TE Elements

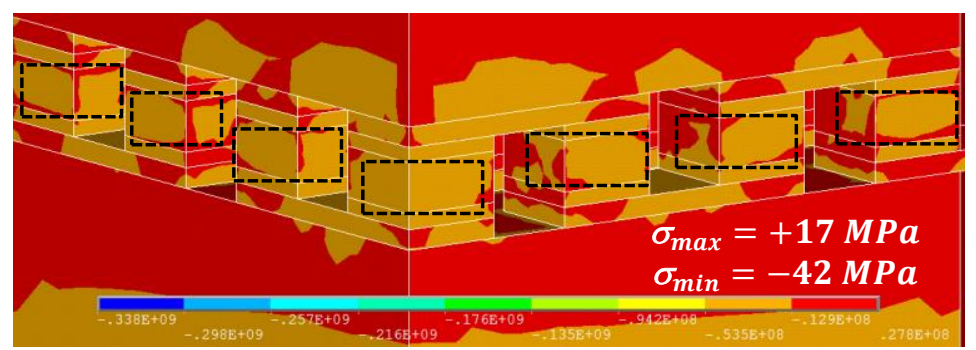

Figure 5.98: $3^{\text {rd }}$ Principal Stress Plot - TE Elements

Table 5.39: Principal Stresses - Thermoelectric Elements

\begin{tabular}{|c|c|c|c|}
\hline Parameter & $\begin{array}{c}\mathbf{1}^{\text {st }} \text { Principal } \\
\text { Stress }\end{array}$ & $\begin{array}{c}\mathbf{2}^{\text {nd }} \text { Principal } \\
\text { Stress }\end{array}$ & $\begin{array}{c}\mathbf{3}^{\text {rd }} \text { Principal } \\
\text { Stress }\end{array}$ \\
\hline Maximum & $\mathbf{6 5} \mathrm{MPa}$ & $36 \mathrm{MPa}$ & $17 \mathrm{MPa}$ \\
\hline Node Number & 90480 & 92514 & 82990 \\
\hline Minimum & $-26 \mathrm{MPa}$ & $-38 \mathrm{MPa}$ & $-42 \mathrm{MPa}$ \\
\hline Node Number & 97615 & 97965 & 97952 \\
\hline
\end{tabular}

A maximum principal stress of $65 \mathrm{MPa}$ was determined from thermo-

mechanical stress analysis. Critical flaw size $=52 \mu \mathrm{m}-210 \mu \mathrm{m}$ for $\mathrm{K}_{\mathrm{IC}}$ in range of 1 $\mathrm{MPa}^{*} \mathrm{~m}^{1 / 2}-2 \mathrm{MPa}^{*} \mathrm{~m}^{1 / 2}$ and $\mathrm{Y}=1.2$. Stresses are under safe limit considering the thickness of thermo-electric elements of $15 \mu \mathrm{m}$. 
The principal stress distribution across the TE cooler headers is plotted as shown in figures $5.99-5.101$.

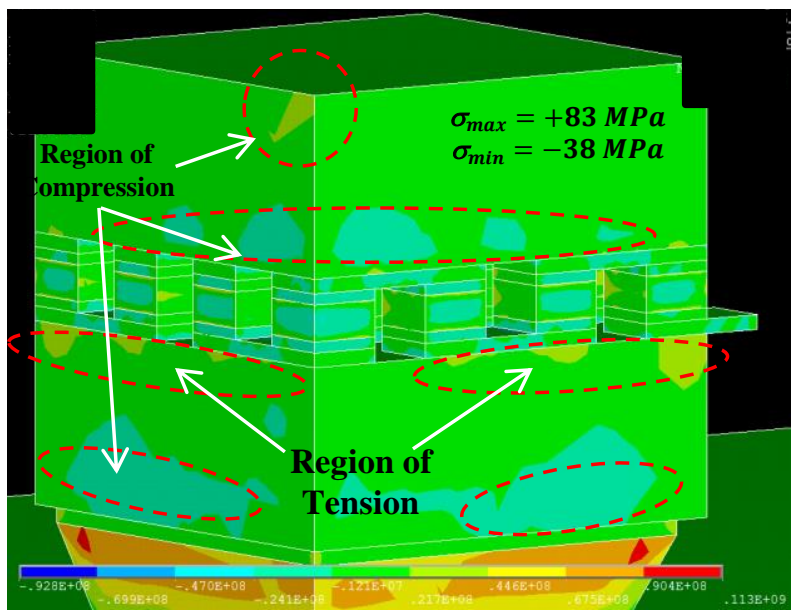

Figure 5.99: $1^{\text {st }}$ Principal Stress Plot - TE Cooler Headers

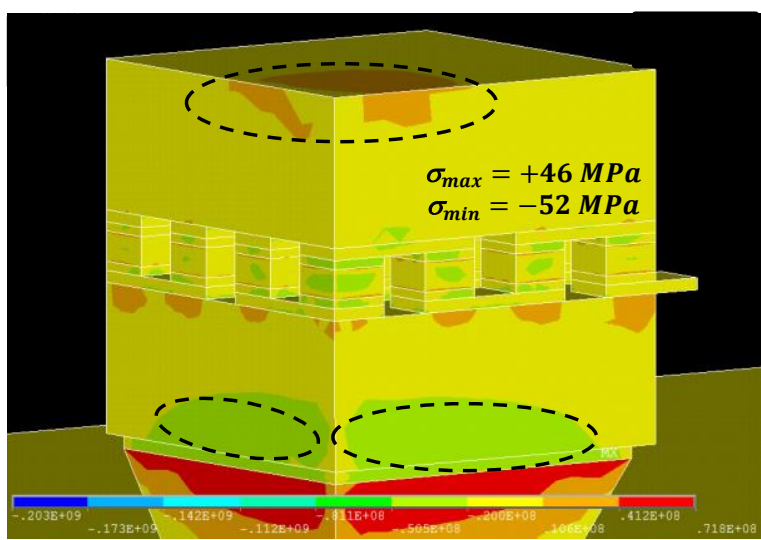

Figure 5.100: $2^{\text {nd }}$ Principal Stress Plot - TE Cooler Headers

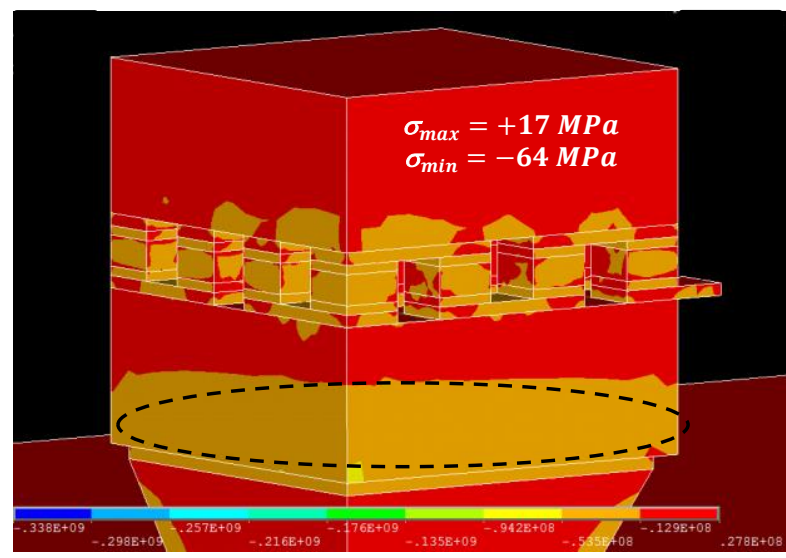

Figure 5.101: $3^{\text {rd }}$ Principal Stress Plot - TE Cooler Headers 
For a maximum principal stress of $83 \mathrm{MPa}, \mathrm{Y}=1.2$ and $\mathrm{K}_{\mathrm{IC}}$ of $2.6 \mathrm{MPa}^{*} \mathrm{~m}^{1 / 2}$, a critical flaw size of $217 \mu \mathrm{m}$ was determined based on linear elastic fracture mechanics theory.

\subsubsection{Critical Flaw Size - GaN on SiC substrate}

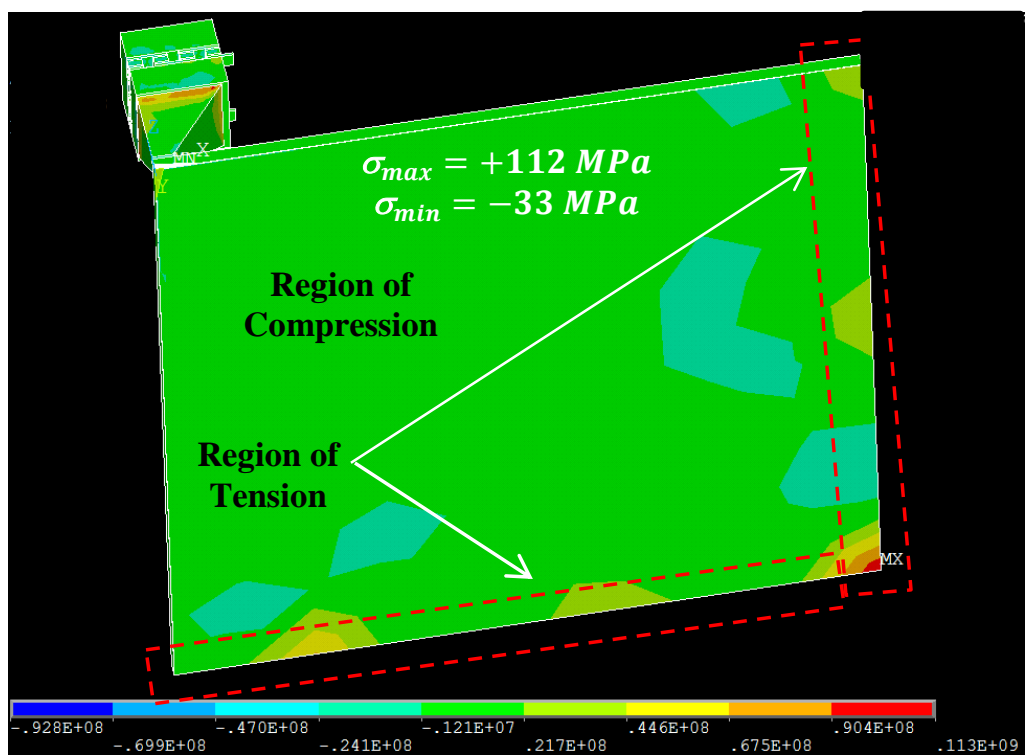

Figure 5.102: $1^{\text {st }}$ Principal Stress Plot - GaN on SiC substrate

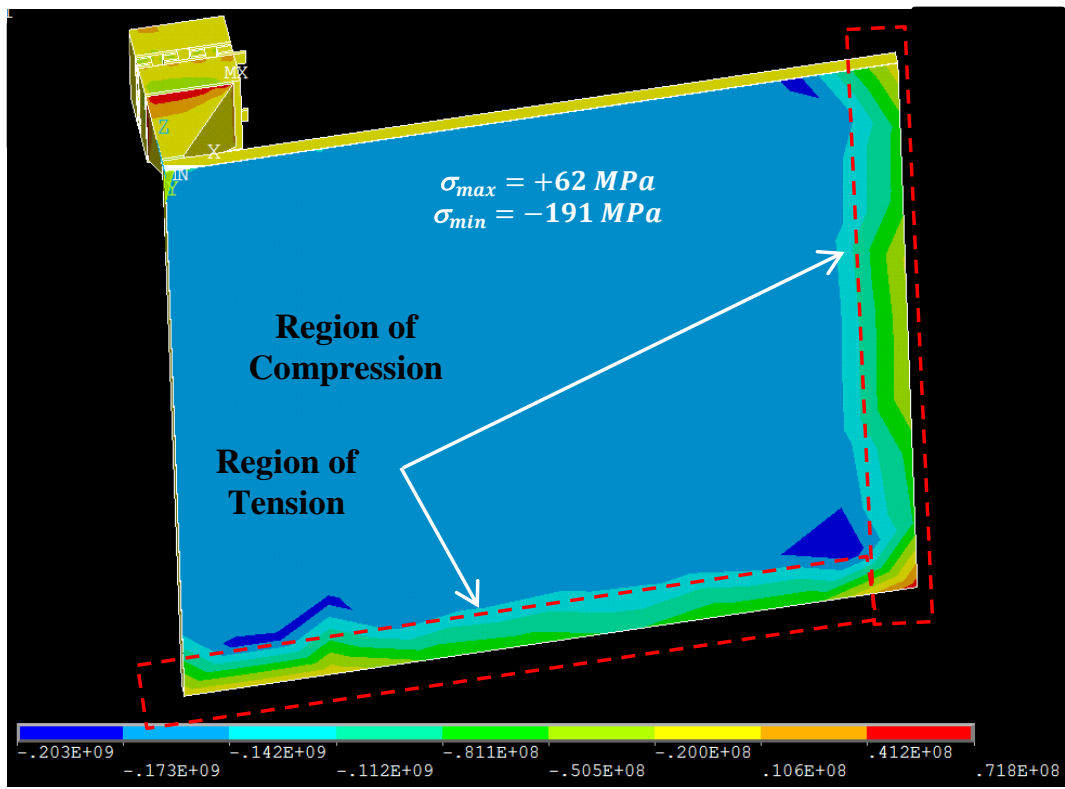

Figure 5.103: $2^{\text {nd }}$ Principal Stress Plot - GaN on SiC substrate 


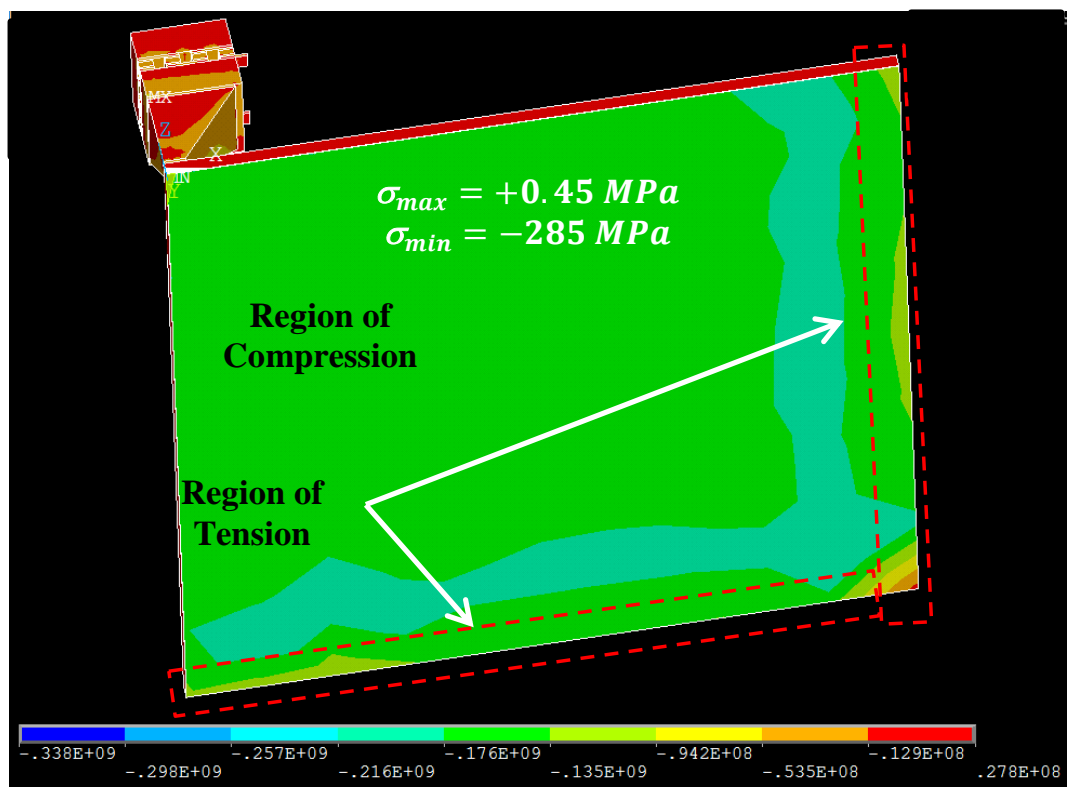

Figure 5.104: $3^{\text {rd }}$ Principal Stress Plot - SiC/GaN Device

Table 5.40: Principal Stresses - GaN on SiC substrate

\begin{tabular}{|c|c|c|c|}
\hline Parameter & $\begin{array}{c}\mathbf{1}^{\text {st }} \text { Principal } \\
\text { Stress }\end{array}$ & $\begin{array}{c}\mathbf{2}^{\text {nd }} \text { Principal } \\
\text { Stress }\end{array}$ & $\begin{array}{c}\mathbf{3}^{\text {rd }} \text { Principal } \\
\text { Stress }\end{array}$ \\
\hline Maximum & $\mathbf{1 1 2} \mathbf{M P a}$ & $62 \mathrm{MPa}$ & $0.45 \mathrm{MPa}$ \\
\hline Node Number & 180790 & 180790 & 180790 \\
\hline Minimum & $-33 \mathrm{MPa}$ & $-191 \mathrm{MPa}$ & $\mathbf{- 2 8 5} \mathbf{M P a}$ \\
\hline Node Number & 2919 & 181132 & 2919 \\
\hline
\end{tabular}

A critical flaw size of $21 \mu \mathrm{m}$ was determined for a maximum principal stress of magnitude $112 \mathrm{MPa}, \mathrm{Y}=1.2$ and $\mathrm{K}_{\mathrm{IC}}$ of $1.1 \mathrm{MPa}^{*} \mathrm{~m}^{1 / 2}$. Considering the size of micro-channel cooler, the critical flaw size indicates that the $\mathrm{GaN}$ device may fail by brittle fracture. The GaN device is also under compression near the hot-spot (Minicontact structure) and a stress of -285 $\mathrm{MPa}$ was determined in this region. It is suspected that it will affect the device performance by reverse piezoelectric effect. 


\subsection{Physics of Failure: Integrated SiC Mini-contact Model}

5.8.1 C.T.E. Mismatch: SiC Mini-contact - AlN header

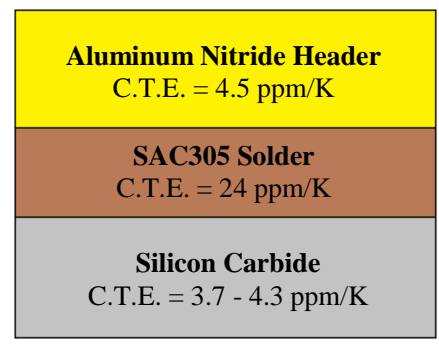

Figure 5.105: C.T.E. Mismatch: SiC - SAC305 - AlN

As shown above, there is local C.T.E. mismatch between SiC - SAC305 and SAC305 - AlN. There is negligible effect from global C.T.E. mismatch between AlN and SiC. Thus, integrated SiC Mini- contact/Cooler integration is most reliable option.

\subsubsection{C.T.E. Mismatch: Thermo-electric Cooler}

\begin{tabular}{|c|c|}
\hline $250 \mu \mathrm{m}$ & AIN header \\
\hline $10-20 \mu \mathrm{m}$ & Copper \\
\hline $10-20 \mu \mathrm{m}$ & Solder Attach \\
\hline $40-60 \mu \mathrm{m}$ & $\begin{array}{l}\text { N/P type Elements } \\
\text { Bismuth Telluride }\end{array}$ \\
\hline $10-20 \mu \mathrm{m}$ & Solder Attach \\
\hline $10-20 \mu \mathrm{m}$ & Copper \\
\hline $250 \mu \mathrm{m}$ & AIN header \\
\hline
\end{tabular}

Figure 5.106: C.T.E. Mismatch across TE Cooler

Inside the thermo-electric cooler, there is local C.T.E. mismatch between AlN (4.5 ppm/K), Copper (16.4 ppm/K), SAC305 solder (24 ppm/K), and Bismuth Telluride (18 ppm/K). As a result, we see plastic deformation in Copper and SAC305 solder attach. On a global scale, all the packaging materials are sandwiched between the AlN headers; hence, the reliability of solder is good. However, there is effect from 
local C.T.E. mismatch and temperature difference between the top and bottom headers, which contributes to the failure of solder joint.

\subsection{Physics of Failure: Discrete Copper Mini-contact Model}

\subsubsection{C.T.E. Mismatch: SiC - SAC305 - Copper}

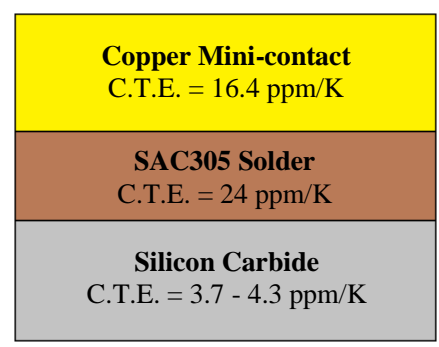

Figure 5.107: C.T.E. Mismatch: SiC - SAC305 - Copper

There is C.T.E. mismatch between SAC305 and SiC. Also, there is global C.T.E. mismatch between Copper and SiC. The C.T.E. mismatch between $\mathrm{SiC}$ and Copper results in stress generation. These stresses are above the yield point of SAC 305 and also result in plastic strains in Copper. The reason for high reliability of SAC305 at chip level integration is due to presence of Copper, which is a ductile material and supports SAC305 solder in plastic deformation process.

5.9.2 C.T.E. Mismatch: Copper - SAC305 - AlN

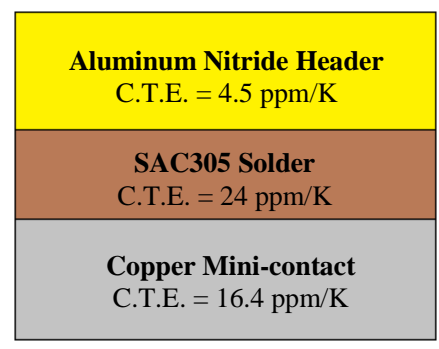

Figure 5.108: C.T.E. Mismatch: Copper - SAC305 - AlN 
At the integration level between AlN and Copper Mini-contact, there is a C.T.E. mismatch between $\mathrm{AlN}(4.5 \mathrm{ppm} / \mathrm{K})$ and $\mathrm{Cu}(16.54 \mathrm{ppm} / \mathrm{K})$. As a result, SAC305 solder fails.

\subsection{Physics of Failure: Discrete Diamond Mini-contact Model}

5.10.1 C.T.E. Mismatch: SiC - SAC305 - Diamond

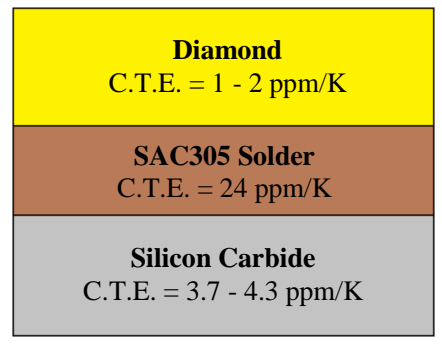

Figure 5.109: C.T.E. Mismatch: SiC - SAC305 - Diamond

From figure 5.109 above, it can be inferred that there is a small C.T.E.

mismatch between Diamond and $\mathrm{SiC}$ that can result in failure of the solder joint.

5.10.2 C.T.E. Mismatch: Diamond - SAC305 - AlN

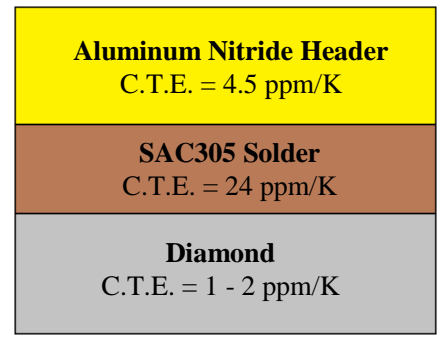

Figure 5.110: C.T.E. Mismatch: Diamond - SAC305 - AlN

The C.T.E. mismatch between AlN, SAC305 and Diamond results in failure of solder joint.

Furthermore, diamond is a stiff material and will not share the plastic strain with the SAC305 solder (ductile). As a result, if one compares the MTTF values 
determined for a diamond Mini-contact with that for a Copper Mini-contact, MTTF results for Copper are higher. This can be attributed to the fact that the Copper shares the plastic strain with the SAC305, absorbing a part of the stress load.

\subsection{Role of Geometry in Failure Process}

In this section, the effect of T-shaped, Lofted and Taper geometries on the life time (or plastic strain) of SAC305 solder will be explained.

\subsubsection{Geometry Effects on Reliability}

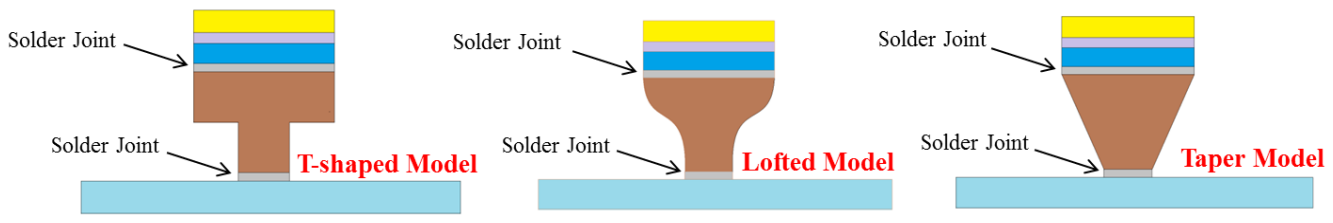

Figure 5.111: T-shaped, Lofted and Taper Mini-contact Model

For chip level integration, it was determined that T-shaped Mini-contact model is most reliable option followed by Lofted and Taper model. The bottom base of the T-shaped Mini-contact remains constant in cross-sectional area for half the height of Mini-contact to the top base. The constant cross-sectional area essentially provides the ability for sharing plastic strain with the SAC solder at chip level. This is a similar condition for the lofted model and hence, we get MTTF values at almost similar levels. However, for a taper model, the cross-sectional area from base of the Mini-contact to the top edge increases at a constant rate. From a structural standpoint, this makes a stiffer Mini-contact, leading to a higher share of the strain being resident in the solder at the base, resulting in an early failure of the SAC305 solder. 
For contact-cooler integration, the above situation is reversed. The constant slope of the tapered Mini-contact reduces the cross-sectional area near the top of the Mini-contact making it more compliant and thus sharing more of the plastic strain, whereas the larger constant cross-section of the lofted and T-shaped Mini-contacts sheds most of the plastic strain to the SAC 305 solder resulting in shorter life. The effect of geometry on SAC305 solder joint reliability inside the TE Cooler is minimal and is controlled by the type of Mini-contact material. A Copper Mini-contact shares much more of the strain as compared to a Diamond Mini-contact. Hence, the higher reliability for the three cases of Copper Mini-contact. 


\section{Chapter 6: Silver-Diamond Composite as Mini-contact Material}

The integration of thermo-electric Mini-contact based cooling in the power electronics package appears to be most reliable with an integrated SiC Mini-contact structure. With such an integration option, as opposed to a copper discrete or diamond discrete Mini-contact, the failure site shifts inside the thermo-electric cooler. In this chapter, thermo-mechanical stress analysis of Silver-Diamond composite Minicontact will be presented.

Silver-Diamond composite is a novel packaging material for next generation power electronics. It has advantages of high thermal conductivity and tailored Coefficient of Thermal Expansion (C.T.E.). Also, with use of Silver-Diamond composite as a packaging material, one can take advantage of the sintered silver phase for attachment with substrate/device. Essentially, this means the elimination of the solder joint layer for Mini-contact integration with $\mathrm{SiC}$ chip and thermo-electric cooler, leading to a reduction in thermal resistance and potentially increased reliability. . However, it's important to address heat transfer across the boundaries (diamond - silver).

Thermo-mechanical stress analysis for three different integration options of silver-diamond Mini-contacts (viz. t-shaped, lofted and taper Mini-contact) will be presented in this chapter. The linear elastic properties presented earlier in chapter 3 were input to the FE model. These properties are shown below in table 6.1. The model setup (mesh, boundary conditions) is the same as that presented in chapter 4 . The only change implemented to the geometry was removal of solder joint attachment. 
Earlier in chapter 3, elastic properties of 60\% Diamond - 40\% Silver composite were determined. The same properties were input to the Finite Element (FE) model and are presented as shown in table 6.1 below.

Table 6.1: 60\% Diamond - 40\% Silver Composite Mechanical Properties

\begin{tabular}{|c|c|}
\hline Property & Value \\
\hline Elastic Modulus & $634 \mathrm{GPa}$ \\
\hline Poisson's Ratio & 0.21 \\
\hline $\begin{array}{c}\text { Coefficient of Thermal } \\
\text { Expansion (C.T.E.) }\end{array}$ & $8.6 \mathrm{ppm} / \mathrm{K}$ \\
\hline Thermal Conductivity & $834 \mathrm{~W} / \mathrm{m}-\mathrm{K}$ \\
\hline
\end{tabular}

\subsection{T-shaped Mini-contact: Silver-Diamond Composite}

\subsubsection{Temperature Contour Plot}

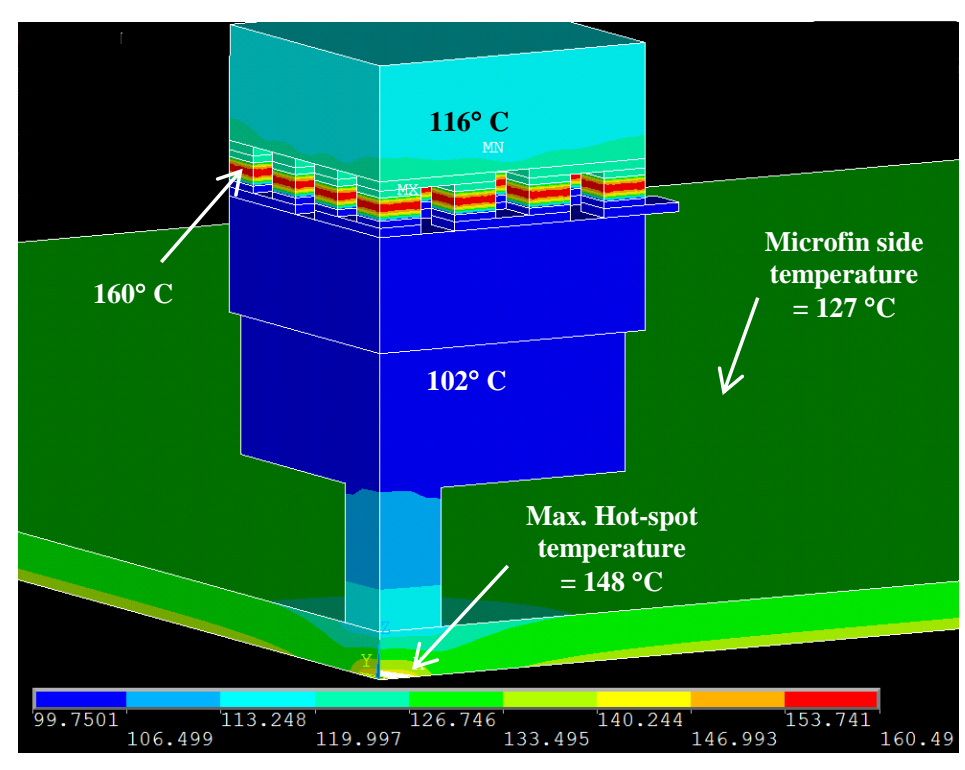

Figure 6.1: Temperature Contour Plot

The temperature distribution across the Micro-fin structure is as shown in

figure 6.1 above. A maximum hot-spot temperature of $148{ }^{\circ} \mathrm{C}$ was determined through Finite Element simulations. Temperature on Micro-fin side was determined 
to be $127^{\circ} \mathrm{C}$ and a maximum temperature of $160{ }^{\circ} \mathrm{C}$ was determined for thermoelectrics.

\subsubsection{Displacement Vector Plot}

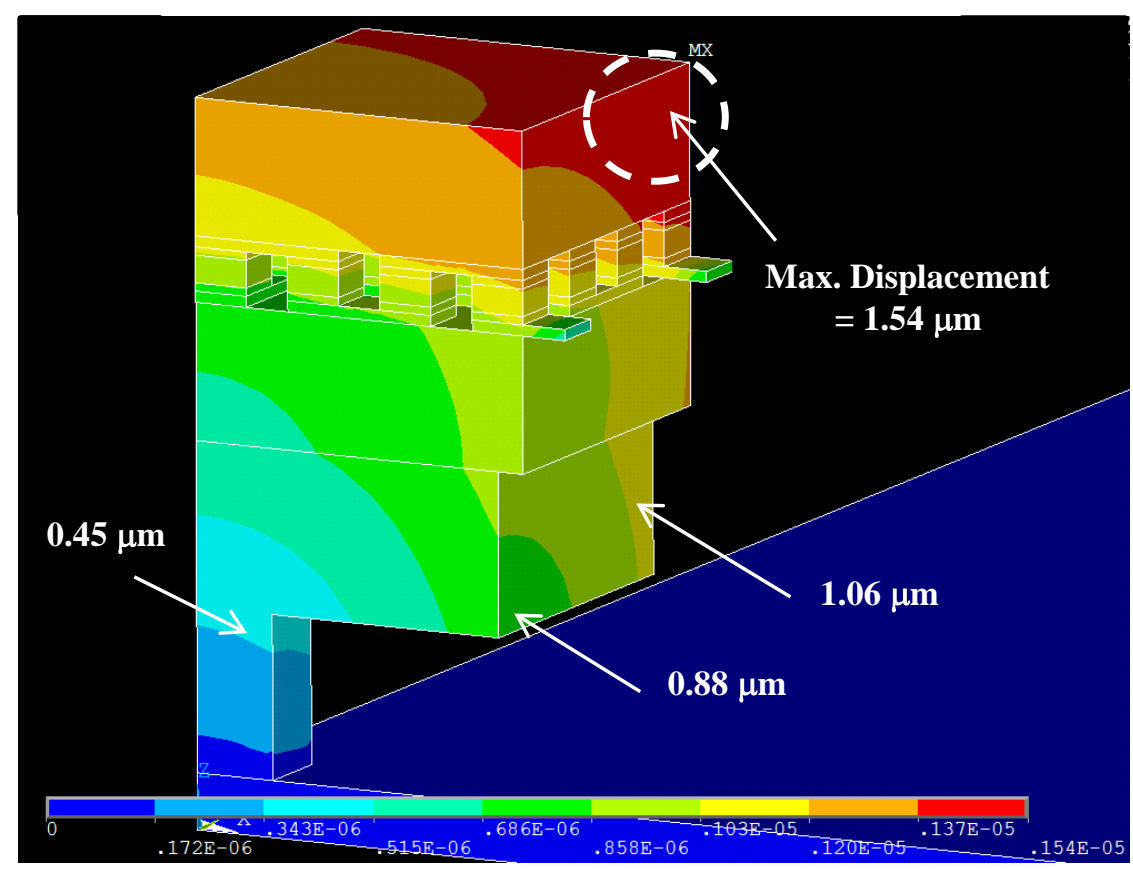

Figure 6.2: Displacement Contour Plot

A maximum displacement of $1.54 \mu \mathrm{m}$ was determined for the top edge of the TE Cooler. There is no displacement/deformation in $\mathrm{SiC}$ substrate region. A deformation in the range of $0.45 \mu \mathrm{m}-1.06 \mu \mathrm{m}$ was determined for the Mini-contact region.

\subsubsection{Thermo-electric Cooler Reliability}

The failure of solder joints interconnecting thermo-electric elements with copper-metallized headers (AIN) was determined from thermo-mechanical modeling. It was found through analysis that the solder joint on the top side of the cooler is under a plastic strain of $8.64 \times 10^{-3}$. The joint to the lower header is at a 
comparatively lower temperature and experiences lower strains. The solder joint MTTF was calculated based on Engelmaier's failure model.

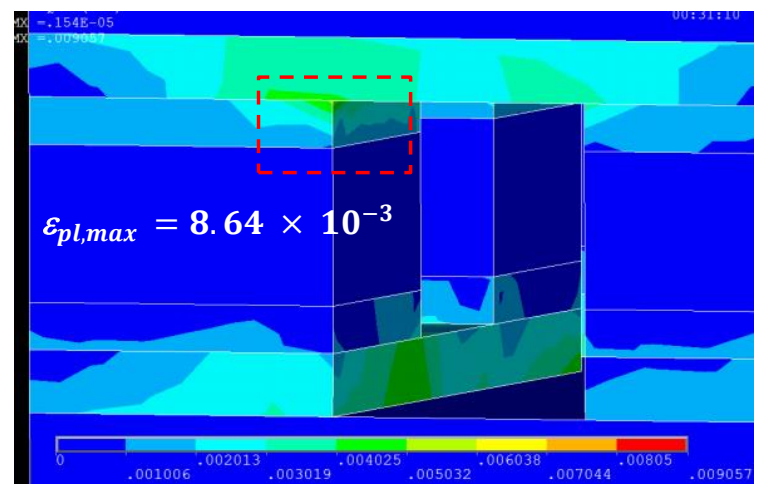

Figure 6.3: Pl. Strain - Solder Joint: Thermo-electric Cooler

Table 6.2: Design for Reliability (MTTF) - Solder Joint Thermo-electric Cooler

\begin{tabular}{|c|c|}
\hline Maximum Plastic Strain & $8.64 \mathrm{e}-3$ \\
\hline Maximum Temperature & $122^{\circ} \mathrm{C}$ \\
\hline Minimum Temperature & $25^{\circ} \mathrm{C}$ \\
\hline Average Temperature & $73.54{ }^{\circ} \mathrm{C}$ \\
\hline Node Number & 114333 \\
\hline MTTF (596 mins.) & $\mathbf{2 , 6 0 8}$ cycles \\
\hline MTTF (20 mins.) & $\mathbf{5 , 8 2 0}$ cycles \\
\hline MTTF (1 min.) & $\mathbf{2 3 , 3 4 2}$ cycles \\
\hline
\end{tabular}

An MTTF of 2,608 cycles, 5,820 cycles and 23,342 cycles was determined for solder joint inside the thermo-electric cooler.

\subsubsection{Stress Distribution across Mini-contact}

Since elastic-plastic properties of $60 \%$ Diamond - 40\% Silver are unavailable in literature; an elastic stress analysis was used to determine the failure site or region of stress concentration in Mini-contact at which failure will occur. Through analysis, one can predict that the maximum stress site will be the Mini-contact region near the SiC chip integration. An elastic stress of $2.83 \mathrm{GPa}$ was determined through thermomechanical modeling. The same is plotted as shown in figure 6.3 below. 


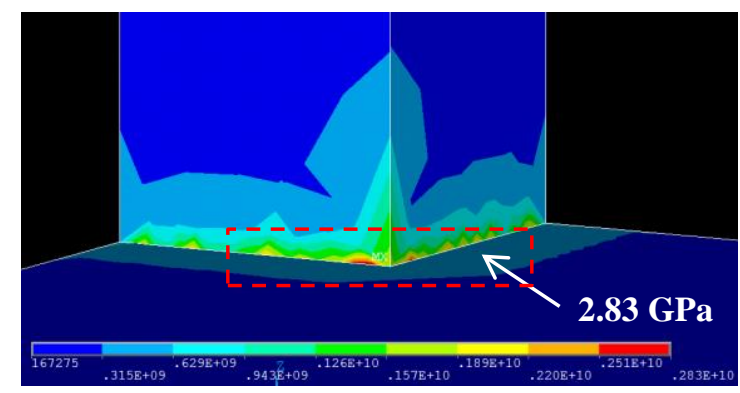

Figure 6.4: Von-Mises Stress Distribution: Mini-contact Region

6.1.5 Critical Flaw Size - Thermo-electric Headers and Elements

Table 6.3: Principal Stresses - Thermoelectric Header

\begin{tabular}{|c|c|c|c|}
\hline Parameter & $\begin{array}{c}\mathbf{1}^{\text {st }} \text { Principal } \\
\text { Stress }\end{array}$ & $\begin{array}{c}\mathbf{2}^{\text {nd }} \text { Principal } \\
\text { Stress }\end{array}$ & $\begin{array}{c}\mathbf{3}^{\text {rd }} \text { Principal } \\
\text { Stress }\end{array}$ \\
\hline Maximum & $\mathbf{1 3 6} \mathrm{MPa}$ & $117 \mathrm{MPa}$ & $30 \mathrm{MPa}$ \\
\hline Node Number & 103354 & 53111 & 153335 \\
\hline Minimum & $-47 \mathrm{MPa}$ & $-145 \mathrm{MPa}$ & $\mathbf{- 1 5 2} \mathbf{M P a}$ \\
\hline Node Number & 105085 & 53103 & 53105 \\
\hline
\end{tabular}

The critical flaw size for a maximum stress of $136 \mathrm{MPa}$ can be determined

from linear elastic fracture mechanics as $81 \mu \mathrm{m}$ for a $\mathrm{Y}=1.2$ and $\mathrm{K}_{\mathrm{IC}}$ of 2.6

$\mathrm{MPa}^{*} \mathrm{~m}^{1 / 2}$. The flaw size is relatively large and the stresses are under safe limit.

The maximum principal stresses in thermo-electric elements are as shown

below in table 6.3 .

Table 6.4: Principal Stresses - Thermoelectric Elements

\begin{tabular}{|c|c|c|c|}
\hline Parameter & $\begin{array}{c}\mathbf{1}^{\text {st }} \text { Principal } \\
\text { Stress }\end{array}$ & $\begin{array}{c}\mathbf{2}^{\text {nd }} \text { Principal } \\
\text { Stress }\end{array}$ & $\begin{array}{c}\mathbf{3}^{\text {rd }} \text { Principal } \\
\text { Stress }\end{array}$ \\
\hline Maximum & $\mathbf{7 4} \mathbf{M P a}$ & $52 \mathrm{MPa}$ & $25 \mathrm{MPa}$ \\
\hline Node Number & 90595 & 83068 & 90221 \\
\hline Minimum & $-27 \mathrm{MPa}$ & $-43.54 \mathrm{MPa}$ & $-59 \mathrm{MPa}$ \\
\hline Node Number & 83068 & 90920 & 83070 \\
\hline
\end{tabular}


Critical flaw size in $\mathrm{Bi}_{2} \mathrm{Te}_{3}$ elements for a maximum stress of $74 \mathrm{MPa}$ was found out to be in the range of $40 \mu \mathrm{m}-162 \mu \mathrm{m}$ for a $\mathrm{Y}=1.2$ and $\mathrm{K}_{\mathrm{IC}}$ in range of 1 $\mathrm{MPa}^{*} \mathrm{~m}^{1 / 2}-2 \mathrm{MPa}^{*} \mathrm{~m}^{1 / 2}$. The flaw size is relatively large and thus, stresses are safe.

\subsection{Lofted Mini-contact: Silver-Diamond Composite}

\subsubsection{Temperature Contour Plot}

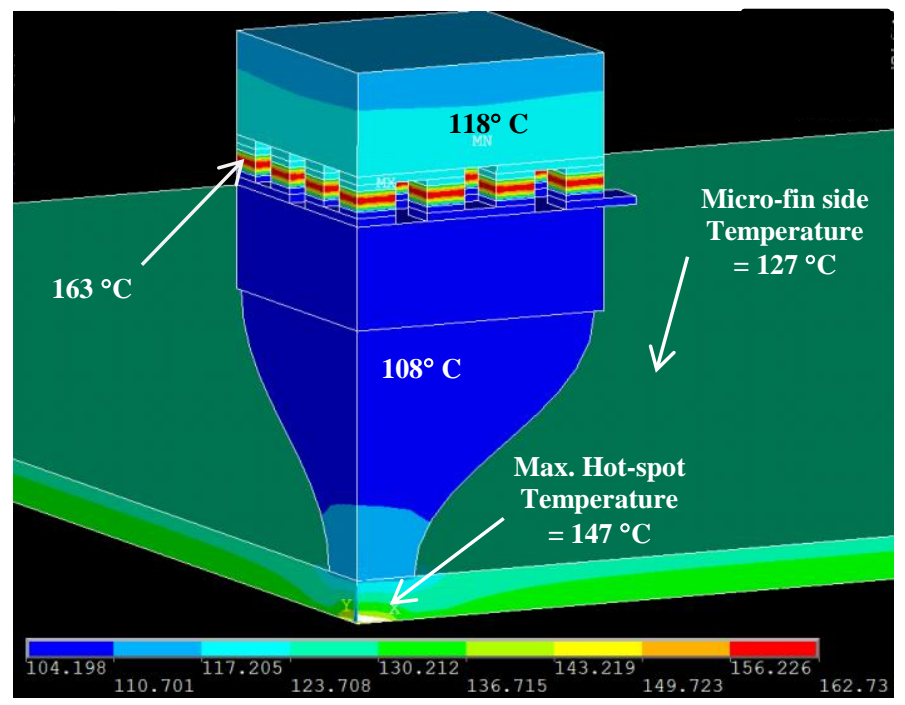

Figure 6.5: Nodal Temperature Contour Plot

A maximum hot-spot temperature of $147^{\circ} \mathrm{C}$ was determined for the lofted Mini-contact model. Micro-fin side temperature was found to be $127{ }^{\circ} \mathrm{C}$ and the hottest region of the package was as usual, the thermo-electric elements with a temperature of $163^{\circ} \mathrm{C}$. 


\subsubsection{Displacement Contour Plot}

The deformation across the lofted Mini-contact and cooler structure is as shown in figure 6.7 below. A maximum displacement of $1.53 \mu \mathrm{m}$ was seen at the edge of the thermo-electric cooler.

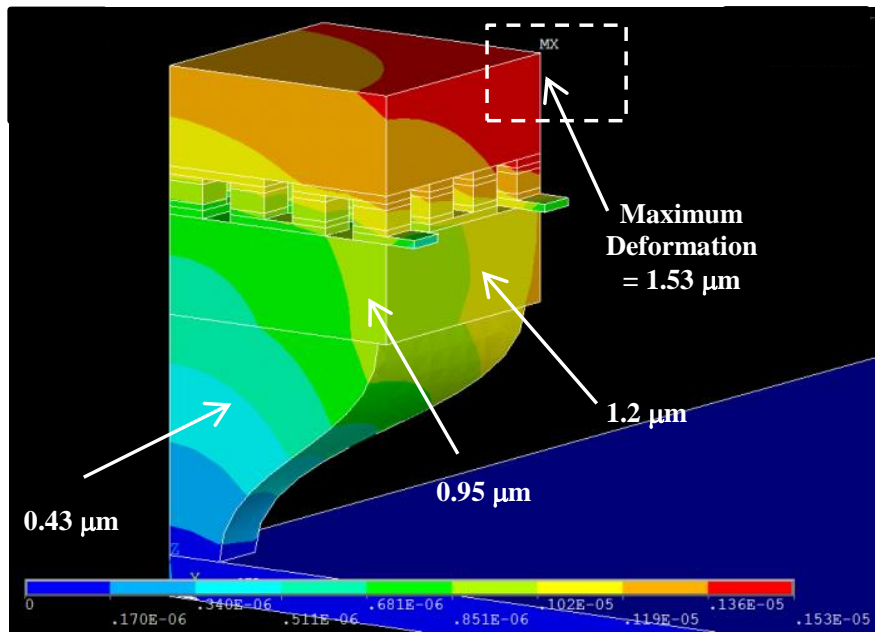

Figure 6.6: Displacement Contour Plot

\subsubsection{Thermo-electric Cooler Reliability}

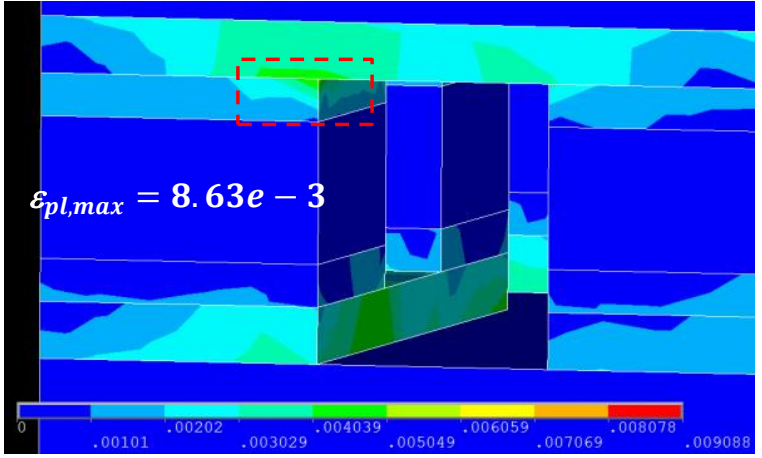

Figure 6.7: Pl. Strain - Solder Joint Thermo-electric Cooler

For a Von-Mises plastic strain of $8.63 \times 10^{-3}$, the MTTF of solder joint can be calculated based on the Engelmaier's failure model. It is shown in table 6.4 below. 
Table 6.5: Design for Reliability (MTTF) - Solder Joint Thermo-electric Cooler

\begin{tabular}{|c|c|}
\hline Maximum Plastic Strain & $8.63 \mathrm{e}-3$ \\
\hline Maximum Temperature & $122.21^{\circ} \mathrm{C}$ \\
\hline Minimum Temperature & $25^{\circ} \mathrm{C}$ \\
\hline Average Temperature & $73.61^{\circ} \mathrm{C}$ \\
\hline Node Number & 121974 \\
\hline MTTF (596 mins.) & $\mathbf{2 , 6 1 2}$ cycles \\
\hline MTTF (20 mins.) & $\mathbf{5 , 0 2 2}$ cycles \\
\hline MTTF (1 min.) & $\mathbf{2 0 , 1 4 2}$ cycles \\
\hline
\end{tabular}

An MTTF of 2,612 cycles, 5,022 cycles and 20,142 cycles was determined for the solder joint interconnecting thermo-electrics to top side AlN header.

\subsubsection{Stress Distribution across Mini-contact}

The stress distribution across the lofted Mini-contact structure is as shown below in figure 6.8 .

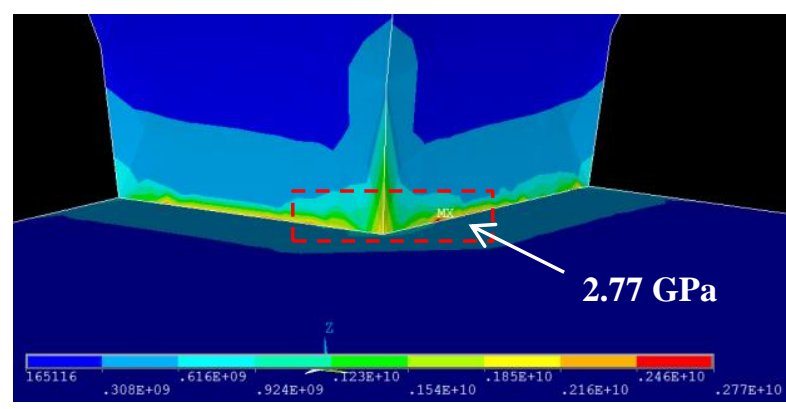

Figure 6.8: Von-Mises Stress Distribution: Mini-contact Region

A maximum stress of $2.77 \mathrm{GPa}$ was determined through FE simulations located near the SiC substrate region. 
6.2.5 Critical Flaw Size - Thermoelectric Elements and Headers

Table 6.6: Principal Stresses - Thermoelectric Elements

\begin{tabular}{|c|c|c|c|}
\hline Parameter & $\begin{array}{c}\mathbf{1}^{\text {st }} \text { Principal } \\
\text { Stress }\end{array}$ & $\begin{array}{c}\mathbf{2}^{\text {nd }} \text { Principal } \\
\text { Stress }\end{array}$ & $\begin{array}{c}\mathbf{3}^{\text {rd }} \text { Principal } \\
\text { Stress }\end{array}$ \\
\hline Maximum & $\mathbf{7 2 ~ M P a}$ & $52 \mathrm{MPa}$ & $24 \mathrm{MPa}$ \\
\hline Node Number & 98236 & 90709 & 97862 \\
\hline Minimum & $-27 \mathrm{MPa}$ & $-43 \mathrm{MPa}$ & $-59 \mathrm{MPa}$ \\
\hline Node Number & 90709 & 98561 & 90711 \\
\hline
\end{tabular}

Table 6.7: Principal Stresses - Thermoelectric Headers

\begin{tabular}{|c|c|c|c|}
\hline Parameter & $\begin{array}{c}\mathbf{1}^{\text {st }} \text { Principal } \\
\text { Stress }\end{array}$ & $\begin{array}{c}\mathbf{2}^{\text {nd }} \text { Principal } \\
\text { Stress }\end{array}$ & $\begin{array}{c}\mathbf{3}^{\text {rd }} \text { Principal } \\
\text { Stress }\end{array}$ \\
\hline Maximum & $\mathbf{1 3 3} \mathbf{M P a}$ & $115 \mathrm{MPa}$ & $29 \mathrm{MPa}$ \\
\hline Node Number & 110995 & 50245 & 161535 \\
\hline Minimum & $-47 \mathrm{MPa}$ & $-148 \mathrm{MPa}$ & $\mathbf{- 1 8 7} \mathbf{M P a}$ \\
\hline Node Number & 112726 & 50237 & 49972 \\
\hline
\end{tabular}

Thermo-electric Elements, flaw size: $43 \mu \mathrm{m}$ - $171 \mu \mathrm{m}$, Headers, flaw size: $85 \mu \mathrm{m}$

Stresses are under safe limit (assumed same conditions/properties as last case).

\subsection{Taper Mini-contact: Silver-Diamond Composite}

6.3.1 Temperature Contour Plot

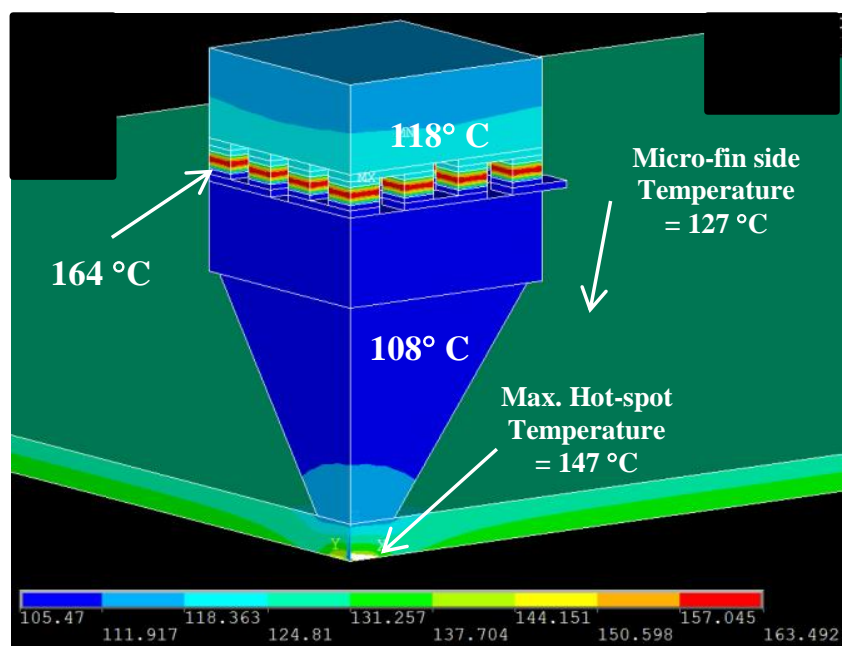

Figure 6.9: Temperature Contour Plot across Model 


\subsubsection{Displacement Contour Plot}

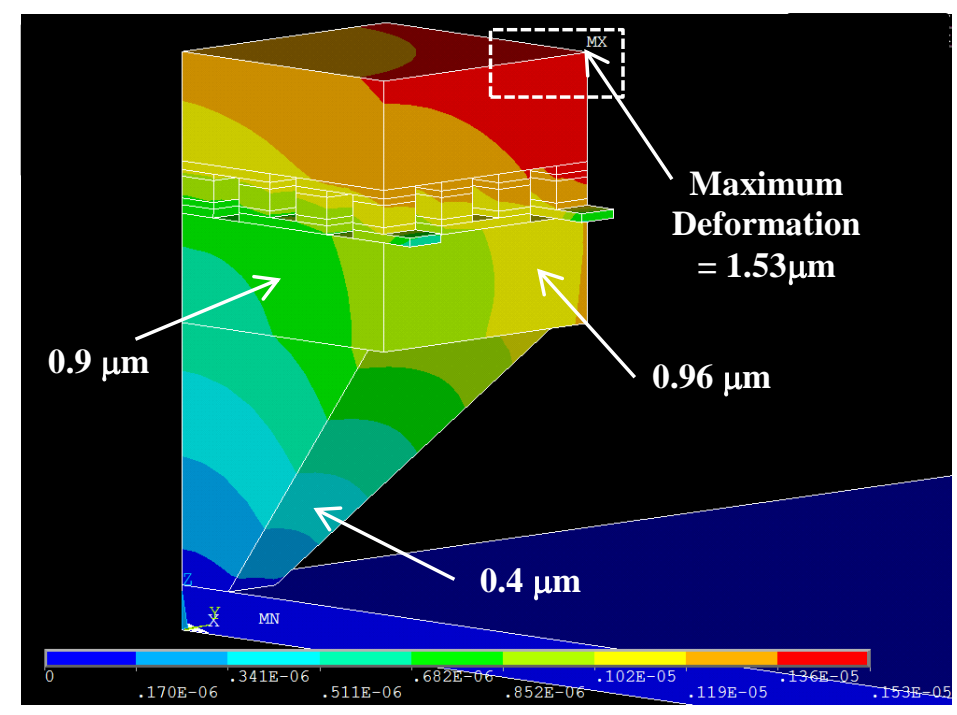

Figure 6.10: Displacement Contour Plot

\subsubsection{Thermo-electric Cooler Reliability}

The Mean Time to Failure (MTTF) for a plastic strain of $8.73 \times 10^{-3}$ was calculated based on Engelmaier's failure model, as shown in table 6.7 below.

Table 6.8: Design for Reliability (MTTF) - Thermo-electric Cooler Reliability

\begin{tabular}{|c|c|}
\hline Maximum Plastic Strain & $8.73 \mathrm{e}-3$ \\
\hline Maximum Temperature & $122.26^{\circ} \mathrm{C}$ \\
\hline Minimum Temperature & $25^{\circ} \mathrm{C}$ \\
\hline Average Temperature & $73.63^{\circ} \mathrm{C}$ \\
\hline Node Number & 113011 \\
\hline MTTF $(\mathbf{5 9 6}$ mins.) & $\mathbf{2 , 5 4 5}$ cycles \\
\hline MTTF $(\mathbf{2 0}$ mins.) & $\mathbf{4 , 8 8 5}$ cycles \\
\hline MTTF $(\mathbf{1}$ min.) & $\mathbf{1 9 , 5 1 1 ~ c y c l e s ~}$ \\
\hline
\end{tabular}

A MTTF of 2,545 cycles, 4,885 cycles and 19,511 cycles was determined for solder joint interconnecting thermo-electric elements with Copper-metallized AlN headers. 
6.3.4 Stress Distribution across Mini-contact structure

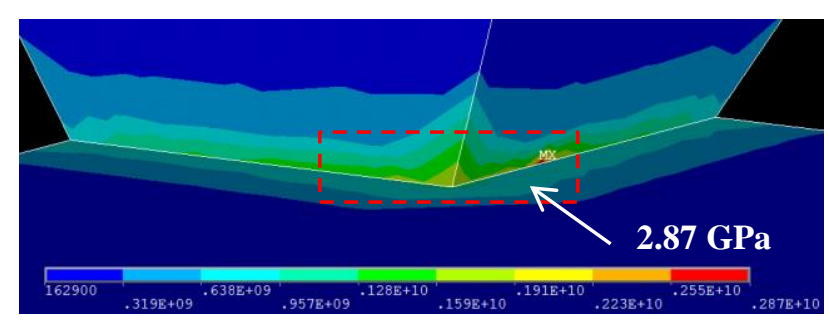

Figure 6.11: Von-Mises Stress Contour Plot

Figure 6.13 gives stress distribution in Mini-contact structure near the $\mathrm{SiC}$ chip region. A maximum stress of $2.87 \mathrm{GPa}$ was determined at the edge of Minicontact structure.

6.3.5 Critical Flaw Size - Thermoelectric Elements and Headers

Table 6.9: Principal Stresses - Thermoelectric Headers

\begin{tabular}{|c|c|c|c|}
\hline Parameter & $\begin{array}{c}\mathbf{1}^{\text {st }} \text { Principal } \\
\text { Stress }\end{array}$ & $\begin{array}{c}\mathbf{2}^{\text {nd }} \text { Principal } \\
\text { Stress }\end{array}$ & $\begin{array}{c}\mathbf{3}^{\text {rd }} \text { Principal } \\
\text { Stress }\end{array}$ \\
\hline Maximum & $\mathbf{1 3 7} \mathbf{M P a}$ & $123 \mathrm{MPa}$ & $30 \mathrm{MPa}$ \\
\hline Node Number & 102032 & 48486 & 148292 \\
\hline Minimum & $-47 \mathrm{MPa}$ & $-164 \mathrm{MPa}$ & $\mathbf{- 2 3 4} \mathbf{M P a}$ \\
\hline Node Number & 103763 & 48484 & 48463 \\
\hline
\end{tabular}

Table 6.10: Principal Stresses - Thermoelectric Elements

\begin{tabular}{|c|c|c|c|}
\hline Parameter & $\begin{array}{c}\mathbf{1}^{\text {st }} \text { Principal } \\
\text { Stress }\end{array}$ & $\begin{array}{c}\mathbf{2}^{\text {nd }} \text { Principal } \\
\text { Stress }\end{array}$ & $\begin{array}{c}\mathbf{3}^{\text {rd }} \text { Principal } \\
\text { Stress }\end{array}$ \\
\hline Maximum & $73 \mathrm{MPa}$ & $53 \mathrm{MPa}$ & $24 \mathrm{MPa}$ \\
\hline Node Number & 89273 & 81746 & 88899 \\
\hline Minimum & $-27 \mathrm{MPa}$ & $-43 \mathrm{MPa}$ & $-60 \mathrm{MPa}$ \\
\hline Node Number & 81746 & 89598 & 81748 \\
\hline
\end{tabular}

Thermo-electric Headers, critical flaw size: $80 \mu \mathrm{m}(\mathrm{Y}=1.2$, stress $=137 \mathrm{MPa}$ and $\mathrm{K}_{\mathrm{IC}}=2.6 \mathrm{MPa}^{*} \mathrm{~m}^{1 / 2}$ ). Thermo-electric elements, critical flaw size: $42 \mu \mathrm{m}-166$ $\mu \mathrm{m}\left(\mathrm{Y}=1.2\right.$, stress $=73 \mathrm{MPa}$ and $\left.\mathrm{K}_{\mathrm{IC}}=1 \mathrm{MPa}^{*} \mathrm{~m}^{1 / 2}-2 \mathrm{MPa}^{*} \mathrm{~m}^{1 / 2}\right)$. Stresses are under safe limit (assumed same conditions/properties as last case). 


\section{Chapter-7: Manifold Micro-channel Structural Reliability}

The two-phase micro-channel cooler aims at implementation of forced fed cooling technology [38] for next generation power electronics. A schematic of the cooler assembly is shown in figure 7.1 below. The micro-channel cooler aims at dissipating a background heat flux of $1 \mathrm{k} \mathrm{W} / \mathrm{cm}^{2}$ using a two-phase refrigerant. From a structural reliability standpoint, it was important to consider the effect of sudden change in cross-sectional area from micro-fin base region to micro-fin structure. As a first analysis, it was hypothesized that the micro-fin structure will fail by brittle fracture at the corners of micro-fin.

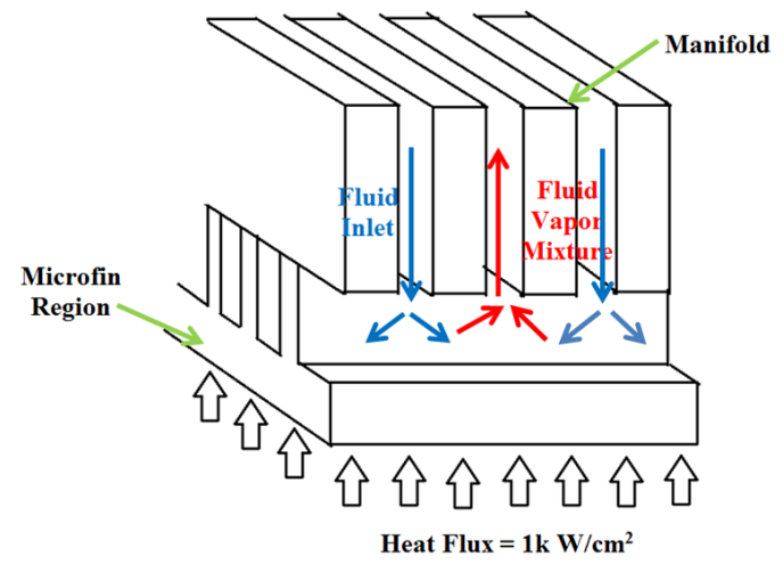

Figure 7.1: Manifold Micro-channel Cooling Schematic, [38]

The size of the outlet channel (hot fluid/vapor mixture) is about two times the dimension of the inlet channel (fluid). This helps in creating appropriate pressure drop across the channels and creating a turbulence effect, which will allow the fluidvapor mixture to flow across the outlet channel.

The advanced cooling technology aims at implementation of a micro-channel cooler with a micro-fin thickness as small as $10 \mu \mathrm{m}$. The thickness of micro-channel 
substrate was assumed to be around $400 \mu \mathrm{m}$. It was assumed that the manifold structure is interconnected to the micro-fin assembly via $20 \mu \mathrm{m}$ thick solder joint. The height of the manifold was assumed to be about $500 \mu \mathrm{m}$. Figure 7.2 gives an overview of the unit cell model.

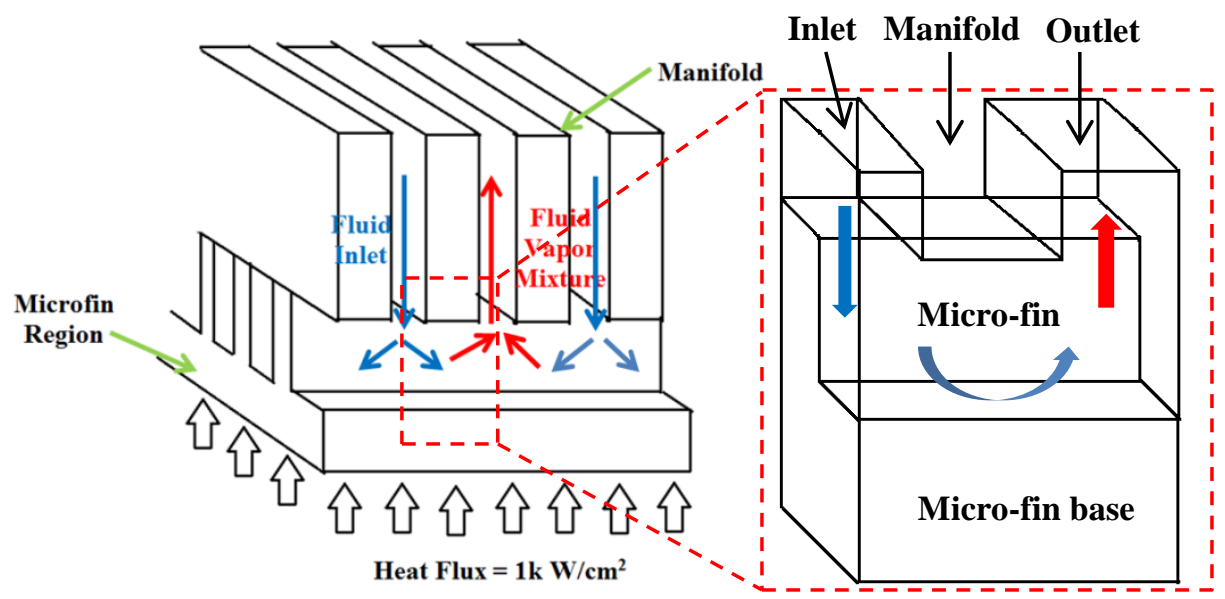

Figure 7.2: Unit Cell Model (right), [38]

As mentioned earlier, the size of the outlet port is twice the size of the inlet

port. The width of the inlet channel was set to $50 \mu \mathrm{m}$, whereas that for outlet channel was set to $100 \mu \mathrm{m}$.

\subsection{Manifold - Microchannel Two-Phase Cooling: Structural Modeling}

\subsubsection{Temperature Contour Plot}

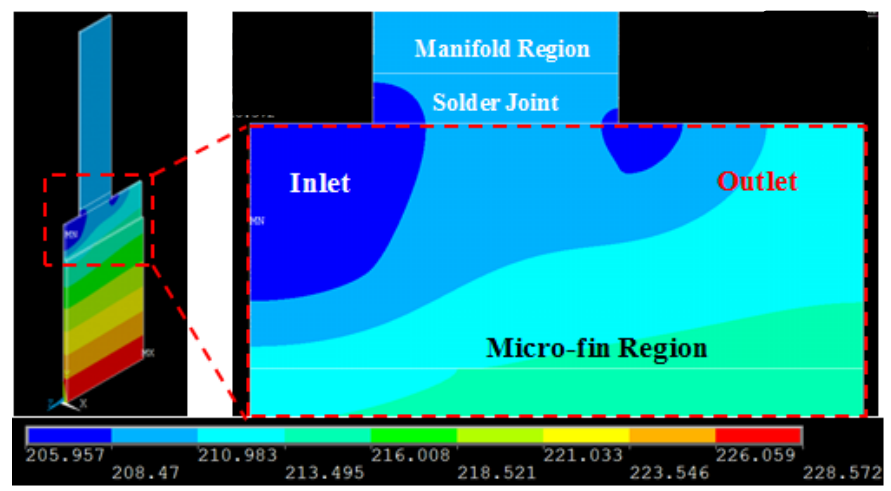

Figure 7.3: Temperature Profile across Fins, [38] Credits: Raphael Mandel 
The novel cooling technology [38] uses a fluid with a saturation temperature of $150{ }^{\circ} \mathrm{C}$. It can be inferred from figure 7.3 above that the outlet channel is at a higher temperature as compared to the inlet channel. As per the heat transfer coefficient data supplied to the model, one can observe a thin film formation across the micro-fin structure. The micro-fluidics uses a two-phase coolant for removal of heat from the SiC substrate. At the inlet port, liquid coolant is supplied to the microfin cooling region. However, at the outlet port there is combination of liquid and vapor phase. From a structural reliability standpoint, it was important to evaluate the effect of temperature drop across the micro-fin area to thermo-mechanical stress generation.

7.1.2 Critical Flaw Size - Micro-fin corner

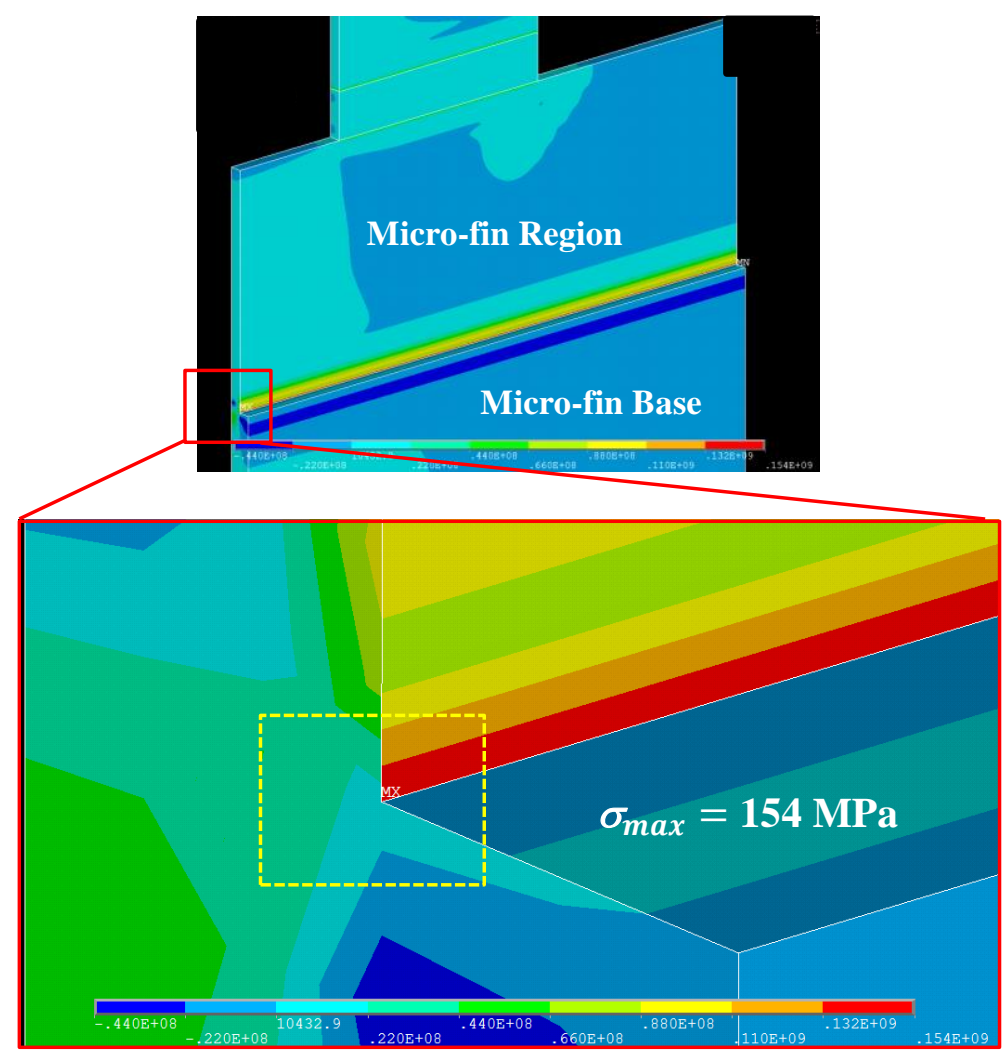

Figure 7.4: Maximum Principal Stress Plot - Micro-fin Corner 
Through thermo-mechanical stress analysis using the boundary conditions from chapter 4, a maximum principal stress of $154 \mathrm{MPa}$ was determined at the edge of the micro-fin corner as shown above in figure 7.4. The stress concentration at micro-fin and micro-channel base corner can be attributed to the temperature drop across the micro-fin profile and displacement boundary conditions. Furthermore, the fracture mechanics approach was applied to determine the critical flaw size necessary to cause propagation of micro-crack across the corner into the $\mathrm{SiC}$ substrate.

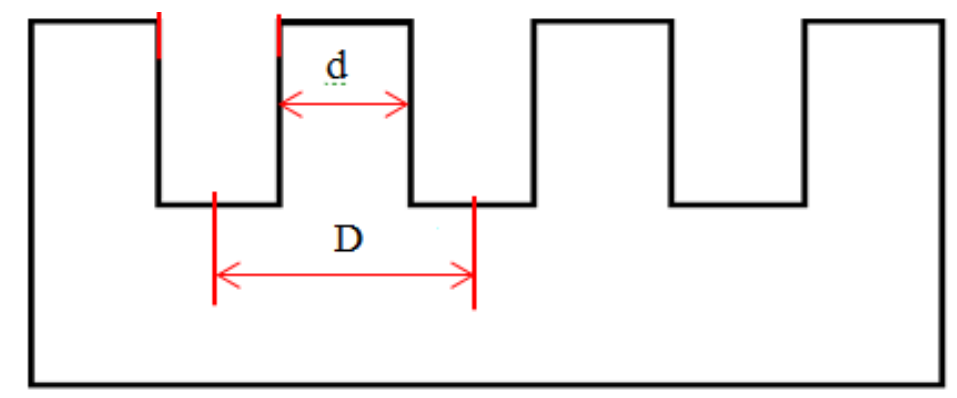

Figure 7.5: Stress Concentration Factor

As mentioned earlier, the micro-fin structure essentially resembles a situation where there is transition of cross sections (micro-channel base region to micro-fin region). There is an associated stress concentration effect, which will need to be incorporated in the calculation of critical flaw size. The sharpness of the corner will contribute to this effect. Additionally, it is important to mention here that manufacturing (etching of fins from substrate) will contribute to the sharpness of the micro-fin corner.

The stress concentration factor can be determined from charts available in literature. For a D/d ratio of 2 , the stress concentration factor can be evaluated to be in between 1 to 3 [53]. Using the fracture mechanics approach, we can evaluate critical flaw size as follows. 


$$
K_{x y z}=(1.12) * f_{s c} * \sigma_{\text {max }} * \sqrt{\pi a}
$$

$\mathrm{K}_{\mathrm{xyz}}$ is the fracture toughness value of $\mathrm{SiC}$ in the direction of crack propagation, which was determined to be [110]. The fracture toughness value in this direction is $2 \mathrm{MPa}^{*} \mathrm{~m}^{1 / 2} .1 .12$ is a factor related to the presence of crack at the outer edge of the material. Substituting all value in the above equation, the critical crack size can be determined to be in the range of $5 \mu \mathrm{m}-45 \mu \mathrm{m}$. On comparing the size of micro-channel cooler with the critical crack size determined from thermo-mechanical stress analysis, one can conclude that the stresses will assist in crack propagation but will not cause instantaneous failure at their initial levels. 


\section{Chapter 8: Conclusions - Structural Reliability Modeling}

\section{$\underline{8.1 \text { Conclusions }}$}

Table 8.1: Reliability Comparison

Integrated SiC Mini-contact and Discrete Copper Mini-contact

\begin{tabular}{|c|c|c|c|c|}
\hline \multirow[t]{2}{*}{ Parameter } & \multirow{2}{*}{$\begin{array}{l}\text { Integrated } \\
\text { SiC Mini- } \\
\text { contact }\end{array}$} & \multicolumn{3}{|c|}{$\begin{array}{l}\text { Discrete Copper } \\
\text { Mini-contact }\end{array}$} \\
\hline & & T-shaped & Lofted & Taper \\
\hline $\begin{array}{c}\text { Hot-Spot } \\
\text { Temperature }\end{array}$ & $138^{\circ} \mathrm{C}$ & $139^{\circ} \mathrm{C}$ & $138^{\circ} \mathrm{C}$ & $137{ }^{\circ} \mathrm{C}$ \\
\hline $\begin{array}{c}\text { Maximum } \\
\text { Deformation }\end{array}$ & $0.85 \mu \mathrm{m}$ & $1.33 \mu \mathrm{m}$ & $1.9 \mu \mathrm{m}$ & $1.5 \mu \mathrm{m}$ \\
\hline $\begin{array}{l}\text { Maximum } \\
\text { Deformation } \\
\text { Location }\end{array}$ & $\begin{array}{l}\text { TE Cooler } \\
\text { Header Edge }\end{array}$ & $\begin{array}{l}\text { Mini- } \\
\text { contact } \\
\text { Edge }\end{array}$ & $\begin{array}{l}\text { Solder } \\
\text { Joint Chip } \\
\text { Level }\end{array}$ & $\begin{array}{l}\text { Solder Joint } \\
\text { Chip Level }\end{array}$ \\
\hline $\begin{array}{c}\text { MTTF solder } \\
\text { Chip/Mini-contact } \\
596 \text { mins. dwell }\end{array}$ & N/A & $\begin{array}{l}39,300 \\
\text { cycles }\end{array}$ & $\begin{array}{l}38,594 \\
\text { cycles }\end{array}$ & 15,973 cycles \\
\hline $\begin{array}{c}\text { MTTF solder } \\
\text { Chip/Mini-contact } \\
20 \text { mins. dwell }\end{array}$ & N/A & $\begin{array}{l}92,447 \\
\text { cycles }\end{array}$ & $\begin{array}{l}90,783 \\
\text { cycles }\end{array}$ & 35,456 cycles \\
\hline $\begin{array}{c}\text { MTTF solder } \\
\text { Chip/Mini-contact } \\
1 \text { min. dwell }\end{array}$ & N/A & $\begin{array}{l}569,205 \\
\text { cycles }\end{array}$ & $\begin{array}{l}559,262 \\
\text { cycles }\end{array}$ & $\begin{array}{l}193,797 \\
\text { cycles }\end{array}$ \\
\hline $\begin{array}{l}\text { MTTF solder } \\
\text { Contact/Cooler } \\
596 \text { mins. dwell }\end{array}$ & 3,867 cycles & 104 cycles & 110 cycles & 308 cycles \\
\hline $\begin{array}{l}\text { MTTF solder } \\
\text { Contact/Cooler } \\
20 \text { mins. dwell } \\
\end{array}$ & 7,876 cycles & 160 cycles & 169 cycles & 512 cycles \\
\hline $\begin{array}{l}\text { MTTF solder } \\
\text { Contact/Cooler } \\
1 \text { min. dwell } \\
\end{array}$ & 36,191 cycles & 404 cycles & 426 cycles & 1,519 cycles \\
\hline $\begin{array}{c}\text { Cooler MTTF } \\
596 \text { mins. dwell }\end{array}$ & 2,039 cycles & $\begin{array}{l}2,376 \\
\text { cycles }\end{array}$ & $\begin{array}{l}2,798 \\
\text { cycles }\end{array}$ & 2,584 cycles \\
\hline $\begin{array}{l}\text { Cooler MTTF } \\
20 \text { mins. dwell }\end{array}$ & 3,847 cycles & $\begin{array}{l}4,538 \\
\text { cycles }\end{array}$ & $\begin{array}{l}4,612 \\
\text { cycles }\end{array}$ & 4,996 cycles \\
\hline $\begin{array}{l}\text { Cooler MTTF } \\
1 \text { min. dwell }\end{array}$ & 14,803 cycles & $\begin{array}{l}17,948 \\
\text { cycles }\end{array}$ & $\begin{array}{l}18,275 \\
\text { cycles }\end{array}$ & 19,895 cycles \\
\hline
\end{tabular}




\begin{tabular}{|c|c|c|c|c|}
\hline $\begin{array}{c}\text { Stresses } \\
\text { GaN on SiC }\end{array}$ & $-291 \mathrm{MPa}$ & $-290 \mathrm{MPa}$ & $-288 \mathrm{MPa}$ & $-288 \mathrm{MPa}$ \\
\hline $\begin{array}{c}\text { Critical Flaw Size } \\
\text { GaN }\end{array}$ & \multicolumn{3}{|c|}{$21 \mu \mathrm{m}$} \\
\hline
\end{tabular}

Table 8.2: Reliability Comparison

Discrete Diamond Mini-contact

\begin{tabular}{|c|c|c|c|}
\hline \multirow{2}{*}{ Parameter } & \multicolumn{3}{|c|}{$\begin{array}{l}\text { Discrete Diamond } \\
\text { Mini-contact }\end{array}$} \\
\hline & T-shaped & Lofted & Taper \\
\hline Hot-Spot Temperature & $136^{\circ} \mathrm{C}$ & $136^{\circ} \mathrm{C}$ & $136^{\circ} \mathrm{C}$ \\
\hline Maximum Deformation & $0.7 \mu \mathrm{m}$ & $0.66 \mu \mathrm{m}$ & $0.64 \mu \mathrm{m}$ \\
\hline $\begin{array}{c}\text { Maximum Deformation } \\
\text { Location }\end{array}$ & $\begin{array}{l}\text { TE Cooler Header } \\
\text { Edge }\end{array}$ & $\begin{array}{l}\text { TE Cooler } \\
\text { Header Edge }\end{array}$ & $\begin{array}{c}\text { TE Cooler } \\
\text { Header Edge }\end{array}$ \\
\hline $\begin{array}{c}\text { MTTF solder } \\
\text { Chip/Mini-contact } \\
596 \text { mins. dwell }\end{array}$ & 363 cycles & 368 cycles & 288 cycles \\
\hline $\begin{array}{c}\text { MTTF solder } \\
\text { Chip/Mini-contact } \\
20 \text { mins. dwell }\end{array}$ & 606 cycles & 617 cycles & 467 cycles \\
\hline $\begin{array}{c}\text { MTTF solder } \\
\text { Chip/Mini-contact } \\
1 \text { min. dwell } \\
\end{array}$ & 2,102 cycles & 1,849 cycles & 1,320 cycles \\
\hline $\begin{array}{c}\text { MTTF solder } \\
\text { Mini-contact/Cooler } \\
596 \text { mins. dwell }\end{array}$ & 914 cycles & 1,055 cycles & 1,957 cycles \\
\hline $\begin{array}{c}\text { MTTF solder } \\
\text { Mini-contact/Cooler } \\
20 \text { mins. dwell }\end{array}$ & 1,402 cycles & 1,635 cycles & 3,684 cycles \\
\hline $\begin{array}{c}\text { MTTF solder } \\
\text { Mini-contact/Cooler } \\
1 \text { min. dwell }\end{array}$ & 4,797 cycles & 5,705 cycles & 14,187 cycles \\
\hline $\begin{array}{l}\text { MTTF - Cooler } \\
596 \text { mins. dwell }\end{array}$ & 1,711 cycles & 1,815 cycles & 1,779 cycles \\
\hline $\begin{array}{l}\text { MTTF - Cooler } \\
20 \text { mins. dwell }\end{array}$ & 2,718 cycles & 3,360 cycles & 3,287 cycles \\
\hline $\begin{array}{l}\text { MTTF - Cooler } \\
1 \text { min. dwell }\end{array}$ & 9,964 cycles & 12,433 cycles & 12,128 cycles \\
\hline
\end{tabular}




\begin{tabular}{|c|c|c|c|}
\hline $\begin{array}{c}\text { Stresses } \\
\text { GaN on SiC }\end{array}$ & $-286 \mathrm{MPa}$ & $-285 \mathrm{MPa}$ & $-285 \mathrm{MPa}$ \\
\hline $\begin{array}{c}\text { Critical Flaw Size } \\
\text { GaN }\end{array}$ & $21 \mu \mathrm{m}$ \\
\hline
\end{tabular}

Table 8.3: Reliability Comparison

Silver-Diamond Composite as Mini-contact Material

\begin{tabular}{|c|c|c|c|}
\hline \multirow[t]{2}{*}{ Parameter } & \multicolumn{3}{|c|}{$\begin{array}{l}\text { Discrete Silver-Diamond } \\
\text { Composite Mini-contact }\end{array}$} \\
\hline & T-shaped & Lofted & Taper \\
\hline $\begin{array}{c}\text { Hot-Spot } \\
\text { Temperature }\end{array}$ & $148^{\circ} \mathrm{C}$ & $147^{\circ} \mathrm{C}$ & $147^{\circ} \mathrm{C}$ \\
\hline $\begin{array}{c}\text { Maximum } \\
\text { Deformation }\end{array}$ & $1.54 \mu \mathrm{m}$ & $1.53 \mu \mathrm{m}$ & $1.53 \mu \mathrm{m}$ \\
\hline $\begin{array}{c}\text { Maximum } \\
\text { Deformation } \\
\text { Location } \\
\end{array}$ & $\begin{array}{c}\text { TE Cooler } \\
\text { Header } \\
\text { Edge } \\
\end{array}$ & $\begin{array}{c}\text { TE Cooler } \\
\text { Header } \\
\text { Edge } \\
\end{array}$ & $\begin{array}{c}\text { TE Cooler } \\
\text { Header } \\
\text { Edge }\end{array}$ \\
\hline $\begin{array}{l}\text { MTTF solder } \\
\text { TE Cooler } \\
596 \text { mins. dwell }\end{array}$ & $\begin{array}{l}2,608 \\
\text { cycles }\end{array}$ & $\begin{array}{l}2,612 \\
\text { cycles }\end{array}$ & $\begin{array}{l}2,545 \\
\text { cycles }\end{array}$ \\
\hline $\begin{array}{l}\text { MTTF solder } \\
\text { TE Cooler } \\
20 \text { mins. dwell } \\
\end{array}$ & $\begin{array}{l}5,820 \\
\text { cycles }\end{array}$ & $\begin{array}{l}5,022 \\
\text { cycles }\end{array}$ & $\begin{array}{l}4,885 \\
\text { cycles }\end{array}$ \\
\hline $\begin{array}{l}\text { MTTF solder } \\
\text { TE Cooler } \\
1 \text { min. dwell } \\
\end{array}$ & $\begin{array}{l}23,342 \\
\text { cycles }\end{array}$ & $\begin{array}{l}20,142 \\
\text { cycles }\end{array}$ & $\begin{array}{l}19,511 \\
\text { cycles }\end{array}$ \\
\hline $\begin{array}{l}\text { Von-Mises Stress } \\
\text { in Mini-contact }\end{array}$ & $2.83 \mathrm{GPa}$ & $2.77 \mathrm{GPa}$ & $2.87 \mathrm{GPa}$ \\
\hline $\begin{array}{l}\text { Plastic Strain in } \\
\text { Metallized Copper } \\
\text { - TE Cooler }\end{array}$ & $9.06 \mathrm{e}-3$ & $9.09 \mathrm{e}-3$ & $9.32 \mathrm{e}-3$ \\
\hline $\begin{array}{l}\text { Principal Stress - } \\
\text { Thermoelectric } \\
\text { Element (Max) }\end{array}$ & $74 \mathrm{MPa}$ & $72 \mathrm{MPa}$ & $73 \mathrm{MPa}$ \\
\hline $\begin{array}{l}\text { Principal Stress - } \\
\text { Thermoelectric } \\
\text { Element (Min) }\end{array}$ & $-59 \mathrm{MPa}$ & $-60 \mathrm{MPa}$ & $-61 \mathrm{MPa}$ \\
\hline
\end{tabular}




\begin{tabular}{|c|c|c|c|}
\hline $\begin{array}{c}\text { Principal Stress - } \\
\text { TE Cooler Header } \\
\text { (Max) }\end{array}$ & $\begin{array}{c}135.82 \\
\mathrm{MPa}\end{array}$ & $\begin{array}{c}132.97 \\
\mathrm{MPa}\end{array}$ & $137 \mathrm{MPa}$ \\
\hline $\begin{array}{c}\text { Principal Stress - } \\
\text { TE Cooler Header } \\
\text { (Min) }\end{array}$ & $-152 \mathrm{MPa}$ & $\begin{array}{c}-186.52 \\
\mathrm{MPa}\end{array}$ & $\begin{array}{c}-237.72 \\
\mathrm{MPa}\end{array}$ \\
\hline
\end{tabular}

Based on thermo-mechanical stress analysis data presented above for integrated and discrete mini-contact options, following conclusions can be drawn. It should be noted that MTTF predictions made using Engelmaier's failure model will have an accuracy of $+/-15 \%$.

1. An integrated SiC Mini-contact proves to be the most reliable option for Minicontact/TE Cooler integration. Mean Time to Failure (MTTF) of 3,867 cycles, 7876 cycles and 36,191 cycles was determined based on Engelmaier's failure model for dwell times of 596 minutes, 20 minutes and 1 minute each. The failure site shifts in TE Cooler.

2. For an integrated SiC Mini-contact option, the failure site in TE Cooler is solder joint interconnecting TE Elements with metallized Copper deposited on AIN headers. MTTF = 2,039 cycles, 3,847 cycles and 14,803 cycles.

3. For a discrete Copper Mini-contact option, the failure site shifts to solder joint interconnecting the Mini-contact with TE Cooler. MTTF = 104 cycles, 160 cycles and 404 cycles (T-shaped); 110 cycles, 169 cycles and 426 cycles (Lofted) and 308 cycles, 512 cycles and 1,519 cycles (Taper). The solder joint at chip level integration has comparatively very high reliability life. $\mathrm{MTTF}=39,300$ cycles, 92,447 cycles and 569,205 cycles (T-shaped); 38,594, 90,783 cycles and 559,262 cycles (Lofted) and 15,973 cycles, 35,456 cycles and 193,797 cycles (Taper). 
4. For a discrete Diamond Mini-contact, the failure site shifts to chip level integration, where solder joint interconnects Mini-contact with SiC chip. MTTF = 363 cycles, 606 cycles and 2,102 cycles (T-shaped); 368 cycles, 617 cycles and 1,849 cycles (Lofted) and 288 cycles, 467 cycles and 1,320 cycles (Taper).

5. For discrete Mini-contact, the solder joint in TE Cooler has a comparatively higher reliability than an integrated SiC Mini-contact. MTTF for Copper Minicontact is higher than Diamond Mini-contact. For Copper, reliability values, MTTF $=2,376$ cycles, 4,538 cycles and 17,948 cycles (T-shaped); 2,798 cycles, 4,612 cycles and 18,275 cycles (Lofted) and 2,584 cycles, 4,996 cycles and 19,895 cycles (Taper). For Diamond, reliability values, MTTF $=1,711$ cycles, 2,718 cycles and 9,964 cycles (T-shaped); 1,815 cycles, 3,360 cycles and 12,433 cycles (Lofted) and 1,779 cycles, 3,287 cycles and 12,128 cycles (Taper).

6. The maximum deformation site shifts with change in integration type and also on material selection of Mini-contact. For an integrated SiC Mini-contact and Diamond Mini-contact options, the deformation site is TE Cooler header edge. For Copper Mini-contact, the deformation site is solder joint at chip level.

7. Maximum deformation in the structure is in range of $1 \mu \mathrm{m}-2 \mu \mathrm{m}$.

8. The stress concentrations for corners at micro-fins have a corresponding critical crack size of $5 \mu \mathrm{m}$. In order to predict if the structure will fail at this location due to cyclic fatigue, further study using fracture mechanics approach should be used.

9. The critical crack size calculated based on linear elastic fracture mechanics approach for AlN headers and thermo-electric elements are relatively large in size and will not be of a concern. A flaw size of $21 \mu \mathrm{m}$ was determined in GaN. 
10. The critical flaw size for diamond Mini-contact is relatively large in size and will not be of a concern. The Mini-contact structure will not fail by brittle fracture (SiC or Diamond Mini-contact) and ductile fracture (Copper Mini-contact) due to compressive stresses.

11. With use of Silver-Diamond composite as a Mini-contact material, the MTTF of TE Cooler obtained was highest for a lofted Mini-contact. 2,612 cycles, 5,022 cycles and 20,142 cycles (Lofted), MTTF $=2,608$ cycles, 5,820 cycles and 23,342 cycles (T-shaped) and 2,545 cycles, 4,885 cycles and 19,511 cycles (Taper).

12. For a Mini-contact manufactured out of Silver-Diamond composite, there are stress concentrations at chip level, which are approximately in range of $3 \mathrm{GPa}$.

13. There is high stress concentration in $\mathrm{GaN}$ device on $\mathrm{SiC}$ substrate. These stresses are compressive in nature ( -290 MPa) and will in turn affect the electrical performance of the device due to reverse piezoelectric effect seen in HEMTs.

\section{$\underline{8.2 \text { Contributions }}$}

Following contributions can be drawn from this work.

1. First structural analysis of an integrated thermo-electric hot-spot/manifold micro-channel cooling system.

2. Identified that the best solution was $\mathrm{SiC}$ integrated $\mathrm{SiC}$ Mini-contact for the conditions investigated, then Copper discrete and last diamond Mini-contact.

3. Determined the best shape to increase the solder joint life in a discrete Minicontact was tapered at the top and lofted Mini-contact at the bottom for maximum strain shedding. 
4. First thermal and structural evaluation of Silver-diamond composite as a Minicontact material.

5. Determined that for the micro-machining approach used in this study, there should be no cracking of the $\mathrm{SiC}$ at the corners of the micro-fins, but that fatigue might occur over time. 


\section{Appendix}

ANSYS Command language used for performing combined thermal and structural analysis is presented below:

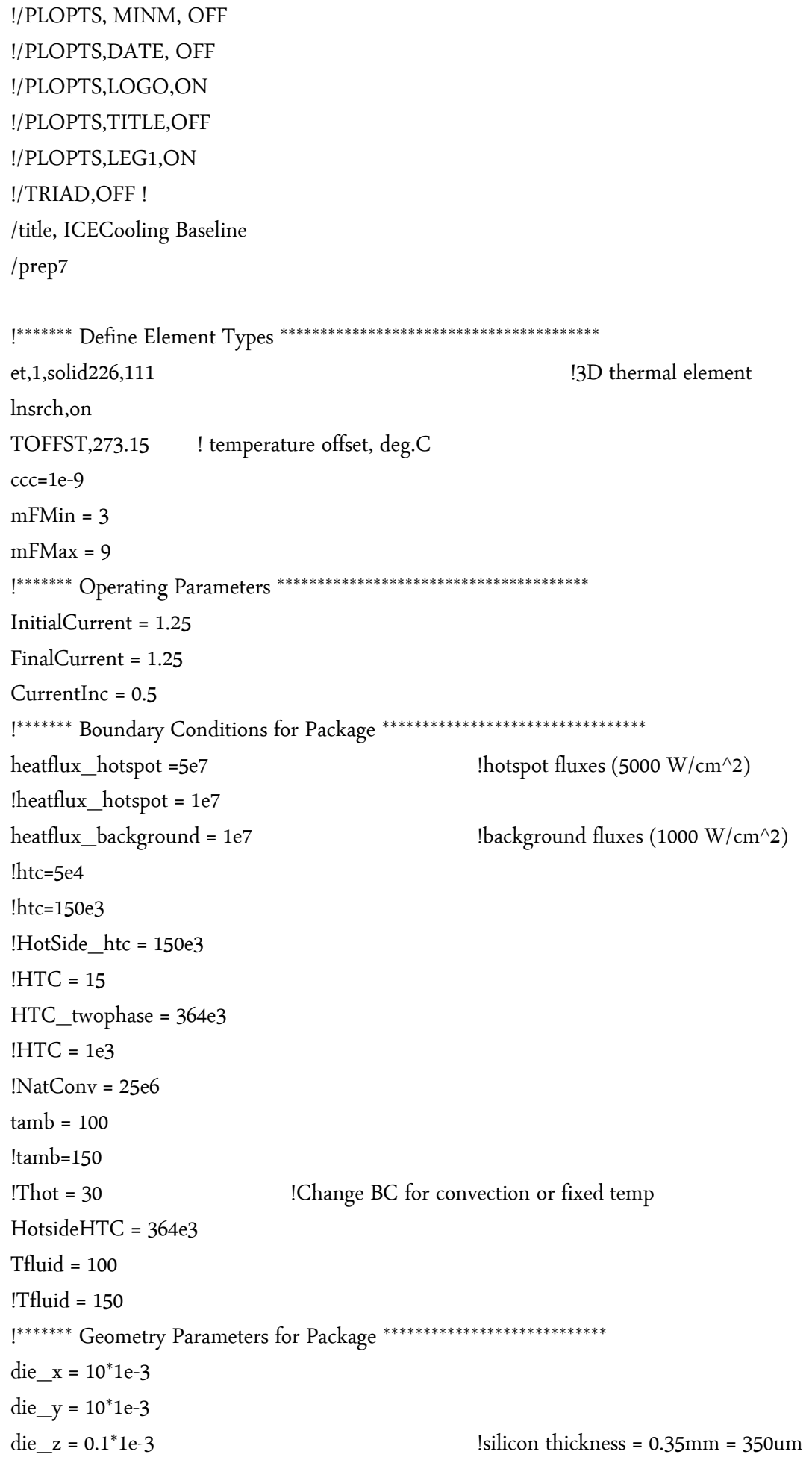




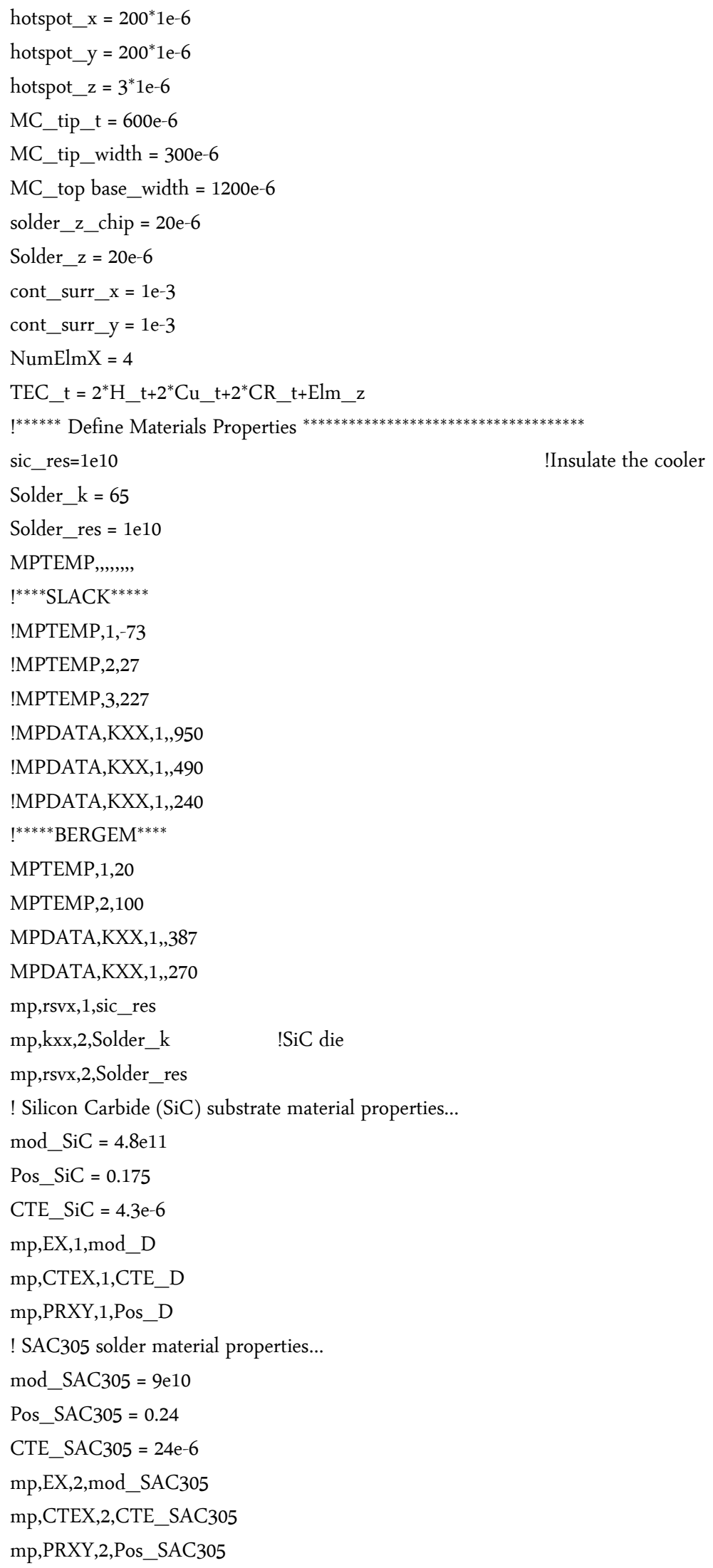




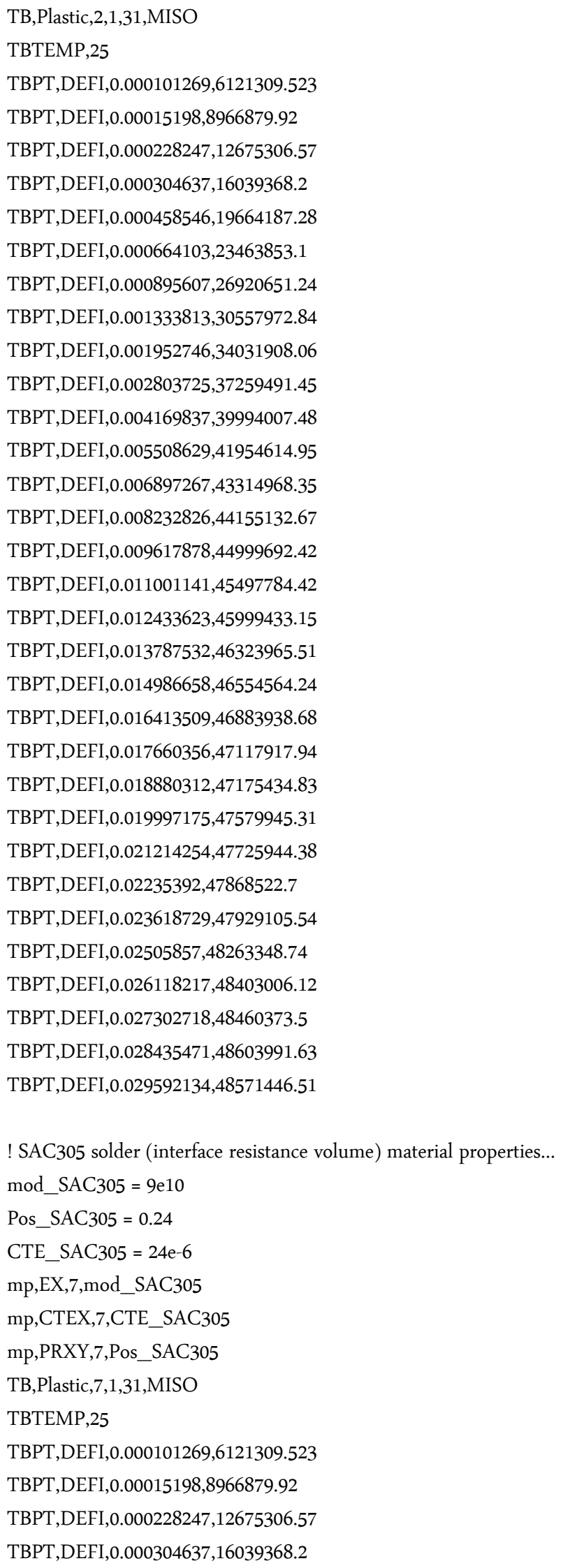


TBPT,DEFI,0.000458546,19664187.28 TBPT,DEFI,0.000664103,23463853.1 TBPT,DEFI,0.000895607,26920651.24 TBPT,DEFI,0.001333813,30557972.84 TBPT,DEFI,0.001952746,34031908.06 TBPT,DEFI,0.002803725,37259491.45 TBPT,DEFI,0.004169837,39994007.48 TBPT,DEFI,0.005508629,41954614.95 TBPT,DEFI,0.006897267,43314968.35 TBPT,DEFI,0.008232826,44155132.67 TBPT,DEFI,0.009617878,44999692.42 TBPT,DEFI,0.011001141,45497784.42 TBPT,DEFI,0.012433623,45999433.15 TBPT,DEFI,0.013787532,46323965.51 TBPT,DEFI,0.014986658,46554564.24 TBPT,DEFI,0.016413509,46883938.68 TBPT,DEFI,0.017660356,47117917.94 TBPT,DEFI,0.018880312,47175434.83 TBPT,DEFI,0.019997175,47579945.31 TBPT,DEFI,0.021214254,47725944.38 TBPT,DEFI,0.02235392,47868522.7 TBPT,DEFI,0.023618729,47929105.54 TBPT,DEFI,0.02505857,48263348.74 TBPT,DEFI,0.026118217,48403006.12 TBPT,DEFI,0.027302718,48460373.5 TBPT,DEFI,0.028435471,48603991.63 TBPT,DEFI,0.029592134,48571446.51

! Copper material properties...(TEC)

mod_Copper $=1.1 \mathrm{e} 11$

Pos_Copper $=0.343$

CTE_Copper $=16.4 \mathrm{e}-6$

mp,EX,5,mod_Copper

mp,CTEX,5,CTE_Copper

mp,PRXY,5,Pos_Copper

TB,Plastic,5,4,25,MISO

TBTEMP,20

TBPT,DEFI,0.006106519742883368, 41911764.70588231 TBPT,DEFI,0.01157024793388428, 51470588.23529406 TBPT,DEFI,0.018319559228650126, 61764705.88235292 TBPT,DEFI,0.027318640955004546, 75367647.0588234 TBPT,DEFI,0.03374655647382914, 84926470.58823527 TBPT,DEFI,0.04210284664830116, 95588235.29411758 TBPT,DEFI,0.05367309458218539, 109926470.58823518 TBPT,DEFI,0.06524334251606968, 122794117.64705876 TBPT,DEFI,0.0790633608815426, 137132352.94117644 TBPT,DEFI,0.08934802571166196, 147426470.58823514 
TBPT,DEFI,0.10188246097336996, 158823529.4117645 TBPT,DEFI,0.11955922865013768, 170955882.3529411 TBPT,DEFI,0.1337006427915517, 181249999.99999994 TBPT,DEFI,0.14816345270890705, 191176470.5882352 TBPT,DEFI,0.1597337006427915, 197426470.5882351 TBPT,DEFI,0.17612488521579409, 205514705.88235268 TBPT,DEFI,0.18865932047750222, 212132352.94117635 TBPT,DEFI,0.20601469237832842, 219117647.05882323 TBPT,DEFI,0.2243342516069788, 225735294.11764687 TBPT,DEFI,0.24201101928374624, 231617647.0588232 TBPT,DEFI,0.2580808080808079, 237132352.94117612 TBPT,DEFI,0.27704315886134057, 241176470.588235 TBPT,DEFI,0.29761248852157945, 244852941.17647022 TBPT,DEFI,0.33296602387511454, 250735294.117647 TBPT,DEFI,0.3493572084481174, 254779411.7647056

TBTEMP,75

TBPT,DEFI,0.0080149631060323, 31823085.745602816 TBPT,DEFI,0.016327707712264584, 45997418.85868464 TBPT,DEFI,0.022721501923701638, 56391661.67601814 TBPT,DEFI,0.030710554171924285, 66784468.070530474 TBPT,DEFI,0.03710396165414025, 76863559.72093621 TBPT,DEFI,0.04573189057551929, 87886093.88017578 TBPT,DEFI,0.05499908289927574, 99853506.97107042 TBPT,DEFI,0.06266714989403607, 108670844.81550787 TBPT,DEFI,0.06937728860828303, 116858742.17978263 TBPT,DEFI,0.08023857878116201, 127879265.34707287 TBPT,DEFI,0.09205779723455451, 139529228.99452612 TBPT,DEFI,0.10259771542475003, 148659132.44481358 TBPT,DEFI,0.11249798348334725, 156529005.7965183 TBPT,DEFI,0.12559186144928416, 166917215.6380035 TBPT,DEFI,0.13804570255440166, 175730244.2139782 TBPT,DEFI,0.15752254631358645, 187688464.19881836 TBPT,DEFI,0.1674208807260783, 193982581.71588436 TBPT,DEFI,0.17987201472664838, 200589552.12336433 TBPT,DEFI,0.19327720971552187, 204674451.34172913 TBPT,DEFI,0.2060470085942282, 210965983.29771706 TBPT,DEFI,0.21913431216340734, 215996623.3014297 TBPT,DEFI,0.2462649139361741, 225111587.9543788 TBPT,DEFI,0.26669117793300934, 230765922.74697065 TBPT,DEFI,0.3021163480386195, 239243115.66739509 TBPT,DEFI,0.349983370643495, 246763650.98903224

TBTEMP,125

TBPT,DEFI,0.0059353449334348385, 22290559.160197325 TBPT,DEFI,0.00906613381792245, 28578829.1919398 TBPT,DEFI,0.015018294876900635, 37726545.24178381 
TBPT,DEFI,0.02598427718953964, 53449782.778365545 TBPT,DEFI,0.032251460333696025, 61740613.1319526 TBPT,DEFI,0.043219311104728715, 76035280.76523514 TBPT,DEFI,0.050428197279311054, 84326879.8559899 TBPT,DEFI,0.06484783808686939, 99481508.13420002 TBPT,DEFI,0.07895320419260705, 114921594.14734763 TBPT,DEFI,0.08616470620894048, 121213195.37348348 TBPT,DEFI,0.09369048292709488, 127219338.8646818 TBPT,DEFI,0.11940308626619009, 148097471.60210148 TBPT,DEFI,0.12818484071659114, 153818926.09553033 TBPT,DEFI,0.136966595166992, 159540380.58895943 TBPT,DEFI,0.1466900526478192, 165262603.81955585 TBPT,DEFI,0.16927410925249906, 178138182.64127415 TBPT,DEFI,0.1821362031430673, 184148682.3097561 TBPT,DEFI,0.20347026108236052, 192737526.43868622 TBPT,DEFI,0.21068512632380276, 196457701.8388834 TBPT,DEFI,0.2417493681941401, 205625917.54653236 TBPT,DEFI,0.2593210983118741, 210783118.95887333 TBPT,DEFI,0.2863079902754747, 217376577.64619073 TBPT,DEFI,0.3054518412856699, 220535062.42252547 TBPT,DEFI,0.3349518097887996, 225702001.17232382 TBPT,DEFI,0.34970104665700724, 228856898.50854278

TBTEMP,175

TBPT,DEFI,0.007487445571167309, 18199513.38199513 TBPT,DEFI,0.015612590733512358, 28905109.489051044 TBPT,DEFI,0.023740103592359306, 41216545.0121654 TBPT,DEFI,0.03576602874158057, 57542579.07542572 TBPT,DEFI,0.04908668926125611, 71995133.81995124 TBPT,DEFI,0.05948363921717183, 83503649.63503642 TBPT,DEFI,0.07312551722893822, 95815085.15815073 TBPT,DEFI,0.081247505462614, 104379562.04379547 TBPT,DEFI,0.09293958540506768, 114282238.44282225 TBPT,DEFI,0.10885090051390275, 125790754.25790752 TBPT,DEFI,0.1218392942911455, 134890510.94890493 TBPT,DEFI,0.1377462686230604, 143454987.83454973 TBPT,DEFI,0.15170660181433177, 151751824.81751823 TBPT,DEFI,0.16696482730472684, 160316301.703163 TBPT,DEFI,0.18124479952375447, 165401459.85401458 TBPT,DEFI,0.19520276501852415, 172092457.4209244 TBPT,DEFI,0.20850803551093755, 176107055.96107036 TBPT,DEFI,0.22213925888844532, 181192214.11192194 TBPT,DEFI,0.23512015496009864, 185206812.65206793 TBPT,DEFI,0.2519943333130385, 189756690.99756685 TBPT,DEFI,0.27243663029433807, 194306569.34306568 TBPT,DEFI,0.29028353729347467, 198588807.7858878 TBPT,DEFI,0.31461674885955904, 202068126.5206812 
TBPT,DEFI,0.32726971896569995, 203673965.93673936

TBPT,DEFI,0.3503058274648282, 207420924.57420895

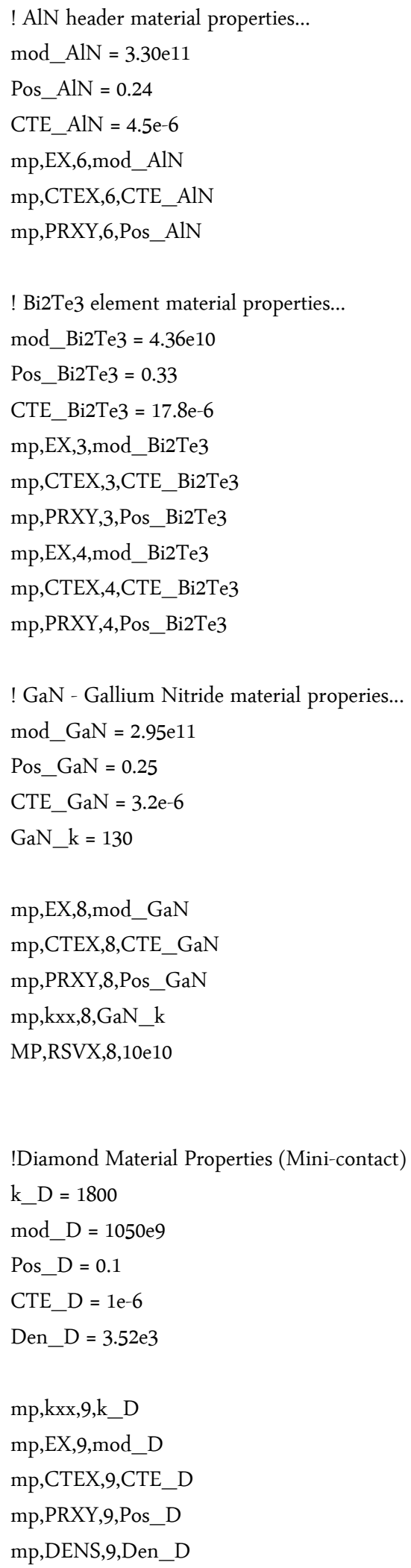




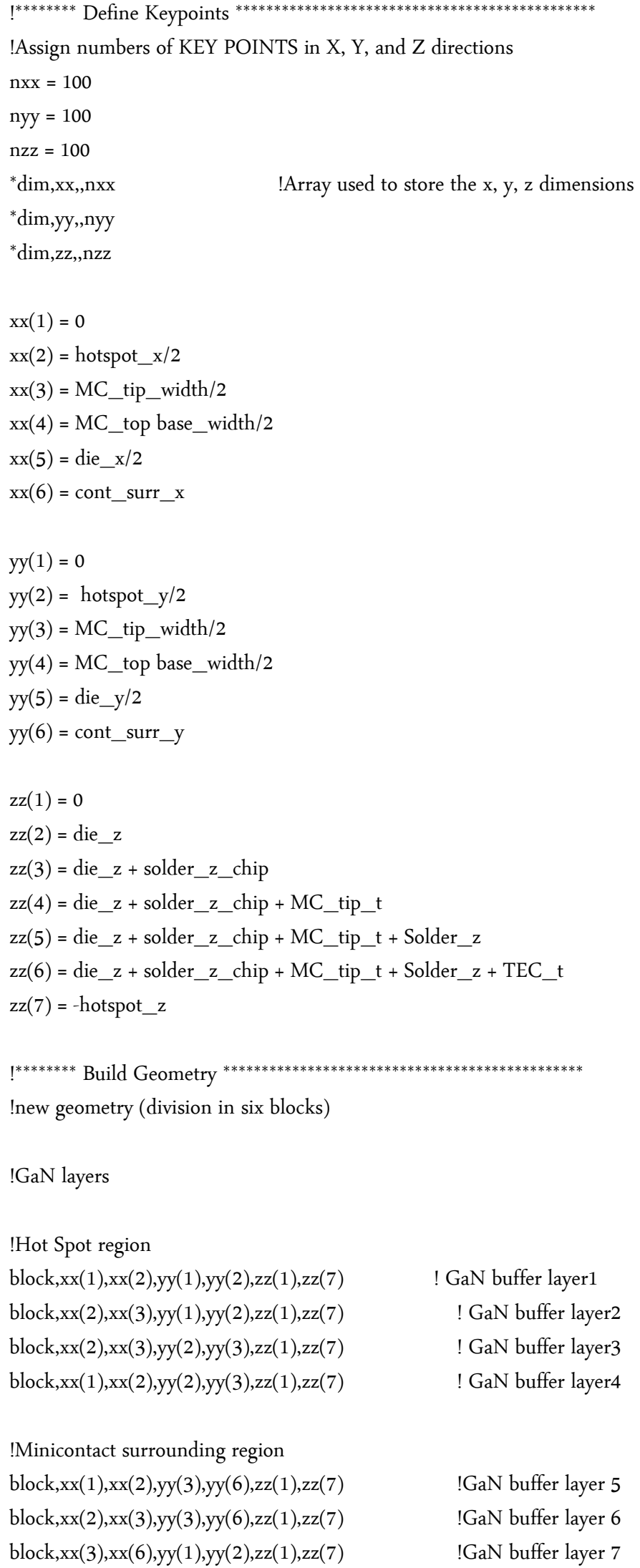




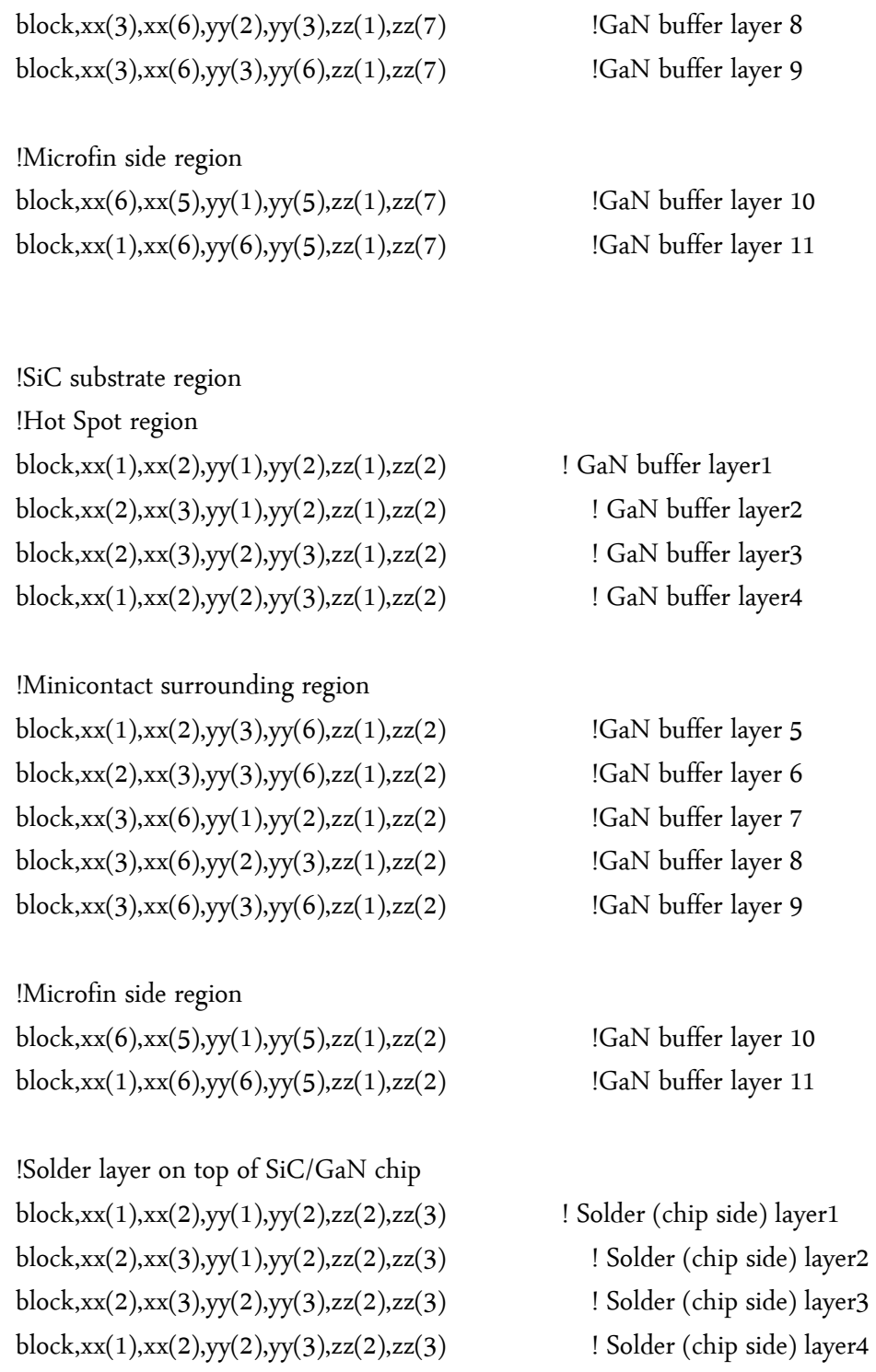

! Add T minicontact geometry by importing the CAD file

!Solder layer on top of minicontact

block,xx(1),xx(4),yy(1),yy(4),zz(4),zz(5)

$!^{* * * *}$ START BUILD COOLER ${ }^{* * * * * * * * * * * * * * * * * * * * * * *}$

ZOff $=$ die_z + Solder_z_chip + MC_tip_t + Solder_z

$!^{* * * * * * *}$ Geometry Parameters for Package *

Elm_x $=100^{*} 1 \mathrm{e}-6$

Elm_y $=$ Elm_x

Elm_s $=83.3^{*} 1 \mathrm{e}-6$

!Elm_z $=5^{*} 1 \mathrm{e}-6$

Elm_z $=50^{*} 1 \mathrm{e}-6$ 


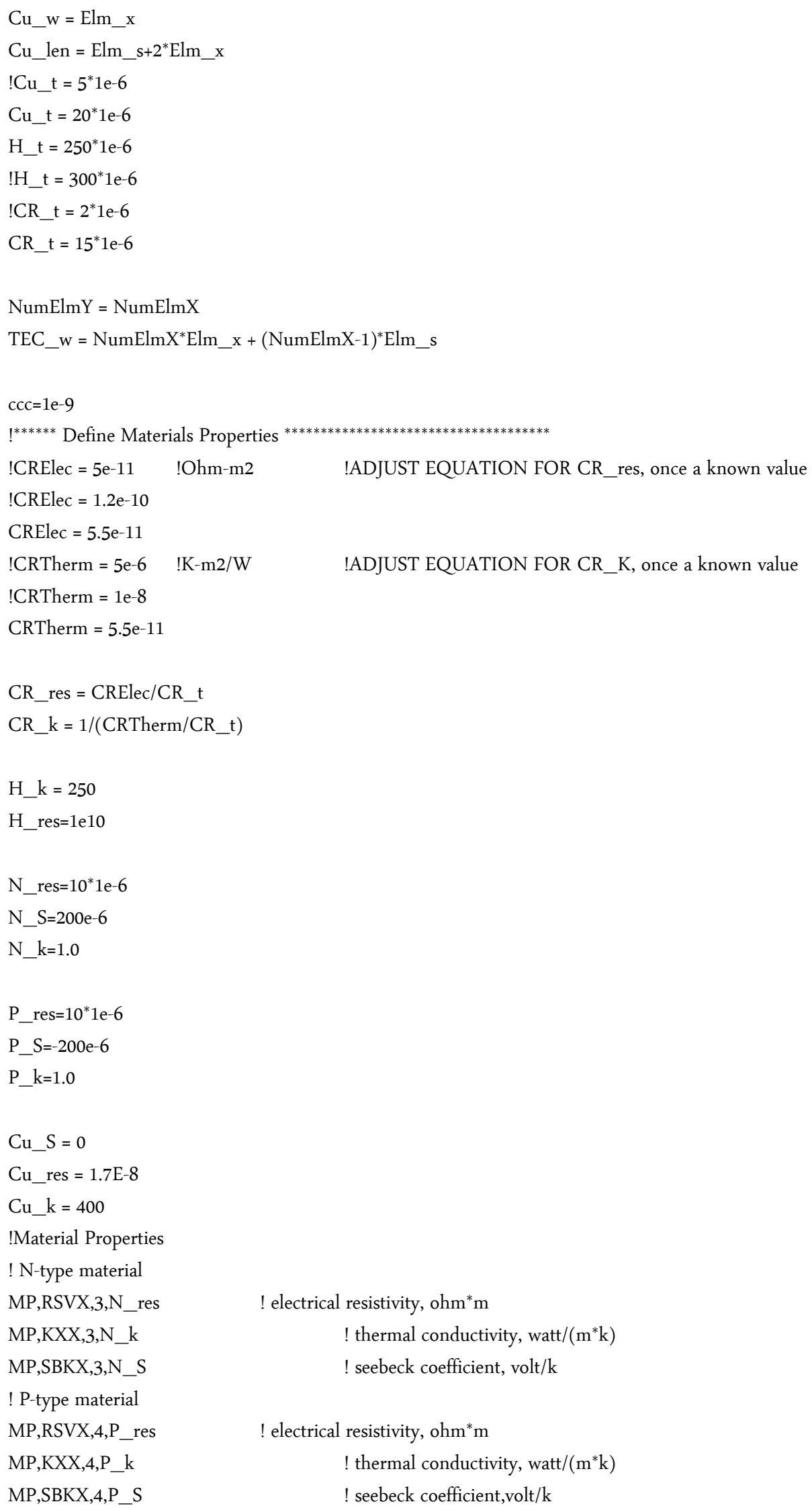




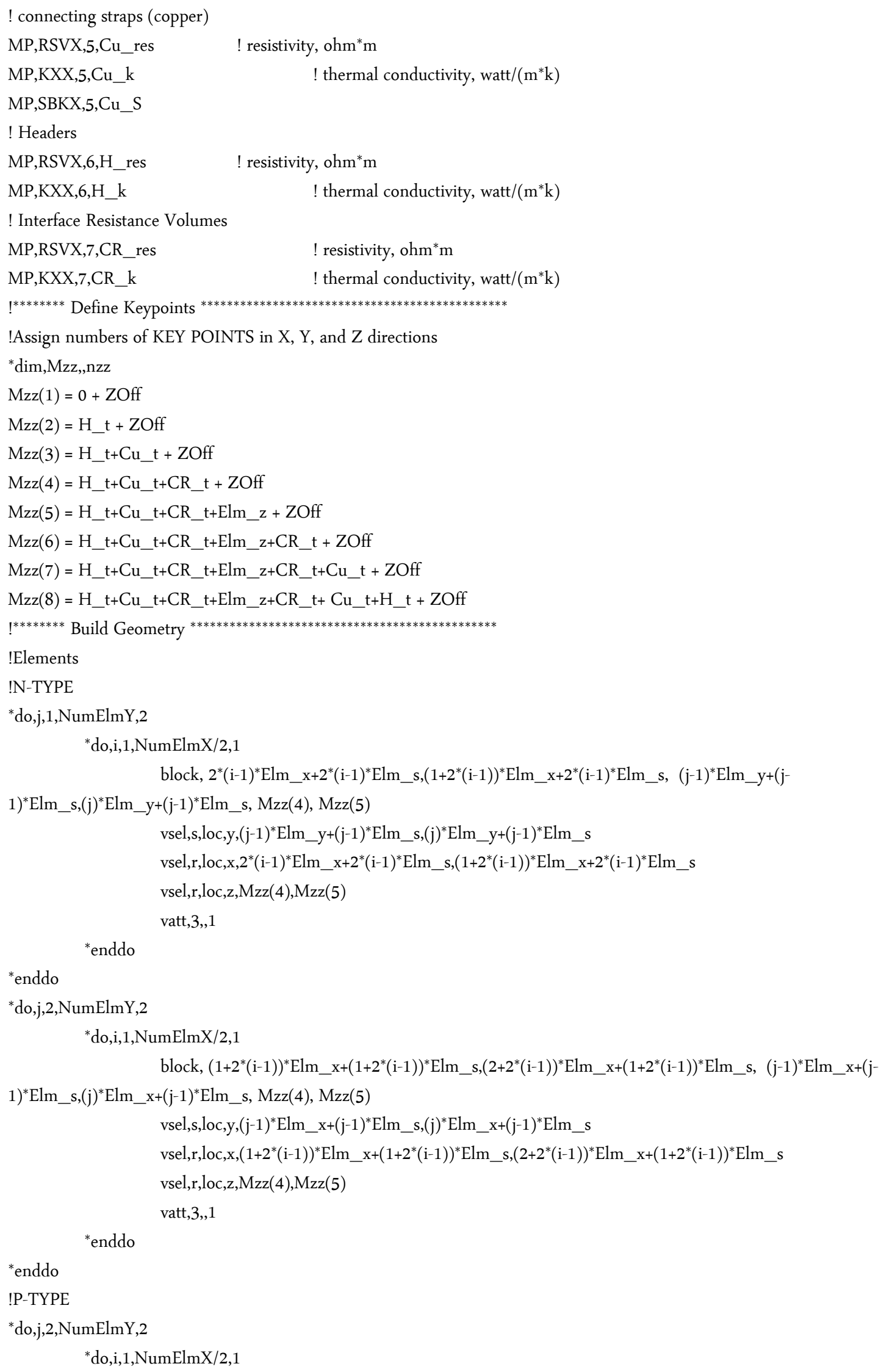


block, $2^{*}(\mathrm{i}-1)^{*} E l m \_\mathrm{x}+2^{*}(\mathrm{i}-1)^{*} \operatorname{Elm} \_\mathrm{s},\left(1+2^{*}(\mathrm{i}-1)\right)^{*} \mathrm{Elm} \_\mathrm{x}+2^{*}(\mathrm{i}-1)^{*} \mathrm{Elm} \_\mathrm{s}, \quad(\mathrm{j}-1)^{*} \mathrm{Elm} \_\mathrm{y}+(\mathrm{j}-$

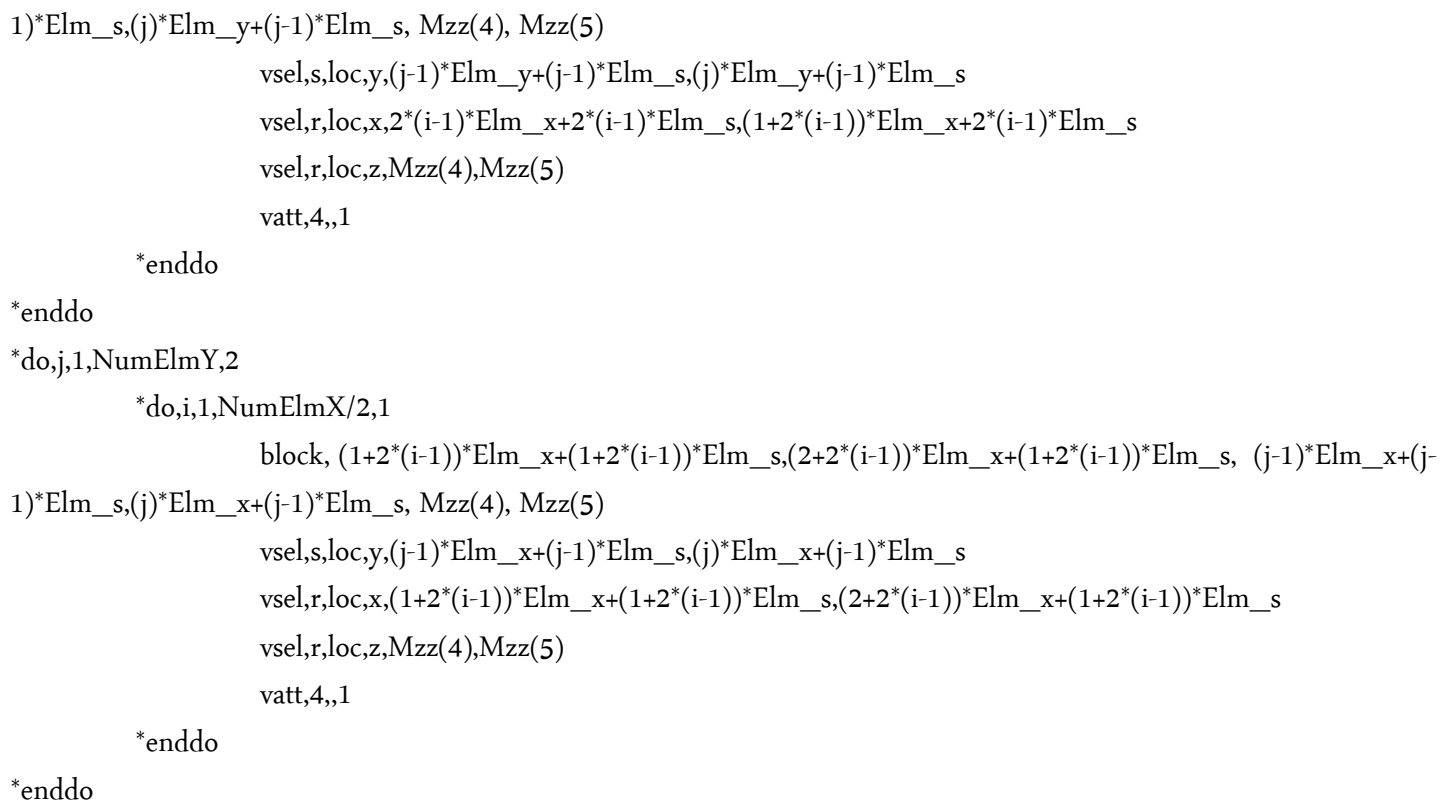




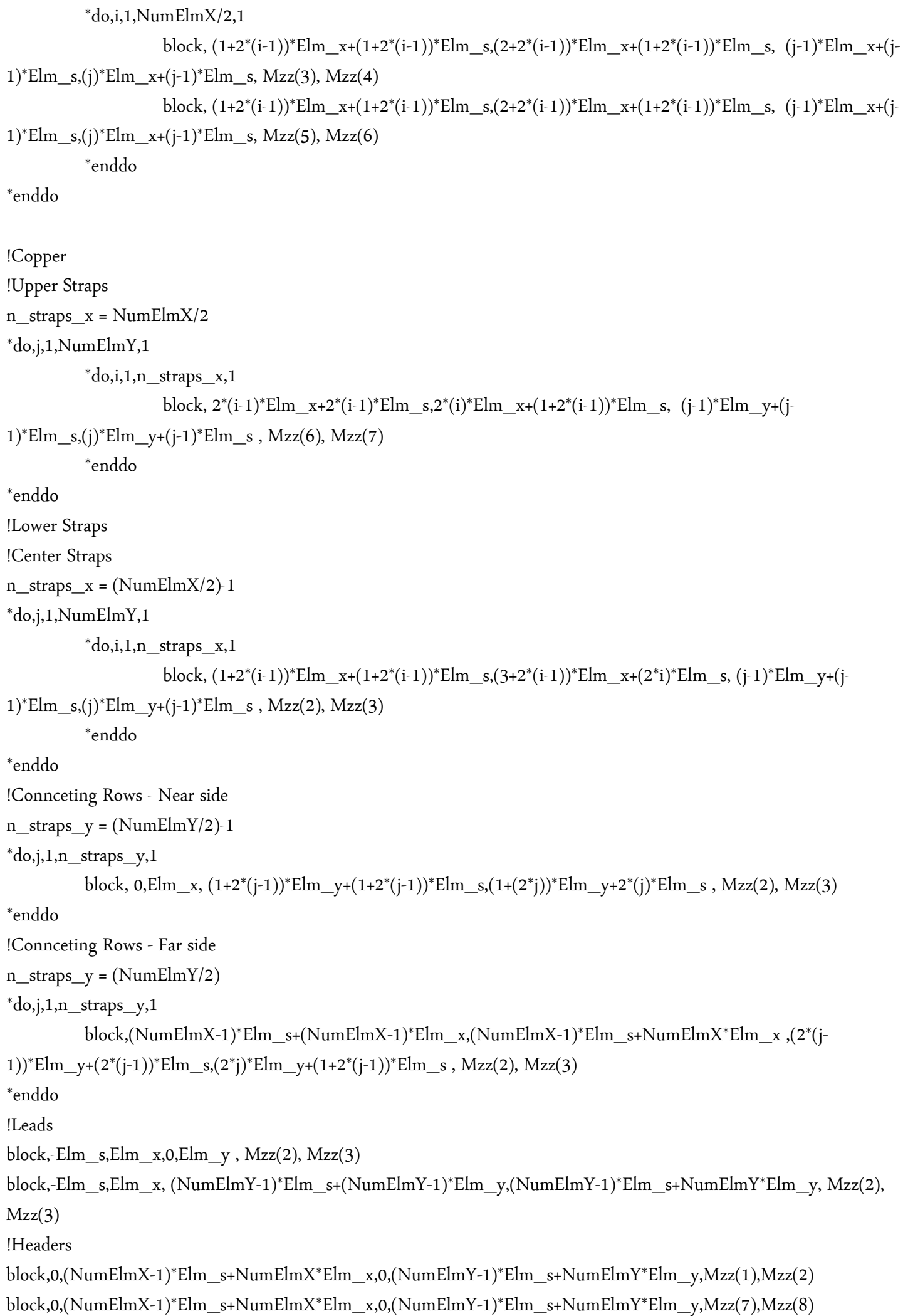




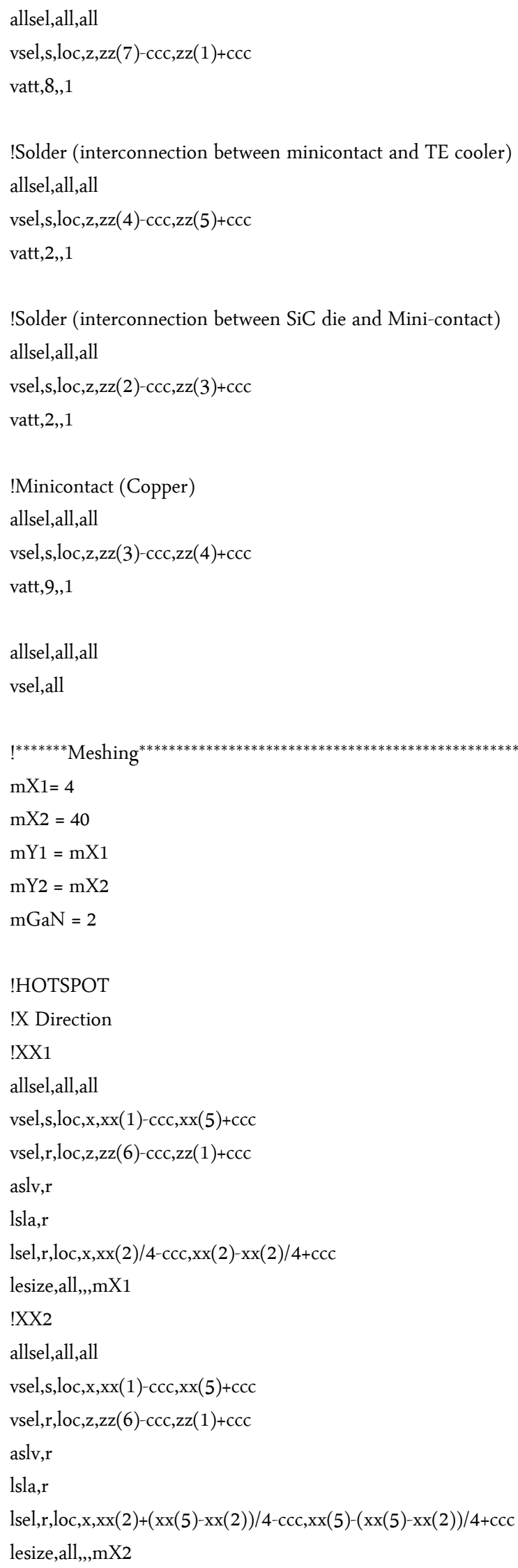




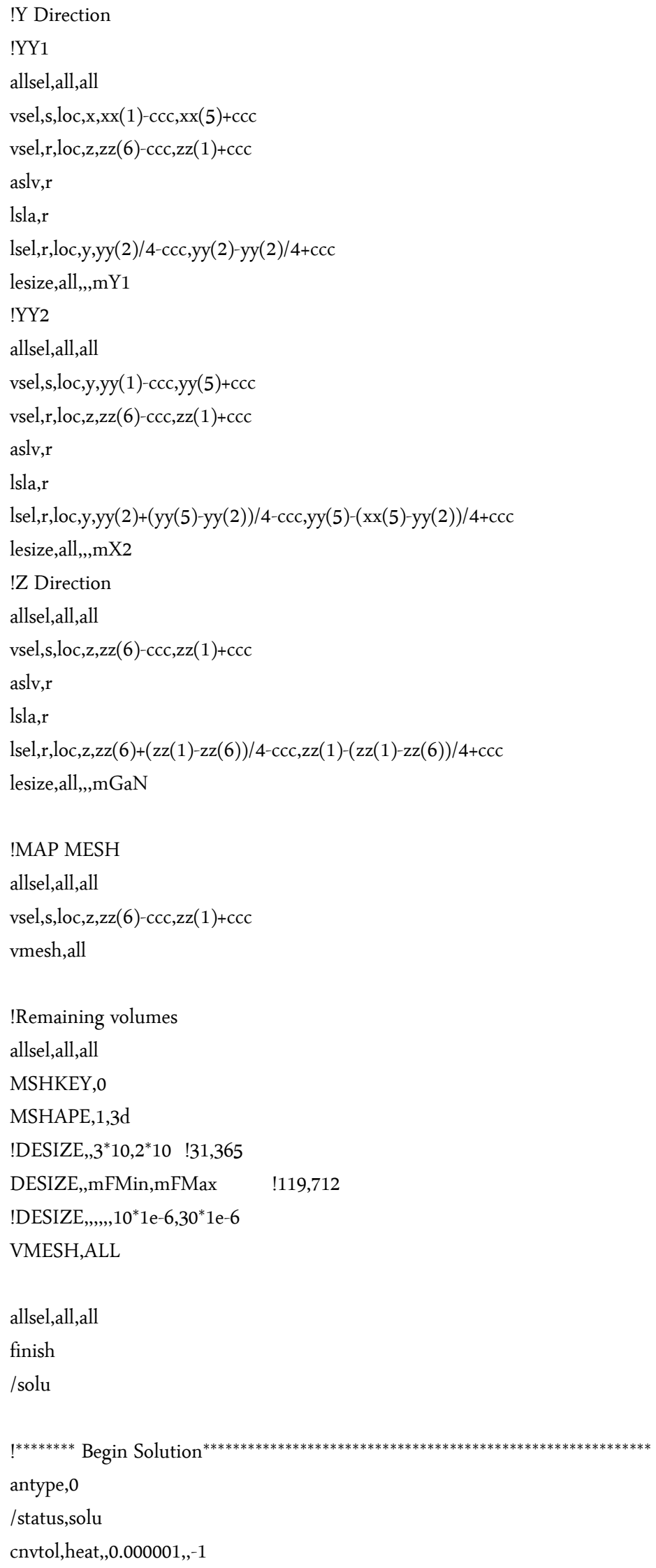




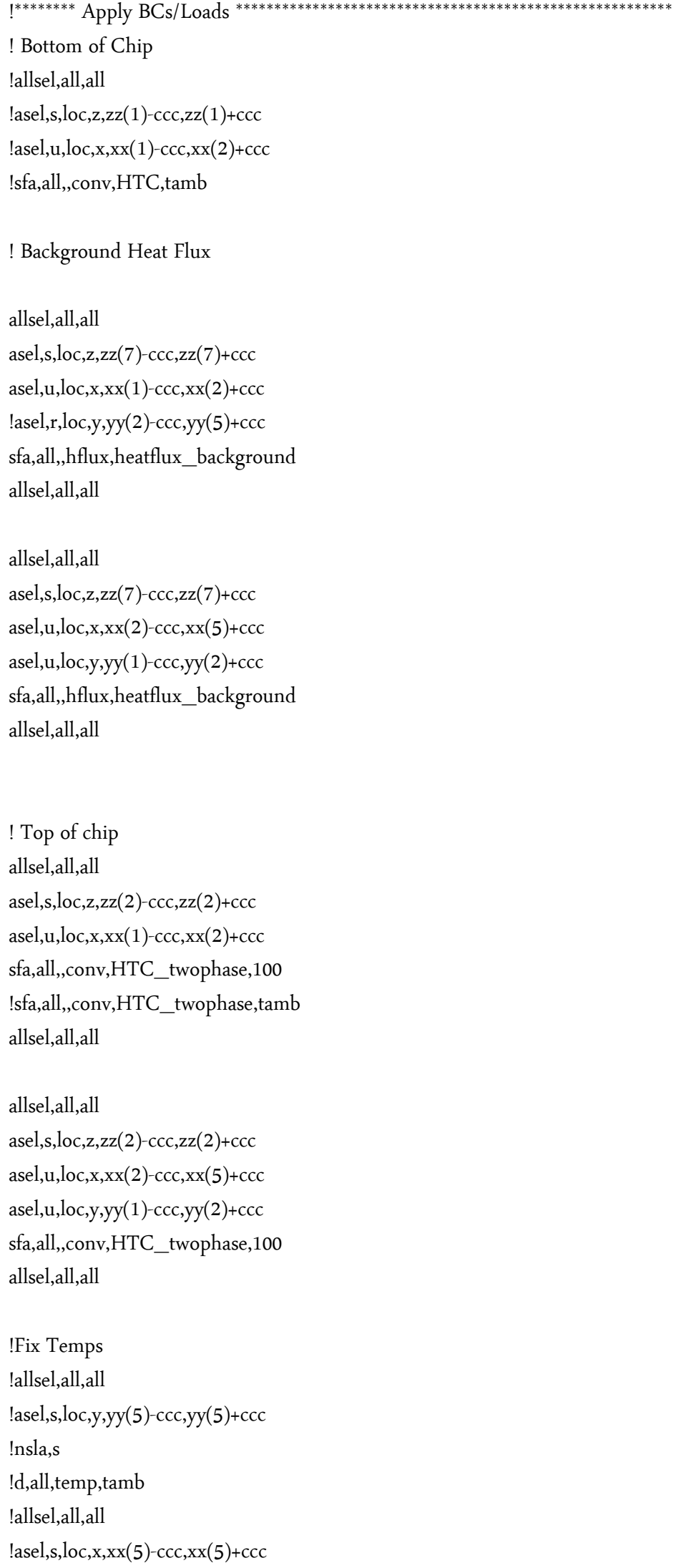




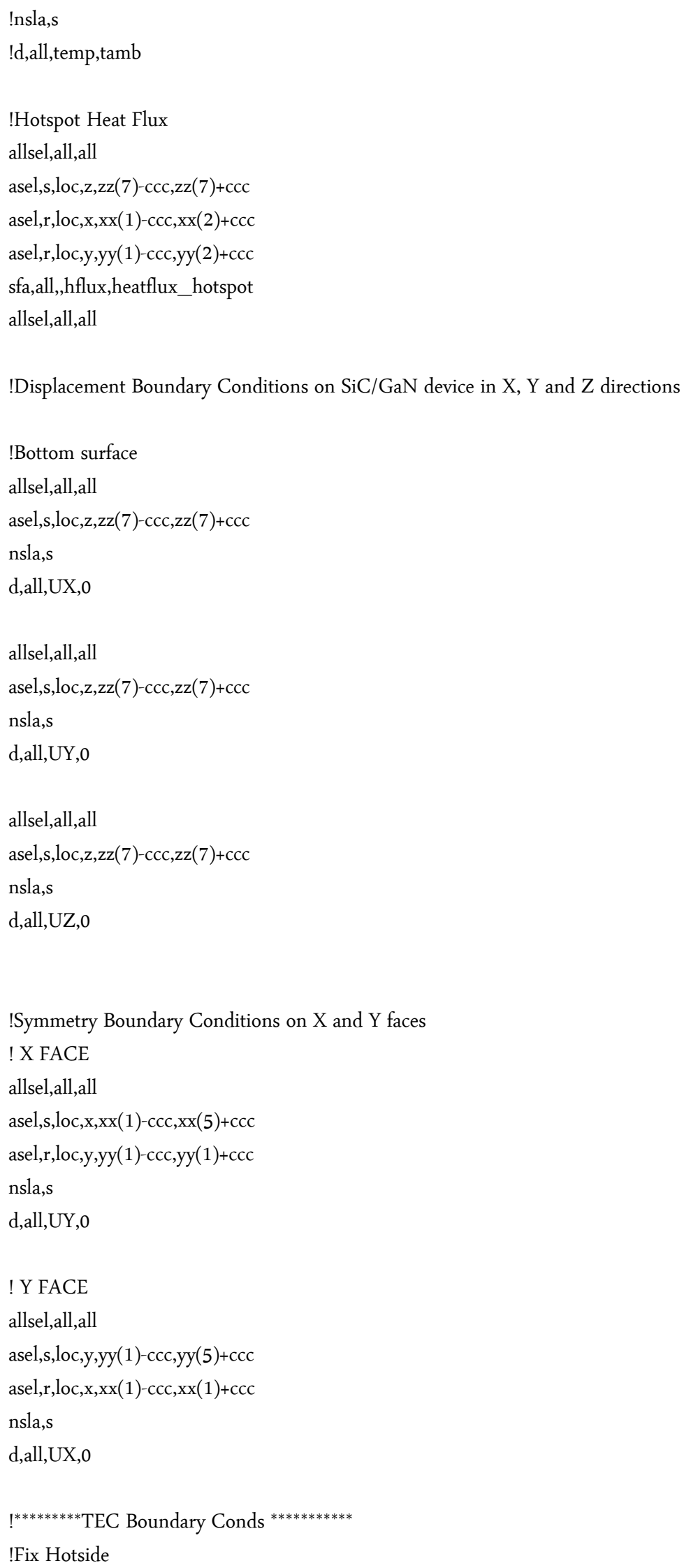

!Fix Hotside 


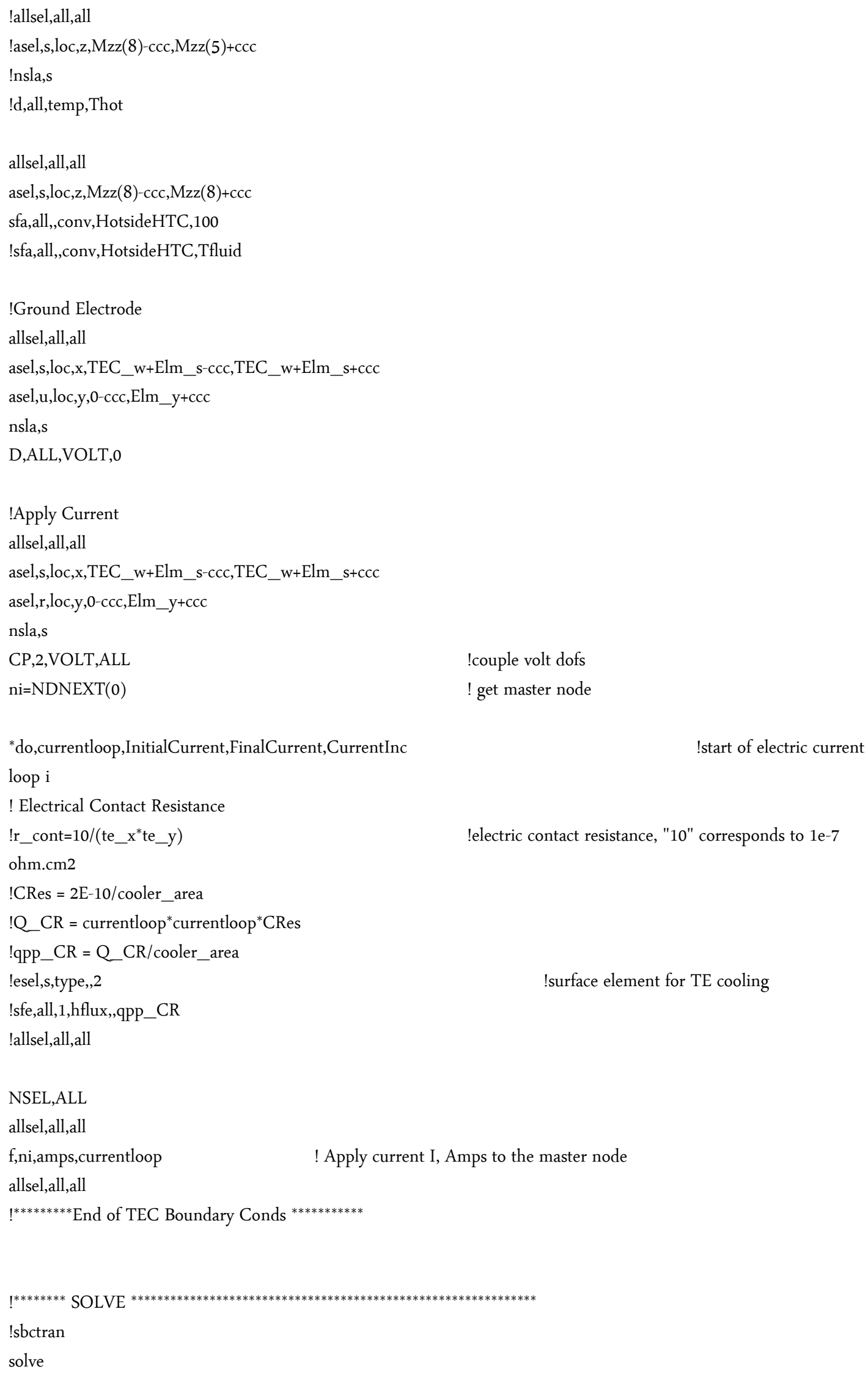




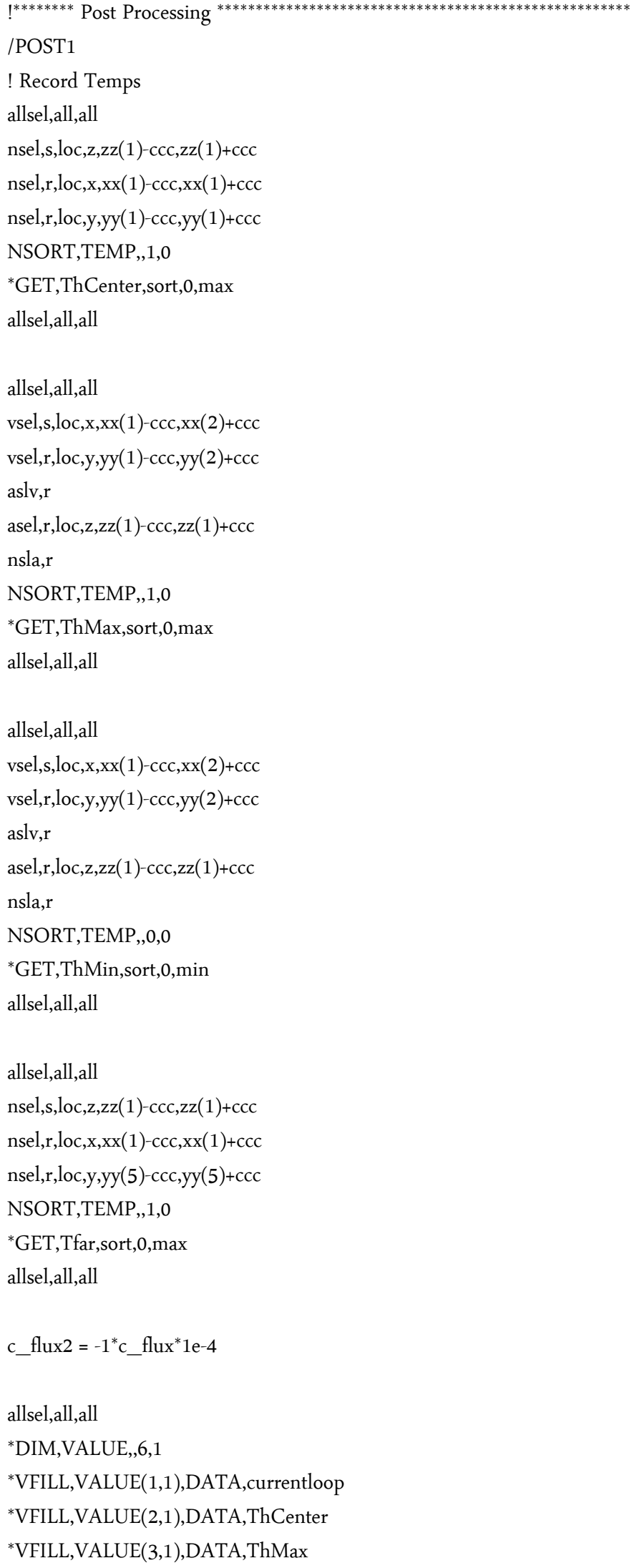


*VFILL,VALUE(4,1),DATA,ThMin

*VFILL,VALUE(5,1),DATA,c_flux2

*VFILL,VALUE(6,1),DATA,Tfar

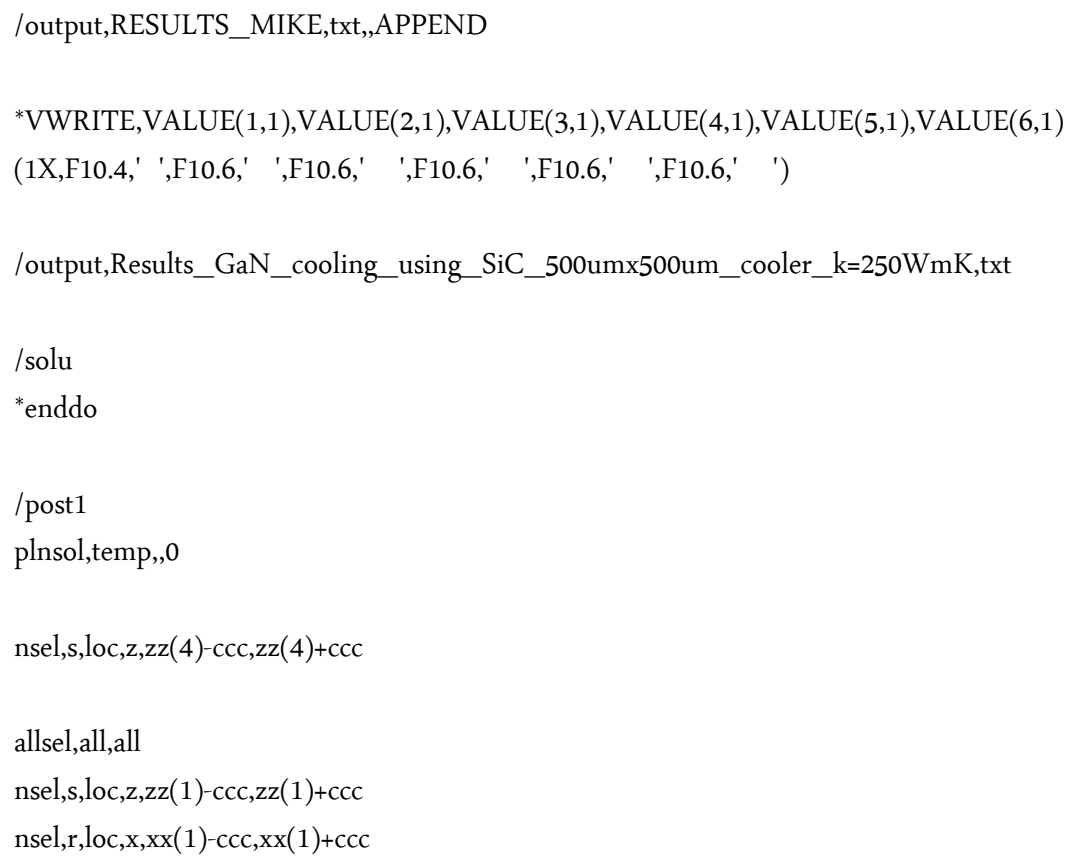




\section{References}

[1] "Power Electronics." Internet: http://en.wikipedia.org/wiki/Power_electronics [Sep. 2014]

[2] Lauren Boteler. "Microfabrication and Analysis of Manifold Microchannel Coolers for Power Electronics" Doctor of Philosophy Dissertation, University of Maryland at College Park, USA, 2011

[3] R.R. Tummula. Fundamentals of Microsystems Packaging. McGraw-Hill Publications, Georgia Institute of Technology, 2001, pp. 184-262

[4] C.A. Harper. Electronics Packaging and Interconnection handbook. McGraw-Hill Publications, 2000

[5] T.I. Bajenescu and M.I. Bazu. Reliability of Electronic Components - A Practical Guide to Electronic Systems Manufacturing. Springer, 1999

[6] J. Lau, C.P. Wong, J.L. Prince and Wataru Nakayama. Electronics Packaging Design, Materials, Process and Reliability. McGraw-Hill, 1998

[7] Y. Shabany. Heat Transfer - Thermal Management of Electronics. CRC Press, Taylor and Francis Group, 2010

[8] W.D. Callister and D.G. Rethwisch. Fundamentals of Materials Science and Engineering. John Wiley and Sons, 2005

[9] E. Colgan and J. Wakil. "Measured Thermal Resistance of Microbumps in 3D Chip Stacks." Internet: http://www.electronics-cooling.com/2013/03/measuredthermal-resistance-of-microbumps-in-3d-chip-stacks/ [Oct. 2014]

[10] "Thermoelectric Phenomena." Internet: http://en.wikipedia.org/thermoelectric phenomena [Sep. 2014 - Oct. 2014]

[11] "Thermoelectric Cooler." Internet: www.tetech.com [Sep. 2014 - Oct. 2014]

[12] G.J. Snyder, M. Soto, R. Alley, D. Koester and B. Conner. "Hot Spot Cooling using Embedded Thermoelectric Coolers," in Proc. IEEE SEMI-THERM Symposium, 2006, pp. 135-143

[13] R. Alley. "Reliability Testing of Thin-Film Superlattice Thermoelectric Device," in Proc. ICT'04 $23^{\text {rd }}$ International Conference on Thermoelectrics, 2004

[14] D. Koester, R. Venkatasubramanian, B. Conner, G.J. Snyder. "Embedded Thermoelectric Coolers for Semiconductor Hot Spot Cooling," in Proc. ITHERM, 2006, pp. 491-496

[15] M. Pecht. Product Reliability, Maintainability and Supportability Handbook. CRC Press, Technology and Engineering, 2009

[16] "Weibull Distribution." Internet: http://reliawiki.org/index.php/The_Weibull_Distribution

[17] "Bath Tub Curve." Internet: http://en.wikipedia.org/wiki/Bathtub_curve

[18] F.P. McCluskey. "Reliability of Power Electronics Under Thermal Loading." In Proc. CIPS 2012, pp. 4.1-4.8

[19] P. Hansen and F.P. McCluskey. "Failure Models in Power Device Interconnects," in Proc. EPE 2007, pp. 1-9

[20] "Ball Grid Arrays in Printed Circuit Board Assembly." Internet: http://www.thomasnet.com/articles/custom-manufacturing-fabricating/pcb-ballgrid-arrays 
[21] V. Gektin and A. Bar-Cohen. "Coffin-Manson Fatigue Model of Underfilled Flip-Chips," in Proc. IEEE Transactions on Components, Packaging and Manufacturing Technology, Sep. 1997, pp. 317-326

[22] P. Chauhan, M. Osterman, S.W.R. Lee and M. Pecht. "Critical Review of Engelmaier Model for Solder Joint Creep Fatigue Reliability,” in Proc. IEEE Transactions on Components and Packaging Technology, Sep. 2009, pp. 693700

[23] W. Engelmaier. “Creep-Fatigue Model for SAC405/305 Solder Joint Reliability Estimation-A Proposal," in Proc. Global SMT and Packaging, 2008

[24] "Cauchy Stress Tensor." Internet: http://en.wikipedia.org/wiki/Cauchy_stress_tensor

[25] "Von-Mises Yield Criterion" Internet: http://en.wikipedia.org/wiki/Von_Mises_yield_criterion

[26] Sanpaz. "Components of Cauchy Stress Tensor.” Internet: http://commons.wikimedia.org/wiki/File:Components_stress_tensor_cartesian.sv $\mathrm{g}$

[27] Rswarbick. "Yield Surfaces." Internet: http://commons.wikimedia.org/wiki/File:Yield_surfaces.svg

[28] J.P. Calame, R.E. Myers, F.N. Wood and S.C. Binari. "Simulations of DirectDie-Attached Microchannel Coolers for the Thermal Management of GaN-onSiC Microwave Amplifiers," in Proc. IEEE Transactions on Components and Packaging Technologies, Dec. 2005, pp. 797-809

[29] M.L. Minges. Electronics Materials Handbook: Packaging. ASM International, Nov. 1989

[30] H. Ma, J.C. Suhling, P. Lall and M. J. Bozack. "Reliability of the Aging Lead Free Solder Joint," in Proc. Electronic Components and Technology Conference, 2006, pp. 849-864

[31] Rolf Sandstrom and Josefin Hallgren. "Stress Strain Flow Curves for Cu-OFP," Royal Institute of Technology (KTH) Internet: http://www.iaea.org/inis/collection/NCLCollectionStore/_Public/40/065/400653 03.pdf [Jan. 2014]

[32] S.H. Choday, M.S. Lundstrom and K. Roy. "Prospects of Thin-Film Thermoelectric Devices for Hot-spot Cooling and On-chip Energy Harvesting," in Proc. IEEE Transactions on Components, Packaging and Manufacturing Technology, Dec. 2013, pp. 2059-2067

[33] N.E. Dowling, "Mechanical Behavior of Materials." Prentice Hall, 1993

[34] A. Dasgupta. Class Lecture, Topic: "Modeling Material Behavior." Department of Mechanical Engineering, University of Maryland at College Park, MD, Spring 2013

[35] P. Wang, B. Yang and A. Bar-Cohen. (2009). "Mini-contact Enhanced Thermoelectric Coolers for on-chip Hot-spot Cooling." Heat Transfer Engineering, Taylor and Francis. Vol. 30. Pp. 736-743. Available: http://www.tandfonline.com/doi/full/10.1080/01457630802678391\#tabModule [Jan 20, 2014]

[36] B. Yang, P. Wang and A. Bar-Cohen. "Thermoelectric Mini-contact Cooler for Hot-spot Removal in High Power Devices," in Proc. ECTC, 2006, pp. 997 - 1002 
[37] B. Yang, P. Wang and A. Bar-Cohen. "Mini-Contact Enhanced Thermoelectric Cooling of Hot Spots in High Power devices," in Proc. IEEE Transactions on Components and Packaging Technologies, 2007, Vol. 30, pp. 432-438.

[38] M. Ohadi, K. Choo, S. Dessiatoun and E. Cetegen. Next Generation Microchannel Heat Exchangers. Springer New York Heidelberg Dordrecht London, 2013, pp. 33-64.

[39] J.G. Bai, Z.Z. Zhang, J.N. Calata and G.Q. Lu, "Low-Temperature Sintered Nanoscale Silver as a Novel Semiconductor Device-Metallized Substrate Interconnect Material," in Proc. IEEE Transactions on Components and Packaging Technologies, Vol. 29, No. 3, Sep. 2006

[40] D.J. Yu, X. Chen, G. Chen, G. Lu and Z. Wang. "Applying Anand Model to Low-Temperature Sintered Nanoscale Silver Paste Chip Attachment," Journal of Materials and Design, No. 30, Dec. 2009

[41] "CVD Diamond for Thermal Applications" Internet: http://www.e6.com/wps/wcm/connect/272cb7b5-aa22-4c95-aedb8977925338e7/DiafilmTM+Datasheet_USSIZE_r2_v2.pdf?MOD=AJPERES\&C ACHEID=272cb7b5-aa22-4c95-aedb-8977925338e7

[42] "Microwave CVD Diamond for Superior Thermal Management Solutions." Internet: http://www.e6.com/wps/wcm/connect/fe8800c0-ed2c-4ceb-99b6fe16928b0664/DIAFILM+TM_030613.pdf?MOD=AJPERES\&CACHEID=fe88 00c0-ed2c-4ceb-99b6-fe16928b0664

[43] A. Abyzov, S.V. Kidalov and F.M. Shakhov. "High Thermal Conductivity Composite of Diamond Particles with Tungsten Coating in a Copper Matrix for Heat Sink Application.” Applied Thermal Engineering, vol. 48, pp. 72-80, 2012

[44] E. Sideridis and G.C. Papanicolau. "A Theoretical Model for the Prediction of Thermal Expansion Behavior of Particulate Composites." Rheologica Acta, vol. 27, pp. $608-616,1988$

[45] C. Zweben. "Metal-matrix Composites for Electronic Packaging." Journal of Materials, Vol. 44, Issue 7, pp. 15-23, Jul.1992

[46] "Thin Film Thermoelectric Module - HV37." Internet: http://www.lairdtech.com/brandworld/library/THR-DS-eTECHV37\%201113.pdf

[47] "Material Properties." Internet: http://www.matweb.com/

[48] "Material Properties." Internet: https://www.wikipedia.org/

[49] M. Pecht, R. Agarwal, F.P. McCluskey, T.J. Dishongh, S. Javadpour and R. Mahajan. Electronic Packaging Materials and Their Properties. CRC Press, Technology and Engineering, Dec. 18, 1998

[50] "Intrachip/Interchip Enhanced Cooling Fundamentals (ICECool Fundamentals)." Internet: https://www.fbo.gov/?s=opportunity\&mode=form\&id=d8db5ea850b52ec3a5ebc $2649 \mathrm{e} 11 \mathrm{f} 798 \& \mathrm{tab}=\mathrm{core} \& \_\mathrm{cview}=1$

[51] "Aluminum Nitride, AlN Ceramic Properties." Internet: http://accuratus.com/alumni.html

[52] I. Yonenaga. "Thermo-mechanical stability of wide-bandgap semiconductors: high temperature hardness of $\mathrm{SiC}, \mathrm{AlN}, \mathrm{GaN}, \mathrm{ZnO}$ and $\mathrm{ZnSe}$," in Proc. 
International Conference on Defects in Semiconductors, Vol. 30, Dec. 2001, pp. $1150-1152$

[53] "Stress Concentration." Internet:

http://www.ux.uis.no/ hirpa/KdB/ME/stressconc.pdf

[54] "Weibull Distribution, copy right information." Internet:

http://reliawiki.org/index.php/Copyright_Information

[55] "ANSYS Mechanical." Internet:

http://www.ansys.com/Products/Simulation+Technology/Structural+Analysis/A

NSYS+Mechanical 